\title{
Ribosomal Asc1p/RACK1 \\ in the phosphorylation signaling network of Saccharomyces cerevisiae
}

\section{Dissertation}

\author{
for the award of the degree \\ "Doctor rerum naturalium" \\ of the Georg-August-Universität Göttingen
}

within the doctoral program "Molecular Biology of Cells"

of the Georg-August University School of Science (GAUSS)

submitted by

\section{Kerstin Schmitt}

from Göttingen 


\section{Thesis Committee}

Dr. Oliver Valerius

Department of Molecular Microbiology and Genetics

Institute of Microbiology and Genetics, Georg-August-University Göttingen

Prof. Dr. Heike Krebber

Department of Molecular Genetics

Institute of Microbiology and Genetics, Georg-August-University Göttingen

Prof. Dr. Rolf Daniel

Department of Genomic and Applied Microbiology

Institute of Microbiology and Genetics, Georg-August-University Göttingen

\section{Members of the Examination Board}

Referee: Dr. Oliver Valerius

Department of Molecular Microbiology and Genetics

Institute of Microbiology and Genetics, Georg-August-University Göttingen

$2^{\text {nd }}$ Referee: Prof. Dr. Heike Krebber

Department of Molecular Genetics

Institute of Microbiology and Genetics, Georg-August-University Göttingen

\section{Further members of the Examination Board}

Prof. Dr. Gerhard H. Braus

Department of Molecular Microbiology and Genetics

Institute of Microbiology and Genetics, Georg-August-University Göttingen

Prof. Dr. Rolf Daniel

Department of Genomic and Applied Microbiology

Institute of Microbiology and Genetics, Georg-August-University Göttingen

Prof. Dr. Kai Heimel

Department of Molecular Microbiology and Genetics

Institute of Microbiology and Genetics, Georg-August-University Göttingen

Prof. Dr. Stefanie Pöggeler

Department of Genetics of Eukaryotic Microorganisms

Institute of Microbiology and Genetics, Georg-August-University Göttingen

Date of oral examination: $17^{\text {th }}$ February 2016 
I hereby declare that the doctoral thesis entitled "Ribosomal Asc1p/RACK1 in the phosphorylation signaling network of Saccharomyces cerevisiae" has been written independently and with no other sources and aids than quoted.

Kerstin Schmitt 
Parts of this work are published in:

Rachfall, N., Schmitt, K., Bandau, S., Smolinski, N., Ehrenreich, A., Valerius, O., and Braus, G.H. (2013). RACK1/Asc1p, a ribosomal node in cellular signaling. Mol Cell Proteomics 12, 87-105.

Schmitt, K., Smolinski, N., Neumann, P., Schmaul, S., Hofer-Pretz, V., Braus, G.H., and Valerius, O. (2017). Asc1p/RACK1 Connects Ribosomes to Eukaryotic Phosphosignaling. Mol Cell Biol 37, e00279-16

This work was supported by the Göttingen Graduate School for Neurosciences and Molecular Biosciences (DFG Grant GSC 226/2) and by the DFG Grant VA352/2-1. 


\section{Acknowledgements - Danksagung}

Ich möchte mich an erster Stelle bei Dr. Oliver Valerius für die hervorragende und engagierte Betreuung dieser Arbeit bedanken: Vielen Dank für die zahlreichen gemeinsamen Gespräche und Diskussionen, das entgegengebrachte Vertrauen und die Begeisterung für das Projekt.

Prof. Dr. Gerhard H. Braus danke ich für die Möglichkeit meine Arbeit in der Abteilung Molekulare Mikrobiologie und Genetik anfertigen zu können und für die damit verbundene Unterstützung sowie das Interesse an meiner Arbeit.

Prof. Dr. Heike Krebber und Prof. Dr. Rolf Daniel danke ich für die hilfreichen Diskussionen im Rahmen der Thesis Committee Meetings und darüber hinaus für die Kooperationen mit ihren Abteilungen. Aus der Arbeitsgruppe Molekulare Genetik danke ich Dr. Bettina Neumann für die Einführung in die Methode der Dichtegradientenzentrifugation und für viele wichtige Hinweise und Ratschläge. Dr. Andrea Thürmer, Kathleen Gollnow und Dr. Sascha Dietrich aus der Abteilung Genomische und Angewandte Mikrobiologie bin ich sehr dankbar für die gemeinsame Versuchsplanung, die Durchführung der cDNA Synthese und Sequenzierung und für die anschließende gemeinsame Datenanalyse.

Ein großer Dank gilt allen ehemaligen und aktuellen Mitgliedern des Labors, insbesondere Dr. Britta Herzog, Anika Kühn, Dr. Harald Kusch, Katharina Mucek, Verena Hofer-Pretz, Dr. Nicole Rachfall, Sabrina Sander, Samantha Schmaul, und Nadine Smolinski für die großartige Unterstützung und die schöne Zeit auch außerhalb des Labors.

Ein besonders großer und herzlicher Dank gilt Verena Hofer-Pretz für ihre ausgezeichnete und verlässliche Arbeit. Nadine Smolinski, Sabrina Sander, Samantha Schmaul und Katharina Mucek haben durch ihre Praktika und Abschlussarbeiten einen wesentlichen Beitrag zu dieser Arbeit geleistet, für den ich sehr dankbar bin. Nadine Smolinski danke ich für die enge Zusammenarbeit an dem Projekt auch über ihre Masterarbeit hinaus. Besonders möchte ich mich auch bei Anika Kühn bedanken, die vor allem bei methodischen Fragen immer eine ausgezeichnete Ansprechpartnerin war.

Der gesamten Abteilung Molekulare Mikrobiologie und Genetik danke ich für die angenehme Arbeitsatmosphäre und hilfreiche Diskussionen. Der Göttinger Graduiertenschule für Neurowissenschaften, Biophysik und Molekulare Biowissenschaften danke ich für die umfassende finanzielle Unterstützung dieser Arbeit.

Meiner Familie und insbesondere meinen Eltern danke ich für ihre Unterstützung und das Interesse an meiner Arbeit. 


\section{Table of Contents}

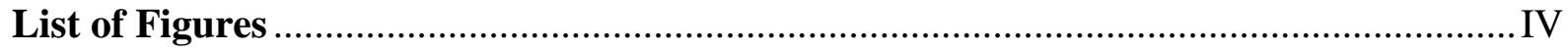

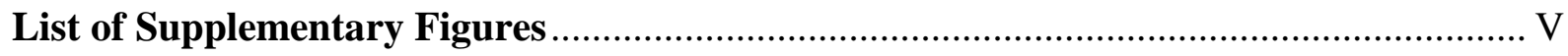

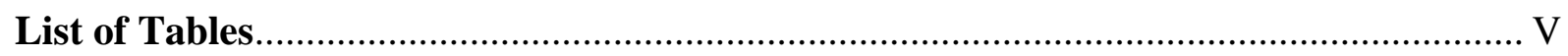

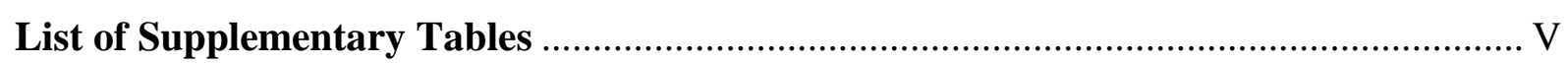

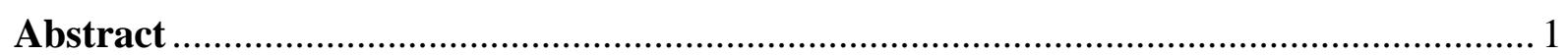

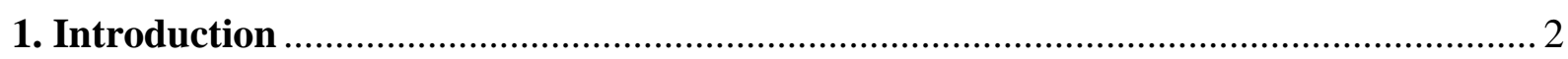

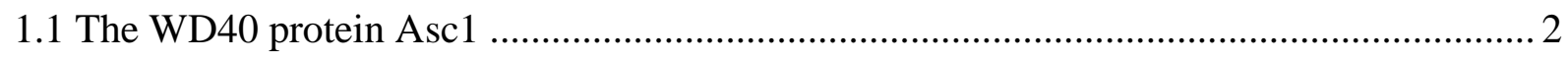

1.2 The G $\beta$-like protein Asc1 - an integral component of the ribosome.................................. 4

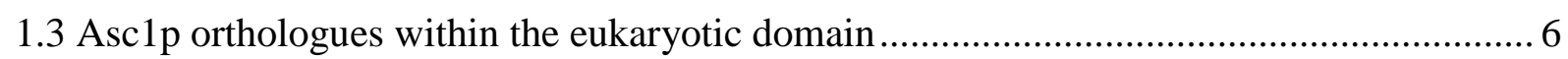

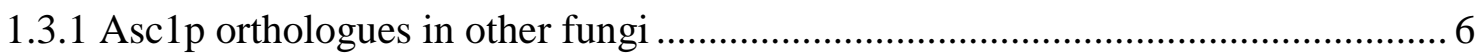

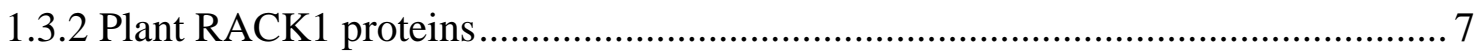

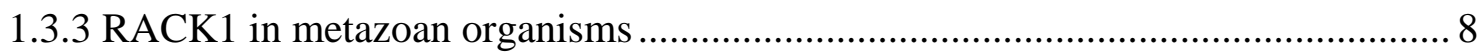

1.3.4 ASC1/RACK1 gene expression correlates with ribosomal genes .......................... 9

1.3.5 Orthologous genes complement ASC1/RACK1-deficiency across phyla................ 9

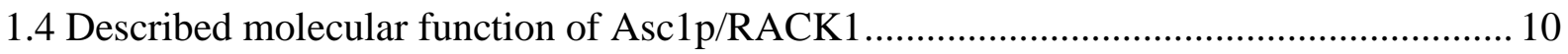

1.4.1 Asc1p/RACK1 as a regulator of mRNA translation ......................................... 10

1.4.1.1 S. cerevisiae Asc1p in co-translational quality control and reading frame

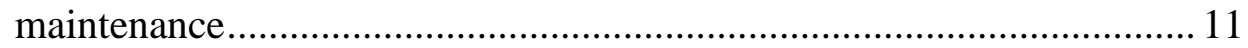

1.4.1.2 Asc1p/RACK1-dependent phosphorylation of translation factors ........... 12

1.4.1.3 Asc1p/RACK1-dependent recruitment of proteins to the ribosome......... 13

1.4.1.4 RACK1's function in IRES-dependent translation initiation and the

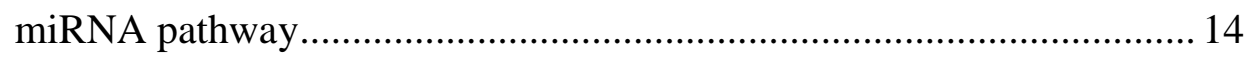

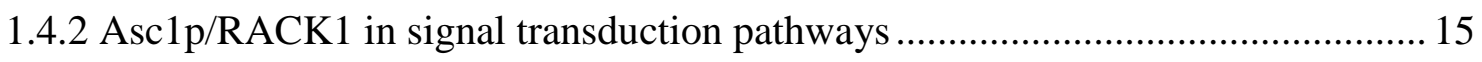

1.4.2.1 Role of Asc1p and its orthologues in signal transduction pathways in fungi

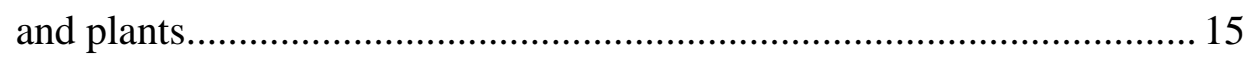

1.4.2.2 Metazoan RACK1 - Receptor for activated C kinase 1 ........................... 17

1.4.2.3 RACK1 links insulin-like growth factor 1 with integrin signaling........... 18

1.4.2.4 Metazoan RACK1 in cAMP/PKA signaling ......................................... 19

1.4.2.5 Function of RACK1 in MAPK cascades .............................................. 21

1.4.2.6 RACK1 as a central hub linking signal transduction with translation...... 24

1.4.3 RACK1-dependent protein-degradation of its interaction partners ...................... 24

1.5 Regulation of Asc1p/RACK1 through post-translational modifications .......................... 26

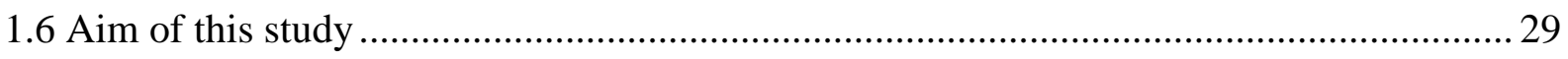




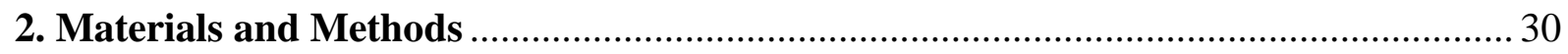

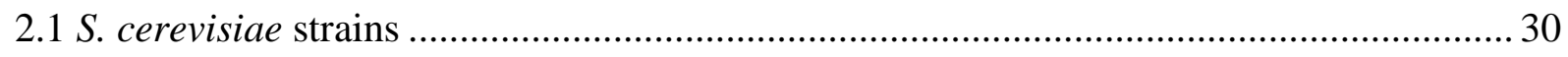

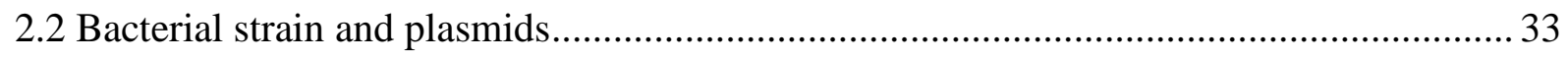

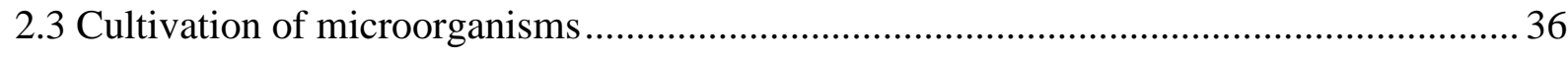

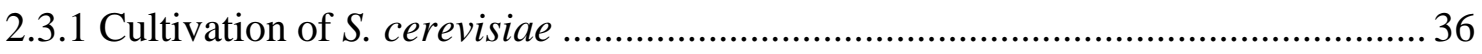

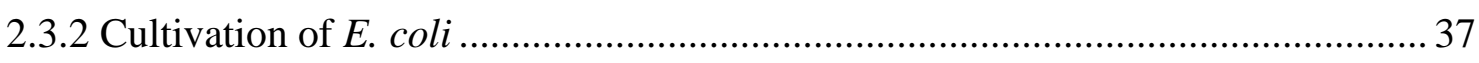

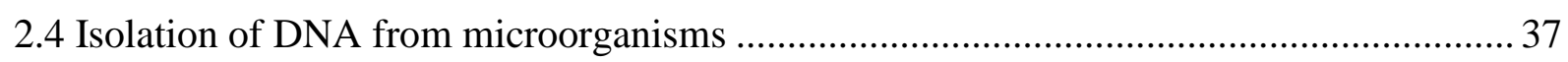

2.4.1 Plasmid DNA purification from E. coli .......................................................... 37

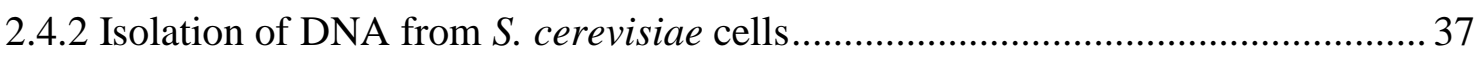

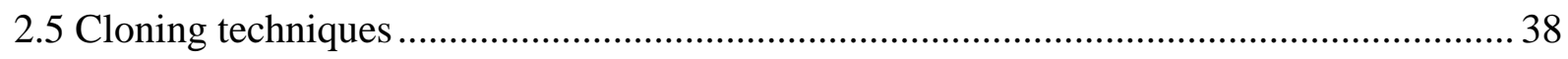

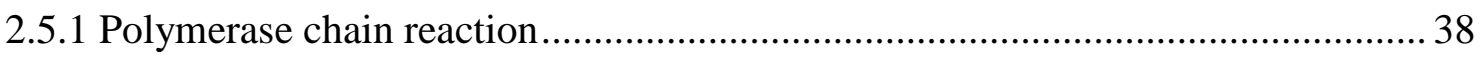

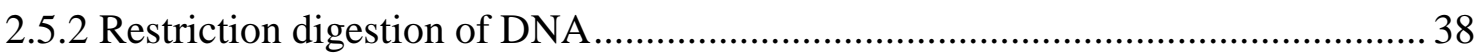

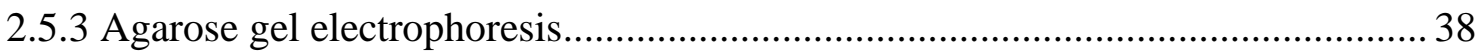

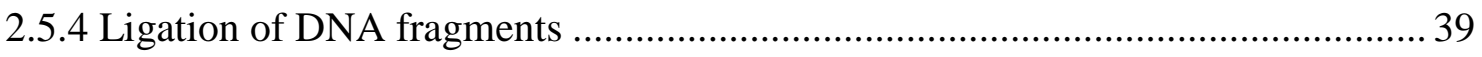

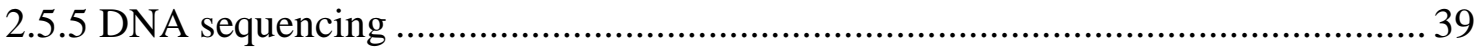

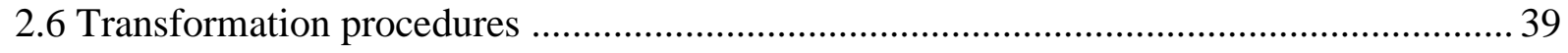

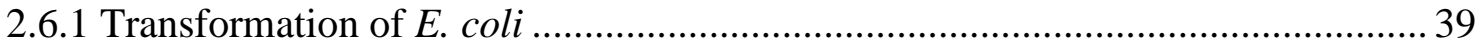

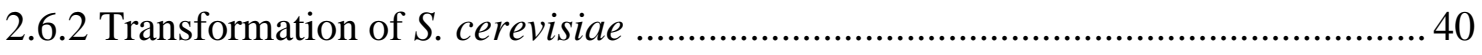

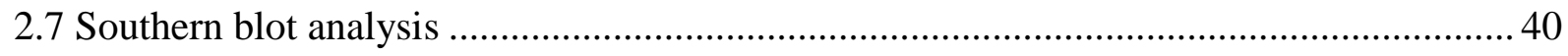

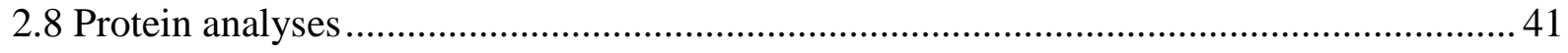

2.8.1 Preparation of whole-cell protein extracts from $S$. cerevisiae ............................. 41

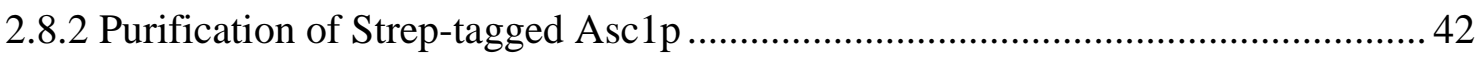

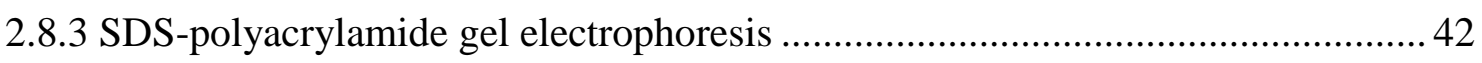

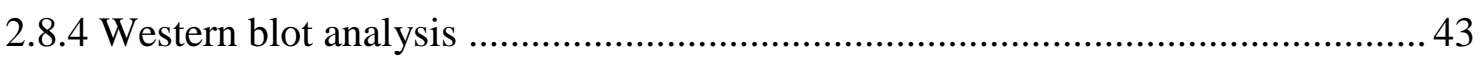

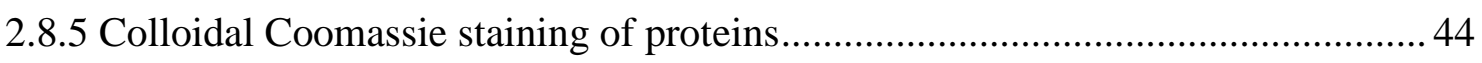

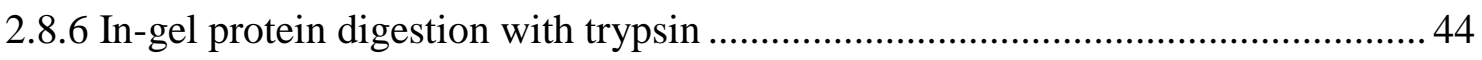

2.8.7 In-solution digestion of proteins with LysC and trypsin..................................... 46

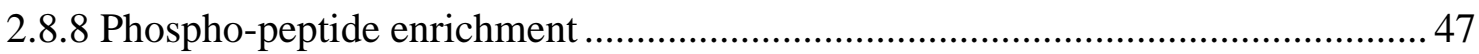

2.8.9 Liquid chromatography-mass spectrometry analysis ...................................... 48

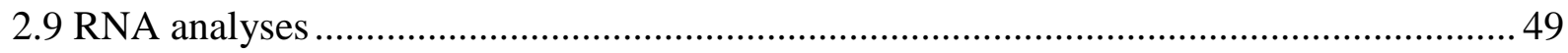

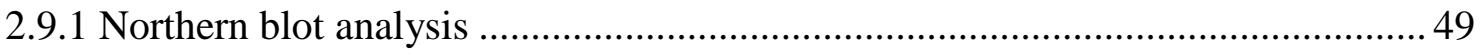

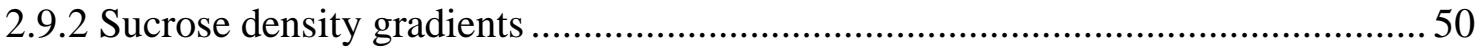

2.9.3 Isolation of RNA from sucrose gradient fractions and mRNA enrichment ...........50

2.9 .4 cDNA synthesis and RNASeq analysis....................................................... 51

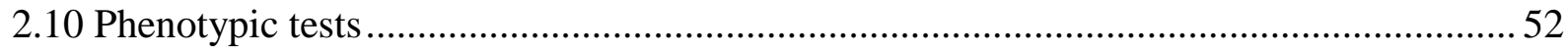




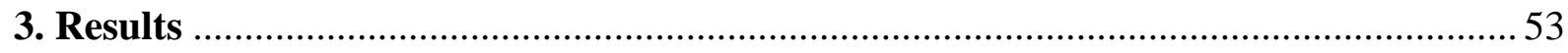

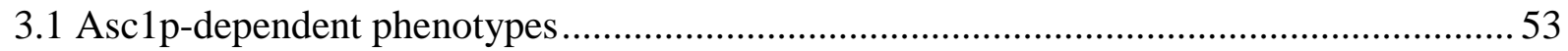

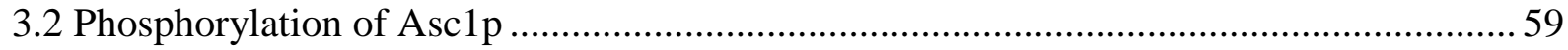

3.2.1 The surface of Asc1p is target for multiple phosphorylations ............................. 60

3.2.2 Construction of yeast strains expressing Asc1p isoforms with amino acid

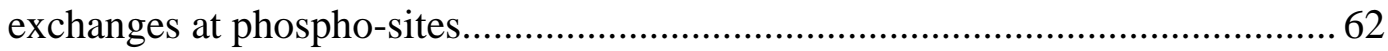

3.2.3 Asc1p phospho-sites T143 and Y250 confer resistance against translation inhibitors and together with T12, T96, and T99 are essential to maintain overall functionality of the Asc1DEp variant.

3.2.4 Phosphorylation of T12, T96, T99, and especially T143 is required for cellular abundance of Asc1DEp 68

3.3 The Asc1p-dependent phospho-proteome 69

3.3.1 Asc1p affects the phosphorylation of more than 200 proteins at almost 300 different sites .70

3.3.2 Asc1p spreads signals to fundamental processes of eukaryotic gene expression 74

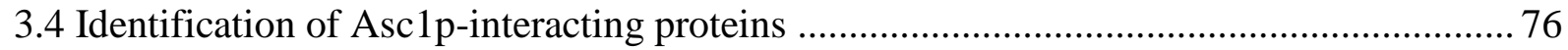

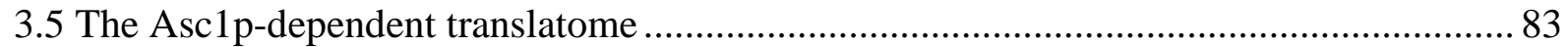

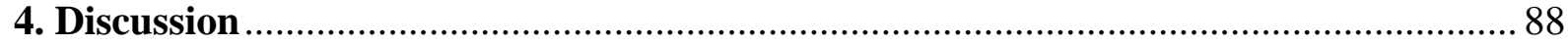

4.1 Does the R38D K40E amino acid exchange within Asc1p cause a ribosome binding defect in vivo? 88

4.2 The DE exchange and phospho-site mutations synergistically compromise Asclp's

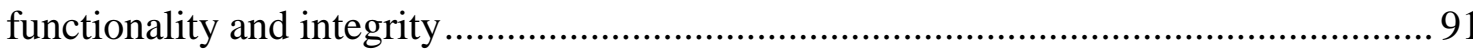

4.3 Absence of Asc1p significantly changes the phospho-proteome of $S$. cerevisiae ............. 95

4.4 Versatile Asc1p-dependent changes in the phospho-proteome: How does Asc1p act?..... 96

4.5 Asc1p affects localized mRNA translation and cytoskeleton organization ...................... 99

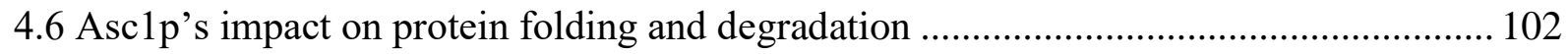

4.7 The $\beta$-propeller Asc1p represents a regulatory interface connecting mRNA translation with fundamental cellular processes according to signals .......................................... 105

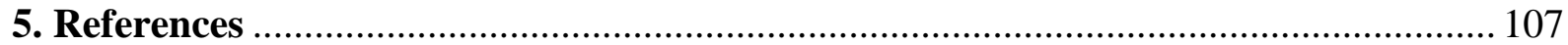

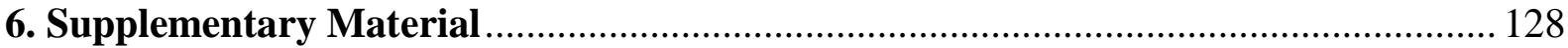

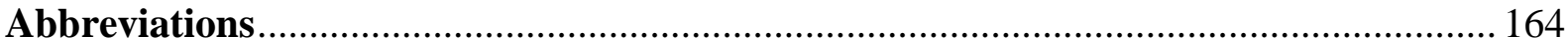




\section{List of Figures}

Figure 1. Structural features of the WD40 protein Asc1 ...................................................... 3

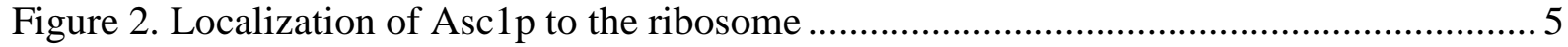

Figure 3. Model on Asc1p's function in the cAMP/PKA pathway and MAPK cascades in S. cerevisiae

Figure 4. Model on the function of RACK1 in IGF-1R signal transduction

Figure 5. Model on the involvement of RACK1 in G-protein and cAMP/PKA signaling in

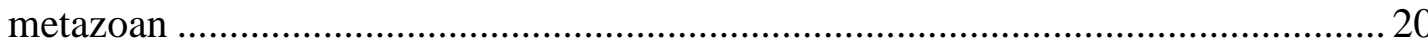

Figure 6. Model on the involvement of RACK1 in MAPK cascades in metazoans ................ 22

Figure 7. Model on RACK1-dependent and oxygen-independent regulation of HIF-1 $\alpha$ stability 25

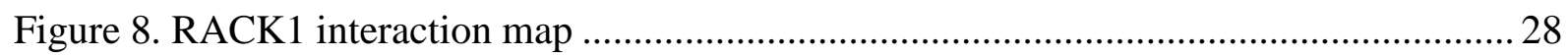

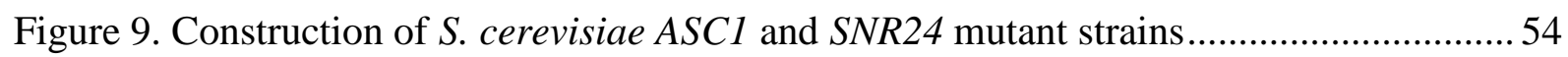

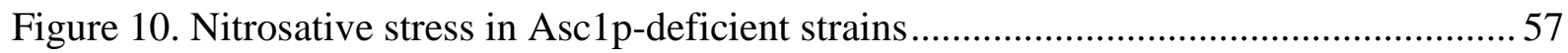

Figure 11. Phenotypic characterization of the asclSNR24, cASC1, and asc1DE strains......... 58

Figure 12. Asc1p-dependent transcription factor levels......................................................5 59

Figure 13. LC-MS-based identification of Asc1p phospho-sites ........................................... 60

Figure 14. Cartoon view of Asc1p bound to the 40S subunit of the ribosome

Figure 15. Drop dilution assays to analyze the impact of phospho-site mutations on Asc1p dependent phenotypes

Figure 16. Impact of Asc1p phospho-site mutations on colony morphology and respiratory activity..... 65

Figure 17. Test for haploid adhesive growth of phospho-site mutant strains 66

Figure 18. Complementation of ASC1 phospho-site mutant phenotypes by expression of plasmid-borne wild-type $A S C 1$ 67

Figure 19. Asc1 protein abundance in phospho-site mutant strains......................................6 68

Figure 20. Asc1p-dependency of the high osmolarity response pathway ..............................69

Figure 21. Analysis of the Asc1p-dependent phospho-proteome ......................................... 71

Figure 22. Cellular processes and known Asc1p-interaction partners that are targeted by Asc1p-dependent phosphorylation. 75

Figure 23. Workflow for the identification of putative interaction partners of Asc1p-Strep and Asc1DEp-Strep 77

Figure 24. Interaction map of Asc1p-Strep and Asc1DEp-Strep ........................................ 82

Figure 25. Workflow for the Asc1p-dependent translatome and transcriptome analysis 


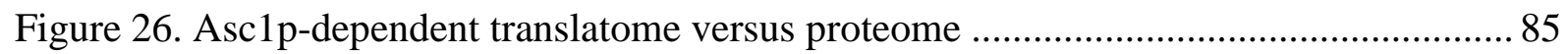

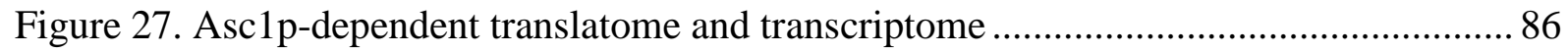

Figure 28. Phospho-site locations within Asc1p and its orthologues .................................... 92

Figure 29. Phosphorylation in the blade 3 region of Asc1p/RACK1 ................................... 94

Figure 30. Overrepresented motifs for Asc1p-sensitive phosphorylated serine and threonine

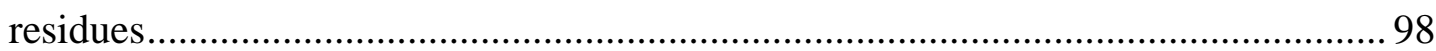

Figure 31. Asc1p-dependent alterations of the 26S proteasome ......................................... 103

Figure 32. The rack-wheel of Asc1p-dependent signal transduction ................................... 106

\section{List of Supplementary Figures}

Supplementary Figure 1. DNA and amino acid sequence alignments of the ASC1 SNR24 wildtype strain and the asc $^{-}$strain 128

Supplementary Figure 2. Annotated fragmentation spectra of Asc1p phospho-peptides ...... 130

\section{List of Tables}

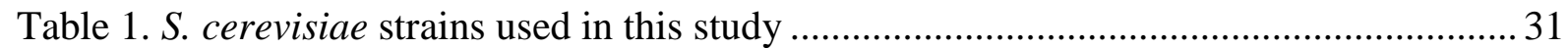

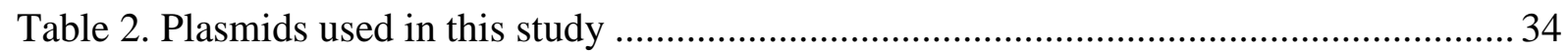

Table 3. Overview of Asc1p phospho-site mutant strains and their phenotypes .....................63

Table 4. Asc1p-dependently regulated phospho-sites ......................................................... 72

Table 5. Logarithmized SILAC-ratios of proteins co-captured with Asc1p-Strep .................. 78

Table 6. Logarithmized SILAC-ratios of proteins co-captured with Asc1DEp-Strep ............. 79

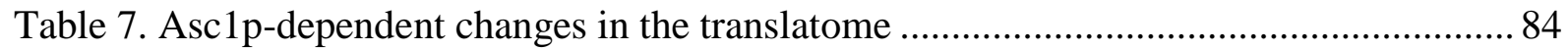

\section{List of Supplementary Tables}

Supplementary Table 1. LC-MS-based identification of Asc1p-derived phospho-peptides 129

Supplementary Table 2. Overview of proteome data evaluation with Perseus .................... 139

Supplementary Table 3. Overview of phospho-proteome data evaluation with Perseus ...... 140

Supplementary Table 4. SILAC-based proteome data for the comparison of the sscl $^{-}$strain and the $A S C 1$ wild-type strain

Supplementary Table 5. SILAC-based proteome data for the comparison of the asc1DE strain and the $A S C 1$ wild-type strain

Supplementary Table 6. SILAC-based proteome data for the comparison of the asc $1^{\text {T143A }}$

strain and the $A S C l$ wild-type strain 146 
Supplementary Table 7. SILAC-based proteome data for the comparison of the ascl ${ }^{\mathrm{T} 143 \mathrm{E}}$ strain and the $A S C 1$ wild-type strain 146

Supplementary Table 8. SILAC-based proteome data $a s c 1^{\text {T143E }}$ DE versus ASC1 wild-type 147

Supplementary Table 9. SILAC-based phospho-proteome data for the comparison of the ascl- $^{-}$ strain and the $A S C 1$ wild-type strain

Supplementary Table 10. SILAC-based phospho-proteome data for the comparison of the asc $1^{\mathrm{T} 143 \mathrm{~A}}$ strain and the $A S C 1$ wild-type strain

Supplementary Table 11. SILAC-based phospho-proteome data for the comparison of the asc $1^{\text {T143E }}$ strain and the $A S C 1$ wild-type strain

Supplementary Table 12. Cellular processes affected by Asc1p-dependent phosphorylation

Supplementary Table 13. Overview of data evaluation of SILAC-based Asc1p-Strep and Asc1DEp-Strep enrichment experiments with Perseus

Supplementary Table 14. Expanded view for the Asc1p-dependent changes in the translatome 158

Supplementary Table 15. Mitochondrial proteins down-regulated in their abundance in the ascl $^{-}$strain. 160

Supplementary Table 16. Proteasomal proteins up-regulated in their abundance in the asc $1^{-}$ strain 


\section{Abstract}

The WD40 scaffold protein Asc1 of Saccharomyces cerevisiae solely consists of a sevenbladed $\beta$-propeller. The protein is highly conserved among eukaryotes and known as RACK1 in metazoans and plants. Asc1p/RACK1 localizes to the head of the 40S ribosomal subunit next to the mRNA exit channel and associates with proteins of signal transduction pathways, thus providing a link between cellular signaling and mRNA translation. In this work, phosphorylation of Asc1p was analyzed by mass spectrometry resulting in the identification of at least three previously unknown phosphorylation sites. To analyze the impact of phosphorylation on the functionality of the protein, Asc1p phospho-site mutant strains were generated for these residues as well as for five additional phospho-sites known from highthroughput studies. Phenotypic characterization showed slightly increased sensitivity to translation inhibitors caused by dephospho-mimics at T143 and Y250, whereas all other phospho-site mutations caused no obvious effect. However, phosphorylation of T12, T96, T99, and especially T143 is required to maintain the abundance of the ribosome bindingcompromised Asc1DEp variant. According to the reduced level of the dephospho-mimicking DE-variants, the respective mutant strains show ascl- phenotypes. Mutation of Y250 did not change Asc1DEp abundance, but also resulted in $a s c 1^{-}$phenotypes with respect to cycloheximide sensitivity. Asc1p has a strong impact on the cellular phospho-proteome. A SILAC-based LC-MS approach identified almost 300 Asc1p-sensitive phosphorylation sites in more than 200 proteins that are mainly involved in translation and signal transduction but also other processes. Identification of Asc1p-associated proteins, including, for instance, mRNA-binding proteins, further corroborate Asc1p's involvement in some of these processes, such as mRNA translation. With RNASeq analysis of polysome-derived mRNAs the translatomes of wild-type ASCl and $\mathrm{ascl}^{-}$strains were compared. Among 73 transcripts of differentially translated mRNAs, the $Y H R 177 \mathrm{~W}$ mRNA is very remarkable as the total level of the transcript significantly increases, whereas its polysome association decreases. Yhr177wp belongs to the fungal-specific family of WOPR transcription factors that regulate morphology and development. Altogether, this work verifies the conserved $\beta$-propeller Asc1p as an important rack-wheel within cellular adaptation, signaling, and process coordination. 


\section{Introduction}

\subsection{The WD40 protein Asc1}

Asc1p from $S$. cerevisiae is a highly conserved eukaryotic protein and belongs to the family of WD40 proteins (Chantrel et al., 1998). The WD40 domain belongs to the most abundant domain types in eukaryotes, while there are only few examples known for prokaryotic WD40 proteins (Stirnimann et al., 2010). The WD40 domains provide scaffolds for the formation of protein complexes and harbor no intrinsic catalytic function known so far. In contrast to the Asc1 protein, which solely consists of a single WD40 domain, many members of this protein family contain additional catalytic or functional domains (Stirnimann et al., 2010). They function in various processes, such as signal transduction, RNA synthesis, vesicular trafficking, cytoskeleton assembly, cell division, and apoptosis (Li and Roberts, 2001).

A WD40 domain comprises four to sixteen repeats of 44 to 66 amino acids with low sequence conservation. Each repeat typically contains a glycine-histidine dipeptide in its $\mathrm{N}$-terminal region and ends with a tryptophan-aspartate (WD) motif (Figure 1A, Li and Roberts, 2001). The domain folds into a $\beta$-propeller with each blade consisting of a four-stranded anti-parallel $\beta$-sheet. The four $\beta$-strands of each sheet are referred to as A, B, C, and D starting with A from the center of the propeller (Figure 1B). Each WD40 repeat is part of two blades providing strand $\mathrm{D}$ of one $\beta$-sheet and strands $\mathrm{A}, \mathrm{B}$, and $\mathrm{C}$ of the following sheet. The narrower side of the propeller with the loops connecting strands D and A as well as B and C is defined as the top side (Figure 1C, Li and Roberts, 2001). Together with the bottom side and the circumference of the propeller the structure provides a large surface for protein-protein, protein-peptide, and protein-nucleic acid interactions (Stirnimann et al., 2010). The Asc1 protein has the most common $\beta$-propeller structure with seven blades, which seems to have the optimal $\beta$-propeller-fold (Figure 1C, Murzin, 1992; Coyle et al., 2009). In S. cerevisiae, high-throughput interaction studies showed that the WD40 domain has a higher number of interactions than any other domain in yeast-two hybrid assays, which identify binary interactions, as well as in mass spectrometry/tandem affinity purification experiments, which include interactions within protein complexes (Stirnimann et al., 2010). The number of interactions found in the latter experiments tremendously exceeds the number found in the yeast-two hybrid assays implicating the participation of WD40 proteins in formation of large protein complexes (Stirnimann et al., 2010).

WD40 proteins can also interact with each other as it was, for instance, described that the mammalian orthologue of Asc1p, RACK1, interacts with different G $\beta$ isoforms (Chen et al., 2004b) and forms homodimers (Thornton et al., 2004; Liu et al., 2007b). So far, there is no 


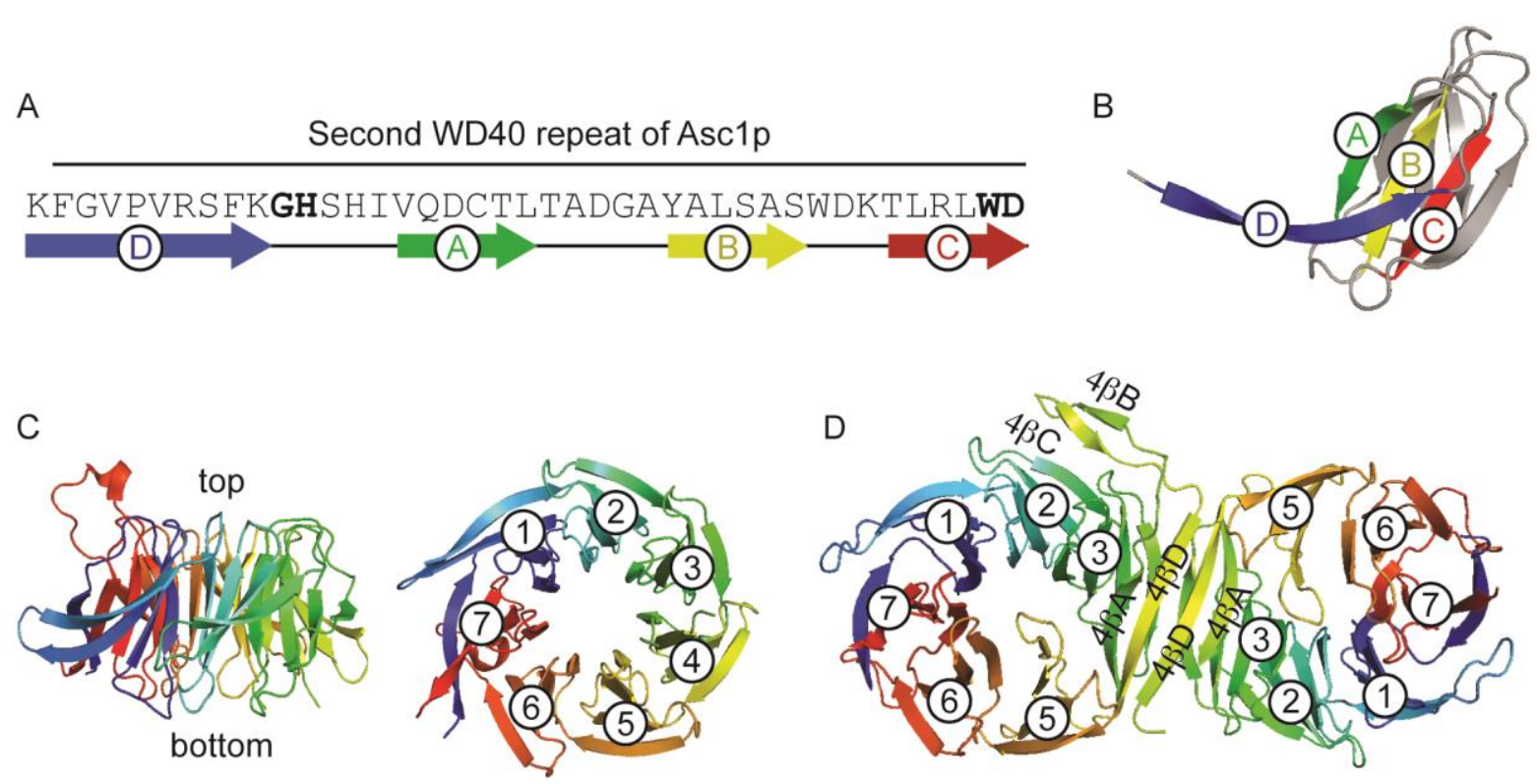

Figure 1. Structural features of the WD40 protein Asc1.

(A) Amino acid sequence of the second WD40 repeat of Asc1p with the characteristic GH and WD motifs printed in bold. The arrows below the sequence labeled D, A, B, and C indicate the amino acids that form $\beta$-strands. (B) The structure formed by the second WD40 repeat of Asc1p. $\beta$-strands are colored according to the color-code used in A illustrating that each WD40 repeat is in general part of two blades. (C) Side- and bottom-view on the seven-bladed Asc1p colored with a gradient from blue (N-terminus) to red (C-terminus). Blades are labeled from 1 to 7 starting at the $\mathrm{N}$-terminus. (D) Asc1p homodimer. Both Asc1 proteins are depicted with the same coloring as in C. Blades are labeled from 1 to 7 . The individual $\beta$-strands of blade 4 are labeled from $4 \beta A$ to $4 \beta D$. Strands $4 \beta B$ and $4 \beta C$ of Asc1p depicted on the right-hand side were not resolved in the crystal structure and are therefore not present in the depicted structure. All structures were generated with the PyMOL Molecular Graphics System software based on the Protein Data Bank (PDB) files 3FRX and 3RFH (Coyle et al., 2009; Yatime et al., 2011). The figure is adapted from Stirnimann et al. (2010).

experimental evidence that S. cerevisiae Asc1p forms homodimers in vivo, however, a crystal structure of a homodimer derived from recombinantly expressed Asc1p has been resolved (Figure 1D, Yatime et al., 2011). In contrast to other $\beta$-propeller dimers, the Asc1p homodimer shows an atypical structural rearrangement. While the overall shape of each Asc1p molecule is preserved in the dimer, the inner strands $\mathrm{B}$ and $\mathrm{C}$ of both blades number four are expelled from the propeller, and the outer strands A and D form a new blade that is shared by both molecules (Figure 1D, Yatime et al., 2011). In the dimer, the planes of the two Asc1 proteins are oriented in an almost $90^{\circ}$ angle to each other. The homodimer does not only provide an extended surface for interactions, it also exposes regions of the protein that are otherwise not accessible in the monomeric form (Yatime et al., 2011). Homodimerization enables Asc1p/RACK1 to scaffold the interaction between two proteins that share the same binding site of the protein as it was shown for the mammalian NMDA receptor and the Fyn kinase that both bind to the first $\beta$-propeller blade of RACK1 (Thornton et al., 2004). Homodimerization of mammalian RACK1 also seems to be essential for the interaction 
between the transcription factor subunit HIF-1 $\alpha$ and the Elongin-C E3 ubiquitin ligase complex that both interact with the sixth WD40 repeat of RACK1 (Liu et al., 2007b). Results from the two studies hint to the same region of RACK1 for dimerization as it is observed for the crystal structure of the Asc1p homodimer.

\subsection{The G $\beta$-like protein Asc1 - an integral component of the ribosome}

There is only one WD40 repeat protein known in S. cerevisiae that is almost exclusively composed of a single $\beta$-propeller like Asclp, namely the G $\beta$-protein Ste4 of the pheromone response pathway. Asc1p might act in an analogous way to Ste4p. The Ste4p-comprising heterotrimeric G-protein associates to the transmembrane receptor Ste2p of MATa or Ste3p of MAT $\alpha$ cells, respectively. Binding of pheromones ( $\alpha$ - or a-factor, respectively) to the receptors triggers their conformational change leading to guanosine diphosphate (GDP) to guanosine triphosphate (GTP) exchange at the Ga-subunit Gpa1p. The reduced affinity of the GTP-bound Gpalp for the G $\beta \gamma$-heterodimer (Ste4p and Ste18p) leads to its release from the complex. G $\beta \gamma$ subsequently activates downstream effectors that finally regulate gene expression for the induction of the mating process (pheromone signal transduction in yeast is reviewed in Bardwell, 2005). Asc1p has been described as the G $\beta$-subunit for the G $\alpha$-protein Gpa2 of a nutrient responsive cAMP/PKA signal transduction pathway that regulates pseudohyphal development in diploid and invasive/adhesive growth in haploid cells (Zeller et al., 2007). Like other G $\beta$-proteins, Asc1p binds to the GDP-bound form of Gpa2p and inhibits the GDP-GTP exchange on the G $\alpha$-protein (Zeller et al., 2007). A G $\gamma$-subunit of this pathway has not been identified so far.

The function of Asc1p cannot be exclusively restricted to a putative role as a G $\beta$. Asc1p belongs to the $5 \%$ most abundant proteins in the cell, and this extremely high abundance with approximately $6400 \mathrm{ppm}$ outreaches by far the abundance of Gpa2p with about $60 \mathrm{ppm}$ (PaxDb, Wang et al., 2012; Wang et al., 2015a). The first published study on S. cerevisiae Asc1p already described the localization of the protein to the 40S subunit of the ribosome based on its detection in the respective fractions of sucrose-gradients (Chantrel et al., 1998). Subsequent studies corroborated this initial finding, and nowadays the crystal structures of the Tetrahymena thermophila $40 \mathrm{~S}$ subunit and the S. cerevisiae $80 \mathrm{~S}$ ribosome are available providing information on the precise and highly conserved position of Asc1p at the head of the 40S subunit next to the mRNA exit tunnel (Figure 2, Sengupta et al., 2004; Ben-Shem et al., 2011; Rabl et al., 2011). Asc1p contacts the ribosomal proteins Rps3, Rps16, and Rps17 and interacts with the phosphate backbone and nucleotide bases of helix 39 and helix 40 of 
the 18S rRNA (Rabl et al., 2011). The binding of Asc1p to ribosomes even under high-salt concentrations (Link et al., 1999; Inada et al., 2002; Rabl et al., 2011) and its frequent presence in cryo-electron microscopy maps of ribosomes implicate that most or even all functional ribosomes in the cell contain Asc1p (Nilsson et al., 2004; Sengupta et al., 2004). Furthermore, the ASC1 gene shares typical characteristics of ribosomal genes: Like $66 \%$ of the $S$. cerevisiae ribosomal genes, the ASC1 open-reading frame is interrupted by an intron (Chantrel et al., 1998; Link et al., 1999). In contrast, only $3.7 \%$ of all S. cerevisiae genes contain an intron (Link et al., 1999). The 273 bp large intron is positioned at bp 538 to 810 of the 1233 bp large ASC1 gene and comprises the SNR24 gene that codes for the small nucleolar RNA (snoRNA) U24 (Chantrel et al., 1998).

As part of a ribonucleoprotein complex, snoRNA U24 directs the site-specific 2'-O-ribosemethlyation of $25 \mathrm{~S}$ pre-ribosomal RNA (Kiss-László et al., 1996). Another feature that is shared by $A S C 1$ and ribosomal genes is the regulation of its mRNA synthesis by the transcription factors Fhl1p and Ifh1p (Kleinschmidt et al., 2006). Despite the characterization of Asc1p as a core component of the 40S ribosomal subunit, a fraction of ribosome-unbound Asc1p seems to exist. It has been shown, for instance, that a ribosome-free form of Asc1p arises in the stationary growth phase of yeast cultures (Baum et al., 2004) indicating that growth conditions and extracellular signals could shift Asc1p to or away from the ribosome.
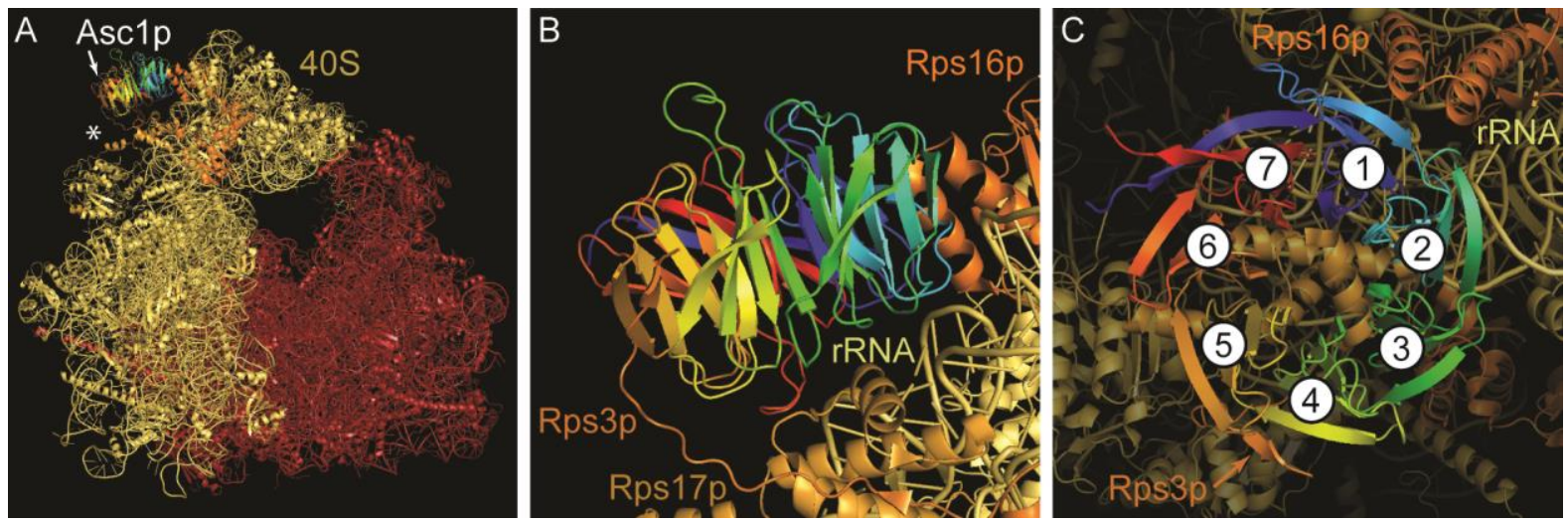

Figure 2. Localization of Asc1p to the ribosome.

(A) Localization of Asc1p to the small 40S subunit of the ribosome next to the mRNA exit tunnel. Asc1p is colored using a gradient from blue (N-terminus) to red (C-terminus). The ribosomal proteins and rRNA of the small 40S subunit are colored in different shades of yellow and orange. The ribosomal proteins and rRNA of the large 60S subunit are colored in red. The mRNA exit tunnel is indicated with an asterisk. (B) Close-up and side-view on Asc1p bound to the ribosome. Asc1p interacts with ribosomal proteins Rps3, Rps16, and Rps17 as well as with rRNA. (C) Close-up and bottom-view of Asclp bound to the ribosome. According to the definition for $\beta$-propeller proteins, Asc1p faces the ribosome with its top side. The $\beta$-propeller blades are labeled from 1 to 7 . Figures were generated with the PyMOL Molecular Graphics System software on the basis of the PDB file 4V88 (Ben-Shem et al., 2011). 
This also implicates the presence of Asc1p-deficient ribosomes. A simultaneous interaction of Asc1p with the Ga Gpa2p and the ribosome was disfavored due to sterical hindrance (Coyle et al., 2009), however, Asc1p might dissociate from Gpa2p upon activation of its associated transmembrane receptor Gpr1p and translocate to the ribosome for signal transmission to the regulation of mRNA translation. The viability of yeast ASC1 deletion strains shows that the protein is not essential for mRNA translation in general. Instead, it might link signal transduction to the translational regulation of specific groups of transcripts (Rachfall et al., 2013).

\subsection{Asc1p orthologues within the eukaryotic domain}

Asc1p is a highly conserved eukaryotic protein with about $52 \%$ amino acid sequence identity to RACK1 of Homo sapiens and $46 \%$ to RACK1A of Arabidopsis thaliana. Comparison of the crystal structures of the orthologous Asc1/RACK1 proteins of S. cerevisiae, H. sapiens, A. thaliana, and T. thermophila shows that this high conservation also manifests on the structural level (Ullah et al., 2008; Coyle et al., 2009; Rabl et al., 2011; Ruiz Carrillo et al., 2012). Besides studies on S. cerevisiae Asc1p, there are plenty of publications on its orthologues from different organisms ranging from fungi, plants, and insects, to mammals. Alterations in Asc1p/RACK1 abundance affect fundamental cellular processes. Deletion of the $S$. cerevisiae ASC1 gene leads to diverse phenotypes comprising decreased resistance against cell wall stress, absence of haploid adhesive growth and diploid pseudohyphae formation, cold and heat sensitivity, and reduced respiratory activity (Gerbasi et al., 2004; Kleinschmidt et al., 2006; Valerius et al., 2007; Rachfall et al., 2013). In S. cerevisiae, Asc1p was first identified in a screen for gene deletions that suppress the growth defect of hemedepleted $\Delta$ hapl $(\Delta c y p 1)$ cells. Hap1p is a transcription factor required for oxygen and hemedependent regulation of gene expression. Thus, the protein was termed Absence of growth suppressor of Hap1p/Cyp1p 1 (Asc1p, Chantrel et al., 1998).

\subsubsection{Asc1p orthologues in other fungi}

Orthologues of Asc1p have been studied in various other ascomycetes besides S. cerevisiae. In the fission yeast Schizosaccharomyces pombe, the deletion of the ASC1 orthologous gene cpc2 increases heat sensitivity and delays cell division at $\mathrm{G}_{2}$ as well as conjugation and meiosis due to failure of the cells to accumulate in $\mathrm{G}_{1}$ upon respective stimuli (McLeod et al., 2000). Homozygous $\Delta a s c 1$ strains of the opportunistic human pathogen Candida albicans are deficient in hyphal development as well as adhesive and invasive growth (Kim et al., 2010; 
Liu et al., 2010). The morphological transition of $C$. albicans between yeast, pseudohyphal, and hyphal growth forms is considered as essential for the pathogenicity of the fungus, and in line with this, $A S C l$ deleted $C$. albicans strains show strongly reduced pathogenicity in mouse infection models (Kim et al., 2010; Liu et al., 2010). In the filamentous fungi Aspergillus nidulans and Aspergillus fumigatus, the deletion of the $c p c B$ gene results in defects in spore germination, hyphal growth, and asexual conidiospore formation. Moreover, in A. nidulans the sexual development of cleistothecia was impaired resulting in the absence of ascospore formation (Kong et al., 2013). Furthermore, A. fumigatus shows decreased virulence in an immunosuppressed mouse infection model for invasive aspergillosis (Cai et al., 2015).

In the basidiomycete and opportunistic human pathogen Cryptococcus neoformans, absence of the Asc1p orthologue Gib2p results in reduced growth at $37^{\circ} \mathrm{C}$ as well as decreased pathogenicity in a mouse infection model (Wang et al., 2014). Absence of the Asc1p orthologue Rak1 also decreases the virulence of the phytopathogenic basidiomycete Ustilago maydis. Deletion of rakl leads to altered cell morphology, longer doubling times, and increased sensitivity to cell wall stress, and it impairs the mating process, which is a prerequisite for plant infection (Wang et al., 2011).

\subsubsection{Plant RACK1 proteins}

Plants can possess more than one RACK1 orthologue within their genome. A. thaliana harbors three versions named from $A$ to $C$ with approximately $87 \%$ identity and $94 \%$ similarity between RACK1A and each of the other two orthologues on the level of the amino acid sequence (Chen et al., 2006). All three genes are ubiquitously expressed in the plant, but differ in their expression levels with RACK1A showing the highest and RACK1C the lowest transcript level in various tissues (Chen et al., 2006; Guo and Chen, 2008). A loss-of-function rackla mutant shows various developmental defects in seed germination, leaf production, and flowering as well as deregulation of hormone responses (Chen et al., 2006). In contrast, lossof-function racklb and racklc single as well as double mutants have no obvious developmental defects (Guo and Chen, 2008). Yet, double mutants lacking RACK1A in combination with one of the other two RACK1s show synergistic effects. Triple mutants missing all three orthologues mostly die off in soil, and those that survive cannot reach maturity. Overexpression of $R A C K 1 B$ or $R A C K 1 C$ rescues the phenotype of a rackla mutant implicating that the three orthologues are functionally redundant and only differ in the regulation of their expression (Guo and Chen, 2008). The rice species Oryza sativa harbors two RACK1 orthologues that are named according to the nomenclature in A. thaliana 
$R A C K 1 A$ and $R A C K 1 B$ (Zhang et al., 2014). Also in rice, the RACK1A gene appears to be more prominently expressed than the $R A C K 1 B$ gene (Zhang et al., 2014). It is involved in seed germination and regulation of responses to exogenous abscisic acid, a plant hormone, and to $\mathrm{H}_{2} \mathrm{O}_{2}$, a reactive oxygen species with cell damaging properties that also serves in cellular signal transduction (Zhang et al., 2014).

\subsubsection{RACK1 in metazoan organisms}

Like in plants, RACK1 is almost ubiquitously expressed in mammals (Chou et al., 1999; Ruan et al., 2012). Metazoan RACK1 is an essential gene, and its depletion leads to lethality in early stages of embryogenesis. Homozygous RACK1 depletion in mouse results in lethality at gastrulation (Volta et al., 2012). Heterozygous adult mice with only one functional RACK1 copy in their genome show no differences in RACK1 levels and are viable. However, they show skin pigmentation defects, and eleven days old female pups show a transient growth defect (Volta et al., 2012). At this developmental stage, RACK1 expression differs in the brain and liver. In Xenopus laevis, knockdown of $R A C K 1$ results in impaired neuronal tube closure during embryogenesis through defects in convergent extension movements (Wehner et al., 2011). In zebrafish Danio rerio, rackl knockdown also impairs convergent extension during gastrulation, as well as oriented cell division and cellular polarization (Li et al., 2011). Depletion of RACK1 through RNA interference in C. elegans leads to developmental-timing delay, and its loss-of-function is embryonic lethal (Jannot et al., 2011). In Drosophila melanogaster, RACK1 is required throughout embryogenesis, larval development, and pupal stages (Kadrmas et al., 2007). A small percentage of RACK1-deficient flies can reach adulthood, but they exhibit severe phenotypes including sterility (Kadrmas et al., 2007). Silencing of RACK1 expression specifically in adult flies using a thermosensitive Gal80 system did not affect viability, but reduced the longevity by 20\% (Majzoub et al., 2014). Furthermore, the depletion of RACK1 in cell lines of different organisms does not cause lethality indicating that RACK1 is only essential during embryogenesis (Majzoub et al., 2014). A role of RACK1 already in the first stages of embryogenesis has been implicated from the high levels of RACK1 in ovaries as well as from the high abundance of maternally inherited RACK1 transcripts in zygotes of D. melanogaster (Vani et al., 1997; Kadrmas et al., 2007). Analysis of mutant flies devoid of RACK1 in the germ line revealed that the maternally inherited transcripts are indeed essential for the earliest steps of embryogenesis, and their absence cannot be compensated by zygotic RACK1 (Kadrmas et al., 2007). 
Beyond its pivotal role in development, RACK1 seems to play a decisive role in cancer proliferation. Aberrant RACK1 expression is associated with different cancer types and was proposed as a biomarker for diagnosis and prognosis of the clinical outcome of certain cancer types (Wang et al., 2009; Cao et al., 2010). The importance of RACK1 in cancer development seems to arise from its role in fundamental cellular processes, such as cell proliferation, cell spreading (Hermanto et al., 2002), angiogenesis (Berns et al., 2000), and apoptosis (Ruan et al., 2012).

\subsubsection{ASC1/RACK1 gene expression correlates with ribosomal genes}

Expression of plant $R A C K 1$ genes has been reported to be regulated by different hormones. Already the first described plant $R A C K 1$ was identified in a screen for auxin-responsive genes in the tobacco BY-2 cell line (Ishida et al., 1993). In A. thaliana, all three RACK1 genes are down-regulated in their expression levels upon treatment with abscisic acid (Guo et al., 2009), whereas $O$. sativa RACK1 mRNA levels increase in response to the same hormone as well as upon treatment with jasmonate and auxin (Nakashima et al., 2008). Guo and colleagues (2011) reported that about $80 \%$ of proteins that are co-regulated in their expression with $R A C K 1$ are ribosomal genes. Similarly, increased expression of ASC1 in S. cerevisiae upon growth with ethanol instead of glucose as sole carbon source correlated with increased expression of RPS26 that encodes a protein of the small ribosomal subunit (Kleinschmidt et al., 2006). Also, the biosynthesis of human RACK1 appears to correlate with that of other ribosomal genes. The association of human RACK1 mRNA to polysomes and thus its translation efficiency in response to serum deprivation as well as serum stimulation of human HeLa cells resembles that of the mRNA of the ribosomal protein RPS6 (Loreni et al., 2005). Additionally, the RACK1 mRNA shows similarity to other ribosomal mRNAs since it starts with an oligopyrimidine sequence and ends with a short 3' untranslated region (3'UTR) of 45 nucleotides (Loreni et al., 2005).

\subsubsection{Orthologous genes complement $A S C 1 / R A C K 1$-deficiency across phyla}

As outlined in the previous chapters, misregulation of $A S C 1 / R A C K 1$ expression ranging from deletion to its overexpression in various eukaryotic model organisms interferes with vital cellular events and manifests in diverse and severe phenotypes. It has been repeatedly shown that phenotypes caused by the deletion of ASC1/RACK1 can be complemented by the expression of orthologues from other species underlining its high conservation within the eukaryotic domain. Rat RACK1 expressed in S. cerevisiae $\Delta$ ascl cells can be detected in 
ribosomal fractions of sucrose density gradients, and it partially complements the temperature sensitivity phenotype of the $\operatorname{sascl}$ strain at $37^{\circ} \mathrm{C}$ (Gerbasi et al., 2004). Likewise, expression of rat RACK1 in A. nidulans strains deficient in the ASC1/RACK1 orthologous $c p c B$ gene complements defects in sexual development (Hoffmann et al., 2000). Expression of each of the three A. thaliana orthologues in a diploid S. cerevisiae $\Delta$ ascl/Dascl strain restored the ability of this strain to form pseudohyphae under nitrogen starvation (Guo et al., 2011), and the expression of $U$. maydis Rak1 can complement the adhesive growth defect of a haploid S. cerevisiae $\Delta$ ascl strain (Wang et al., 2011). Expression of human RACK1 in X. laevis rescues the defect in neuronal tube closure during embryogenesis upon knockdown of the endogenous RACK1 mRNA (Wehner et al., 2011).

\subsection{Described molecular function of Asc1p/RACK1}

Many studies addressed the molecular function of Asc1p/RACK1 that underlies the described phenotypes (chapter 1.3). The following chapter will give an introduction to the current knowledge about the role of the protein in mRNA translation, signal transduction, and protein degradation.

\subsubsection{Asc1p/RACK1 as a regulator of mRNA translation}

The characterization of Asc1p as an integral component of the small ribosomal subunit suggests a role of the protein in the process of mRNA translation. S. cerevisiae Asc1p seems to act as a repressor of translation as shown in vitro (Gerbasi et al., 2004) and in vivo (Chiabudini et al., 2014). In vitro translation assays using cell extracts of ASCl wild-type and deletion strains and different templates including capped as well as uncapped luciferase mRNA and wild-type poly(A) mRNA revealed increased translation of mRNAs in extracts from $\Delta a s c 1$ cell. Yet, the addition of exogenous and recombinantly-expressed Asc1 protein to the samples of the in vitro translation assay did not repress translation activity (Gerbasi et al., 2004). In vivo, absence of Asc1p reduces the average ribosome transit time, which is required by a ribosome for elongation and termination of translation, by approximately $30 \%$ (Chiabudini et al., 2014). In contrast, overexpression of RACK1 in the human hepatocellular carcinoma derived cell line Huh7 was reported to promote protein biosynthesis in metabolic labeling studies with $\left[{ }^{35} \mathrm{~S}\right]$-methionine (Ruan et al., 2012). Using firefly and renilla luciferase reporter constructs it could be further shown that RACK1 promotes cap-dependent as well as internal ribosomal entry site (IRES)-mediated translation (Ruan et al., 2012). A role of RACK1 as a promoter of translation was also deduced from experiments measuring the 
translation of firefly luciferase reporter constructs within cell extracts from wild-type and Rack1-null thoracic muscle cells of D. melanogaster (Belozerov et al., 2014). Embryonic fibroblasts of heterozygous mice that carry only one functional copy of RACK1 showed reduced stimulation of translation through treatment with insulin or phorbol 12-myristate 13-acetate (Volta et al., 2012). Liver cells of 16 days old RACK1 heterozygous mice show a mild accumulation of $80 \mathrm{~S}$ ribosomes, and the skin pigmentation phenotype of the adult mutant mice resembles that of RPL24 mutants (Volta et al., 2012). In conclusion, Asc1p/RACK1 seems to fulfill both a repressive as well as a promoting effect on general mRNA translation rates depending on the cellular context.

\subsubsection{S. cerevisiae Asc1p in co-translational quality control and reading frame maintenance}

In $S$. cerevisiae, Asc1p was proposed to function in mRNA quality control systems that act during translation (Kuroha et al., 2010; Matsuda et al., 2014). Different surveillance systems recognize aberrant mRNAs and guide their degradation as well as the clearance of the corresponding peptide products (reviewed in Inada, 2013). Asc1p promotes nascent polypeptide-dependent translation arrest that is caused by translation of stretches of the basic amino acids lysine and arginine encoding sequences within reporter constructs (Kuroha et al., 2010; Brandman et al., 2012; Matsuda et al., 2014). Stretches of lysine in the growing peptide chain can arise, for instance, when the mRNA lacks a stop codon and translation precedes to the poly(A) tail of the mRNA because AAA triplets code for lysine (Lu and Deutsch, 2008). The positively charged amino acids in the growing peptide chain appear to slow down translation through interaction with the negatively charged ribosome tunnel (Lu and Deutsch, 2008). Asc1p further promotes translation arrest at repeats of rare or inefficiently decoded CGA triplets and is required for the co-translational degradation of arrested products via the proteasome and the endonucleolytic cleavage of the corresponding mRNA (Kuroha et al., 2010; Letzring et al., 2013; Matsuda et al., 2014). Furthermore, deletion of ASC1 causes frameshifting at the inefficiently translated CGA repeats, a phenomenon that is not observed in wild-type cells (Wolf and Grayhack, 2015). The inefficient translation of the arginine encoding CGA triplet is due to wobble decoding and can be suppressed through expression of an anticodon mutated tRNA that perfectly pairs the CGA codon (Letzring et al., 2010). Frameshifting at CGA codons in ASC1 deleted cells becomes more favored with an increasing distance of the codon from the translational start site (Wolf and Grayhack, 2015). Also, the afore mentioned inhibitory effect of CGA codon repeats on translation depends on a certain 
distance from the translation start site and seems to be independent of Asc1p when the CGA repeats lie close to the start codon. Pairs of two adjacent CGA codons are found in 26 genes of S. cerevisiae (Wolf and Grayhack, 2015), thus Asc1p might regulate the translation of specific mRNAs. Furthermore, Asc1p was reported to affect the translation in dependence of the 5'UTRs of mRNAs (Rachfall et al., 2013).

\subsubsection{Asc1p/RACK1-dependent phosphorylation of translation factors}

The molecular mechanisms underlying the effects of Asc1p/RACK1 on mRNA translation remain mostly elusive, but some studies provided starting points for a better understanding of its function in protein biosynthesis. Experiments with mammalian cell lines showed that RACK1 localizes activated protein kinase PKC $\beta$ II to ribosomes leading to phosphorylation of the eukaryotic translation initiation factor $\underline{6}$ (eIF6). This modification induces the release of eIF6 from the 60S ribosomal subunit followed by $80 \mathrm{~S}$ assembly and translation initiation (Ceci et al., 2003). RACK1-mediated positioning of activated PKC $\beta I I$ is also required for phosphorylation of eIF4E shown in human hepatocellular carcinoma cell lines (Ruan et al., 2012). Blocking the interaction between PKC $\beta I I$ and RACK1 reduces stimulation of translation through activated PKC in human HeLa and Huh7 cell lines (Grosso et al., 2008; Ruan et al., 2012). An interaction of the A. thaliana RACK1 proteins with the two orthologous eIF6 proteins A and B was also shown and implicates a possible conservation of this mechanism among different eukaryotic species (Guo et al., 2011). Experiments with human embryonic kidney cell lines HEK293 showed that localization of c-Jun N-terminal kinase (JNK) to ribosomes is also mediated by RACK1 leading to phosphorylation of S205 and S358 of the elongation factor eEF1A2, one of the two mammalian eEF1A isoforms (Gandin et al., 2013a). Phosphorylation of eEF1A2 at these residues enhances its association with newly synthesized and misfolded polypeptides at the ribosome and their degradation by the proteasome (Gandin et al., 2013a).

So far, it has not been shown that $S$. cerevisiae Asc1p recruits kinases to the translational machinery. However, deletion of $A S C 1$ results in increased phosphorylation of the translation initiation factors eIF2 $\alpha$ and eIF4A as well as Ssz1p, a component of the ribosome-associated complex (Valerius et al., 2007). Absence of Asc1p affects phosphorylation of the $\alpha$-subunit of eIF2 at residue S51 (Valerius et al., 2007). This modification inhibits eIF2B-mediated GDP to GTP exchange at eIF2, a prerequisite for binding of the methionine-carrying initiator tRNA $\left({ }^{\mathrm{Met}} \mathrm{tRNA}_{\mathrm{i}}\right)$ to the initiation factor. Consequently, the amount of the ternary complex consisting of eIF2, GTP, and the ${ }^{\text {Met }}$ RNA $_{i}$ decreases, when phosphorylation of eIF2 $\alpha$ at S51 
increases (Dever et al., 1992). Since the ternary complex is essential for translational initiation, overall cellular protein biosynthesis is reduced upon elevated eIF2 $\alpha$ phosphorylation. The phosphorylation of eIF $2 \alpha$ is thus a key factor in the regulation of mRNA translation, and it is mediated by the sensor kinase Gcn2p in response to nutrient availability (Dever et al., 1992). The kinase activity of Gcn2p is induced through binding of uncharged tRNAs that are tRNAs carrying no amino acid. The abundance of these uncharged tRNAs reflects the availability of amino acids (Wek et al., 1995). In $\Delta$ ascl cells, phosphorylation of eIF2 $\alpha$ at S51 is increased even without amino acid starvation induced by the histidine analogon $\underline{3}$-amino-1,2,4-triazole (3-AT, Valerius et al., 2007). In contrast, absence of Cpc2p in $S$. pombe leads to decreased phosphorylation of eIF2 $\alpha$ in response to 3-AT (Tarumoto et al., 2013). In line with this, the autophosphorylation and thus activation of the Gcn2p kinase at residue T818 is reduced in $\Delta c p c 2$ cells under the same conditions (Tarumoto et al., 2013).

\subsubsection{Asc1p/RACK1-dependent recruitment of proteins to the ribosome}

Besides a putative role of Asc1p as a docking site for kinases at the ribosome, it could also localize other proteins to the translational machinery. Asc1p was shown to interact with the c-subunit of eIF3, Nip1p (Kouba et al., 2012). Furthermore, the C.neoformans Asc1p orthologue Gib2p interacts with eIF4A (Wang et al., 2014), while Trypanosoma brucei RACK1 associates with eEF1A most likely in an indirect way (Regmi et al., 2008; Choudhury et al., 2011).

Asc1p/RACK1 was further shown to associate with mRNA-binding proteins in different organisms. S. cerevisiae Asc1p interacts with the mRNA-binding protein Scp160 and is required for its localization to ribosomes (Baum et al., 2004; Coyle et al., 2009). Both proteins can associate with the translation inhibitor Eap1p and the GYF domain containing protein

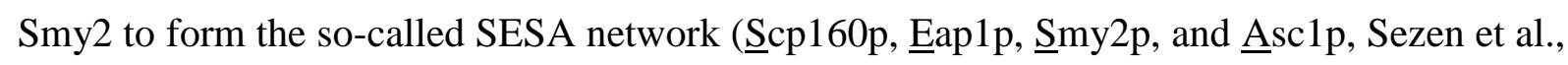
2009). The complex regulates the translation of the POM 34 mRNA that encodes an integral membrane protein of the nuclear pore complex. Defects in spindle pole body duplication during cytokinesis promote the SESA-mediated inhibition of POM34 mRNA translation most likely to relieve an inhibitory effect of Pom34p on spindle pole body duplication (Sezen et al., 2009).

The Asc1p orthologue of $S$. pombe Cpc2p was further shown to interact with the mRNAbinding protein Nrd1 (Jeong et al., 2004). Nrd1p represses Ste11p-regulated genes required for sexual differentiation and binds and stabilizes $c d c 4$ mRNA that encodes a myosin light 
chain crucial for cytokinesis (Tsukahara et al., 1998; Satoh et al., 2009). In neuronal mammalian cells, RACK1 binds to the mRNA-binding protein ZBP1 within transport ribonucleoprotein complexes (RNPs, Ceci et al., 2012). These RNA granules contain translationally repressed mRNAs that are transported along dendrites or axons, such as the ZBP1-bound $\beta$-actin mRNA. At their destination, RACK1 is required for the recruitment of the activated Src-kinase to the ZBP1/ $\beta$-actin mRNA complex for phosphorylation of ZBP1 and the subsequent release and translation of the $\beta$-actin mRNA (Ceci et al., 2012).

Neuronal RNPs are related to processing bodies (P-bodies) and stress granules that contain untranslated mRNAs and increase in their abundance upon inhibition of translation initiation (Decker and Parker, 2012). P-bodies and stress granules form foci in the cytoplasm that are localized adjacent to each other or partially overlap, and RNPs seem to cycle between these two structures and actively translating polysomes. P-bodies contain proteins involved in mRNA decay and repression of translation (Decker and Parker, 2012). Deletion of ASC1 in S. cerevisiae prevents P-body formation in response to DNA replication stress induced by hydroxyurea, but not in response to osmotic stress/starvation (Tkach et al., 2012). In contrast to P-bodies, stress granules contain different translation initiation factors and components of the small ribosomal subunit including RACK1 as shown for human HeLa cells subjected to certain stress conditions (Arimoto et al., 2008; Decker and Parker, 2012).

\subsubsection{RACK1's function in IRES-dependent translation initiation and the miRNA pathway}

In D. melanogaster and in the Huh7.5.1 cell line, silencing of RACK1 decreases propagation of viruses belonging to the Dicistroviridae and Flaviviridae families, respectively, due to the requirement of RACK1 for IRES-mediated mRNA translation (Majzoub et al., 2014). Notably, RACK1 is not required for the activity of those IRES containing mRNAs that bind to the $40 \mathrm{~S}$ subunit and recruit the $60 \mathrm{~S}$ subunit independently of translation initiation factors. The Hepatitis $\mathrm{C}$ virus of the Flaviviridae family causes liver disease and hepatocellular carcinoma. Thus, RACK1 might evolve as a target for antiviral therapy (Majzoub et al., 2014). RACK1 has further been described to act in the miRNA pathway of $C$. elegans, H. sapiens, and A. thaliana, but its function seems to differ between the different organisms (Speth et al., 2013). In metazoans and plants, small non-coding micro RNAs (miRNAs) play an important role in gene silencing on a post-transcriptional level. The miRNA pathway requires processing of primary miRNAs by the Dicer enzyme into 20 to 22 nucleotide long

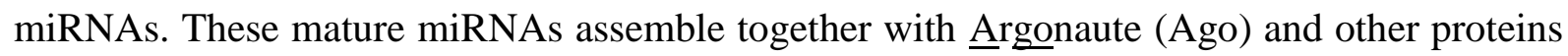


into a miRNA-induced silencing complex (miRISC). The complex is directed by the miRNA to target mRNAs via base-pairing to regulate their translation or degradation. Experiments with human hepatoma cell lines showed that RACK1 interacts with Ago2 and with the KHtype splicing regulatory protein that associates with the Dicer complex and is involved in miRNA maturation (Otsuka et al., 2011). RACK1 was proposed to function after miRNA maturation and might be involved in the recruitment of mature miRNA to the RISC (Otsuka et al., 2011). Another study reported that RACK1 interacts with the miRISC in C. elegans and human HeLa cells (Jannot et al., 2011). The localization of the miRISC to translating ribosomes is reduced upon depletion of RACK1 resulting in increased expression of specific miRNA targets (Jannot et al., 2011). In A. thaliana, however, RACK1 deficiency reduces the level of a large number of primary miRNAs (Speth et al., 2013). RACK1 interacts with the protein SERRATE, a protein involved in miRNA production. RACK1 is further found in complex with the miRISC component AGO1 suggesting more than one role of plant RACK1 in the miRNA pathway (Speth et al., 2013).

\subsubsection{Asc1p/RACK1 in signal transduction pathways}

Asc1p/RACK1 shows high similarity to the G $\beta$-subunits of heterotrimeric G-proteins and was described as the G $\beta$-subunit of the G $\alpha$-protein Gpa2. In other organisms, Asc1p/RACK1 has been associated to G-protein-mediated signaling as well, but it also functions in other signal transduction pathways and seems to provide a central hub to link different pathways. The following chapters describe the function of Asc1p/RACK1 in cellular signaling in different organisms ranging from yeast to man.

\subsubsection{Role of Asc1p and its orthologues in signal transduction pathways in fungi and plants}

Immunoprecipitation experiments showed that $S$. cerevisiae Asc1p can be co-purified with the adenylyl cyclase Cyr1p of the cAMP/PKA pathway and with the mitogen-activated protein kinase kinase kinase kinase (MAP4K) Ste20p (Figure 3, Zeller et al., 2007). Asc1p was suggested to inhibit the activity of both enzymes: Cyrlp catalyzes the formation of cAMP from ATP, and absence of Asc1p enhances cAMP levels upon stimulation of starved cells with glucose. Phosphorylation and activation of the Ste20p down-stream MAPK Kss1p is increased in $\Delta$ ascl cells compared to wild-type cells (Zeller et al., 2007). Additionally, Asc1p was identified in complex with the MAPK Slt2p of the signal transduction pathway that 


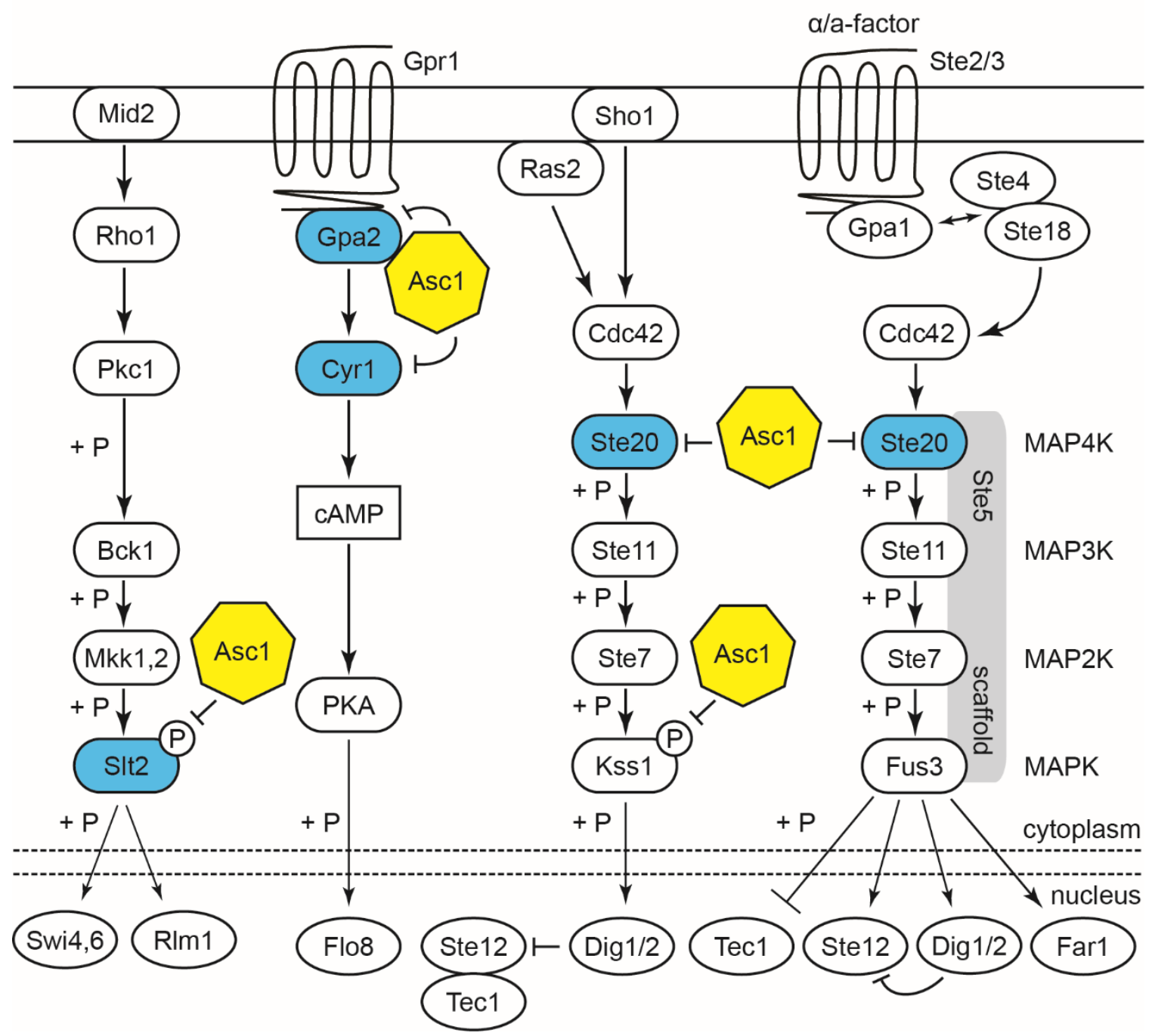

Figure 3. Model on Asc1p's function in the cAMP/PKA pathway and MAPK cascades in S. cerevisiae.

The cAMP/PKA pathway and the MAPK cascades regulating cell wall integrity, filamentous and adhesive growth, and mating are depicted. Proteins that were described to associate physically with Asc1p are colored in blue. Asc1p directly interacts with the G $\alpha$-protein Gpa2 of the cAMP/PKA pathway and was proposed to function as the G $\beta$-subunit. The interaction between Asc1p and Gpa2p inhibits the exchange of Gpa2p-bound GDP for GTP, a process that is required for activation of the $\mathrm{G} \alpha$-protein. Asc1p was further co-purified with the adenylyl cyclase Cyr1p and the MAP4K Ste20p and seems to inhibit the function of these proteins within their respective pathways (Zeller et al., 2007). Asc1p was also identified in complex with the MAPK Slt2p (Breitkreutz et al., 2010). The amount of phosphorylated MAPKs Slt2p and Kss1p is increased in $\Delta a s c 1$ cells (Chasse et al., 2006; Zeller et al., 2007). The general overview of the signal transduction pathways is adapted from the KEGG database (http://www.genome.jp/kegg).

regulates cell wall integrity (Breitkreutz et al., 2010). Phosphorylation of Slt2p was increased in $\Delta a s c 1$ cells indicating enhanced activity of this pathway (Figure 3, Chasse et al., 2006).

The Asc1p orthologue of the basidiomycete C. neoformans Gib2p was also identified to

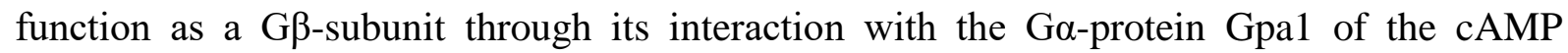




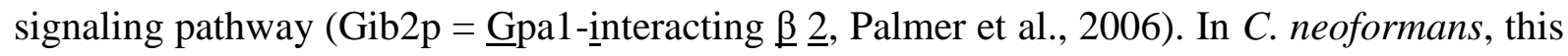
pathway also regulates the production of virulence factors, such as the antioxidant melanin and the polysaccharide capsule (Alspaugh et al., 1997). In contrast to well characterized G $\beta$-subunits of heterotrimeric G-proteins, Asc1p/RACK1 lacks the N-terminal coiled-coil domain important for interaction with G $\gamma$-subunits (Zeller et al., 2007; Ullah et al., 2008; Ruiz Carrillo et al., 2012). However, Gib2p of C. neoformans has been described to associate with the Gy-proteins Gpg1 and Gpg2 (Palmer et al., 2006). It further binds to the GTPase Ras1p that in turn interacts with the adenylyl cyclase Caclp (Wang et al., 2014). Whereas S. cerevisiae Asc1p was reported to inhibit the adenylyl cyclase Cyr1p, C. neoformans Gib2p positively affects cellular cAMP levels in Gpalp-deficient cells possibly through reducing the inhibitory effect of Ras1p on Cac1p (Zeller et al., 2007; Wang et al., 2014).

The three RACK1 proteins of A. thaliana have not been shown to function as G $\beta$ themselves, but they were reported to interact with the Gß-protein AGB1 (Cheng et al., 2015). Furthermore, they associate with all three components of the MAPK cascade and seem to provide a scaffold that links the heterotrimeric G-protein to MAPK signaling similar to Ste $5 p$ in the pheromone response pathway of $S$. cerevisiae. The plant G-protein/MAPK pathway is activated by pathogen secreted proteases placing RACK1 in the center of the plant innate immune response pathway (Cheng et al., 2015).

\subsubsection{Metazoan RACK1 - Receptor for activated C kinase 1}

Like the plant RACK1 proteins, mammalian RACK1 has also not been reported to function as a $G \beta$-subunit, but it interacts with the $G \beta_{1} \gamma_{1}$ dimer and to a lesser extent with the transducin heterotrimer $G \alpha_{t} \beta_{1} \gamma_{1}$ (Dell et al., 2002). Since RACK1 also interacts with other G $\beta \gamma$ isoforms, it might act as a general G $\beta \gamma$ binding partner (Chen et al., 2005). The association of RACK1 with G $\beta \gamma$ inhibits a specific subset of their signaling functions, whereas $\mathrm{G} \alpha$-mediated signal transduction seems to be RACK1-independent (Chen et al., 2004a).

Metazoan RACK1 has been described as an interaction partner for a plethora of different signaling molecules comprising proteins localized in the cytoplasm and nucleus as well as the cytosolic domains of membrane-spanning receptors (Li and Xie, 2015). RACK1 has versatile effects on its interaction partners. Similar to yeast Asc1p, RACK1 can influence the enzymatic activity of its associated proteins. Furthermore, it was shown that RACK1 shuttles proteins to their cellular target sites and that it scaffolds protein-protein interactions, thereby also interconnecting different signal transduction pathways and forming a central regulatory node (Gandin et al., 2013b; Li and Xie, 2015). 
Already some of the earliest studies on mammalian RACK1 characterized the protein as an interaction partner of activated PKC $\beta I I$ resulting in its name receptor for activated $\underline{\mathrm{C}} \underline{\text { kinase }} \underline{1}$ (Ron et al., 1994; Ron et al., 1995). RACK1 stabilizes the activated conformation of the kinase and shuttles the protein to its cellular target sites (Ron et al., 1994; Ron et al., 1999). The function of RACK1 has also been linked to other PKC isoforms (Liedtke et al., 2002; Robles et al., 2010; Wehner et al., 2011). The function of RACK1 in neuronal tube closure during embryogenesis of $X$. laevis, for example, seems to involve its interaction with $\mathrm{PKC} \delta 1$ in a membrane localized complex with PTK7, a regulator of planar cell polarity, and Dishevelled, a protein involved in Wnt signaling (Wehner et al., 2011).

\subsubsection{RACK1 links insulin-like growth factor 1 with integrin signaling}

In contrast to its effect on PKC, RACK1 acts as an inhibitor of the Src family of non-receptor protein tyrosine kinases specifically of Src and Fyn (Chang et al., 1998; Yaka et al., 2002; Adams et al., 2011). The release of Src from RACK1 involves the direct interaction of RACK1 with the insulin-like growth factor $\underline{1}$ receptor (IGF-1R), a transmembrane receptor and regulator of cell adhesion, migration, growth, and survival (Kiely et al., 2005). Upon activation of IGF-1R through its ligand IGF-1, RACK1 simultaneously binds the

without IGF-1

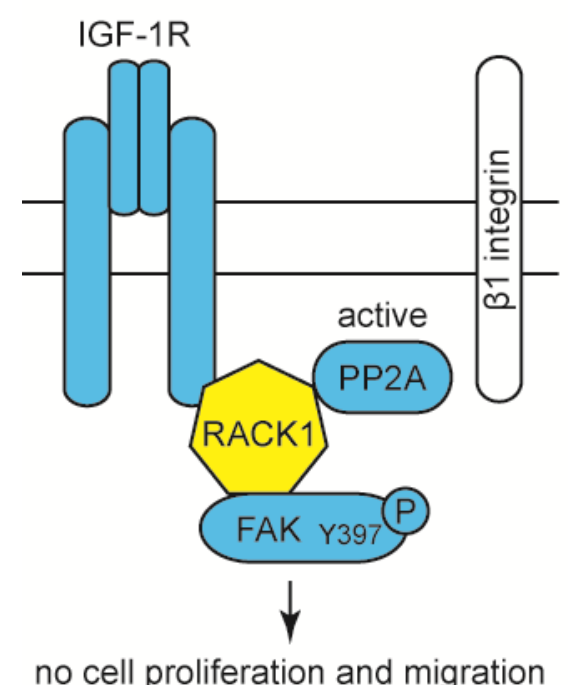

with IGF-1

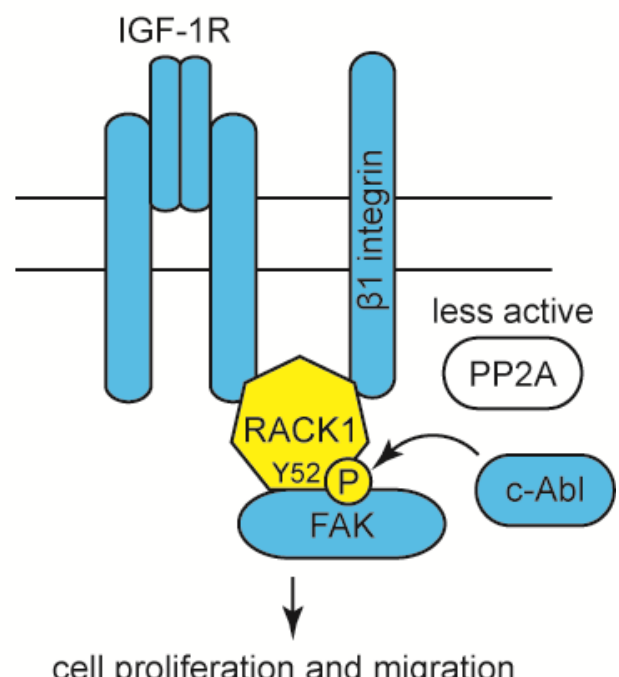

cell proliferation and migration

Figure 4. Model on the function of RACK1 in IGF-1R signal transduction.

In the absence of IGF-1, RACK1 recruits PP2A and phosphorylated FAK to the IGF-1R receptor. Upon binding of IGF-1 to its receptor, PP2A is released through binding of $\beta 1$ integrin to the same binding site on RACK1, and its activity is reduced. Furthermore, RACK1 is phosphorylated by the c-Abl kinase at Y52, a modification that stabilizes the interaction between RACK1 and FAK. This interaction is required for the dephosphorylation of FAK at Y397. These signaling events contribute to the induction of cell proliferation and migration. The model is adapted from Kiely et al. (2006 and 2009). Proteins that interact physically with RACK1 are colored in blue. 
transmembrane receptor $\beta 1$ integrin, another regulator of adhesion, thereby integrating signals coming from the two distinct receptors (Figure 4, Hermanto et al., 2002). Activation of IGF-1R leads to release of Src from RACK1 as well as to dissociation of protein phosphatase PP2A from the complex (Kiely et al., 2005; Kiely et al., 2006). Since PP2A and $\beta 1$ integrin bind to WD40 repeats 1 to 4 of RACK1 in a mutually exclusive manner, association of the membrane receptor with RACK1 excludes PP2A from the complex, thereby reducing its phosphatase activity (Kiely et al., 2006).

Stimulation of serum starved cells with IGF-1 further stabilizes the interaction of RACK1 with focal adhesion kinase (FAK) through phosphorylation of RACK1 at Y52 by the c-Abl kinase (Figure 4, Kiely et al., 2009). The interaction between RACK1 and FAK is essential for the IGF-1 triggered dephosphorylation of FAK at Y397 that is considered as a prerequisite for the induction of cell migration (Kiely et al., 2009). RACK1 also recruits other proteins to IGF-1R, such as phosphorylated Shc, IRS-1, IRS-2, and Shp2 (Kiely et al., 2005), and serves as an adaptor protein for Kindlin-3 and other signaling proteins at the $\beta 1$ integrin receptor (Feng et al., 2012).

\subsubsection{Metazoan RACK1 in cAMP/PKA signaling}

While the inhibitory effect of RACK1 on the Src kinase is relieved through IGF-1R signaling, RACK1-dependent inhibition of the non-receptor protein tyrosine kinase Fyn is abrogated through PKA induced dissociation of the two proteins (Figure 5, Yaka et al., 2003). Like yeast Asc1p, mammalian RACK1 is thus linked to cAMP/PKA signaling. In neurons of the hippocampus, RACK1 homodimers localize the Fyn kinase to the $\underline{N}$-methyl $\underline{D}$-aspartate (NMDA) receptor via interaction with the cytoplasmic tail of the receptor subunit NR2B (Yaka et al., 2002; Thornton et al., 2004). As long as RACK1 is part of the complex, phosphorylation of NR2B through Fyn is blocked (Yaka et al., 2002). However, upon activation of the cAMP/PKA pathway, RACK1 is released, and the phosphorylation takes place leading to increased ion channel activity of the NMDA receptor (Yaka et al., 2003). Homodimer formation of RACK1 enables its simultaneous association with NR2B and Fyn since both share the same interaction surface on RACK1 (amino acids 35 to 48, Thornton et al., 2004). This complex seems to be stabilized at membranes through interaction of RACK1 with the G $\beta$-subunit of a PAC1 receptor-associated heterotrimeric G-protein (Figure 5). Binding of pituitary adenylyl cyclase-activating polypeptide (PACAP) to the PAC1 receptor leads to dissociation of the G-protein that might in turn promote the release of RACK1 from the complex and thus Fyn-mediated phosphorylation of NR2B (Thornton et al., 2004). 
Activation of the cAMP pathway further leads to translocation of RACK1 to the nucleus, a

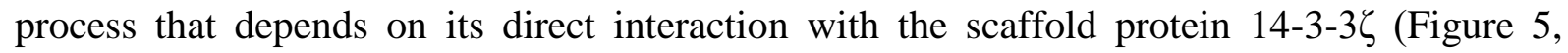
Neasta et al., 2012). In the nucleus, RACK1 localizes to the promoter region IV of the brainderived neurotrophic factor $(B D N F)$ gene possibly through interaction with histones $\mathrm{H} 3$ and H4 (Yaka et al., 2003; He et al., 2010). RACK1 induces the dissociation of the transcription repressor methyl- $\underline{\mathrm{Cp} G-b i n d i n g}$ protein $\underline{2}(\mathrm{MeCP} 2)$ from the promoter leading to histone $\mathrm{H} 4$ acetylation, chromatin remodeling and thus exon-specific transcription of BDNF (He et al., 2010).

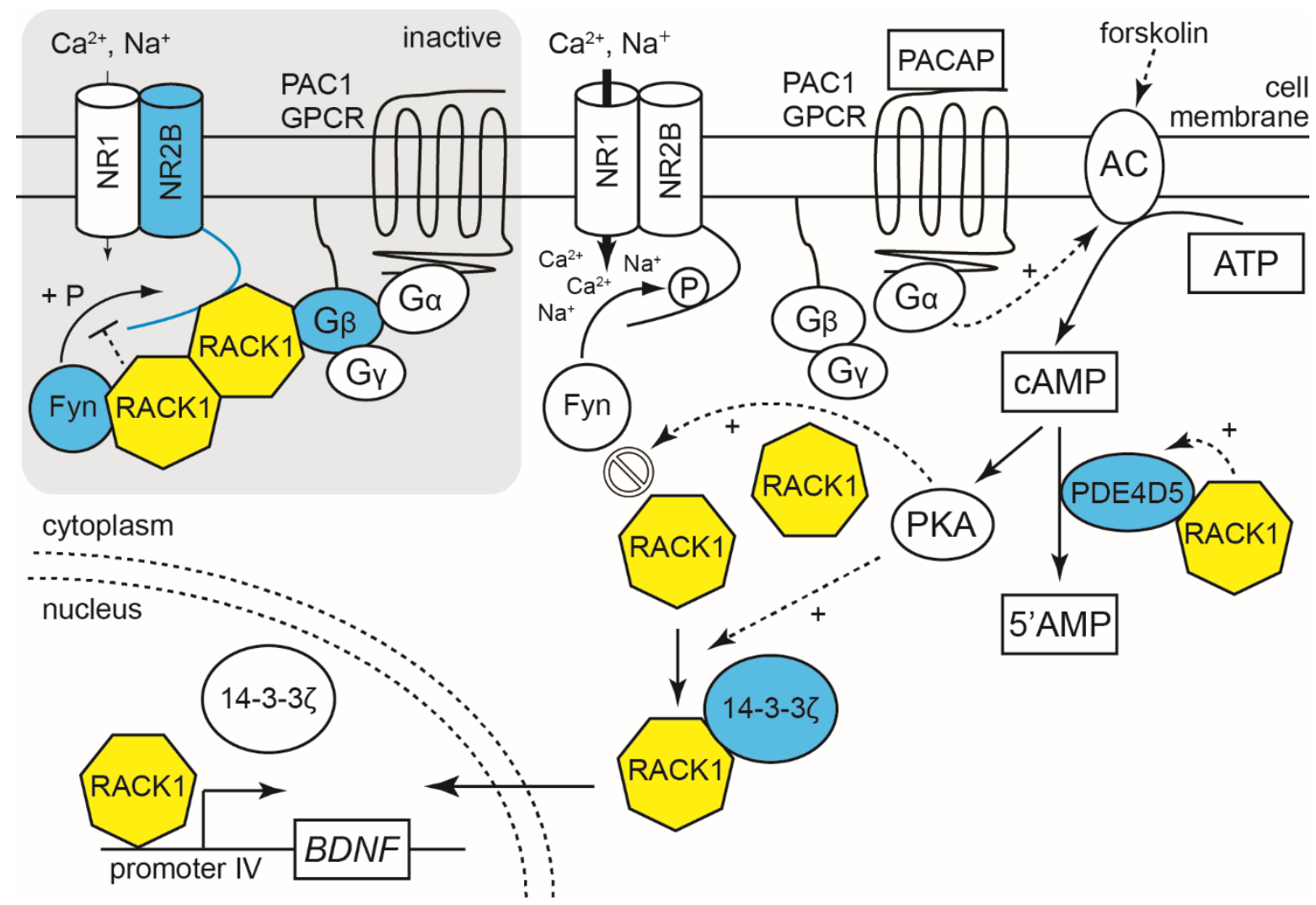

Figure 5. Model on the involvement of RACK1 in G-protein and cAMP/PKA signaling in metazoan.

RACK1 inhibits the phosphorylation of the NMDAR subunit NR2B by the Fyn kinase, thereby causing decreased channel activity. The interaction of RACK1 with G $\beta$ might stabilize the complex at the membrane. Binding of PACAP to the G-protein-coupled receptor (GPCR) PAC1 causes dissociation of the heterotrimeric G-protein into the $G \alpha$-subunit and the $G \beta \gamma$-heterodimer. This might destabilize the complex of NR2B, Fyn, and RACK1 releasing the inhibitory effect of RACK1 on Fyn and enabling phosphorylation of NR2B by the kinase and increasing the channels activity. RACK1's simultaneous interaction with NR2B and Fyn requires its homodimerization. Also, the activation of the adenylyl cyclase (AC) with forskolin and the subsequent activation of the cAMP/PKA pathway promote the release of RACK1 from the complex with NR2B and Fyn and thus the phosphorylation of NR2B. The figure is adapted from Yaka et al. (2003), Thornton et al. (2004), and the KEGG database. Additionally, the interaction between RACK1 and the scaffold protein $14-3-3 \zeta$ is depicted, which is promoted by active PKA and leads to translocation of RACK1 into the nucleus where it activates $B D N F$ transcription (Neasta et al., 2012). RACK1 seems to promote cAMP hydrolysis through increasing the affinity between PDE4D5 and its substrate (Bird et al., 2010). RACK1-interacting proteins are colored in blue. 
RACK1 is further involved in the cAMP pathway through its interaction with the phosphodiesterase (PDE) isoform PDE4D5 (Figure 5, Yarwood et al., 1999). PDEs provide the only mechanism to decrease cellular cAMP levels through catalyzing the hydrolysis of the molecule to 5'AMP. The PDE4 class of cyclic nucleotide PDEs is responsible for the majority of cAMP hydrolysis and is encoded by four genes (A-D) in mammals that provide different isoforms through alternative splicing (Conti et al., 2003). In membrane fractions of HEK293 cells, the interaction between RACK1 and PDE4D5 increases the affinity of the enzyme for its substrate cAMP (Bird et al., 2010).

\subsubsection{Function of RACK1 in MAPK cascades}

Like $S$. cerevisiae Asc1p, RACK1 was not only identified to be involved in the cAMP/PKA pathway, but also to interact with components of MAPK cascades (Figure 6). The MAPK p38b binds RACK1 in cells of D. melanogaster thoracic muscles and of the S2 cell line, and in vitro experiments showed that the MAPK can phosphorylate RACK1 (Belozerov et al., 2014). Genetic studies revealed that $\mathrm{p} 38 \mathrm{~b}$ and RACK1 act in a common pathway for the regulation of protein aggregate formation, life-span, and locomotor functions. In aging and stressed cells, activation of $\mathrm{p} 38 \mathrm{~b}$ seems to shift RACK1 to a ribosome-unbound pool that might repress translation (Belozerov et al., 2014).

In another study on the differentiation of bone marrow-derived macrophages (BMMs) into bone-resorbing osteoclasts, RACK1 was shown to act upstream in the p38 pathway as well (Lin et al., 2015). Mammalian RACK1 is required for p38 activation in response to the cytokine receptor activator of nuclear factor $\underline{\kappa} \mathrm{B}(\mathrm{NF}-\kappa \mathrm{B})$ ligand (RANKL) to induce osteoclast formation (Lin et al., 2015). The RANKL receptor RANK associates at its cytoplasmic domain with the E3 ubiquitin ligase tumor necrosis factor receptor-associated factor $\underline{6}$ (TRAF6, Galibert et al., 1998). Upon ligand binding to RANK, TRAF6 recruits the MAP3K transforming growth factor- $\beta$ activated kinase $\underline{1}$ (TAK1) via an adaptor protein to the complex leading to its activation (Mizukami et al., 2002). Experiments with HEK293T cells revealed that RACK1 mediates the interaction between the TRAF6-TAK1 complex and the p38-targeting MAP2K MKK6 that results in increased downstream phosphorylation of p38 (Figure 6, Lin et al., 2015). Treatment of bone marrow-derived macrophages with RANKL increases RACK1 levels depending on a putative binding site for the transcription factor and target of RANK-signaling NF- $\kappa \mathrm{B}$ within the promoter of the RACK1 gene (GNB2L1). Overexpression of RACK1 induces p38 phosphorylation and enhances RANKL- 


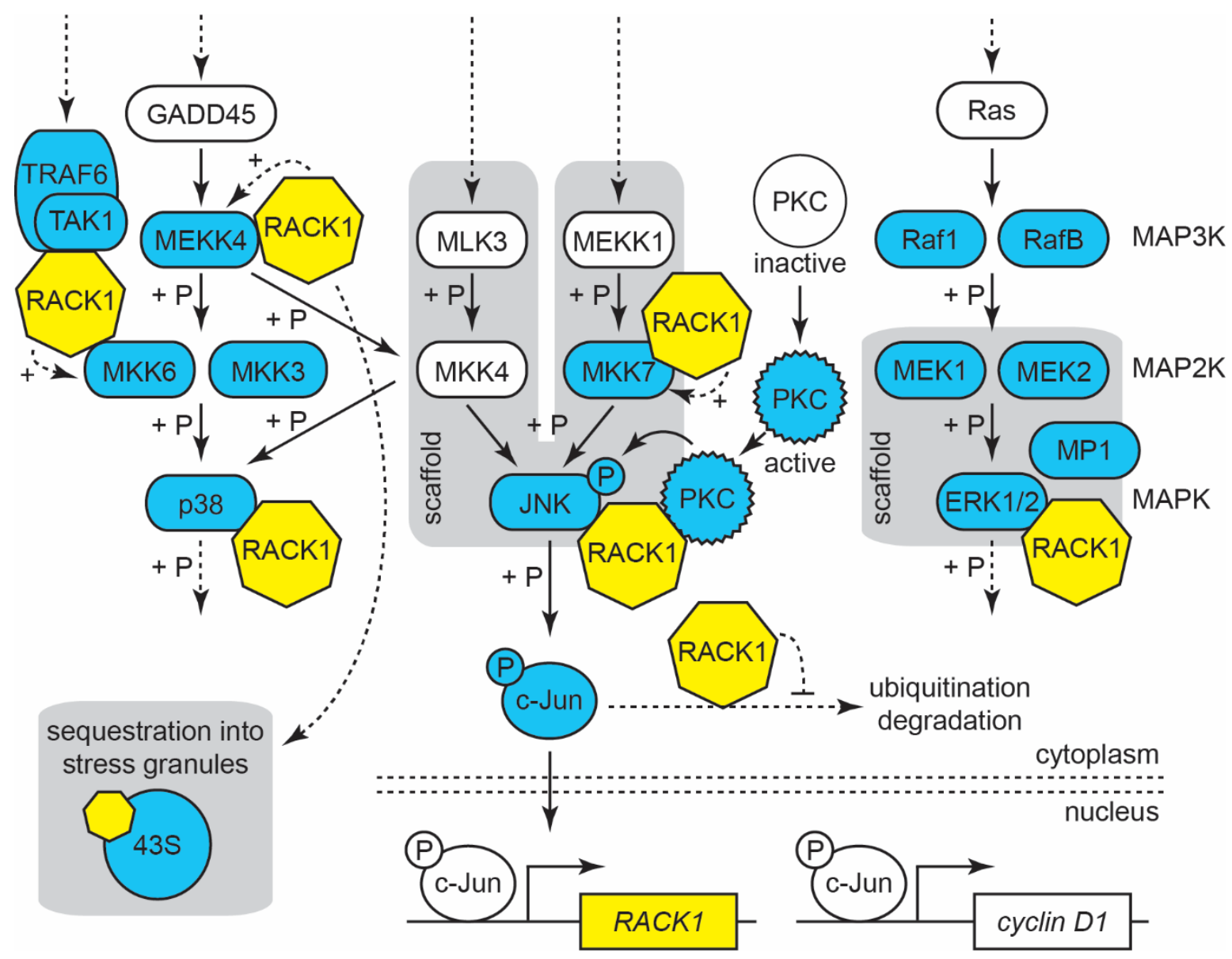

Figure 6. Model on the involvement of RACK1 in MAPK cascades in metazoans.

The model illustrates the interaction of RACK1 with components of different MAPK cascades and related processes. Proteins that have been reported to interact directly or are in a multisubunit complex with RACK1 are colored in blue. Dashed lines indicate up-stream or downstream signal transduction or regulatory processes that are not illustrated in more detail. References for interactions are as follows: MEKK4 (Arimoto et al., 2008), MKK7 (Guo et al., 2013), TRAF6 and TAK1 (Lin et al., 2015), MKK6 (Lin et al., 2015; Wang et al., 2015b), MKK3 (Wang et al., 2015b), JNK and activated PKC (López-Bergami et al., 2005), components of ERK1/2 pathway (Vomastek et al., 2007). The components of the ERK pathway were shown to interact with RACK1 in a complex. The illustrated interaction between ERK and RACK1 is representative for the whole pathway and might occur indirectly (Vomastek et al., 2007). RACK1 also promotes activity of MKK3 that is not illustrated in the figure due to space limitations. The inhibitory effect of RACK1 on the degradation of N-terminally phosphorylated c-Jun was reported by Zhang et al. (2012) and is described in detail in the following chapter 1.4.3. Localization of RACK1 to stress granules was shown by Arimoto et al. (2008). The overview of MAPK pathways is adapted from the KEGG database, and the model for RACK1's involvement in these pathways is partially adapted from Gandin et al. (2013b).

stimulated osteoclast formation. Accordingly, reduction of RACK1 levels reduces phosphorylation of p38 as well as RANKL-stimulated osteoclastogenesis (Lin et al., 2015).

Furthermore, ectopic expression of RACK1 in mouse L929 fibroblastoma cells suppresses tumor necrosis factor $\underline{\alpha}(\mathrm{TNF} \alpha)$-induced cell death most likely due to increased activation of p38 (Wang et al., 2015b). An interaction between RACK1 and the upstream kinases MKK3 and MKK6 of p38 was shown in L929 and human 293T cells. Upon TNFa stimulation, 
enhanced binding of RACK1 activates the kinases leading to increased phosphorylation of p38 (Wang et al., 2015b).

Using the Cercopithecus aethiops origin-defective $\underline{S} V-40$ (COS7) cell line derived from African green monkey kidney cells as well as HEK293 cells, RACK1 was further shown to interact with the MAP3K MEKK4/MTK1 that phosphorylates the upstream MAP2Ks MKK3 and MKK6 of p38 (Figure 6, Arimoto et al., 2008). In the absence of stress, RACK1 seems to tether at least two MEKK4 proteins together in an inactive state, thereby facilitating their activation as soon as the cells become exposed to certain stress conditions (Arimoto et al., 2008). MEKK4 additionally phosphorylates the MAP2K MKK4 that targets p38 as well but also the MAPK JNK.

The JNK signal transduction pathway is further affected by RACK1 through its direct interaction with the MAPK (López-Bergami et al., 2005). In HEK293T cells, PKC becomes activated upon treatment with UV irradiation or phorbol esters and is localized to JNK by RACK1 (Figure 6). PKC phosphorylates the MAPK at S129 and thereby augments the activation of JNK through its upstream MAP2Ks MKK4 and MKK7 (López-Bergami et al., 2005). Thus, RACK1 functions as a node to link PKC with MAPK signal transduction.

RACK1 was further described to directly interact with the upstream MAP2K MKK7 of JNK in human hepatocellular carcinoma (HCC) and HEK293T cells (Guo et al., 2013). The interaction between RACK1 and MKK7 promotes the interaction of MKK7 with its upstream MAP3Ks increasing its activity and leading to elevated JNK phosphorylation (Figure 6). Expression of RACK1 is elevated in clinical HCC tissue and could promote tumor growth through its interaction with MKK7 (Guo et al., 2013).

In the mouse fibroblast cell line NIH 3T3, RACK1 was shown to associate with central components of the MAPK/extracellular signal-regulated kinase (ERK) pathway: The MAP3Ks RafB and Raf1, the MAP2Ks MEK1 and MEK2, the MAPKs ERK1/2, and the scaffolding protein MP1 (Figure 6, Vomastek et al., 2007). The ERK pathway responses to extracellular substances, such as growth factors and hormones, and regulates cell proliferation and differentiation (Rubinfeld and Seger, 2005). RACK1 seems to link the pathway to integrin- and FAK-activation and is required for the localization of ERK to focal adhesions, where it promotes cell motility through the disassembly of these structures (Vomastek et al., 2007).

The afore mentioned experiments performed by Arimoto and colleagues (2008) revealed interaction between RACK1 and the MAP3K MEKK4, but neither confirmed the interaction of RACK1 with the MAP2Ks MKK3, MKK6, and MKK7, nor with the MAPKs JNK, ERK, 
and p38. The occasionally inconsistent observations for RACK1's interaction with components of MAPK cascades could be either due to a context-specific interaction of RACK1 with other proteins that varies between different model organisms or cell lines or it might be caused by differences in the experimental design. Interaction between RACK1 and p38b, for instance, was shown for D. melanogaster and was specific for a kinase-dead mutant of the MAPK (Belozerov et al., 2014).

\subsubsection{RACK1 as a central hub linking signal transduction with translation}

Most studies on RACK1's function in signal transduction pathways do not take the ribosomeassociation of the protein into consideration. Yet, as described before (see also chapter 1.4.1.2), RACK1 was identified as an anchoring protein for the kinases PKC $\beta I I$ and JNK at the translational machinery (Ceci et al., 2003; Ruan et al., 2012; Gandin et al., 2013a). Furthermore, activation of the MAP3K MEKK4 is regulated through sequestration of RACK1 into stress granules as part of stalled pre-initiation complexes (Figure 6, Arimoto et al., 2008). Certain stress conditions, such as hypoxia, induce granule formation and consequently reduce the amount of cytoplasmic RACK1 that is available for interaction with MEKK4 leading to decreased basal activity of the kinase and inhibition of its activation (Arimoto et al., 2008). Thus, besides mediating crosstalk between different signal transduction pathways, RACK1 also links these pathways to the translational machinery.

\subsubsection{RACK1-dependent protein-degradation of its interaction partners}

Mammalian RACK1 regulates the degradation of certain interaction partners, such as the hypoxia-inducible factor $\underline{1}$ (HIF-1, Liu et al., 2007a). The transcription factor HIF-1 is a heterodimer consisting of an $\alpha$ - and $\beta$-subunit (Wang and Semenza, 1995). Under low oxygen conditions, HIF-1 induces the transcription of genes that encode proteins required for increased oxygen delivery and adaptation of the energy metabolism (Semenza et al., 1994; Iyer et al., 1998). Hypoxia can be caused by environmental conditions, such as increased altitude or by defects in blood supply to tissues. Furthermore, hypoxia plays an essential role during embryogenesis (Iyer et al., 1998), and it also occurs frequently within cancer tissues where it causes HIF-1-dependent changes in cell-proliferation, angiogenesis, and cellular metabolism (reviewed in Semenza, 2013).

An important mechanism for the regulation of HIF-1 is the degradation of its $\alpha$-subunit in response to oxygen concentrations. Under normoxia, prolyl-hydroxylases modify HIF-1 $\alpha$ and thereby generate a binding site for the von Hippel-Lindau protein/Elongin-B/C E3 ubiquitin 
ligase that ubiquitinates the transcription factor subunit and thus triggers its proteasomal degradation. This pathway depends on cellular oxygen-levels and the metabolic status of the cell because the prolyl-hydroxylases use oxygen as well as $\alpha$-ketoglutarate as substrates for the modification of HIF-1 $\alpha$ (Ivan et al., 2001; Jaakkola et al., 2001; Semenza, 2013).

The stability of HIF-1 $\alpha$ is also regulated by an oxygen-independent pathway that involves RACK1 (Figure 7, Liu et al., 2007a). The $\beta$-propeller protein competes with the heat shock protein HSP90 and the septin-family member SEPT9_v1 for binding to the transcription factor subunit. Whereas binding of HSP90 and SEPT9_v1 stabilizes HIF-1 $\alpha$, RACK1 can recruit the Elongin-B/C E3 ubiquitin ligase to HIF-1 $\alpha$ via direct interaction with Elongin-C and thereby promotes ubiquitination and degradation of the transcription factor subunit (Liu et al., 2007a; Amir et al., 2009). The formation of this complex most likely relies on the homodimerization of RACK1 because both HIF-1 $\alpha$ as well as the E3 ubiquitin ligase interact with the sixth WD40 repeat of the $\beta$-propeller protein and thus only the RACK1 dimer can bind both proteins simultaneously (Liu et al., 2007b). Homodimerization of RACK1 involves the fourth WD40 repeat of each monomer and seems to require phosphorylation of its amino
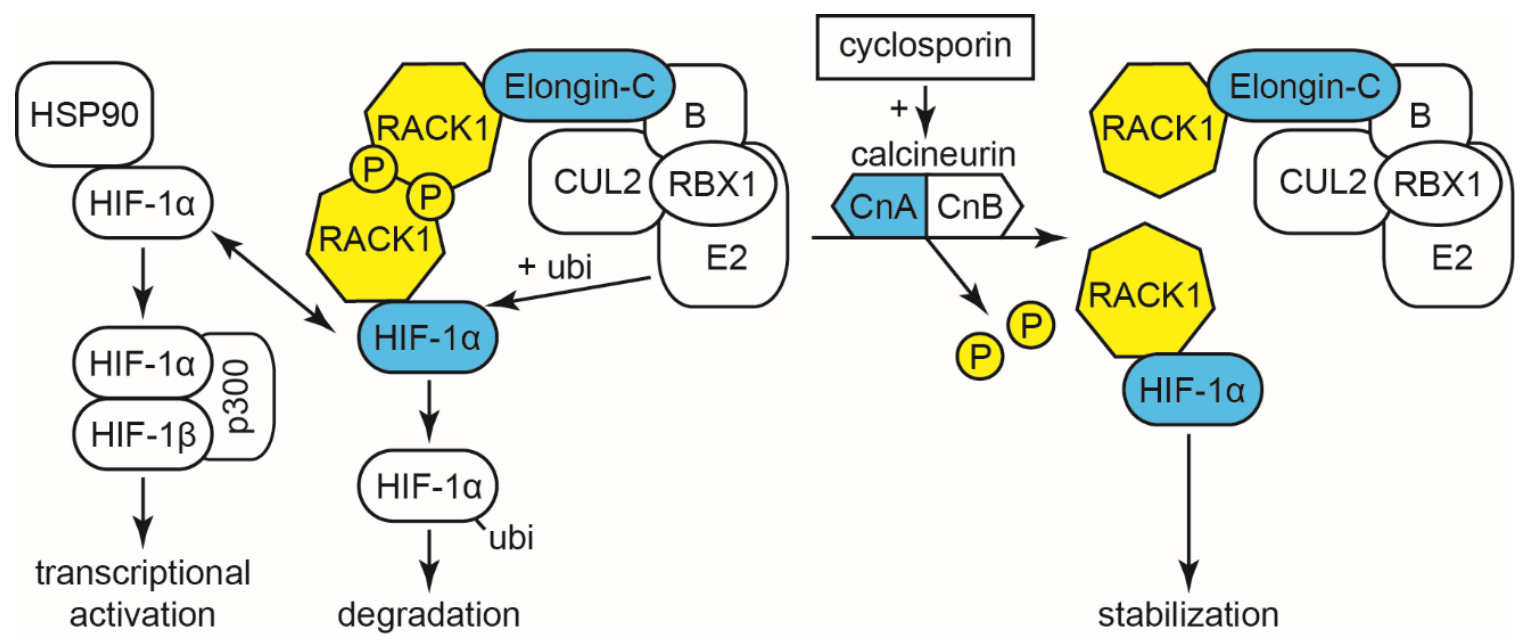

Figure 7. Model on RACK1-dependent and oxygen-independent regulation of HIF-1 $\alpha$ stability. RACK1 competes with HSP90 (or SEPT9_v1, not depicted here) for binding to the transcription factor subunit HIF-1 $\alpha$. HSP90-bound HIF- $1 \alpha$ is stabilized and can interact with the HIF-1 $\beta$-subunit and coactivators, such as $\mathrm{p} 300$, to induce transcription of target genes. RACK1-bound HIF-1 $\alpha$, however, is recruited to Elongin- $\mathrm{C}$ that is part of an E3 ubiquitin ligase complex further consisting of Elongin- $\underline{B}$ (B), Cullin- $\underline{2}$ (CUL2), ring box protein $\underline{1}$ (RBX1), and an E2 ubiquitin-conjugating enzyme (E2). HIF-1 $\alpha$ is ubiquitinated and degraded by the $26 \mathrm{~S}$ proteasome. Localization to the ligase complex is achieved through interaction of RACK1 with Elongin-C. Since both Elongin-C and HIF-1 $\alpha$ interact with the sixth WD40 repeat of RACK1, dimerization of the $\beta$-propeller protein is required. Dimerization involves the fourth WD40 repeat of each RACK1 monomer and seems to require phosphorylation at S146. RACK1 can interact with the catalytic subunit CnA of the phosphatase calcineurin $(\mathrm{CnB}$ is the regulatory subunit). Calcineurin can become activated by cyclosporine and subsequently dephosphorylates RACK1 leading to dissociation of the dimer and thus stabilization of HIF-1 $\alpha$. The figure is modified from Liu et al. (2007a and b). RACK1-interacting proteins are highlighted in blue. 
acid residue S146 within the fourth WD40 repeat. RACK1 dimer formation is impaired by activation of the calcium induced protein phosphatase $2 \mathrm{~B}$ (PP2B) that is also known as calcineurin. The interaction of RACK1 with the catalytic subunit of calcineurin seems to result in dephosphorylation of RACK1 at S146 leading to dissociation of the RACK1 homodimer into its subunits and thus stabilization of HIF-1 $\alpha$ (Figure 7, Liu et al., 2007b).

RACK1 further induces the degradation of the pro-apoptotic factor BimEL (Zhang et al., 2008). In the presence of the apoptosis inducing agent paclitaxel, RACK1 mediates the interaction of BimEL with an E3 ligase complex resulting in ubiquitination and proteasomedependent degradation of BimEL. Through this mechanism, RACK1 could protect cancer cells from apoptosis leading to tumor growth and drug resistance (Zhang et al., 2008).

RACK1 also affects the stability of the transcription factor c-Jun by regulating its interaction with Fbw7, the subunit for substrate recognition of an E3 ubiquitin ligase complex (Zhang et al., 2012a). The N-terminus of the oncoprotein c-Jun can be phosphorylated by the JNKs leading to increased transcriptional activity, DNA-binding, and stability of the protein (Papavassiliou et al., 1995; Musti et al., 1997; Weiss et al., 2003). RACK1 mediates interaction between N-terminally non-phosphorylated c-Jun and Fbw7 leading to ubiquitination and subsequent degradation of the transcription factor (Figure 6, Zhang et al., 2012a). N-terminally phosphorylated c-Jun, however, is excluded from this complex and thus stabilized. This further strengthens the role of the $\beta$-propeller protein in tumorigenesis (Zhang et al., 2012a).

In summary, RACK1 regulates degradation of HIF-1 $\alpha$, BimEL, and c-Jun through mediating their interaction with the respective ubiquitin E3 ligase complex. Furthermore, RACK1 induces ubiquitination and subsequent degradation of the pro-apoptotic protein Fem $1 \mathrm{~b}$ and the $\Delta$ Np63 transcription factor (Fomenkov et al., 2004; Subauste et al., 2009). Thus, RACK1 is an important regulator in post-translational regulation of protein expression for specific target proteins.

\subsection{Regulation of Asc1p/RACK1 through post-translational modifications}

Asc1p and its orthologues from higher eukaryotes interact with a plethora of different proteins. The Saccharomyces Genome ㅁatabase (SGD, http://www.yeastgenome.org) currently lists 85 proteins as physical interaction partners of Asc1p. Some of these interactions might occur indirectly via the formation of multi-protein complexes, but others were shown to involve direct protein-protein interaction, such as the binding of the eIF3csubunit Nip1p to Asc1p (Kouba et al., 2012). The Nip1p interaction site lies within WD 
repeats 1 to 3 of Asc1p (Kouba et al., 2012). Based on the crystal structure of the yeast 80S ribosome, the sites within Asc1p that interact with the ribosomal proteins Rps3, Rps16, and Rps17, (which are not included in the SGD list of interaction partners), can be precisely mapped (Figure 2, Ben-Shem et al., 2011). The interaction surface of the Asc1p homodimer is formed by blades 3 and 4 of each Asc1p monomer according to the crystal structure of the recombinantly expressed protein (Yatime et al., 2011). For all other direct physical interaction partners of Asc1p, the binding site has not been confined so far.

In contrast, the binding sites of many metazoan RACK1 interaction partners have been mapped to more specific regions within the $\beta$-propeller protein. Figure 8 illustrates that many RACK1 interaction partners share identical or overlapping binding sites. Many of these interactions might not interfere with each other because they are, for example, organism-, tissue-, or cell line-specific. However, considering all the additional interaction partners that are not included in Figure 8, the RACK1 interaction network demands multiple regulatory mechanisms, such as the post-translational modification of RACK1 itself. The previous chapters of this work already provided examples for a regulation of mammalian RACK1 through phosphorylation: The interaction of RACK1 with the focal adhesion kinase becomes stabilized through phosphorylation at Y52 by the c-Abl kinase (Kiely et al., 2009). Phosphorylation of mammalian RACK1 at Y246 by the Src kinase increases the interaction of RACK1 and Src and leads to inhibition of the kinase activity (Chang et al., 2001; Chang et al., 2002). Phosphorylation of RACK1 at Y246 further regulates its interaction with the mRNAbinding protein ZBP1 within transport RNPs in neurons, thereby modulating the translation of $\beta$-actin mRNA (Ceci et al., 2012). Moreover, RACK1 interacts with the MAPK p38b of D. melanogaster and was shown to be phosphorylated by this kinase at serine and/or threonine residues in vitro (Belozerov et al., 2014). In mammals, RACK1 is phosphorylated by the AMP-activated protein kinase at T50 during the onset of autophagy. This modification enhances the binding of RACK1 to components of the Atg14L-Beclin 1-Vps34-Vps15 complex and consequently promotes the formation of the autophagy initiation complex (Zhao et al., 2015).

Although the $\beta$-propeller structure is described as a rigid scaffold for protein-protein interactions, the Asc1/RACK1 proteins of S. cerevisiae, H. sapiens, and A. thaliana were shown to harbor flexible regions (Tarnowski et al., 2014). Thus, phosphorylation of Asc1p/RACK1 might contribute to define the shape of the $\beta$-propeller. RACK1 was reported to form homodimers in dependence on its phosphorylation at S146 (Liu et al., 2007b), and according to the crystal structure of the S. cerevisiae homodimer this interaction requires 


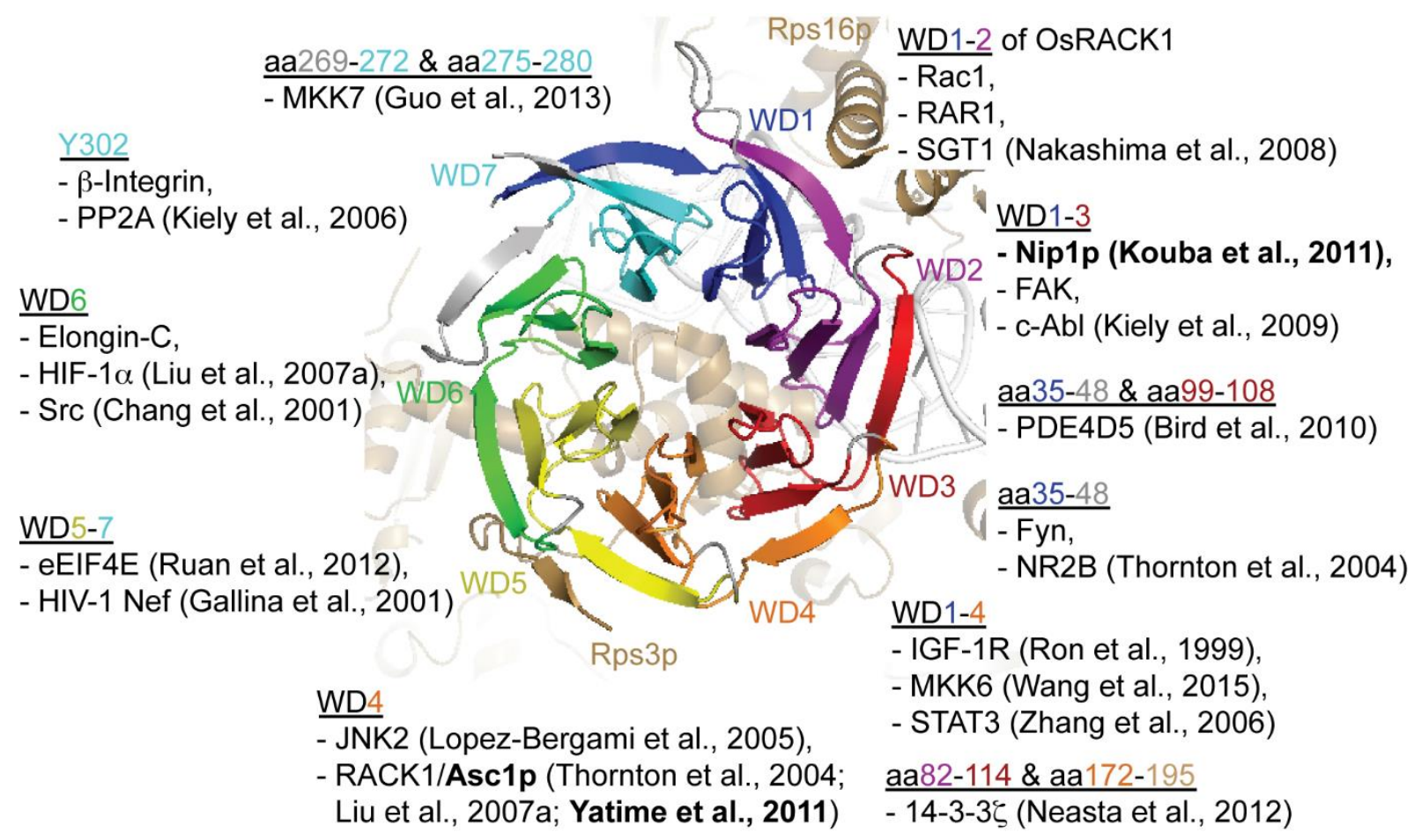

Figure 8. RACK1 interaction map.

The crystal structure of human RACK1 is shown bound to the 40S subunit of the ribosome. The WD40 repeats are differentially colored, and the remaining parts of RACK1 depicted in gray. The surrounding text indicates the localization of RACK1 binding sites for the named interactions partners. Besides interaction partners of metazoan RACK1, also O. sativa (Os) RACK1 and S. cerevisiae Asc1p binding partners (printed in bold) are included. The depiction of human RACK1 at the ribosome was modeled using the PyMOL Molecular Graphics System software on the basis of the PDB files with the IDs 4AOW (Ruiz Carrillo et al., 2012) and 4V88 (Ben-Shem et al., 2011) through merging the structure of human RACK1 on the position of S. cerevisiae Asc1p at the ribosome.

drastic rearrangement of the fourth blade of each monomer that might be indeed regulated through phosphorylation in this region (Figure 1D, Yatime et al., 2011). Phosphorylation of RACK1 might further regulate the turn-over of the protein as it was shown for A. thaliana RACK1A, which seems to be destabilized through phosphorylation by the with no lysine kinase $\underline{8}$ (WNK8, Urano et al., 2015).

So far, there exists no knowledge about a regulation of $S$. cerevisiae Asc1p through posttranslational modifications, although high-throughput studies identified several amino acid residues as targets for modifications. These modifications comprise phosphorylation (Chi et al., 2007; Smolka et al., 2007; Gnad et al., 2009; Holt et al., 2009), acetylation (Henriksen et al., 2012), succinylation (Weinert et al., 2013), and ubiquitination (Swaney et al., 2013). The high degree of conservation of Asc1p on the level of the amino acid sequence might also apply to some of its post-translational modifications. Thus, the analysis of post-translational modifications of $S$. cerevisiae Asc1p might reveal conserved features for the regulation of this protein. 


\subsection{Aim of this study}

The exposed position of the Asc1p $\beta$-propeller at the head of the 40S ribosomal subunit and its association with components of signal transduction pathways suggest a function of Asc1p as an interface between the translation machinery and signal transduction. To understand the function of Asc1p in cellular signaling in S. cerevisiae, the Asc1p-dependent phosphoproteome was analyzed. Since mammalian RACK1 has been reported to recruit kinases to the ribosome for the phosphorylation of translation initiation factors, a special interest was on proteins that contain Asc1p-sensitive phosphorylation sites and are associated to translational or co-translational processes. Due to its known association with components of phosphorylation cascades, Asc1p itself might be a target of regulatory phosphorylation. Here, phosphorylation sites of Asc1p should be identified, and their impact on Asc1p's function should be characterized through their targeted mutation to phosphorylation- or dephosphorylation-mimicking residues. Phenotypic characterization of the resulting mutant strains should reveal their importance for the functional integrity of Asc1p. Phosphorylation of Asc1p might support signal transduction to the translational machinery to adjust it to cellular needs. This might result in translational regulation of specific transcripts. Here, mRNAs were sought for that show Asc1p-dependent translation efficiency. Altogether, the results aim to contribute to characterize the function of the ribosomal protein Asc1 in signal transduction. 


\section{Materials and Methods}

For centrifugation of samples in $1.5 \mathrm{ml}$ or $2 \mathrm{ml}$ reaction tubes, the Hereaus Instruments Biofuge Pico or Hereaus Fresco 17 centrifuge (Thermo Scientific, Waltham, Massachusetts, USA) was used, and for centrifugation of samples in $15 \mathrm{ml}$ or $50 \mathrm{ml}$ tubes the eppendorf Centrifuge $5804 R$ (Eppendorf Wesseling-Berzdorf, Germany), the Sigma Laboratory Centrifuge 4K15C (Sigma Laborzentrifugen, Osterode am Harz, Germany) or the Hettich Rotanta/RP centrifuge (Hettich Lab Technology, Tuttlingen, Germany) was used. For picture editing, Adobe Photoshop Elements 5.0/CS4/CS6 and Adobe Illustrator CS4/CS6 (Adobe Systems, San Jose, California, USA) were applied, and for text and data processing Microsoft Word and Excel 2007/2010 (Microsoft, Redmond, Washington, USA) were used.

\subsection{S. cerevisiae strains}

The $S$. cerevisiae strains used in this study are of the $\Sigma 1278 \mathrm{~b}$ background (except for the BY4741-derived strain Y02724) and are listed in Table 1. For the generation of the asc1SNR24 ( $\left.\mathrm{sscl}^{-}\right)$strain that expresses the snoRNA U24, but not Asc1p, a loxP::URA3::loxP cassette was integrated in exon 1 of ASC1 resulting in strain RH3514. The marker was rescued through transient transformation with plasmid-borne Cre-recombinase (pSH63). This resulted in strain RH3510 with a loxP site in the ASC1 ORF that abrogates the translation of the mRNA (Rachfall et al., 2013). An ASCl deletion strain (RH3500) was generated through replacement of the ASC1 gene by a loxP::URA3::loxP cassette amplified from plasmid pUG72 according to Gueldener et al. (2002). This strain was used for subsequent construction of strains with the ASC1 cDNA (RH3502) or with ASC1 alleles carrying different codon-exchanges within the open-reading frame at the ASC1 locus (RH3529-RH3551， RH3574-RH3598， RH3611-RH3616， RH3623-RH3631， RH3635RH3644). The transformation cassettes contained the ASC1 cDNA or the mutated ascl-alleles carrying flanking regions identical to those of the ASCl gene for homologues recombination. Transformants were selected for their resistance against 5-fluoroorotic acid $(0.5 \mathrm{mg} / \mathrm{ml}$; \#R0812, Thermo Fisher Scientific, Waltham, Massachusetts, USA) due to the loss of the $U R A 3$ marker gene. Successful transformations were verified by polymerase chain reaction (PCR, chapter 2.5.1), Southern blot (chapter 2.7), and sequencing of the ASC1 locus (chapter 2.5.5). Arginine or arginine and lysine auxotrophic strains RH3487-RH3494 and RH3570RH3573 derived from strains RH2817 (ASCl), RH3263 ( $\triangle$ ascl), and RH3549 (asc1DE), respectively. The ARG4 and LYS1 genes were also replaced with the recyclable loxP::URA3::loxP marker according to Gueldener et al. (2002). As already described, strains 
were transiently transformed with plasmid-borne Cre-recombinase (pSH63) for marker rescue. An arginine and lysine auxotrophic ascl- strain was generated from the ASCl $\Delta$ arg4::loxP $\Delta$ lys $1:: \operatorname{lox} P$ strain $\mathrm{RH} 3493$ as already described: Integration of a loxP::URA3::loxP cassette in exon 1 of ASC1 resulted in strain RH3519. The subsequent rescue of the URA3-marker led to strain RH3520 with a loxP site in exon 1 of ASC1. For metabolic labeling with isotopically-labeled arginine and lysine, $\Delta \arg 4: \because$ loxP $\Delta$ lys $1::$ lox $P$ strains RH3493 (ASC1), RH3520 (asc 1 ), RH3573 (asc1DE), and RH3494 (Aascl) were used. Strains RH3431 (ASC1), RH3504 ( ascl $^{-}$), and RH3599 (asclDE) expressing C-terminally 3xmyc-tagged Flo8p were constructed by transformation of RH2817, RH3510, and RH3549 according to Janke et al. (2004). HBN1 and $Y H B 1$ deletion strains were generated as described for the ARG4 and LYS1 deletion strains using the loxP::kanMX::loxP cassette that was amplified from plasmid pUG6 according to Gueldener et al. (2002).

Table 1. S. cerevisiae strains used in this study.

For Asc1p phospho-site mutations that caused Asc1p-dependent phenotypes at least two independent clones with individual strain designations were generated and tested. The only exception is the asc ${ }^{\mathrm{T} 12 \mathrm{~A}} \mathrm{DE}$ strain.

\begin{tabular}{|c|c|c|}
\hline Strains & Genotype & Reference \\
\hline RH2817 & 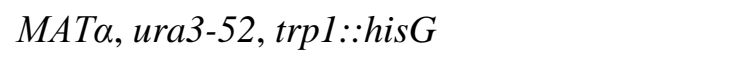 & Valerius et al., 2007 \\
\hline RH3263 & 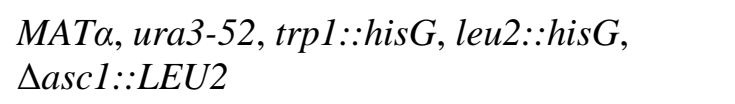 & Valerius et al., 2007 \\
\hline Y02724 & $\begin{array}{l}\text { BY4741, Mat a, his } 3 \Delta 1, \Delta l e u 2, \Delta m e t 15, \\
\Delta u r a 3, Y L R 113 w(\text { HOG1)::kanMX4 }\end{array}$ & Euroscarf collection \\
\hline RH3461 & 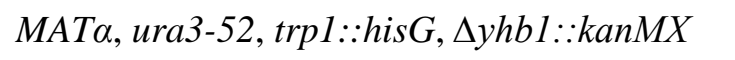 & Rachfall et al., 2013 \\
\hline RH3462 & 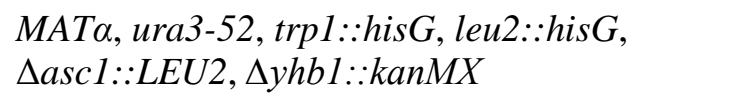 & Rachfall et al., 2013 \\
\hline RH3463 & 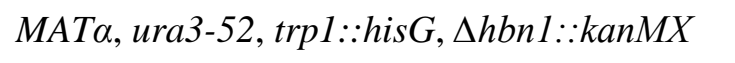 & Rachfall et al., 2013 \\
\hline RH3464 & 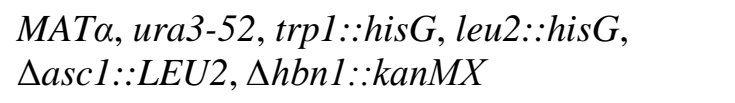 & Rachfall et al., 2013 \\
\hline RH3497 & 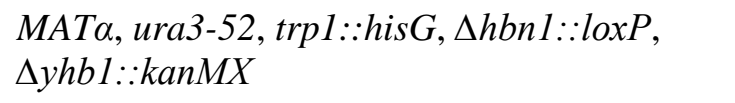 & Rachfall et al., 2013 \\
\hline RH3498 & 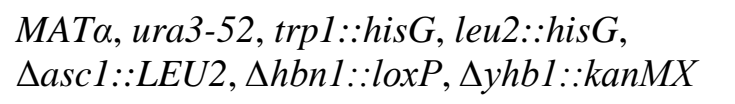 & Rachfall et al., 2013 \\
\hline RH3514 & $\begin{array}{l}\text { MATa, ura3-52, trp1::hisG, } \\
\text { asc1-URA3 SNR24 }\end{array}$ & $\begin{array}{l}\text { Nadine Smolinski } \\
\text { (Master thesis, MT) }\end{array}$ \\
\hline RH3510 & MATa, ura3-52, trp1::hisG, asc1-loxP SNR24 & Rachfall et al., 2013 \\
\hline RH3500 & 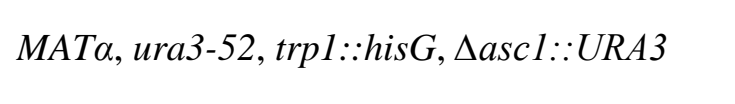 & $\begin{array}{l}\text { Sabrina Sander } \\
\quad \text { (MT) }\end{array}$ \\
\hline RH3502 & 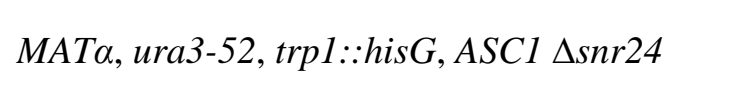 & $\begin{array}{l}\text { Sabrina Sander } \\
\text { (MT) }\end{array}$ \\
\hline RH3549, RH3550 & MATa, ura3-52, trp1::hisG, asc1DE & This work \\
\hline
\end{tabular}


Table 1. continued 1.

\begin{tabular}{|c|c|c|}
\hline Strains & Genotype & Reference \\
\hline RH3431 & MAT $\alpha$, ura3-52, trp $1:: h i s G$, FLO $8-$ myc $^{3}-T R P 1$ & Rachfall et al., 2013 \\
\hline RH3504 & 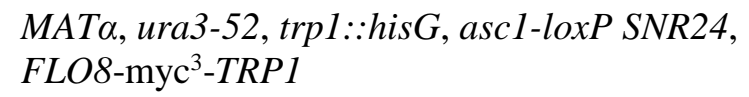 & Rachfall et al., 2013 \\
\hline RH3599 & $\begin{array}{l}\text { MAT } \alpha, \text { ura3-52, trp1::hisG, asc1DE, } \\
\text { FLO8-myc }{ }^{3}-T R P 1\end{array}$ & This work \\
\hline RH3623, RH3624, RH3625 & 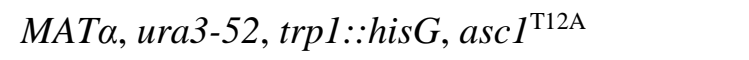 & This work \\
\hline RH3626 & 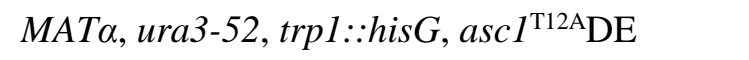 & This work \\
\hline RH3627, RH3628, RH3629 & 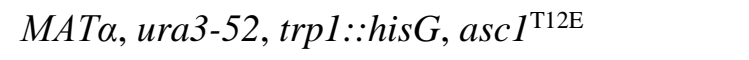 & This work \\
\hline RH3630, RH3631 & 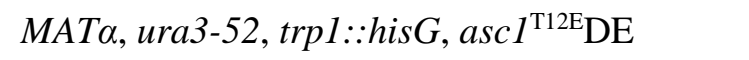 & This work \\
\hline RH3529 & 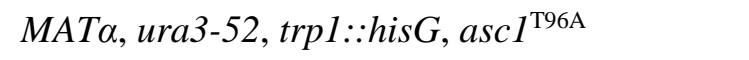 & This work \\
\hline RH3539, RH3540, RH3541 & MATa, ura3-52, $\operatorname{trp} 1: \because h i s G$, asc $1^{\mathrm{T} 96 \mathrm{~A}} \mathrm{DE}$ & This work \\
\hline RH3530 & MAT $\alpha$, ura3-52, trpl::hisG, asc1 $1^{\mathrm{T} 96 \mathrm{E}}$ & This work \\
\hline RH3542, RH3543 & 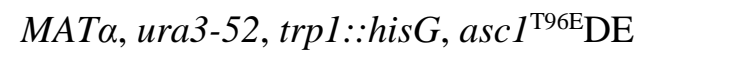 & This work \\
\hline RH3531 & 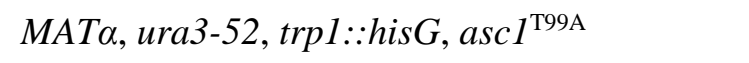 & This work \\
\hline RH3611, RH3612, RH3613 & 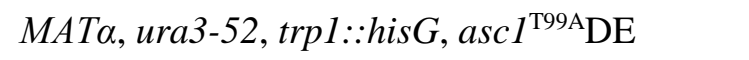 & This work \\
\hline RH3532 & MAT $\alpha$, ura3-52, trpl::hisG, asc1 $1^{\mathrm{T} 99 \mathrm{E}}$ & This work \\
\hline RH3614, RH3615, RH3616 & 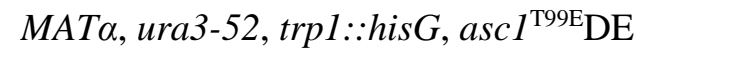 & This work \\
\hline RH3533, RH3534, RH3535 & 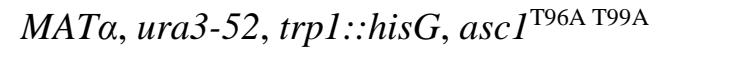 & This work \\
\hline RH3544, RH3545, RH3546 & 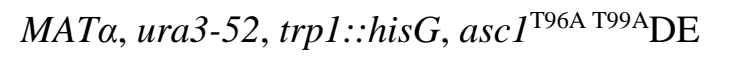 & This work \\
\hline RH3537, RH3538 & MATa, ura3-52, trpl::hisG, ascl $1^{\mathrm{T} 96 \mathrm{E} \mathrm{T} 99 \mathrm{E}}$ & This work \\
\hline RH3547, RH3548 & 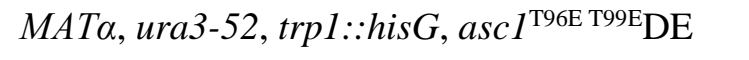 & This work \\
\hline RH3551 & MAT $\alpha$, ura3-52, trp $1: \because h i s G$, asc $1^{\mathrm{S} 120 \mathrm{~A}}$ & This work \\
\hline RH3575, RH3576, RH3577 & MATa, ura3-52, trp1::hisG, asc $1^{\mathrm{S} 120 \mathrm{~A}} \mathrm{DE}$ & This work \\
\hline RH3574 & MAT $\alpha$, ura3-52, trp $1:: h i s G$, asc $^{\mathrm{S} 120 \mathrm{E}}$ & This work \\
\hline RH3578, RH3579, RH3580 & MAT $\alpha$, ura3-52, $\operatorname{trp} 1:: h i s G$, asc $1^{\mathrm{T} 143 \mathrm{~A}}$ & This work \\
\hline RH3584, RH3585, RH3586 & MATo, ura3-52, trp1::hisG, asc1 ${ }^{\mathrm{T} 143 \mathrm{~A}} \mathrm{DE}$ & This work \\
\hline RH3581, RH3582, RH3583 & 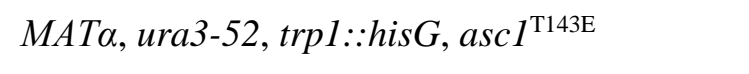 & This work \\
\hline RH3587, RH3588, RH3589 & MATa, ura3-52, trp1 ::hisG, asc1 ${ }^{\mathrm{T} 143 \mathrm{E}} \mathrm{DE}$ & This work \\
\hline RH3590 & 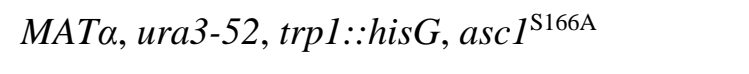 & This work \\
\hline RH3638 & MATa, ura3-52, trp1::hisG, asc1 ${ }^{\mathrm{S} 166 \mathrm{~A}} \mathrm{DE}$ & This work \\
\hline RH3591, RH3592 & 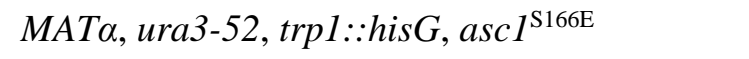 & This work \\
\hline RH3593, RH3594, RH3595 & 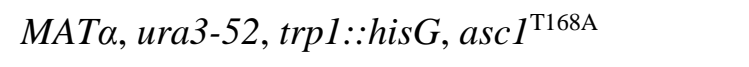 & This work \\
\hline RH3639, RH3640 & 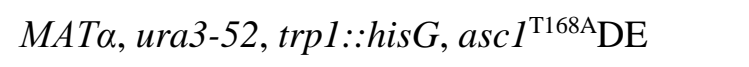 & This work \\
\hline RH3596, RH3597 & 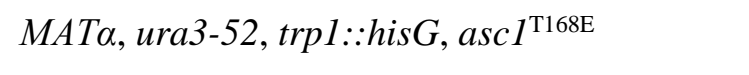 & This work \\
\hline RH3598 & MAT $\alpha$, ura3-52, $\operatorname{trpl}: \because$ hisG, asc1 $1^{\text {S166A T168A }}$ & This work \\
\hline RH3536 & MATo, ura3-52, trp1::hisG, asc ${ }^{\mathrm{S} 166 \mathrm{~A} \mathrm{~T} 168 \mathrm{~A}} \mathrm{DE}$ & This work \\
\hline
\end{tabular}


Table 1. continued 2.

\begin{tabular}{|c|c|c|}
\hline Strains & Genotype & Reference \\
\hline RH3637 & MAT $\alpha$, ura3-52, $\operatorname{trp} 1:: h i s G$, asc $1^{\mathrm{S} 166 \mathrm{E} \mathrm{T} 168 \mathrm{E}}$ & This work \\
\hline RH3635, RH3641, RH3642 & 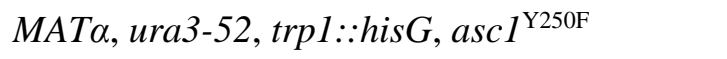 & This work \\
\hline RH3636, RH3643, RH3644 & MAT $\alpha$, ura3-52, trp1::hisG, asc1 ${ }^{\mathrm{Y} 250 \mathrm{~F}} \mathrm{DE}$ & This work \\
\hline RH3487 & 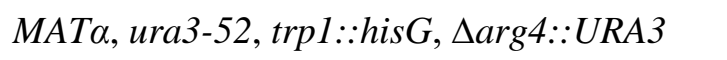 & This work \\
\hline RH3488 & 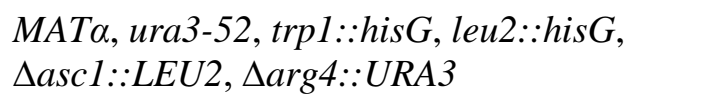 & This work \\
\hline RH3489 & 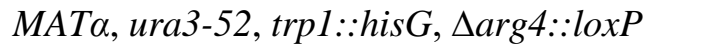 & This work \\
\hline RH3490 & 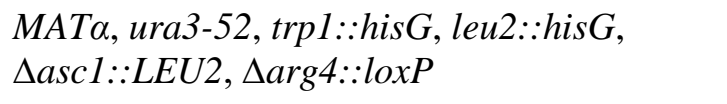 & This work \\
\hline RH3491 & 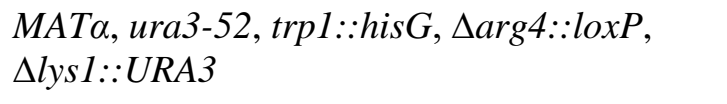 & This work \\
\hline RH3492 & 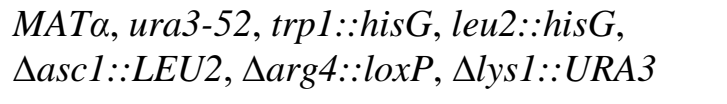 & This work \\
\hline RH3493 & 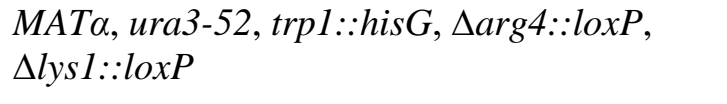 & This work \\
\hline RH3494 & 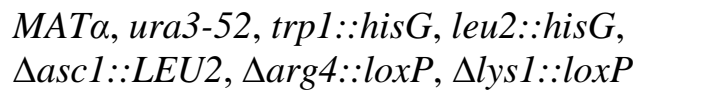 & This work \\
\hline RH3519 & 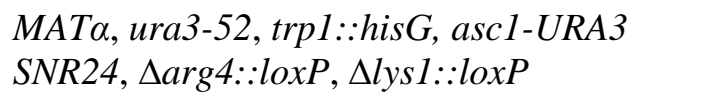 & This work \\
\hline RH3520 & 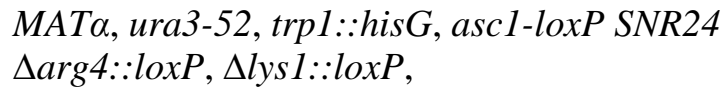 & This work \\
\hline RH3570 & 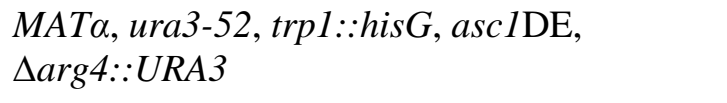 & This work \\
\hline RH3571 & $\begin{array}{l}\text { MAT } \alpha, \text { ura3-52, trpl } \because: h i s G, \text { asclDE, } \\
\text { Aarg4::loxP }\end{array}$ & This work \\
\hline RH3572 & 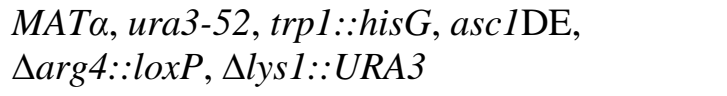 & This work \\
\hline RH3573 & $\begin{array}{l}\text { MAT } \alpha, \text { ura3-52, trpl } \because: \text { hisG, asc1DE, } \\
\Delta \arg 4: \because \operatorname{lox} P, \Delta \operatorname{lys} 1: \because \operatorname{lox} P\end{array}$ & This work \\
\hline
\end{tabular}

\subsection{Bacterial strain and plasmids}

The Escherichia coli strain DH5 $\alpha$ was used for preparation and amplification of plasmids (F',

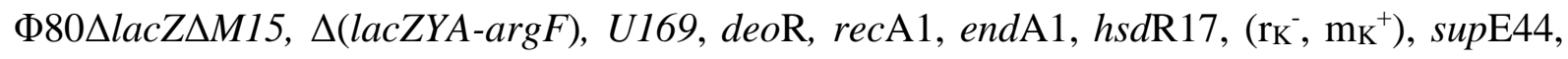
$\lambda$-, thi-1, gyrA96, relA1, Woodcock et al., 1989). Plasmids used in this study are listed in Table 2. Plasmid pME4132 was obtained through insertion of the ASC1 gene into the pASKIBA7plus plasmid (\#2-1406-000, IBA, Göttingen, Germany) according to the provided instructions. To generate plasmid pME4135, Strep-ASC1 was amplified from plasmid pME4132 introducing NheI and HindIII restriction sites for cloning into plasmid pME2835 (Nadine Smolinski, personal communication). Plasmid pME4041 was generated through 
Table 2. Plasmids used in this study.

\begin{tabular}{|c|c|c|}
\hline Plasmid & Description & Reference \\
\hline pUG6 & Amp ${ }^{R}$, pUCori, loxP ::kanMX::loxP & $\begin{array}{l}\text { Gueldener et al., } \\
\qquad 2002\end{array}$ \\
\hline pUG72 & Amp ${ }^{R}, p U$ Cori, loxP ::URA3::loxP & $\begin{array}{l}\text { Gueldener et al., } \\
2002\end{array}$ \\
\hline pSH63 & Amp ${ }^{R}$, pUCori, GAL1Prom, cre, TRP1, CEN/ARS & $\begin{array}{l}\text { Gueldener et al., } \\
\quad 2002\end{array}$ \\
\hline pME2791 & pRS416GAL1 with GAL1Prom, CYC1Term, URA3, CENIARS & Mumberg et al., 1994 \\
\hline pME2787 & pRS426MET25 with MET25Prom CYC1Term, URA3, $2 \mu \mathrm{m}$ & Mumberg et al., 1994 \\
\hline pME2624 & MET25Prom, CYC1Term, URA3, $2 \mu \mathrm{m}$, ASC1 & our collection \\
\hline pME2834 & MET25Prom, CYC1Term, URA3, $2 \mu \mathrm{m}$, ASC1-Strep & our collection \\
\hline pME2835 & MET25Prom, CYC1Term, URA3, $2 \mu \mathrm{m}$, Strep-ASC1 & our collection \\
\hline pME4041 & MET25Prom, CYC1Term, URA3, $2 \mu \mathrm{m}$, ASC1-cDNA-Strep & N. Smolinski (MT) \\
\hline $\begin{array}{l}\text { pASK- } \\
\text { IBA7plus }\end{array}$ & AmpR, fl origin, Strep-tag, Xa cleavage site & IBA \\
\hline pME4132 & AmpR, fl origin, Strep-Xa-ASC1 & N. Smolinski \\
\hline pME4135 & MET25Prom, CYC1Term, URA3, $2 \mu \mathrm{m}$, Strep-Xa-ASC1 & N. Smolinski \\
\hline pME4364 & pME2791 with $A S C 1$ and its native promoter (+500bp) & This work \\
\hline pME4384 & $\begin{array}{l}\text { pME } 2791 \text { with asc } 1 \mathrm{R} 38 \mathrm{DK} 40 \mathrm{E} \text { (asclDE) and the native } \\
\text { ASC1 promoter }\end{array}$ & This work \\
\hline pME4124 & pME2834 with asclDE & This work \\
\hline pME4365 & pME4364 with asc $1^{\mathrm{T} 12 \mathrm{~A}}$ & This work \\
\hline pME4366 & pME4364 with $a s c 1^{\mathrm{T} 12 \mathrm{~A}} \mathrm{DE}$ & This work \\
\hline pME4367 & pME4364 with asc $^{\mathrm{T} 12 \mathrm{E}}$ & This work \\
\hline pME4368 & pME4364 with asc1 ${ }^{\mathrm{T} 12 \mathrm{E}} \mathrm{DE}$ & This work \\
\hline pME4025 & pME2834 with asc $1^{\mathrm{T} 96 \mathrm{~A}}$ & N. Smolinski (MT) \\
\hline pME4370 & pME2834 with asc $^{\mathrm{T} 96 \mathrm{~A}} \mathrm{DE}$ & This work \\
\hline pME4026 & pME2834 with asc $^{\mathrm{T} 96 \mathrm{E}}$ & N. Smolinski (MT) \\
\hline pME4371 & pME2834 with $a s c 1^{\mathrm{T} 96 \mathrm{E}} \mathrm{DE}$ & This work \\
\hline pME4027 & pME2834 with asc $1^{\mathrm{T} 99 \mathrm{~A}}$ & N. Smolinski (MT) \\
\hline pME4372 & pME2834 with $\operatorname{asc} 1^{\mathrm{T} 99 \mathrm{~A}} \mathrm{DE}$ & This work \\
\hline pME4028 & pME2834 with ascl $1^{\mathrm{T} 99 \mathrm{E}}$ & N. Smolinski (MT) \\
\hline pME4373 & pME2834 with $a s c 1^{\mathrm{T} 99 \mathrm{E}} \mathrm{DE}$ & This work \\
\hline pME4029 & pME2834 with asc $1^{\text {T96A T99A }}$ & N. Smolinski (MT) \\
\hline pME4125 & pME2834 with asc $1^{\mathrm{T} 96 \mathrm{~A} \text { T99A } \mathrm{DE}}$ & This work \\
\hline pME4030 & pME2834 with asc $^{\text {T96E T99E }}$ & N. Smolinski (MT) \\
\hline pME4374 & pME2834 with asc $^{\text {T96E T99E }}{ }^{2 E}$ & This work \\
\hline pME4120 & pME2834 with asc $^{\mathrm{S} 120 \mathrm{~A}}$ & This work \\
\hline
\end{tabular}


Table 2. continued.

\begin{tabular}{|c|c|c|}
\hline Plasmid & Description & Reference \\
\hline pME4375 & pME4364 with asc ${ }^{\mathrm{S} 120 \mathrm{~A}} \mathrm{DE}$ & This work \\
\hline pME4121 & pME2834 with asc $^{\mathrm{S} 120 \mathrm{E}}$ & This work \\
\hline pME4122 & pME2834 with asc $1^{\mathrm{T} 143 \mathrm{~A}}$ & This work \\
\hline pME4376 & pME2834 with asc ${ }^{\mathrm{T} 143 \mathrm{~A}} \mathrm{DE}$ & This work \\
\hline pME4123 & pME2834 with $a s c 1^{\mathrm{T} 143 \mathrm{E}}$ & This work \\
\hline pME4377 & pME2834 with $a s c 1^{\mathrm{T} 143 \mathrm{E}} \mathrm{DE}$ & This work \\
\hline pME4031 & pME2834 with asc $1^{\mathrm{S} 166 \mathrm{~A}}$ & N. Smolinski (MT) \\
\hline pME4126 & pME2834 with $a s c 1^{\mathrm{S} 166 \mathrm{~A}} \mathrm{DE}$ & This work \\
\hline pME4032 & pME2834 with $a s c 1^{\mathrm{S} 166 \mathrm{E}}$ & N. Smolinski (MT) \\
\hline pME4033 & pME2834 with $a s c 1^{\mathrm{T} 168 \mathrm{~A}}$ & N. Smolinski (MT) \\
\hline pME4127 & pME2834 with ascl ${ }^{\mathrm{T} 168 \mathrm{~A}} \mathrm{DE}$ & This work \\
\hline pME4034 & pME2834 with $a s c 1^{\mathrm{T} 168 \mathrm{E}}$ & N. Smolinski (MT) \\
\hline pME4035 & pME2834 with $a s c 1^{\text {S166A T168A }}$ & N. Smolinski (MT) \\
\hline pME4128 & pME2834 with $a s c 1^{\mathrm{S} 166 \mathrm{~A} \text { T168A } \mathrm{DE}}$ & This work \\
\hline pME4036 & pME2834 with $a s c l^{\text {S166E T168E }}$ & N. Smolinski (MT) \\
\hline pME4378 & pME2834 with $a s c 1^{\mathrm{Y} 250 \mathrm{~F}}$ & This work \\
\hline pME4379 & pME2834 with asc ${ }^{\mathrm{Y} 250 \mathrm{~F}} \mathrm{DE}$ & This work \\
\hline
\end{tabular}

amplification of the ASC1 cDNA from a cDNA sample generated from total RNA of the $\Sigma 1278$ b strain background with primers introducing BamHI and HindIII restriction sites for cloning into pME2834. The QuantiTect Reverse Transcription Kit (\#205314, Qiagen, Hilden Germany) was used to generate cDNA from RNA according to the manufacturer's instructions. Plasmid pME4364 is derived from pME2791 and carries ASC1 under control of its native promoter (+500 bp upstream of ASC1 according to Kleinschmidt et al., 2006). ASC1 was amplified together with its promoter from genomic $S$. cerevisiae DNA using oligonucleotides that introduced SacI and HindIII restriction sites for subsequent cloning. Plasmids carrying ascl with codon exchanges were constructed via a two-step PCR strategy: In the first reaction, the codon exchange(s) was (or were) introduced within an oligonucleotide bearing the mutated codon(s) in its center using an ASC1 wild-type carrying plasmid (pME2834 or pME4364) as template. Codons for serine and threonine were exchanged for GCT (alanine) as well as for GAA (glutamate). The tyrosine 250 codon was substituted for TTT (phenylalanine), and R38 and K40 encoding triplets were exchanged for GAT (aspartate) and GAA (glutamate), respectively. In the second PCR, the complete asc 1 allele was amplified flanked by the respective restriction sites for cloning into the parent 
vector (BamHI and HindIII restriction sites for cloning into pME2834 and SacI and HindIII restriction sites for cloning into pME2791). For construction of plasmids pME4025pME4036, pME4120-pME4128, pME4370-pME4374, and pME4376-pME4379, plasmid pME2834 served as the parent vector. Plasmid pME4364 served as parent vector for plasmids pME4365-pME4368, pME4375, and pME4384. For plasmids comprising the R38D K40E codon exchanges in combination with a phosphorylation site mutation, either plasmid pME4124 or pME4384 was used as template for PCR. Alternatively, plasmids that already comprised a phosphorylation site mutation were used as template for PCR, and the R38D $\mathrm{K} 40 \mathrm{E}$ mutation was introduced by using oligonucleotides bearing the respective codon exchanges. The plasmids served as templates for amplification of transformation cassettes to integrate the mutated ascl alleles into the yeast genome at the original ASCl locus.

\subsection{Cultivation of microorganisms}

\subsubsection{Cultivation of $S$. cerevisiae}

Yeast strains were cultivated in liquid yeast nitrogen base medium (1.5 g/l YNB without amino acids and ammonium sulfate, $5 \mathrm{~g} / \mathrm{l}$ ammonium sulfate, $2 \%$ glucose) containing the respective supplements or in yeast extract peptone dextrose (YEPD) medium ( $2 \%$ peptone, $1 \%$ yeast extract, $2 \%$ glucose). Solid media contained $2 \%$ agar. Minimal with vitamins (MV) plates contained $1.45 \mathrm{~g} / \mathrm{l} \mathrm{YNB}, 5.52 \mathrm{~g} / \mathrm{l}$ ammonium sulfate, $10 \%$ succinic acid, $8.5 \mathrm{~g} / \mathrm{l} \mathrm{KOH}$, and their $\mathrm{pH}$ was adjusted to 7.2. If required, $20 \mathrm{mg} / \mathrm{l} \mathrm{L}$-arginine, $20 \mathrm{mg} / \mathrm{l} \mathrm{L}$-histidine, $30 \mathrm{mg} / \mathrm{l}$ L-isoleucine, $\quad 30 \mathrm{mg} / \mathrm{l} \quad$ L-lysine $\mathrm{HCl}, \quad 20 \mathrm{mg} / \mathrm{l} \quad$ L-methionine, $20 \mathrm{mg} / \mathrm{l}$ uracil, $20 \mathrm{mg} / \mathrm{l} \mathrm{L-tryptophan,} 30 \mathrm{mg} / \mathrm{l} \mathrm{L}-$ leucine, $150 \mathrm{mg} / \mathrm{l} \mathrm{L-valine.} \mathrm{Yeast} \mathrm{cells} \mathrm{were} \mathrm{grown} \mathrm{at} 30{ }^{\circ} \mathrm{C}$. Liquid cultures were grown on a shaker (100 to $120 \mathrm{rpm}$ ), and growth of the cells was monitored by measuring the optical density (OD) at $600 \mathrm{~nm}$. The inoculation volumes $\left(\mathrm{V}_{\mathrm{i}}\right.$ in $\mathrm{ml})$ for main cultures were calculated with the formula:

$$
\mathrm{V}_{\mathrm{i}}=\left(\mathrm{V}_{\mathrm{m}} \cdot O \mathrm{OD}_{\mathrm{m}} \cdot \mathrm{e}^{-\mu \cdot \Delta t}\right) / O D_{\mathrm{p}}
$$

$\left(\mathrm{V}_{\mathrm{m}}=\right.$ volume of main-culture in $\mathrm{ml}, \mathrm{OD}_{\mathrm{m}}=$ desired optical density of the main-culture after $\Delta \mathrm{t}, \mathrm{OD}_{\mathrm{p}}=\mathrm{OD}$ of pre-culture, $\mu=$ growth rate $(0.29 / \mathrm{h}$ for $\mathrm{YNB}), \Delta \mathrm{t}=$ growth time in $\left.\mathrm{h}\right)$.

For SILAC-based phospho-proteome and proteome analyses cultures were grown in liquid YNB medium supplemented with $100 \mathrm{mg} / \mathrm{l}$ differentially labeled L-arginine as well as L-lysine. Stable isotopically labeled amino acids were purchased from Silantes (München, Germany): ${ }^{13} \mathrm{C}_{6}$-L-arginine $\mathrm{HCl}$ (\#201203902), ${ }^{13} \mathrm{C}_{6}{ }^{15} \mathrm{~N}_{4}-\mathrm{L}$-arginine $\mathrm{HCl}$ (\#201603902), 4,4,5,5-D 4 -L-lysine $\mathrm{HCl}$ (\#211103912), and ${ }^{13} \mathrm{C}_{6}{ }^{15} \mathrm{~N}_{2}$-L-lysine $\mathrm{HCl}$ (\#211603902). Further experiment-specific growth conditions are described in the respective paragraphs. 


\subsubsection{Cultivation of $E$. coli}

E. coli cells were cultivated in liquid lysogeny broth (LB) medium (1\% bacto-trypton, $0.5 \%$ yeast extract, $1 \% \mathrm{NaCl}$ ) on a shaker at $37{ }^{\circ} \mathrm{C}$. The medium was supplemented with $100 \mu \mathrm{g} / \mathrm{ml}$ ampicillin for selective conditions. Solid media contained $2 \%$ agar.

\subsection{Isolation of DNA from microorganisms}

\subsubsection{Plasmid DNA purification from $E$. coli}

For isolation of plasmid DNA, E. coli cells of a $5 \mathrm{ml} \mathrm{LB}$ overnight culture were harvested by centrifugation, and plasmids were isolated using the QIAprep Spin Miniprep Kit (\#27106, Qiagen) according to the manufacturer's instructions. Plasmid DNA was eluted with $50 \mu 1$ $\mathrm{H}_{2} \mathrm{O}$. For PCR, samples were diluted 1:50.

\subsubsection{Isolation of DNA from $S$. cerevisiae cells}

Cells were grown overnight in $10 \mathrm{ml}$ YEPD medium to saturation and harvested by centrifugation for isolation of DNA according to Hoffman and Winston (1987). The pellet was washed in $0.5 \mathrm{ml} \mathrm{H} \mathrm{H}_{2} \mathrm{O}$ and transferred to a $1.5 \mathrm{ml}$ reaction tube. After spinning down the cells, the supernatant was discarded, and the cells were disrupted in the presence of $0.2 \mathrm{ml}$ lysis buffer (2\% Triton X-100, $1 \%$ SDS, $100 \mathrm{mM} \mathrm{NaCl}, 10 \mathrm{mM}$ Tris pH 8.0, 1 mM EDTA), $0.2 \mathrm{ml}$ phenol/chloroform/isoamylalcohol (25:24:1, \#A156.2, Carl Roth, Karlsruhe, Germany), and an equal volume of glass beads. Cells were disrupted through vigorous shaking for 3 to $4 \mathrm{~min}$. $0.2 \mathrm{ml} \mathrm{H}_{2} \mathrm{O}$ were added, and the samples were centrifuged for $5 \mathrm{~min}$ at $13,000 \mathrm{rpm}$. The upper aqueous layer was transferred into a new tube, and $0.2 \mathrm{ml}$ phenol/ chloroform/isoamylalcohol were added. The samples were mixed and centrifuged again at 13,000 rpm for $5 \mathrm{~min}$. The upper phase was transferred into a new tube, and DNA was precipitated by adding $1 \mathrm{ml}$ ethanol followed by incubation at $-20{ }^{\circ} \mathrm{C}$ for several minutes or overnight. Samples were centrifuged for $5 \mathrm{~min}$ at 13,000 rpm followed by removal of the supernatant. Pellets were dissolved in $0.4 \mathrm{ml} \mathrm{H}_{2} \mathrm{O}$, and $3 \mu \mathrm{l} \mathrm{RNase} \mathrm{A}(10 \mathrm{mg} / \mathrm{ml})$ was added. After 5 to $10 \mathrm{~min}$ incubation at $37^{\circ} \mathrm{C}, 10 \mu \mathrm{l} 4 \mathrm{M}$ ammonium acetate and $1 \mathrm{ml}$ ethanol were added, and samples were stored again at $-20^{\circ} \mathrm{C}$ to precipitate the DNA. After centrifugation at 13,000 rpm for $5 \mathrm{~min}$, the supernatant was discarded, pellets were dried and then dissolved in 40 to $50 \mu 1 \mathrm{H}_{2} \mathrm{O}$. For PCR, samples were diluted 1:50. 


\subsection{Cloning techniques}

\subsubsection{Polymerase chain reaction}

DNA was amplified with the polymerase chain reaction (PCR; Saiki et al., 1985). Depending on the subsequent application of the amplified PCR product, different thermostable polymerases were used. For amplification of DNA for site-specific homologous recombination and plasmid construction, Phusion High-Fidelity DNA polymerase (\#F-530L, Thermo Scientific), Pfu DNA polymerase (\#EP0572, Thermo Scientific), or KOD DNA polymerase (\#71085-3, Merck, Darmstadt, Germany) were used. For analytic PCRs, Taq DNA polymerase (\#EP0404, Thermo Scientific) was applied. $10 \mathrm{mM}$ dNTP stocks (prepared from dATP \#0141, dCTP \#R0151, dGTP \#R0161, dTTP \#0171, Thermo Scientific) were used accept for the $K O D$ polymerase that was provided together with a $2 \mathrm{mM}$ dNTP stock (molarities apply to each dNTP). All polymerases were used according to the manufacturer's instructions. Primers for PCR were ordered from Eurofins MWG Operon (Ebersberg, Germany).

\subsubsection{Restriction digestion of DNA}

DNA was digested with type II restriction endonucleases for plasmid construction or prior to Southern blot analysis. Restriction enzymes and their respective buffers (Thermo Scientific or New England Biolabs, Frankfurt am Main, Germany) were used according to the manufacturer's instructions in a sample volume of $20 \mu 1$. Samples were incubated for at least $1.5 \mathrm{~h}$ at $30^{\circ} \mathrm{C}$ or $37^{\circ} \mathrm{C}$ depending on the applied enzyme.

\subsubsection{Agarose gel electrophoresis}

DNA samples were mixed with 0.1 volumes of 10x loading dye (10\% Ficoll 400, $200 \mathrm{mM}$ EDTA, $\mathrm{pH} 8.0,0.2 \%$ bromphenol blue sodium salt, $0.2 \%$ xylene cyanol) and applied on horizontal $1 \%$ agarose gels containing $0.5 \mu \mathrm{g} / \mathrm{ml}$ ethidium bromide. Agarose gels were prepared in TAE buffer ( $40 \mathrm{mM}$ Tris base, $20 \mathrm{mM}$ acetic acid, $1 \mathrm{mM}$ EDTA) that was also used as running buffer for separation of the DNA fragments through electrophoresis at $90 \mathrm{~V}$ (Voytas, 2001). For visualization of DNA, the Gel iX2O Imager (Intas Science Imaging Instruments, Göttingen, Germany) was used. If applicable, DNA bands were excised from agarose gels under UV light, and DNA was extracted using the QIAquick Gel Extraction Kit (\#28706, Qiagen) according to the manufacturer's instructions. 


\subsubsection{Ligation of DNA fragments}

For ligation of linear DNA fragments, $1 \mu 1$ T4 DNA ligase (\#EL0016, Thermo Scientific), $3 \mu 1$ of the supplied 10x ligation buffer (Thermo Scientific), 50 to $100 \mathrm{ng}$ digested plasmid DNA, and a 2- to 5-fold excess of insert DNA (or $\mathrm{H}_{2} \mathrm{O}$ for controls) were mixed and filled up to $30 \mu \mathrm{l}$ with $\mathrm{H}_{2} \mathrm{O}$. The sample was incubated for at least $10 \mathrm{~min}$ at room temperature and subsequently used for transformation of E. coli cells.

\subsubsection{DNA sequencing}

$100 \mathrm{ng}$ of DNA and $5 \mathrm{pmol}$ of the appropriate primer in a volume of $5 \mu \mathrm{l}$ were submitted to the Göttingen Genomics Laboratory (G2L, Göttingen, Germany) for sequencing. Alternatively, sequencing of DNA was carried out by the SEQLAB Sequencing Laboratories Göttingen GmbH (Göttingen, Germany): $18 \mathrm{ng}$ per 100 bp of template DNA was used for linear DNA fragments and 720 to $1200 \mathrm{ng}$ for sequencing of plasmids in a final volume of $12 \mu \mathrm{l} .3 \mu \mathrm{l}$ of the primer $(10 \mathrm{pmol} / \mu \mathrm{l})$ were added.

\subsection{Transformation procedures}

\subsubsection{Transformation of $E$. coli}

Preparation of competent E. coli cells was performed according to Inoue et al. (1990). For the preparation of competent $E$. coli cells, $250 \mathrm{ml}$ super optimal broth (SOB) medium (2\% tryptone, $0.5 \%$ yeast extract, $10 \mathrm{mM} \mathrm{NaCl}, 2.5 \mathrm{mM} \mathrm{KCl}, 10 \mathrm{mM} \mathrm{MgCl} 2,10 \mathrm{mM} \mathrm{MgSO}$ ) were inoculated with DH5 $\alpha$ cells and incubated at $20{ }^{\circ} \mathrm{C}$ at $200 \mathrm{rpm}$ for at least $24 \mathrm{~h}$ until an $\mathrm{OD}_{600}$ of 0.6 was reached. Cells were placed on ice for $10 \mathrm{~min}$ and harvested by centrifugation at $2,500 \mathrm{xg}$ for $10 \mathrm{~min}$ at $4{ }^{\circ} \mathrm{C}$. The pellet was resuspended in $80 \mathrm{ml}$ transformation buffer (TB, $10 \mathrm{mM}$ HEPES, $15 \mathrm{mM} \mathrm{CaCl}_{2}, 250 \mathrm{mM} \mathrm{KCl}, \mathrm{pH} 6.7,55 \mathrm{mM}$ $\mathrm{MnCl}_{2}$ ). Cells were stored on ice for $10 \mathrm{~min}$ and centrifuged at $2,500 \mathrm{xg}$ for $10 \mathrm{~min}$ at $4{ }^{\circ} \mathrm{C}$. The pellet was gently resuspended in $20 \mathrm{ml}$ TB buffer. $1.4 \mathrm{ml}$ DMSO was added with swirling, and cells were incubated for $10 \mathrm{~min}$ on ice. Aliquots were prepared, frozen in liquid nitrogen, and stored at $-80{ }^{\circ} \mathrm{C}$. For the transformation of E. coli, competent cells were thawed on ice. Samples from the DNA ligation were each mixed with $200 \mu 1$ of these cells. After incubation on ice for $30 \mathrm{~min}$, samples were placed at $42{ }^{\circ} \mathrm{C}$ for $90 \mathrm{sec}$ and then placed back on ice for $3 \mathrm{~min}$. Each sample was mixed with $800 \mu \mathrm{LB}$ medium and incubated at $37{ }^{\circ} \mathrm{C}$ for $1 \mathrm{~h}$ on a shaker. After centrifugation at 5,000 rpm for $2 \mathrm{~min}$, the supernatant was discarded. Cells were resuspended in the remaining liquid and spread on solid selective media. Plates were incubated overnight at $37^{\circ} \mathrm{C}$. 


\subsubsection{Transformation of $S$. cerevisiae}

Transformations were performed using the lithium acetate method (Ito et al., 1983). 600 to $800 \mu \mathrm{l}$ of a $10 \mathrm{ml}$ YEPD pre-culture were transferred to $10 \mathrm{ml}$ fresh YEPD medium and incubated for additional 5 to $6 \mathrm{~h}$. Cells were harvested by centrifugation at 2,000 rpm for $3 \mathrm{~min}$. The cell pellet was resuspended in $10 \mathrm{ml}$ lithium acetate/TE buffer (100 mM lithium acetate, $10 \mathrm{mM}$ Tris-HCl, $1 \mathrm{mM}$ EDTA, pH 8.0). For genomic transformations using linear DNA fragments, cells were incubated for 15 to $20 \mathrm{~min}$ on a shaker. For transformation of plasmid DNA, cells were only briefly incubated. Samples were centrifuged again at 2,000 rpm for $3 \mathrm{~min}$. The supernatant was discarded, and cells were resuspended in the remaining liquid. The volume was filled up to $400 \mu 1$, and the sample was divided into two $200 \mu \mathrm{l}$ aliquots. To one of the samples the linear DNA of the transformation cassette or the plasmid DNA was added, while the second one served as negative control. $20 \mu \mathrm{l}$ of prewarmed $\left(65^{\circ} \mathrm{C}\right.$ ) carrier DNA (salmon sperm DNA, $10 \mathrm{mg} / \mathrm{ml}$ in TE buffer, $\mathrm{pH}$ 8.0) were added to both samples. For the transformation of the same strain with more than one linear DNA fragment or plasmid, all volumes were adjusted accordingly. Samples were mixed with $800 \mu 150 \%$ PEG 4000 (prepared in lithium acetate/TE buffer) and incubated at $30{ }^{\circ} \mathrm{C}$ for $30 \mathrm{~min}$ followed by a heat shock at $42{ }^{\circ} \mathrm{C}$ for $25 \mathrm{~min}$. Samples were centrifuged at 7,000 rpm for $30 \mathrm{sec}$, and the supernatant was removed. Cells were resuspended in $1 \mathrm{ml}$ YEPD medium and incubated for $1 \mathrm{~h}$ (transformation of plasmid DNA) or $3 \mathrm{~h}$ (chromosomal integration) at $30{ }^{\circ} \mathrm{C}$ on a shaker. Cells were harvested by centrifugation at 4,000 rpm for 10 to $20 \mathrm{sec}$. Most of the supernatant was discarded. Cells were resuspended in the remaining liquid, spread onto solid selective media, and incubated for at least $3 \mathrm{~d}$ until colonies formed.

\subsection{Southern blot analysis}

Integration of DNA fragments at the correct locus in the genome of S. cerevisiae was confirmed by Southern analysis according to Southern (1975). Chromosomal DNA was digested overnight with the respective restriction enzymes (Thermo Scientific). Samples were heated at $65{ }^{\circ} \mathrm{C}$ for $10 \mathrm{~min}$, mixed with loading dye, and applied onto $1 \%$ agarose gels. DNA fragments were separated according to their size at $70 \mathrm{~V}$ for $10 \mathrm{~min}$ followed by $90 \mathrm{~V}$ for $90 \mathrm{~min}$. Gels were then washed with the first washing buffer $(0.25 \mathrm{M} \mathrm{HCl})$ for $10 \mathrm{~min}$, with the second $(0.5 \mathrm{M} \mathrm{NaOH}, 1.5 \mathrm{M} \mathrm{NaCl})$ for $25 \mathrm{~min}$, and with the third $(1.5 \mathrm{M} \mathrm{NaCl}, 0.5 \mathrm{M}$ Tris, $\mathrm{pH}$ 7.5) for $30 \mathrm{~min}$. Afterwards, the DNA was transferred onto a nylon membrane (Hybond-N Membrane, \#RPN203N, GE Healthcare, München, Germany) by capillary blotting for at least $3 \mathrm{~h}$. The membrane was then dried at $75^{\circ} \mathrm{C}$ for $7 \mathrm{~min}$, and the DNA was 
cross-linked to the membrane through $3 \mathrm{~min}$ exposure of each side of the membrane to UV light. The membrane was incubated at $55{ }^{\circ} \mathrm{C}$ for $30 \mathrm{~min}$ in prewarmed hybridization buffer (0.5 M NaCl, 4\% Blocking Reagent, \#NIP552, GE Healthcare), and the labeled probe was added for hybridization overnight. Probes were labeled using the AlkPhos Direct Labeling Reagents (\#RPN3680, GE Healthcare) according to the manufacturer's instructions. The membranes were washed twice with the first washing buffer ( $2 \mathrm{M}$ urea, $0.1 \%$ SDS, $50 \mathrm{mM}$ sodium phosphate $\mathrm{pH} 7.0,150 \mathrm{mM} \mathrm{NaCl}, 1 \mathrm{mM} \mathrm{MgCl}_{2}, 0.2 \%$ Blocking Reagent) at $55^{\circ} \mathrm{C}$ for $10 \mathrm{~min}$ and twice with the second washing buffer (50 mM Tris base, $100 \mathrm{mM} \mathrm{NaCl}$, $2 \mathrm{mM} \mathrm{MgCl} 2, \mathrm{pH} \mathrm{10}$ ) at room temperature for $5 \mathrm{~min}$. For the chemiluminescence reaction, the membrane was incubated with $1 \mathrm{ml}$ of the CDP-Star Detection Reagent (\#RPN3682, GE Healthcare). For detection of chemiluminescence, membranes were exposed to the Amersham Hyperfilm $^{T M}-E C L^{T M}$ (GE Healthcare) followed by development of the film.

\subsection{Protein analyses}

\subsubsection{Preparation of whole-cell protein extracts from $S$. cerevisiae}

This paragraph describes preparation of protein extracts for Western blot analyses as well as proteome analyses. In case that the protocol differs for other applications, the changes are specified in the respective paragraphs. In general, S. cerevisiae cultures were grown in $50 \mathrm{ml}$ YNB medium to midlog phase $\left(\mathrm{OD}_{600}=0.8,40 \mathrm{ml}\right.$ cultures for proteome analysis $)$ and harvested by centrifugation at 3,000 rpm for $4 \mathrm{~min}$ at $4{ }^{\circ} \mathrm{C}$. Cells were washed with ice-cold breaking buffer (100 mM Tris-HCl pH 7.5, $200 \mathrm{mM} \mathrm{NaCl,} \mathrm{20 \%} \mathrm{glycerol,} 5 \mathrm{mM}$ EDTA) and lysed in $500 \mu 1$ breaking buffer supplemented with $0.5 \% \beta$-mercaptoethanol, 1 complete $^{\mathrm{TM}}$ protease inhibitor tablet (\#11836145001, Roche Diagnostics, Mannheim, Germany) per $50 \mathrm{ml}, 1$ PhosSTOP ${ }^{\mathrm{TM}}$ phosphatase inhibitor cocktail tablet (\#04906837001, Roche Diagnostics) per $10 \mathrm{ml}, 1 \mathrm{mM} \mathrm{NaF}, 8 \mathrm{mM} \beta$-glycerol phosphate, and $0.5 \mathrm{mM}$ sodium vanadate through vigorous shaking (frequency $30 \mathrm{sec}^{-1}, 4 \mathrm{~min}$ ) with an equal volume of glass beads ( $\varnothing 0.25$ to $0.50 \mathrm{~mm}$ ) using the mixer mill Retsch MM400 (Retsch, Haan, Germany). The samples were incubated in the presence of $4 \%$ SDS at $65{ }^{\circ} \mathrm{C}$ for $10 \mathrm{~min}$. Samples were subjected to centrifugation at $13,000 \mathrm{rpm}$ for $15 \mathrm{~min}$ at room temperature, and the supernatant was collected as protein extract. Protein concentrations were determined using the BCA reagent (\#23224 and \#23228, Thermo Fisher Scientific) according to the manufacturer's instructions. 


\subsubsection{Purification of Strep-tagged Asc1p}

For identification of Asc1p phospho-sites, strain RH3263 ( $\operatorname{ascl}$ ) was transformed with plasmid pME2834 (ASC1-Strep) or pME4135 (Strep-Xa-ASC1) and cultivated in 1 to 101 liquid YNB medium to an $\mathrm{OD}_{600}$ of 0.8. Harvested cells were washed with ice-cold breaking buffer (10 mM HEPES pH 7.9, $10 \mathrm{mM} \mathrm{KCl,} 1.5 \mathrm{mM} \mathrm{MgCl}_{2}$ ) and lysed in breaking buffer (approximately 5 times the volume of the cell pellet) supplemented with $0.5 \mathrm{mM}$ PMSF, $0.5 \mathrm{mM}$ DTT, and protease and phosphatases inhibitors (as described before) through vigorous shaking with glass beads. Samples were centrifuged at 13,000 rpm for $15 \mathrm{~min}$ at $4{ }^{\circ} \mathrm{C}$, and the supernatant was applied on Strep-Tactin ${ }^{\circledR}$ sepharose $^{\circledR}$ columns for affinity purification of Strep-tagged Asc1p (Strep-tag ${ }^{\circledR}$ Starter Kit, \#2-1101-000, IBA). The purification was performed at $4{ }^{\circ} \mathrm{C}$ following the instructions of the manufacturer's protocol. Eluate fractions were subjected to SDS-polyacrylamide gel electrophoresis (SDS-PAGE, chapter 2.8.3).

For identification of Asc1p and Asc1DEp interaction partners, strain RH3494 ( $\triangle a s c 1$ $\Delta$ arg4 $\Delta l y s$ l) was transformed with plasmids pME2834 (ASC1-Strep), pME4124 (asc1DEStrep), and pME2624 (ASC1), respectively. The resulting three different strains were cultivated in $150 \mathrm{ml}$ YNB medium supplemented with differentially labeled amino acids to an $\mathrm{OD}_{600}$ of 0.8 . Cell pellets were washed with the wash buffer $(100 \mathrm{mM}$ Tris- $\mathrm{HCl}, \mathrm{pH} 8.0$, $150 \mathrm{mM} \mathrm{NaCl}, 1 \mathrm{mM}$ EDTA) of the Strep-tag ${ }^{\circledR}$ Starter Kit, harvested by centrifugation and resuspended in $500 \mu \mathrm{l}$ of the same buffer supplemented with $0.5 \mathrm{mM}$ PMSF, 0.5 mM DTT, and protease and phosphatase inhibitors (as described before). Cells were lysed through vigorous shaking with glass beads. Samples were centrifuged for $8 \mathrm{~min}$ at $13,000 \mathrm{rpm}$ at $4{ }^{\circ} \mathrm{C}$. The supernatant was transferred into a new tube, and centrifugation was repeated. The three supernatants were mixed and directly applied onto a Strep-Tactin ${ }^{\circledR}$ Spin Column (\#2-1850010, IBA). $20 \mu \mathrm{l}$ of the pooled protein extracts were retained for SDS-PAGE. The columns were used for protein purification according to the manufacturer's instructions. Proteins were eluted from the column with $50 \mu$ l elution buffer $(100 \mathrm{mM}$ Tris- $\mathrm{HCl}, \mathrm{pH} 8.0,150 \mathrm{mM} \mathrm{NaCl}$, $1 \mathrm{mM}$ EDTA, $2 \mathrm{mM}$ D-biotin). $5 \mu \mathrm{l}$ of the total protein extract sample and one half of the eluate fraction were subjected to SDS-PAGE.

\subsubsection{SDS-polyacrylamide gel electrophoresis}

Protein samples were mixed in a 2:1 ratio with $3 \mathrm{x}$ loading dye $(0.25 \mathrm{M}$ Tris $-\mathrm{HCl} \mathrm{pH} 6.8$, $30 \%$ glycerol, $15 \% \beta$-mercaptoethanol, $7 \%$ SDS, $0.3 \%$ bromphenol blue) and heated at $65{ }^{\circ} \mathrm{C}$ for $10 \mathrm{~min}$ before they were subjected to SDS-polyacrylamide gel electrophoresis (SDS- 
PAGE) according to Laemmli (1970). Samples were applied onto 12\% SDS-polyacrylamide gels that were placed in electrophoresis buffer $(25 \mathrm{mM}$ Tris base, $250 \mathrm{mM}$ glycine, $0.1 \%$ SDS). SDS-gels consisted of a lower running gel (375 mM Tris $\mathrm{pH} 8.8,12 \%$ acrylamide/bisacrylamide 37.5:1 (Rotiphorese ${ }^{\circledR}$ Gel 30, \#3029.1, Carl Roth), 2 mM EDTA, $0.1 \% \mathrm{SDS})$ and an upper stacking gel (125 mM Tris $\mathrm{pH} 6.8,5 \%$ acrylamide/bisacrylamide 37.5:1, $2 \mathrm{mM}$ EDTA, 0.1\% SDS). Proteins were separated electrophoretically for $10 \mathrm{~min}$ at $100 \mathrm{~V}$ and subsequently at $150-200 \mathrm{~V}$ until the separation of proteins was sufficient. This was most often the case when the bromphenol blue left the gel. The PageRuler ${ }^{T M}$ Prestained Protein Ladder (\#26616, Thermo Fisher Scientific) was used to estimate the sizes of proteins.

\subsubsection{Western blot analysis}

Western blot experiments were performed according to Burnette (1981). Proteins were transferred from SDS-gels onto nitrocellulose membranes (Amersham ${ }^{T M}$ Protran $^{\circledR}$ Western blotting membrane, \#GE10600002, Sigma-Aldrich, München, Germany) by electrophoretic blotting in a Mini-Trans-Blot-Electrophoretic-Cell (Bio-Rad Laboratories, München, Germany) filled with transfer buffer (25 mM Tris base, $190 \mathrm{mM}$ glycine, 0.02\% SDS, 20\% methanol) for $1.5 \mathrm{~h}$ at $100 \mathrm{~V}$. After blotting, the membranes were stained with Ponceau red ( $0.2 \%$ Ponceau S, 3\% trichloroacetic acid) for $5 \mathrm{~min}$, washed with water and photographed using the FUSION-SL-4 (Peqlab, Erlangen, Germany). Ponceau red staining was removed through washing with phosphate buffered saline (PBS, $8 \mathrm{mM} \mathrm{Na}_{2} \mathrm{HPO}_{4}, 2 \mathrm{mM} \mathrm{NaH} \mathrm{PO}_{4}$, $150 \mathrm{mM} \mathrm{NaCl}$ ) and subsequent incubation with the blocking buffer (PBS with 5\% milk powder) for at least $1 \mathrm{~h}$ at room temperature or overnight at $4{ }^{\circ} \mathrm{C}$. Membranes were incubated for $2 \mathrm{~h}$ at room temperature or overnight at $4{ }^{\circ} \mathrm{C}$ with the primary antibody diluted in the blocking buffer. The membranes were incubated with monoclonal mouse anti-c-myc (dilution: 1:2,000, \#2276, Cell Signaling Technology, Danvers, Massachusetts, USA), polyclonal goat anti-Rap1p (1:500, \#yN-18, Santa Cruz Biotechnology, Heidelberg, Germany), polyclonal rabbit anti-Tec1p (1:1,000, Hans-Ulrich Mösch, Philipps-Universität, Marburg, Germany), polyclonal anti-Asc1p (1:1,000, Andrew Link, Vanderbilt University Medical Center, Nashville, USA), or polyclonal phospho-p38 MAPK (Thr180/Tyr182) (1:500, \#9211, Cell Signaling Technology) antibodies. The anit-Rap1p, anti-Tec1p, and antiAsc1p antibodies were diluted in PBS with $5 \%$ milk powder. Tris buffered saline (TBS, $150 \mathrm{mM}$ Tris, $150 \mathrm{mM} \mathrm{NaCl}, \mathrm{pH} 7.2-7.4$ ) with $5 \%$ milk powder and $0.1 \%$ Tween 20 was used for dilution of the anti-c-myc antibody. TBS with $5 \%$ bovine serum albumin (BSA) and $0.1 \%$ Tween 20 was used for blocking and preparation of the antibody dilution when the 
phosphorylation-specific antibody phospho-p38 MAPK (Thr180/Tyr182) was applied. Membranes were washed three times with PBS or TBS, respectively, followed by incubation with the peroxidase-coupled goat anti-mouse (1:5,000, \#115-035-003, Dianova, Hamburg, Germany), donkey anti-goat (1:2,000, \#sc-2020, Santa Cruz Biotechnology), or goat antirabbit (1:2,000, \#G21234, MoBiTec, Göttingen, Germany) secondary antibodies. The goat anti-rabbit antibody was diluted in TBS with 5\% BSA when it was used in combination with the phospho-p38 MAPK (Thr180/Tyr182) antibody. The membranes were washed as described before. For the peroxidase reaction, $100 \mu \mathrm{l} 2.5 \mathrm{mM}$ luminol, $44 \mu \mathrm{l} 40 \mu \mathrm{M}$ paracumaric acid, and $6.15 \mu \mathrm{l} 30 \% \mathrm{H}_{2} \mathrm{O}_{2}$ were added to $20 \mathrm{ml} 100 \mathrm{mM}$ Tris $\mathrm{pH} 8.5$ and applied onto the membrane followed by 1 min incubation on a shaker. Chemiluminescence was detected using the FUSION-SL-4. Signals were quantified relative to the Ponceau red staining as loading control according to Rivero-Gutiérrez et al. (2014) using the BiolD software Version 15.01 (Vilber Lourmat, Eberhardzell, Germany).

\subsubsection{Colloidal Coomassie staining of proteins}

Protein staining within SDS-gels was done using colloidal Coomassie G250 and was performed according to Kang et al. (2002). All steps of the procedure were performed with constant shaking of the SDS-gel in the respective solutions and at room temperature. After SDS-PAGE, the gel was incubated in fixing solution (40\% ethanol, $10 \%$ acetic acid) for at least $1 \mathrm{~h}$. The gel was washed twice for at least 10 min with $\mathrm{H}_{2} \mathrm{O}$ followed by staining with the Coomassie G250 solution (0.1\% Coomassie Brilliant Blue G250, 5\% aluminium sulfate(14-18)-hydrate, $10 \%$ methanol, $2 \%$ orthophosphoric acid) overnight. To prepare the staining solution, aluminium sulfate was first dissolved in $\mathrm{H}_{2} \mathrm{O}$ followed by the addition of methanol. Coomassie Brilliant Blue G250 was added and dissolved. Finally, phosphoric acid was added, and the solution was filled up with $\mathrm{H}_{2} \mathrm{O}$ to the final volume. After staining, the gel was washed with $\mathrm{H}_{2} \mathrm{O}$. Prior to in-gel trypsin digestion of proteins (chapter 2.8.6) the Coomassie staining was reduced through incubation of the gel or the gel pieces in fixing solution overnight. To remove the fixing solution, gels were washed with $\mathrm{H}_{2} \mathrm{O}$ at least twice for $10 \mathrm{~min}$.

\subsubsection{In-gel protein digestion with trypsin}

The in-gel digestion of proteins with trypsin was performed according to Shevchenko et al. (1996). Gel lanes were cut into pieces of approximately 1.5 to $2 \mathrm{~mm}^{2}$. The pieces were transferred into reaction tubes, covered with acetonitrile and shaken for $10 \mathrm{~min}$. Acetonitrile 
was removed, and the gel pieces were dried in the Savant SPDIIIV SpeedVac Concentrator (Thermo Scientific). $150 \mathrm{ml} 10 \mathrm{mM}$ DTT solution (in $100 \mathrm{mM} \mathrm{NH}_{4} \mathrm{HCO}_{3}$ ) were added to each sample followed by incubation at $56{ }^{\circ} \mathrm{C}$ for $1 \mathrm{~h}$. The DTT solution was removed, and $150 \mu 155 \mathrm{mM}$ iodoacetamide (prepared in $100 \mathrm{mM} \mathrm{NH}_{4} \mathrm{HCO}_{3}$ ) were added. After $45 \mathrm{~min}$ incubation at room temperature and in the dark, the solution was removed, and $150 \mu 1$ $100 \mathrm{mM} \mathrm{NH}_{4} \mathrm{HCO}_{3}$ were added. On a shaker, samples were incubated for $10 \mathrm{~min}$. The liquid was exchanged for $150 \mu \mathrm{l}$ acetonitrile, and the samples were incubated again for $10 \mathrm{~min}$. The washing steps with $100 \mathrm{mM} \mathrm{NH}_{4} \mathrm{HCO}_{3}$ and with acetonitrile were repeated once. Gel pieces were dried in the SpeedVac and subsequently covered with trypsin (\#37283.01, SERVA Electrophoresis, Heidelberg, Germany) digestion buffer, which was prepared according to the manufacturer's instructions. After $45 \mathrm{~min}$ incubation on ice, remaining trypsin digestion buffer was removed. The gel pieces were covered with $25 \mathrm{mM} \mathrm{NH}_{4} \mathrm{HCO}_{3}$, and samples were incubated at $37^{\circ} \mathrm{C}$ overnight. The supernatant was collected in a new reaction tube. Gel pieces were covered with $20 \mathrm{mM} \mathrm{NH}_{4} \mathrm{HCO}_{3}$ and shaken for at least $10 \mathrm{~min}$. The supernatant was collected again in the same tube, and the gel pieces were covered with 50\% acetonitrile $/ 5 \%$ formic acid. After shaking of the samples for at least $20 \mathrm{~min}$, the supernatant was collected. The last step was repeated twice. Prior to the collection of supernatants samples were always centrifuged for $1 \mathrm{~min}$ at 13,000 rpm. The peptide-containing samples were dried in the SpeedVac. Peptides were reconstituted in $20 \mu 1$ liquid chromatography-mass spectrometry (LC-MS) sample buffer (2\% acetonitrile, $0.1 \%$ formic acid) and directly subjected to LC-MS analysis (chapter 2.8.9) or purified using C18 stop and go extraction (stage) tips according to Rappsilber et al. (2003 and 2007). Two C18 disks were punched out from a Solid Phase Extraction Disk (\#2215, 3M, Neuss, Germany) and placed in a 100 to $200 \mu 1$ pipette tip. This stage tip was in turn placed into a reaction tube using an adaptor. The C18 material was equilibrated with $100 \mu 1$ methanol/0.1\% formic acid, followed by $100 \mu 1$ $70 \%$ acetonitrile $/ 0.1 \%$ formic acid, and twice $100 \mu 10.1 \%$ formic acid. For each step, the samples were centrifuged for $2 \mathrm{~min}$ at maximum speed. After loading of the peptide solution on the C18 material, the samples were centrifuged for $5 \mathrm{~min}$ at $4,000 \mathrm{rpm}$. Two times $100 \mu \mathrm{l}$ $0.1 \%$ formic acid were applied followed by 2 min centrifugation at 10,000 rpm for washing of the samples. Peptides were eluted from the C18 material with $60 \mu 170 \%$ acetonitrile/0.1\% formic acid. Samples were centrifuged for $5 \mathrm{~min}$ at 4,000 rpm. The eluted peptides were dried in the SpeedVac, reconstituted in $20 \mu \mathrm{l}$ LC-MS sample buffer, and subjected to LC-MS analysis. 


\subsubsection{In-solution digestion of proteins with $\mathrm{LysC}$ and trypsin}

Proteins were digested in-solution when high amounts of peptides were required for phosphopeptide enrichment. Protein samples were prepared as described in chapter 2.8.1 with the exceptions that cell extracts were subjected to sonication for subsequent filter-aided sample preparation (FASP, Wiśniewski et al., 2009). Protein concentrations were determined as described before (chapter 2.8.1). For the in-solution digest, $1 \mathrm{mg}$ protein of each sample was subjected to chloroform-methanol extraction to reduce the amount of SDS in the samples (Wessel and Flügge, 1984). For the chloroform-methanol extraction, $100 \mu$ l aliquots of the protein extract were prepared. If necessary, volumes were adjusted accordingly. Successively, each sample was mixed with $400 \mu \mathrm{l}$ methanol, followed by $100 \mu 1$ chloroform and $300 \mu 1$ $\mathrm{H}_{2} \mathrm{O}$. After each step, the samples were mixed through vigorous shaking for $1 \mathrm{~min}$. After centrifugation at $13,000 \mathrm{rpm}$ for $5 \mathrm{~min}$ at $4{ }^{\circ} \mathrm{C}$, the upper phase was discarded, and $300 \mu \mathrm{l}$ methanol were added. After vigorous shaking for $1 \mathrm{~min}$, the samples were centrifuged at 13,000 rpm for $10 \mathrm{~min}$ at $4{ }^{\circ} \mathrm{C}$, and afterwards the supernatant was discarded. The pellets were dried and then dissolved in $200 \mu \mathrm{l}$ buffer UA each (8 M urea prepared in $100 \mathrm{mM}$ Tris$\mathrm{HCl} \mathrm{pH}$ 8.5). Proteins were in-solution digested with LysC (\#125-05061, Wako Chemicals, Neuss, Germany) and trypsin (\#37283.01, SERVA Electrophoresis) according to the FASP protocol (Wiśniewski et al., 2009) using centrifugal filter units (\#MRCF0R030, Merck). The protein concentration was determined using the BCA reagent as described before and adjusted to $1.25 \mu \mathrm{g} / \mu \mathrm{l}$ with UA. For one phospho-proteome analysis, four filter units were each loaded with $200 \mu \mathrm{l}$ of the protein solution. All steps were performed at room temperature, and all centrifugation steps were carried out at 13,000 rpm for $15 \mathrm{~min}$ except when stated otherwise. If necessary, the flow-through was removed between the centrifugation steps. The filter units were centrifuged, refilled twice with $200 \mu \mathrm{l}$ UA and centrifuged again. $200 \mu \mathrm{l}$ UA with $3 \mu 11$ M DTT were added followed by gentle mixing. After 30 min incubation, the filter units were centrifuged, refilled with $200 \mu \mathrm{l}$ UA followed by another round of centrifugation. $100 \mu 150 \mathrm{mM}$ iodoacetamide prepared in 8 M UA were added, and the samples were gently mixed. After incubation for $20 \mathrm{~min}$ at room temperature, the filter units were centrifuged for $10 \mathrm{~min} .100 \mu \mathrm{l}$ buffer UB ( $8 \mathrm{M}$ urea prepared in $100 \mathrm{mM}$ Tris- $\mathrm{HCl} \mathrm{pH} 8.0$ ) were added followed by centrifugation. The step was repeated twice. Protein samples were mixed with $40 \mu \mathrm{l}$ UB containing LysC in an enzyme to protein ratio of 1:50. Filter units were sealed with parafilm and incubated overnight. The filter units were transferred into a new collection tube. Samples were mixed with $120 \mu \mathrm{l} 25 \mathrm{mM} \mathrm{NH}_{4} \mathrm{HCO}_{3}$ containing trypsin in an enzyme to protein ratio of 1:100. Samples were incubated for $4 \mathrm{~h}$ and then centrifuged for $15 \mathrm{~min}$. $50 \mu \mathrm{l}$ 
$0.5 \mathrm{M} \mathrm{NaCl}$ were added to the membrane of the filter unit, and the samples were centrifuged for 20 min. Peptide samples were acidified and desalted with C18 cartridges (\#98060401985, 3M) according to Wiśniewski et al. (2009). Each cartridge was placed into a $15 \mathrm{ml}$ tube, $1 \mathrm{ml}$ methanol was applied onto the cartridge followed by centrifugation at $1,500 \mathrm{xg}$ for $1 \mathrm{~min}$. The step was repeated with $0.5 \mathrm{ml} 70 \%$ acetonitrile/ $0.1 \%$ TFA and with $0.5 \mathrm{ml} 0.1 \%$ TFA. The peptide sample was loaded onto the cartridge followed by centrifugation at 150 to $500 \mathrm{xg}$ until the complete liquid was in the flow-through. The step was repeated with $0.5 \mathrm{ml}$ $0.1 \%$ TFA for washing. The cartridge was transferred into a new tube, and the previous centrifugation step was repeated with $0.5 \mathrm{ml} 70 \%$ acetonitrile for elution of the peptides. Samples were dried in the SpeedVac. A fraction of the sample was dried separately and reconstituted in LC-MS sample buffer ( $2 \%$ acetonitrile, $0.1 \%$ formic acid) for measurement of the peptide concentrations using the BCA assay as described before and with the MassPREP ${ }^{T M}$ E. coli Digest Standard (\#186003196, Waters, Eschborn, Germany) as reference.

\subsubsection{Phospho-peptide enrichment}

To enrich phospho-peptides from complex peptide mixtures, a protocol modified from Mazanek et al. (2007) was used. Peptide samples were reconstituted in $50 \mu 1$ loading solvent (70\% acetonitrile, $420 \mathrm{mM}$ 1-octanesulfonic acid (\#O0133, Sigma-Aldrich), $50 \mathrm{mg} / \mathrm{ml}$ dihydroxybenzoic acid (\#39319, Sigma-Aldrich), 0.1\% heptafluorobutyric acid (\#77249, Sigma-Aldrich), 3\% TFA) and applied onto equilibrated $\mathrm{TiO}_{2}$ columns (TT2TIO, Glygen Corporation, Columbia, Maryland, USA) in an estimated ratio of $400 \mu \mathrm{g}$ peptides per $1 \mathrm{mg}$ of $\mathrm{TiO}_{2}$ for optimized recovery of phospho-peptides from $\mathrm{TiO}_{2}$ according to Kanshin et al. (2013). Equilibration of the columns was done by applying $40 \mu \mathrm{l}$ of the first wash solution (70\% acetonitrile) followed by $40 \mu 1$ loading solvent. A syringe was used to gently press the liquid through the stationary phase. After loading of the peptide samples, columns were washed twice with $40 \mu \mathrm{l}$ loading solvent, once with the second washing solution (70\% acetonitrile, $125 \mathrm{mM}$ asparagine, $125 \mathrm{mM}$ glutamine, 3\% TFA), and twice with $40 \mu \mathrm{l}$ of the third washing solution (70\% acetonitrile, 3\% TFA). The second washing solution comprised asparagine and glutamine to displace N/Q-rich peptides on the $\mathrm{TiO}_{2}$ (Kanshin et al., 2013). Peptides were eluted from the column by applying two times $40 \mu 1$ elution buffer $(50 \mathrm{mM}$ ammonium dihydrogen phosphate adjusted to $\mathrm{pH} 10.5$ with ammonium hydroxide). Samples were acidified with $20 \mu 1$ TFA, dried, reconstituted in LC-MS sample buffer ( $2 \%$ acetonitrile, 
$0.1 \%$ formic acid), and again desalted using C18 cartridges. For LC-MS analyses, all samples were dissolved in $20 \mu 1$ sample buffer.

\subsubsection{Liquid chromatography-mass spectrometry analysis}

Liquid chromatography (LC) coupled to an Orbitrap Velos Pro ${ }^{\mathrm{TM}}$ Hybrid Ion Trap-Orbitrap mass spectrometer (MS) was employed for protein and phospho-peptide identification and for relative quantification by SILAC-ratios. Peptides of 1 to $6 \mu$ sample solution were trapped and washed on an Acclaim ${ }^{\circledR}$ PepMap 100 pre-column (\#164564, $100 \mu \mathrm{m}$ x 2 cm, C18, $3 \mu \mathrm{m}$, $100 \AA$, Thermo Fisher Scientific) at a flow rate of $25 \mu \mathrm{l} / \mathrm{min}$ for $6 \mathrm{~min}$ in $100 \%$ solvent A ( $2 \%$ acetonitrile, $0.07 \%$ TFA). Analytical peptide separation by reverse phase chromatography was performed on an Acclaim $^{\circledR}$ PepMap RSLC column (\#164540, $75 \mu \mathrm{m}$ x $25 \mathrm{~cm}$ or $50 \mathrm{~cm}, \mathrm{C} 18$, $3 \mu \mathrm{m}, 100 \AA$, Thermo Fisher Scientific) typically running a gradient from $98 \%$ solvent A ( $0.1 \%$ formic acid) and $2 \%$ solvent B (80\% acetonitrile, $0.1 \%$ formic acid) to $42 \%$ solvent B within $95 \mathrm{~min}$ and to $65 \%$ solvent B within the next $26 \mathrm{~min}$ at a flow rate of $300 \mathrm{nl} / \mathrm{min}$ (solvents and acids from Fisher Chemicals). Chromatographically eluting peptides were online ionized by nanoelectrospray (nESI) using the Nanospray Flex Ion Source (Thermo Fisher Scientific) at $2.4 \mathrm{kV}$ and continuously transferred into the mass spectrometer. Full scans within the mass range of 300 to $1850 \mathrm{~m} / \mathrm{z}$ were taken within the Orbitrap-FT analyzer at a resolution of 30,000 or 60,000 (SILAC experiments) with parallel data-dependent top ten MS2 collision-induced dissociation (CID) fragmentation with the LTQ Velos Pro linear ion trap. Phospho-peptide samples were analyzed with CID fragmentation applying the multistage activation (MSA) method as well as with higher energy collisional dissociation (HCD) fragmentation in a separate run. When HCD fragmentation was used, data-dependent top five MS2 fragmentation was performed, and fragment ions were analyzed in the Orbitrap. For analysis of Asc1p phospho-peptides, also targeted data acquisition with parent mass lists was applied. LC-MS method programming and data acquisition was done with the software XCalibur 2.2 (Thermo Fisher Scientific). For identification of Asc1p-derived phosphopeptides, MS/MS2 data were searched against an S. cerevisiae-specific protein databases (SGD, 6110 entries including common contaminants, S288C_ORF_database release version 2011, Stanford University) using the Proteome Discoverer Software version 1.4, and phospho-site localization was evaluated using phosphoRS (Olsen et al., 2006; Olsen and Mann, 2004). The digestion mode was trypsin, and a maximum of two missed cleavage sites was considered. Carbamidomethyl at cysteines was set as fixed modification. Oxidation at methionines and phosphorylation at serines, threonines, and tyrosines were considered as 
variable modifications. Mass tolerances of precursors and fragment ions were $10 \mathrm{ppm}$ and 0.6 Da, respectively. False discovery rates were calculated by the Proteome Discoverer using the reverse-decoy mode, and the filter for valid peptide sequence matches was set to 0.01 . For quantitative proteome analyses, MS/MS2 data were analyzed with the MaxQuant 1.5.1.0 software with the program's default parameters using an S. cerevisiae-specific protein database derived from UniProt (http://www.uniprot.org, Proteome ID UP000002311, 6721 entries, download 2014, Cox and Mann, 2008). The digestion mode was trypsin/P, and a maximum of two missed cleavage sites was considered. Carbamidomethyl at cysteines was set as fixed modification, and acetylation at the N-terminus, oxidation at methionines, phosphorylation at serines, threonines, and tyrosines (only for raw data from MSA and HCD LC-MS runs) were considered as variable modifications. Arg6 and Lys4 were defined as medium peptide labels and Arg10 and Lys8 as heavy peptide labels. Mass tolerances of precursors and fragment ions were $4.5 \mathrm{ppm}$ and $0.5 \mathrm{Da}$, respectively. Match between runs, requantification, and FTMS recalibration were enabled. For protein quantification, the minimum ratio count was two, and unique plus razor peptides were considered. False discovery rates were calculated by MaxQuant using the revert-decoy mode, and the filter for valid peptide sequence matches was set to 0.01. MaxQuant output data were further processed using the Perseus software 1.5.0.15 (Cox and Mann, 2008).

\subsection{RNA analyses}

\subsubsection{Northern blot analysis}

Total RNA was isolated from yeast according to Cross and Tinkelenberg (1991). $40 \mu \mathrm{g}$ RNA was mixed with $30 \mu 1$ sample buffer (66.7\% formamide, 9.25\% glycerol, $0.18 \%$ bromphenol blue, $1.8 \%$ ethidium bromide), heated at $65{ }^{\circ} \mathrm{C}$ for $15 \mathrm{~min}$, and kept on ice for $10 \mathrm{~min}$. RNA was separated on a $1.4 \%$ agarose gel containing $3 \%$ formaldehyde and $1 \% 3-(\mathrm{N}-$ morpholino)propanesulfonic acid (MOPS, $23 \mathrm{mM}$ MOPS, $5 \mathrm{mM}$ sodium acetate, 1 mM EDTA, pH 7) in running buffer (6.7 \% formaldehyde and 1\% MOPS) at $70 \mathrm{~V}$. Transfer of RNA onto a nylon membrane was performed by capillary blotting overnight. Cross-linking of the RNA to the membrane, generation and labeling of the probe, and signal detection were carried out as described for the Southern blot (chapter 2.7). 


\subsubsection{Sucrose density gradients}

Sucrose-density gradient fractionation experiments were performed as described by Mašek et al. (2011). S. cerevisiae cultures were grown overnight to midlog phase in $100 \mathrm{ml}$ liquid YNB medium. The culture was supplemented with cycloheximide at a final concentration of $100 \mu \mathrm{g} / \mathrm{ml}$ and incubated for $15 \mathrm{~min}$ on ice. Cells were harvested by centrifugation, resuspended in washing buffer (20 mM HEPES-KOH pH7.5, $10 \mathrm{mM} \mathrm{KCl,} 2.5 \mathrm{mM} \mathrm{MgCl}_{2}$, $1 \mathrm{mM}$ EGTA) and transferred into a $2 \mathrm{ml}$ reaction tube. After another round of centrifugation, the cells were resuspended in lysis buffer $(20 \mathrm{mM}$ HEPES-KOH pH 7.5, $10 \mathrm{mM} \mathrm{KCl}$, $2.5 \mathrm{mM} \mathrm{MgCl} 2,1 \mathrm{mM}$ EGTA, $1 \mathrm{mM}$ DTT, $100 \mu \mathrm{g} / \mathrm{ml}$ cycloheximide, $1.5 \mu \mathrm{l}$ RiboLock RNase Inhibitor (40 U/ $\mu 1$, \#EO0381, Thermo Fisher Scientific), 1 complete ${ }^{\mathrm{TM}}$ EDTA-free protease inhibitor tablet (\#05056489001, Roche Diagnostics) per $50 \mathrm{ml}, 1$ PhosSTOP ${ }^{\mathrm{TM}}$ phosphatase inhibitor cocktail tablet (\#04906837001, Roche Diagnostics) per $10 \mathrm{ml}$ ). The volume of lysis buffer and the amount of glass beads that were added equaled the volume of the cell pellet. Cells were disrupted using the Fast-Prep-24 (MP Biomedicals, Illkirch, France) two times for $20 \mathrm{sec}$ at $5.0 \mathrm{~m} / \mathrm{sec}$. Samples were centrifuged for $5 \mathrm{~min}$ at $13,000 \mathrm{rpm}$ at $4{ }^{\circ} \mathrm{C}$. The supernatant was transferred into a new reaction tube, and centrifugation was repeated for 7 min. The supernatant was again transferred into a new reaction tube, and the $\mathrm{OD}_{260}$ of a 1:100 dilution was measured with the NanoDrop 2000 (Thermo Scientific). A volume of cell extracts equaling an $\mathrm{OD}_{260}$ of 20 was loaded onto a 7 to $47 \%$ sucrose gradient. The gradient was generated with the Gradient Master 108 (BioComp Instruments, Fredericton, Canada) using $7 \%$ and $47 \%$ sucrose solutions prepared in the washing buffer. After centrifugation at $40,000 \mathrm{rpm}$ for $2 \mathrm{~h}$ and $50 \mathrm{~min}$ at $4{ }^{\circ} \mathrm{C}$ in a TH-641 rotor and a Sorvall WX80 ultracentrifuge, (Thermo Scientific) the gradient was fractionated using a fraction collector (Foxy Jr. Fraction Collector, Optical Unit Type 11, Absorbance detector UA-6, Teledyne Isco, Lincoln, Nebraska, USA) by pumping a $60 \%$ sucrose solution into the bottom of the tube. During fractionation, the absorbance at $254 \mathrm{~nm}$ was measured to monitor the distribution of RNA in the gradient and thus to obtain the polysome profile.

\subsubsection{Isolation of RNA from sucrose gradient fractions and mRNA enrichment}

Fractions from the sucrose-density gradient centrifugation were collected starting from the first polysome peak. 2 volumes of TRIzol ${ }^{\circledR}$ Reagent (\#15596-026, Thermo Fisher Scientific) were added to the samples followed by vigorous shaking. 0.2 volumes of $3 \mathrm{M}$ sodium acetate and 0.4 volumes of chloroform were added followed by 3 min shaking. Samples were then centrifuged at $4,200 \mathrm{rpm}$ for $30 \mathrm{~min}$ at $4{ }^{\circ} \mathrm{C}$. The upper aqueous phase was transferred into a 
new reaction tube, and $50 \mu \mathrm{g}$ glycogen (\#77534, Affymetrix, Cleveland, Ohio, USA) were added per $1 \mathrm{ml}$ of the sucrose gradient. Isopropanol was added in a 1:1 ratio. Samples were mixed and incubated overnight at $4{ }^{\circ} \mathrm{C}$. They were transferred into SS-34 tubes and centrifuged for $30 \mathrm{~min}$ at $13,000 \mathrm{rpm}$ in a Sorvall $^{\circledR} R C 5 B$ Plus centrifuge (Thermo Scientific). The supernatants were removed, and the pellets were washed with $25 \mathrm{ml}$ ice-cold $75 \%$ ethanol. The previous centrifugation step was repeated, and ethanol was removed. Pellets were dried and then resuspended in $250 \mu 1 \mathrm{H}_{2} \mathrm{O}$. The Oligotex Direct mRNA Kit (\#70022, Qiagen) was used for enrichment of mRNAs according to the provided Batch Procedure Protocol. Samples were eluted using two times $25 \mu$ Oligotex Elution Buffer. To remove DNA from the samples, digestion with DNase I (\#EN0521, Thermo Scientific) was performed. For digestion of $7.5 \mu \mathrm{l}$ sample from the previous elution step, $1 \mu \mathrm{l}$ DNase I, $1 \mu \mathrm{l}$ 10x reaction buffer with $\mathrm{MgCl}_{2}$ (\#B43, Thermo Scientific), and $0.5 \mu 1$ RiboLock RNase Inhibitor (40 U/ $\mu 1$, \#EO0381, Thermo Fisher Scientific) were added, and the samples were incubated at $37^{\circ} \mathrm{C}$ for $45 \mathrm{~min}$. Samples were then purified using the RNeasy MinElute Cleanup Kit (\#74204, Qiagen) according to the manufacturer's instructions.

\subsection{4 cDNA synthesis and RNASeq analysis}

The experimental steps and data analysis described in this chapter were performed together with the Department of Genomic and Applied Microbiology of the Institute of Microbiology and Genetics, Georg-August University Göttingen. Samples enriched for mRNA (see chapter 2.9.3) were subjected to cDNA synthesis using the NEBNext ${ }^{\circledR}$ Ultra $^{\mathrm{TM}}$ RNA Library Prep Kit for Illumina ${ }^{\circledR}$ (\#E7530S, New England Biolabs). After second strand cDNA synthesis, sample clean-up with the QIAquick PCR purification kit (\#28106; Qiagen) was performed. For the removal of adapter dimers and adapter monomers after library preparation, the Agencourt $^{\circledR}$ AMPure ${ }^{\circledR}$ XP Beads (\#A63881, Beckman Coulter, Krefeld, Germany) were applied. For the purification of cDNA, the GeneRead Size Selection Kit (\#180514, Qiagen) was used. All kits and reagents were used according to the manufacturer's instructions. Sequencing was performed on an Illumina Genome Analyzer IIx (Illumina, San Diego, USA). The sequence reads were mapped on the genome of S. cerevisiae (SGD) using Bowtie2 (Langmead and Salzberg, 2012) with standard parameters. The output Sequence Alignment/Map files were used to obtain readcount data for the $S$. cerevisiae genes to be used in a differential expression analysis applying the baySeq algorithm (Hardcastle and Kelly, 2010). Transcripts with a likelihood $\geq 90 \%$ and a false discovery rate $\leq 0.05$ were considered as differentially abundant in the analyzed translatomes. 


\subsection{Phenotypic tests}

Yeast cells were grown to midlog phase and diluted to an $\mathrm{OD}_{600}$ of 0.1 . Three consecutive ten-fold dilutions were prepared, and $10 \mu 1$ or $20 \mu 1$ of each dilution were dropped on YEP plus 2\% glucose/glycerol and on YNB medium, respectively. Media tested were YEP with $2 \%$ glycerol, YEPD with Congo red $(125 \mu \mathrm{g} / \mathrm{ml})$ or $\mathrm{NaCl}(75 \mathrm{mM})$, and YNB with canavanine $(600 \mathrm{ng} / \mathrm{ml})$ or cycloheximide $(0.15 \mu \mathrm{g} / \mathrm{ml})$. YEPD and YNB plates were used for growth controls. To assess the respiratory activity of the strains, a 2,3,5-triphenyltetrazolium chloride (TTC, \#93140, Sigma-Aldrich) assay (Ogur et al., 1957) was performed: $100 \mu \mathrm{l}$ of the highest dilution were streaked on YNB plates $(\varnothing 9.2 \mathrm{~cm})$ containing $0.4 \%$ glucose, and the plates were incubated for $3 \mathrm{~d}$ at $30^{\circ} \mathrm{C}$. Colonies were then overlaid with liquid $1.2 \%$ top agar containing $0.5 \%$ TTC and incubated for 20 to $30 \mathrm{~min}$ at $30{ }^{\circ} \mathrm{C}$. To observe glucosedependent colony morphology, $100 \mu 1$ of the highest dilution of the cell suspensions were streaked on YEP plates containing either $2 \%$ glucose or $0.4 \%$ glucose to obtain single colonies after $3 \mathrm{~d}$ growth at $30{ }^{\circ} \mathrm{C}$. To test the resistance of yeast strains against DETANONOate (\#82120, Cayman Chemical, Ann Arbor, Michigan, USA), an agar diffusion/halo assay was performed. Cells were grown to an $\mathrm{OD}_{600}$ of 0.6 in YNB medium, and $100 \mu 1$ of the culture were transferred to $10 \mathrm{ml} 0.5 \%$ liquid agar $\left(40{ }^{\circ} \mathrm{C}\right)$ that was poured onto $\mathrm{MV}$ plates after mixing. Two filter papers soaked with $2 \mu \mathrm{l}$ and $4 \mu \mathrm{l}$ of $1 \mathrm{M}$ DETA-NONOate, respectively, were immediately placed onto the agar surface. The ability of the yeast strains to grow adhesively was tested by growth on YNB plates containing $10 \mathrm{mM} \underline{3}$-amino-1,2,4triazole (3-AT, \#A8056, Sigma-Aldrich) at $30{ }^{\circ} \mathrm{C}$ for $3 \mathrm{~d}$ followed by washing of the plates under a constant stream of water. Plates were photographed using the Gel iX Imager. Colonies from the TTC and glucose-dependent colony morphology assays were documented using the stereomicroscope Olympus SZX12 (Olympus Corporation, Hamburg, Germany) and the cellSens Dimension software 1.4 (Olympus Corporation). 


\section{Results}

\subsection{Asc1p-dependent phenotypes}

Deletion of the RACK1 gene in metazoans leads to lethality during embryogenesis, whereas S. cerevisiae ASCl deletion strains are viable. Yeast $\Delta$ ascl strains exhibit multiple phenotypes related, for instance, to cell wall integrity, respiration, and translation. These strains are also deficient in the SNR24 gene that is located within the intron of the ASC1 gene and codes for the snoRNA U24 (Figure 9A, Chantrel et al., 1998). Thus, the absence of the snoRNA U24 in a $\Delta a s c 1$ strain might account for, or at least contribute to, the observed phenotypes.

The two major classes of snoRNAs are named according to conserved sequence motifs box C/D and H/ACA snoRNAs (Balakin et al., 1996; Ganot et al., 1997). The snoRNAs of the two families form ribonucleoprotein (RNP) complexes with distinct sets of proteins (reviewed in Watkins and Bohnsack, 2012). Members of the C/D snoRNP family catalyze 2'-O-ribosemethylation of pre-rRNA, whereas members of the H/ACA snoRNP family are responsible for pseudouridinylation of pre-rRNA (Kiss-László et al., 1996; Ni et al., 1997). Substrate specificity of the snoRNPs is provided by sequences in the snoRNA that are complementary to the modified rRNA target (Watkins and Bohnsack, 2012). The snoRNA U24 belongs to the C/D box family and is required for three site-specific 2'-O-ribose-methylations of $25 \mathrm{~S}$ prerRNA (Kiss-László et al., 1996). Although the SNR24 gene is highly conserved among eukaryotes, its position within an intron of $A S C 1 / R A C K 1$ is less well conserved. The human snoRNA U24 encoding gene, for instance, lies within the second intron of the RPL7A gene (Qu et al., 1995). The function of snoRNP U24 catalyzed 2'-O-methylation of 25S rRNA is not clear. The absence of snoRNA U24, but not the lack of Asc1p, leads to a halfmer phenotype in sucrose density gradients of S. cerevisiae cell extracts (Kouba et al., 2012). Halfmers are polysomes or monosomes bound to an additional 43S preinitiation complex, and they are detected as additional peaks in polysome profiles (Helser et al., 1981). The formation of halfmers might be due to reduced amounts of mature 60S ribosomal subunits in the cell as a consequence of loss of the snoRNA U24 (Rotenberg et al., 1988; Kouba et al., 2012).

To test whether also other phenotypes observed for the $\Delta a s c l$ strain are eventually caused by the deletion of the SNR24 gene, two different strains were generated: 1) a strain expressing the snoRNA U24, but not Asc1p, and 2) a strain only deleted for the SNR24-containing intron. For construction of the first strain, a $\operatorname{lox} P: \because U R A 3: \because \operatorname{lox} P$ cassette was inserted into exon 1 of the ASCl gene (Figure 9B). After the verification of transformants, the Cre-recombinase was 

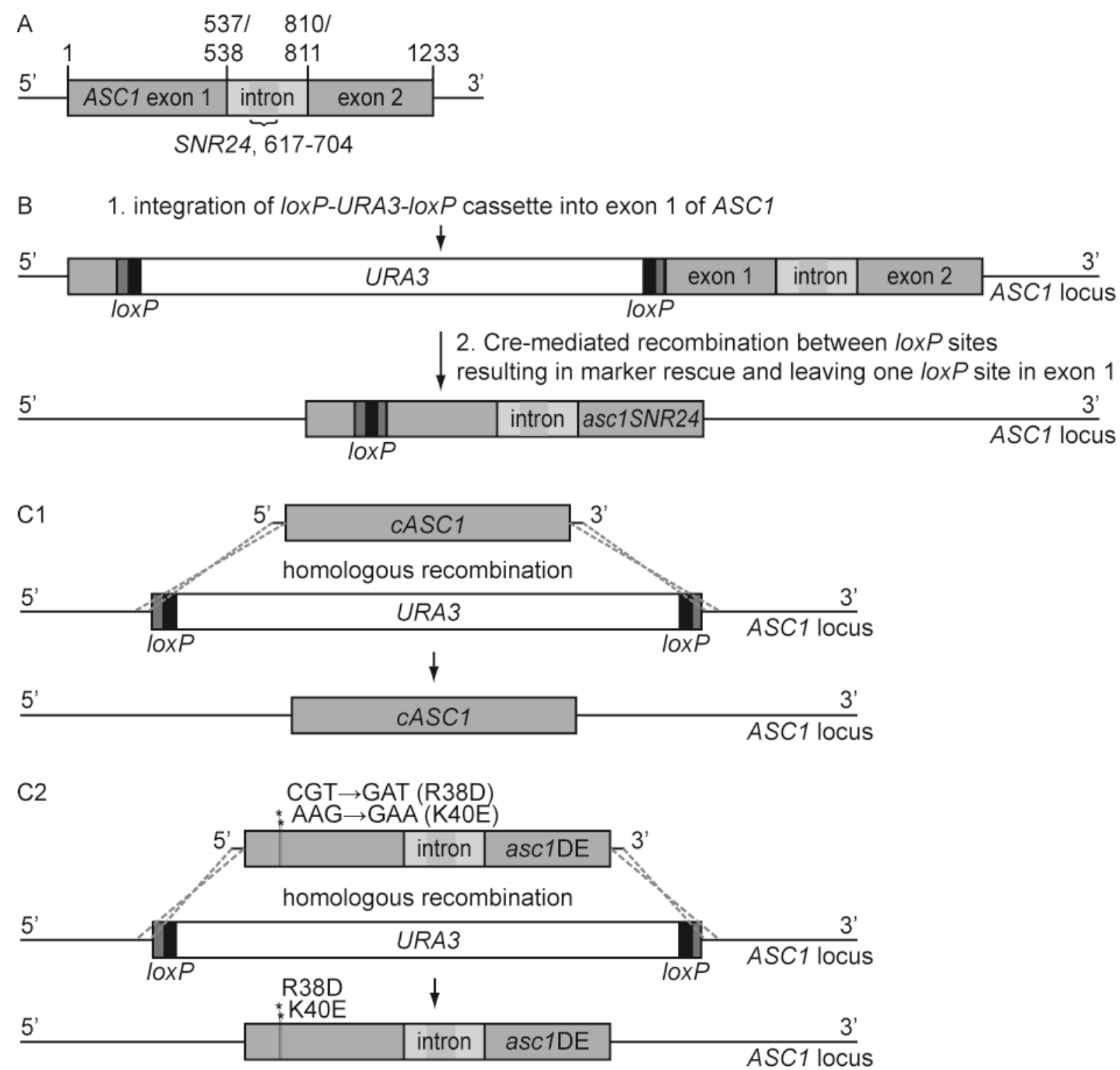

Figure 9. Construction of $S$. cerevisiae ASC1 and $S N R 24$ mutant strains.

(A) The genomic $A S C l$ locus. The $A S C l$ gene is interrupted by an intron that contains the snoRNA U24 encoding SNR24 gene. (B) Construction of a yeast strain still expressing the snoRNA U24, but no Asc1p. In the first step, the loxP-URA3-loxP cassette was introduced into exon 1 of the ASC1 gene between base pairs 164 and 165. After verification of the transformant, the marker was rescued through expression of plasmid-borne Cre-recombinase that mediates homologous recombination between the two loxP sites (indicated with black rectangles) flanking the URA3 gene. One loxP site of $34 \mathrm{bp}$ remains in exon 1 of the ascl ORF. The loxP site is flanked by two short regions (indicated with dark gray rectangles) that are derived from the amplification of the loxP-URA3-loxP cassette by PCR. In total, the sequence remaining in exon 1 has a size of $109 \mathrm{bp}$. The resulting strain is termed asclSNR24 or $\operatorname{ascl}^{-}$. (C) Introduction of mutated alleles of ASCl into the yeast genome at the native locus. For transformations, a $\triangle a s c 1:: U R A 3$ yeast strain was used, and the loxP-URA3-loxP cassette was replaced by different mutant alleles of the $A S C 1$ gene that were amplified from plasmids with $45 \mathrm{bp}$ overhangs homologous to the up- and down-stream regions of the $A S C 1$ gene. $\mathrm{C} 1$ shows the generation of a strain expressing the $A S C 1$ cDNA $(c A S C 1)$ without the intron, and $\mathrm{C} 2$ illustrates the construction of a yeast strain expressing mutated ascl with defined codon exchanges at specific positions within its ORF, such as R38D K40E. 
transiently expressed to induce homologous recombination between the two loxP sites flanking the URA3 marker, finally yielding in a single loxP site in exon 1 of $A S C 1$. The presence of this loxP site in the ascl mRNA should result in the abrogation of Asc1p synthesis during translation due to a stop codon within the loxP site (see Supplementary Figure 1). However, it should not interfere with the splicing of the mutated ASC1 pre-mRNA to generate functional snoRNA U24. The strain was designated asc1SNR24 and abbreviated as asc $^{-}$in the following chapters.

For the generation of the second strain, $A S C 1$ was amplified from a cDNA sample and cloned into a vector. The ASCI cDNA was then amplified from this plasmid with oligonucleotides that introduced 5' and 3' flanking regions for genomic integration through homologous recombination at the native $A S C 1$ locus. The replacement cassette was transformed into a $\triangle$ asc1::URA3 strain with ASC1 replaced by loxP::URA3::loxP (Figure 9C1). This enabled the selection of transformants on 5-fluoroorotic acid-containing media. The obtained verified strain was designated as $c A S C 1$. Both the $a s c 1^{-}$and the $c A S C 1$ strains were verified by PCR, Southern blot, and sequencing of the mutated locus. Furthermore, Northern blot experiments were performed to verify the expression of snoRNA U24 in the asc $^{-}$strain. Western blot experiments were performed to confirm the expression of Asc1p in the $c A S C 1$ strain and the absence of the protein in the $\mathrm{ssl}^{-}$strain (not all data shown, in part depicted in Figure 19). Both strains were analyzed for phenotypes that are known to rely on Asc1p and will be introduced in the following paragraphs. Total deletion of the ASC1 locus actually represents an ASC1 SNR24 double deletion, but is referred to as $\triangle a s c 1$ in this study.

Deletion of $A S C 1$ reduces the resistance of $S$. cerevisiae cells against translation inhibitors, such as cycloheximide, which interferes with the translocation step during elongation (Parsons et al., 2004). Also, the natural resistance against the non-proteinogenic amino acid canavanine is compromised in a $\Delta a s c l$ strain. The $\Delta a s c l$ strain is further impaired in its cell wall integrity that manifests in its increased sensitivity to Congo red, calcofluor white, or the glucanase zymolyase (Valerius et al., 2007; Rachfall et al., 2013). Deletion of ASC1 results in the loss of adhesive growth of haploid yeast cells and pseudohyphae formation of diploid cells due to reduced expression of the cell surface glycoprotein Flo11 (Valerius et al., 2007). The Aascl strain shows reduced growth on non-fermentable carbon sources, such as ethanol or glycerol, indicating impairment of respiration. This is further confirmed by strongly reduced red staining of $\Delta a s c 1$ cells through triphenyltetrazolium chloride (TTC), a compound that gets reduced by electrons of the respiratory chain leading to the formation of a red dye (Ogur et al., 1957). In the presence of high amounts of glucose (2\%), S. cerevisiae cells cover their energy 
supply mainly through fermentation. Therefore, the TTC assay is performed with colonies grown on medium containing low concentrations of glucose $(0.4 \%)$.

Impairment in respiration and low oxygen concentrations (hypoxia) can lead to the formation of reactive oxygen and nitrogen species (Figure 10A, Castello et al., 2006). These reactive compounds can lead to harmful modification of proteins and other cellular components. Asc1p-deficient cells show highly increased levels of the proteins Hbn1 and Yhb1, which are both assumed to function in the detoxification of such reactive compounds (Rachfall et al., 2013). Hbn1p (homologous to bacterial nitroreductases $\underline{1}$ ) is similar to bacterial nitroreductases, and cells deficient in this protein show decreased antioxidant activity, for example, mediated by the superoxide dismutase (Figure 10A, de Oliveira et al., 2010). Yhb1p (yeast flavohemoglobin $\underline{1}$ ) functions in the detoxification of nitric oxide (NO, Liu et al., 2000). NO inhibits the cytochrome c oxidase of the respiratory chain, and it reacts with superoxide to peroxynitrite, which causes protein tyrosine nitration (Brown, 2001; Radi, 2004). The increased sensitivity of the single deletion strains $\Delta h b n l, \Delta y h b l$, and $\Delta a s c l$ to the NO donor DETA-NONOate in a halo assay shows that all three proteins are required for resistance against nitrosative stress (Figure 10B). Double deletion strains of all three possible combinations of $\Delta a s c 1, \Delta h b n l$, and $\Delta y h b 1$ are more sensitive to nitrosative stress than the single deletion strains. A triple deletion mutant $\Delta a s c l \Delta h b n l \Delta y h b l$ was generated that is hypersensitive to DETA-NONOate. Strains with an ASCl deletion showed slightly impaired growth on the minimal medium used for the halo assay that might contribute to some minor extent to the reduced resistance against DETA-NONOate (Figure 10C). The data indicate that $\Delta$ ascl cells are prone to oxidative/nitrosative stress, such as externally applied NO. Loss of additional factors involved in the detoxification of reactive species, such as Hbn1p and Yhb1p, increases the sensitivity of $\triangle a s c 1$ cells to DETA-NONOate (Rachfall et al., 2013).

The ascl $^{-}$strain and the $c A S C l$ strain were analyzed with respect to a representative selection of the described phenotypes (Figures 10 and 11). The ascl ${ }^{-}$strain behaved identical to the $\triangle a s c 1$ strain and the $c A S C 1$ strain identical to the wild-type ASCl strain. Thus, the phenotypes of a $\Delta a s c 1$ strain observed here are caused by the absence of the Asc1 protein and not by the lack of the snoRNA U24. To exclude any impact of a loss of snoRNA U24 on observations made for Asc1p-deficient strains, the $a s c 1^{-}$strain was used instead of the $\Delta a s c 1$ strain for subsequent experiments in this study. 


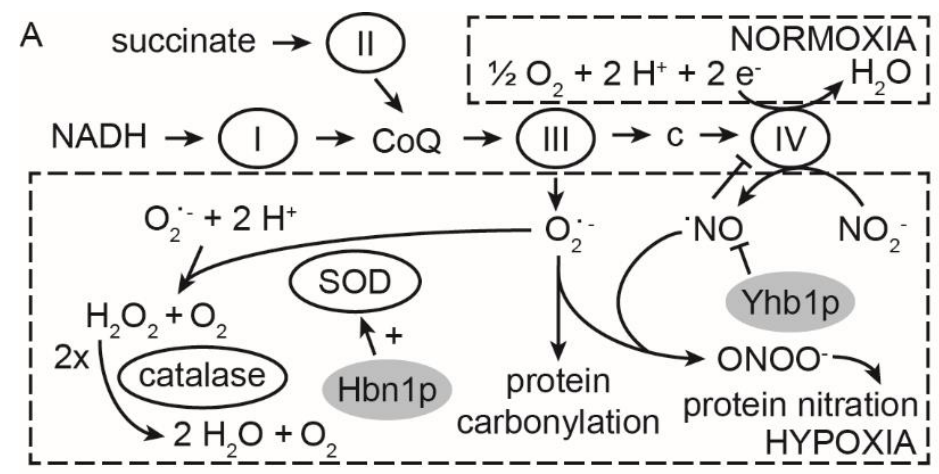

C

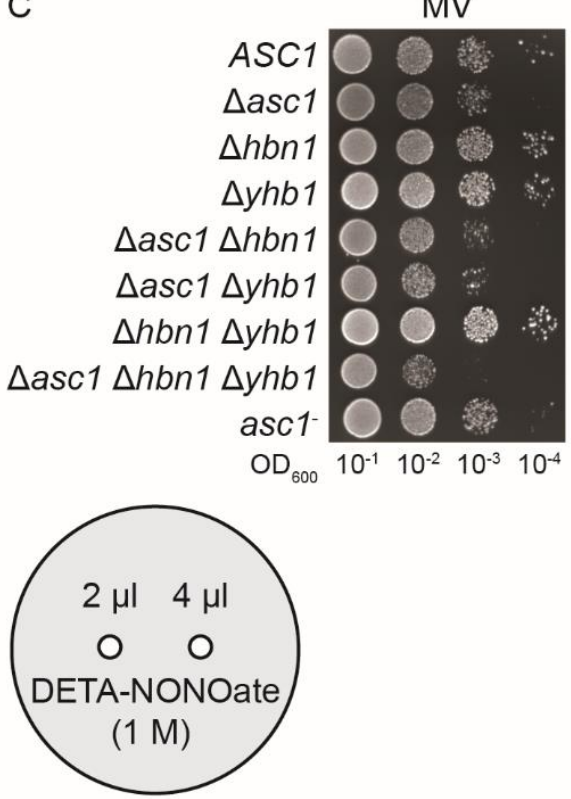

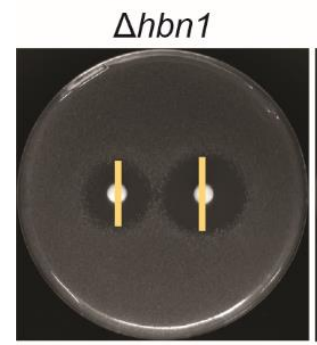

$\Delta a s c 1 \Delta h b n 1$

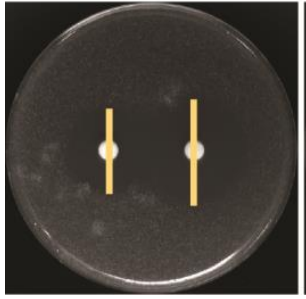

$\Delta$ asc1

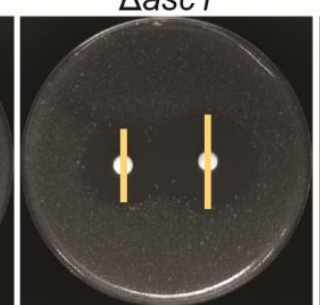

$\Delta y h b 1$

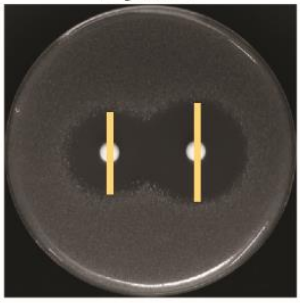

$\Delta a s c 1 \Delta y h b 1$

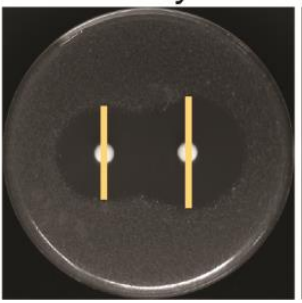

$\operatorname{asc1}^{-}$

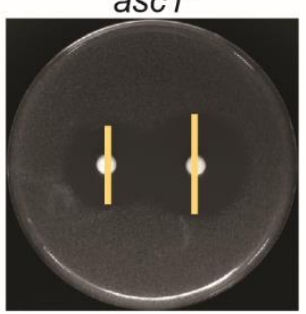

$\Delta h b n 1 \Delta y h b 1$

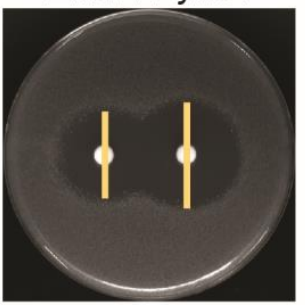

$\Delta a s c 1 \Delta h b n 1 \Delta y h b 1$

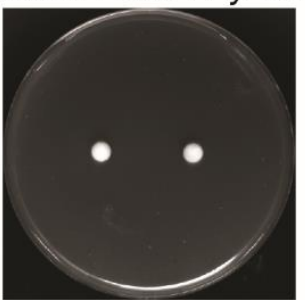

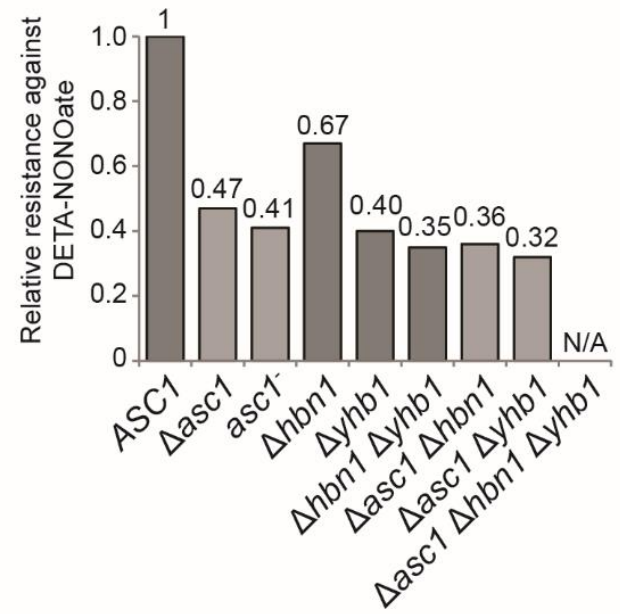

Figure 10. Nitrosative stress in Asc1p-deficient strains.

(A) The generation of reactive oxygen and nitrogen species within the respiratory chain upon hypoxia. Under normoxia, the cytochrome c oxidase complex (IV) of the respiratory chain transfers electrons onto oxygen leading to the formation of water molecules. Upon hypoxia, electrons are also transferred to nitrite $\left(\mathrm{NO}_{2}{ }^{-}\right)$yielding in nitric oxide ( $\left.\mathrm{NO}\right)$. Furthermore, accumulating electrons on the cytochrome bc1 complex (III) are released by superoxide $\left(\mathrm{O}_{2}{ }^{-*}\right)$ formation. $\mathrm{NO}$ and $\mathrm{O}_{2}{ }^{--}$react to form peroxynitrite $\left(\mathrm{ONOO}^{-}\right)$, which can modify proteins through tyrosine nitration. $\mathrm{O}_{2}^{--}$can be detoxified through the enzymes superoxide dismutase (SOD) and catalase. Yhb1p can consume $\mathrm{NO}$, and Hbn1p seems to be involved in activation of SOD (Liu et al., 2000; de Oliveira et al., 2010). (NADH = nicotinamide adenine dinucleotide, $\mathrm{CoQ}=$ coenzyme $\underline{\mathrm{Q}}, \mathrm{c}=$ cytochrome $\underline{\mathrm{c}} ; \mathrm{I}=\mathrm{NADH}$ coenzyme $\mathrm{Q}$ reductase, $\mathrm{II}=$ succinate dehydrogenase; modified from Castello et al., 2006). (B) Agar diffusion/halo assay to determine the sensitivity to the nitric oxide donor DETA-NONOate. Yeast cells were diluted in liquid top agar that was poured on MV plates ( $\mathrm{pH} 7.2$ ), and filter papers containing $2 \mu 1$ or $4 \mu \mathrm{l}$ of $1 \mathrm{M}$ DETA-NONOate were applied. Plates were incubated for 2 days at $30^{\circ} \mathrm{C}$, and the size of growth inhibition zones was determined. The graph shows the resistance of the deletion strains against DETANONOate relative to the wild-type determined by the reciprocal values of the halo diameters. (C) Drop dilution assay. Serial ten-fold dilutions of the cell suspensions used for the halo assay were dropped onto MV-plates to evaluate total growth of the strains on this medium. (N/A = not applicable; modified from Rachfall et al., 2013). 

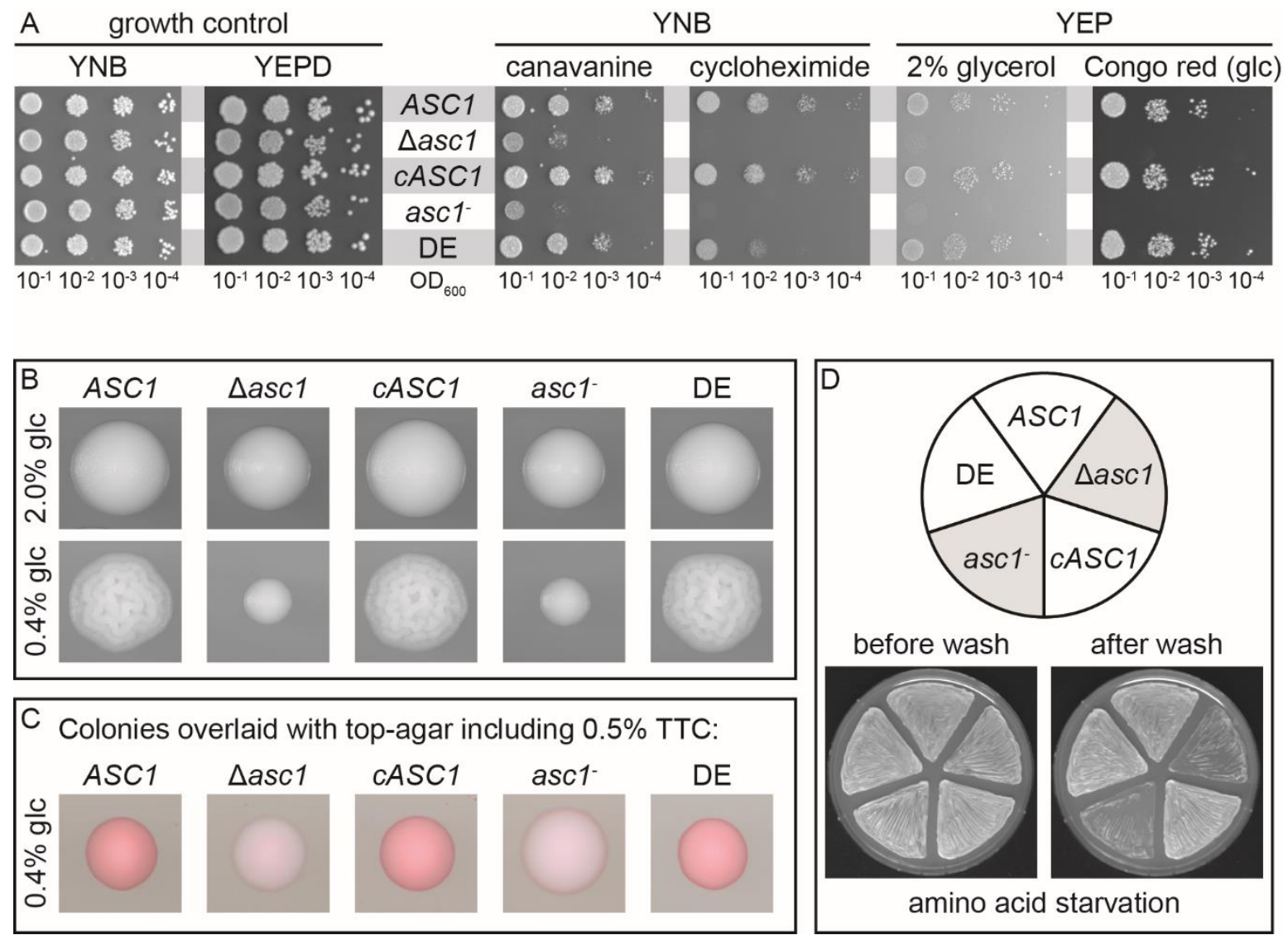

in acid starvation

Figure 11. Phenotypic characterization of the asc1SNR24, cASC1, and asc1DE strains.

(A) Drop-dilution assays. Cell suspensions were spotted onto different growth media. YNB media containing $600 \mathrm{ng} / \mathrm{ml}$ canavanine or $0.15 \mu \mathrm{g} / \mathrm{ml}$ cycloheximide were used to test strains for their sensitivity to inhibitors of protein biosynthesis. To evaluate the growth of strains on non-fermentable carbon sources, cells were grown on YEP medium containing 2\% glycerol as sole carbon-source. Sensitivity to cell wall stress was analyzed through growth on $125 \mu \mathrm{g} / \mathrm{ml}$ Congo red containing YEPD plates $(\mathrm{glc}=$ glucose $)$. YNB and YEPD plates served as growth controls. (B) Colony morphology under low glucose. Cells were spread on YEP plates containing $2 \%$ glucose (high glucose) or $0.4 \%$ glucose (low glucose) to form single colonies with distinct morphologies after $3 \mathrm{~d}$ growth at $30^{\circ} \mathrm{C}$. (C) TTC assay to evaluate respiratory activity. Cells were spread on YNB plates containing low amounts of glucose $(0.4 \%)$. After $3 \mathrm{~d}$ incubation at $30{ }^{\circ} \mathrm{C}$, colonies were overlaid with top-agar containing $0.5 \%$ TTC that is reduced by electrons of the active respiratory chain to a red dye. (D) Test for adhesive growth. Haploid cells were patched onto $10 \mathrm{mM} 3$-AT containing YNB plates and incubated for $3 \mathrm{~d}$ at $30^{\circ} \mathrm{C}$. Afterwards, plates were washed under a constant stream of water to remove non-adherent cells from the agar surface. Non-adherent strains are highlighted in gray.

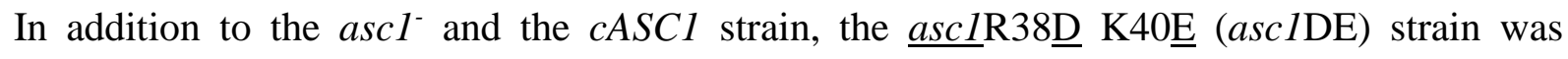
included in the phenotypic characterizations. This strain expresses an Asc1p variant that is supposed to bind less efficiently to the ribosome due to the exchange of two highly conserved, positively charged residues R38 and K40 to negatively charged aspartate (D) and glutamate (E, Coyle et al., 2009). R38 of Asc1p seems to contact D27 of Rps17p, and K40 appears to form a salt-bridge with a phosphate residue of the sugar-phosphate backbone of the rRNA (Adams et al., 2011). An S. cerevisiae asclDE strain has originally been constructed and 


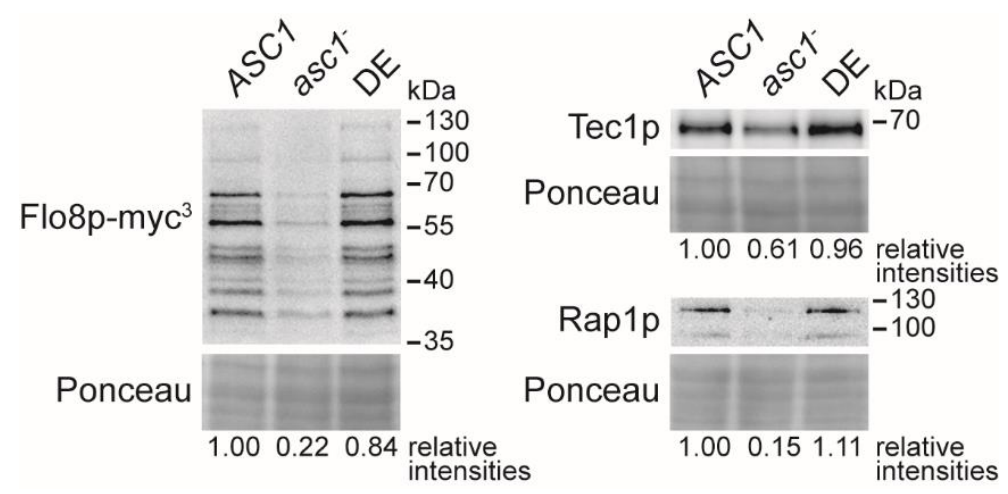

Figure 12. Asc1p-dependent transcription factor levels.

Immunodetection of Flo8p-myc ${ }^{3}$, Tec1p, and Rap1p within cell extracts from ASCl wild-type, asc1and asc IDE cultures. A c-myc antibody was used to detect myc ${ }^{3}$-tagged Flo8p. Tec1p and Rap1p were detected using protein-specific antibodies. Ponceau red staining of proteins served as loading control, and a part of the lower half of the stained Western blot membrane is depicted. Fold changes relative to wild-type signal intensities are given below each lane.

phenotypically characterized by Coyle and colleagues (2009). They observed a shift of the majority of Asc1DEp from the ribosomal to the ribosome-free fraction during sucrose gradient ultracentrifugation. Nevertheless, the asclDE strain was shown to behave phenotypically mainly like the $A S C l$ wild-type strain, apart from the staining of cells with calcofluor white that revealed elevated levels of chitin in the cell wall similar to an ASC1 deletion strain (Coyle et al., 2009). Here, an asclDE mutant strain was generated as well in the used S. cerevisiae $\Sigma$-strain background (Figure 9C2) and tested for additional phenotypes. Similar to what was observed by Coyle and colleagues, the asclDE strain behaved mostly like the ASC1 wild-type strain. However, it showed a slightly reduced resistance against cycloheximide (Figure 11). Loss of Asc1p causes decreased abundance of the transcription factors Flo8p, Tec1p, and Rap1p. The asc $1^{-}$strain confirms that this effect depends on the lack of Asc1p and not on the absence of the snoRNA U24. The abundance of these transcription factors, however, remained unaffected in the asc1DE strain (Figure 12). Altogether, these observations indicate that compromised binding of Asc1p to the ribosome due to the DE exchange only slightly reduces the functionality of the protein.

\subsection{Phosphorylation of Asc1p}

Asc1p/RACK1 is known to interact with protein kinases both in yeast and higher eukaryotes. The phosphorylation of Asc1p itself likely regulates its protein-interactions, stability, and subcellular localization (Chang et al., 2002; Zeller et al., 2007). Phospho-sites within Asc1p have been identified in high throughput studies (Smolka et al., 2007; Holt et al., 2009). Here, phosphorylation of Asc1p was studied with a targeted approach based on the purification of 
the protein from yeast cultures and identification of phospho-peptides using LC-MS. Subsequently, S. cerevisiae mutant strains were generated with amino acid exchanges at the newly identified and the previously known phospho-sites of Asc1p and subjected to phenotypic characterizations to reveal the impact of phosphorylation on the functionality of Asc1p.

\subsubsection{The surface of Asc1p is target for multiple phosphorylations}

Strep-tagged Asc1p was purified from exponentially growing S. cerevisiae cell cultures, and tryptic peptides of the protein were analyzed with LC-MS for in-depth characterization of its phosphorylation. The raw data search against an S. cerevisiae-specific protein database using the Proteome Discoverer 1.4 software and the SequestHT and Mascot search algorithms resulted in the identification of seven singly phosphorylated peptides, two of them with an overlapping sequence due to a trypsin missed cleavage site at K161 (Figure 13). Since all phospho-peptides identified contained more than one serine, threonine, and/or tyrosine residue, the phosphoRS algorithm was used to calculate phospho-site probabilities (Olsen and

\begin{tabular}{|c|c|c|c|c|c|}
\hline & & & WD1 & & \\
\hline $7 \beta D$ & $1 \beta A$ & $1 \beta B$ & $1 \beta C$ & $1 \beta D$ & $2 \beta A$ \\
\hline
\end{tabular}

MASNEVLVLRGTLEGHNGWVTSLATSAGQPNLLLSASṚDḲTLISWKLTGDDQKFGVPVRSFKGHSHIVQDCTLTADGAYĀ

\begin{tabular}{|c|c|c|c|c|c|c|c|c|}
\hline \multicolumn{2}{|r|}{ WD2 } & \multicolumn{7}{|c|}{ WD3 } \\
\hline $2 \beta B$ & $2 \beta C$ & $2 \beta D$ & $3 \beta A$ & $3 \beta B$ & $3 \beta C$ & $3 \beta D$ & $4 \beta A$ & \\
\hline & & & & & & 143 & * & 160 \\
\hline
\end{tabular}

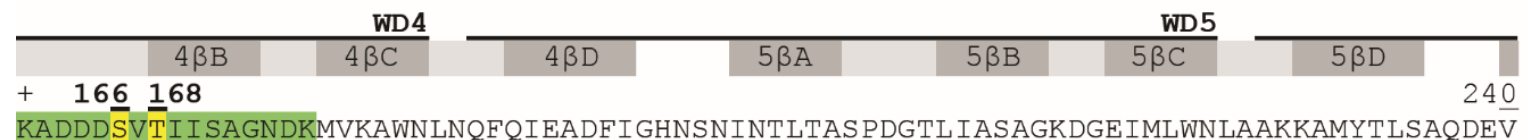

KADDDSVTI ISAGNDKMVKAWNLNOFOIEADFI GHNSNINTLTAS PDGTLIASAGKDGEIMLWNLAAKKAMYTLSAODEV

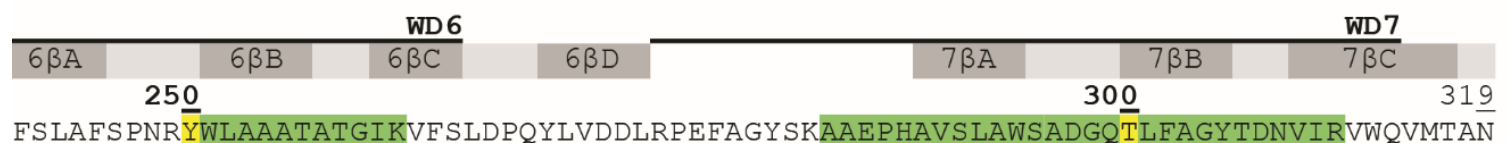

$$
\begin{aligned}
& =\text { identified phospho-peptide } \quad=\beta \text {-propeller blade } \quad \ldots \ldots=\text { uncovered amino acid sequence } \\
& =\text { phospho-site } \quad=\beta \text {-sheet } \quad+=\text { missed cleavage site } \\
\text { * } & =\text { cleavage site between two adjacent phospho-peptides }
\end{aligned}
$$

Figure 13. LC-MS-based identification of Asc1p phospho-sites.

Asc1p amino acid sequence is depicted with identified phosphorylated peptides and sites. The amino acid sequence coverage of Asc1p considering all identified high confident peptides was $97.81 \%$ (false discovery rate $\leq$ 0.01). Phospho-sites S166 and T168 were identified within a phospho-peptide ranging from residue A162 to K176 and within a second peptide from residue V156 to K176 bearing a missed cleavage site at the C-terminus of K161 that is indicated with a plus (+). Residues R38 and K40, which lead to compromised ribosome-association of Asc1p when mutated to D and E, respectively, are written in red and marked with dots (Sengupta et al., 2004; Coyle et al., 2009). WD40 repeats are indicated according to SGD (SMART domain SM0032). See Supplementary Table 1 for details on the identification of phospho-peptides and Supplementary Figure 2 for fragmentation spectra. 
Mann, 2004; Olsen et al., 2006). Six amino acid residues of Asc1p, T12, S120, T143, S166, T168, and Y250, were identified with a site probability of $100 \%$ to be unambiguous phosphosites. These data confirmed the already known phospho-sites S120, S166, and T168 (Smolka et al., 2007; Holt et al., 2009) and led to the identification of three previously unknown sites, T12, T143, and Y250. Furthermore, a phospho-peptide was identified with $99.9 \%$ as the highest localization probability for residue T300. For more details on peptide identification and phospho-site localization, see Supplementary Table 1 and Supplementary Figure 2. This study focused on T12, S120, T143, S166, T168, and Y250 as well as on T96 and T99 (Chi et al., 2007) that were not detected in the experiments presented here. The position of these eight phospho-sites within the protein bound to the $40 \mathrm{~S}$ subunit of the ribosome is illustrated in Figure 14. T12, T99, and T143 are localized in the $\beta$ D-strands of blades 7, 2, and 3 thus being localized at the circumference of the protein. T96, S120, S166, and Y250 are localized at sides of Asc1p that do not directly face the ribosome. T96 lies next to T99 in close proximity to the ribosomal protein Rps16. S166 is positioned within the loop between $\beta \mathrm{A}-$ and $\beta \mathrm{B}-$ strands of blade 4 adjacent to T168, which is the N-terminal amino acid of strand $4 \beta \mathrm{B}$. None of the phospho-sites is localized at the side of Asc1p known to directly contact other ribosomal proteins indicating that kinases can access the protein in its ribosome-bound state.

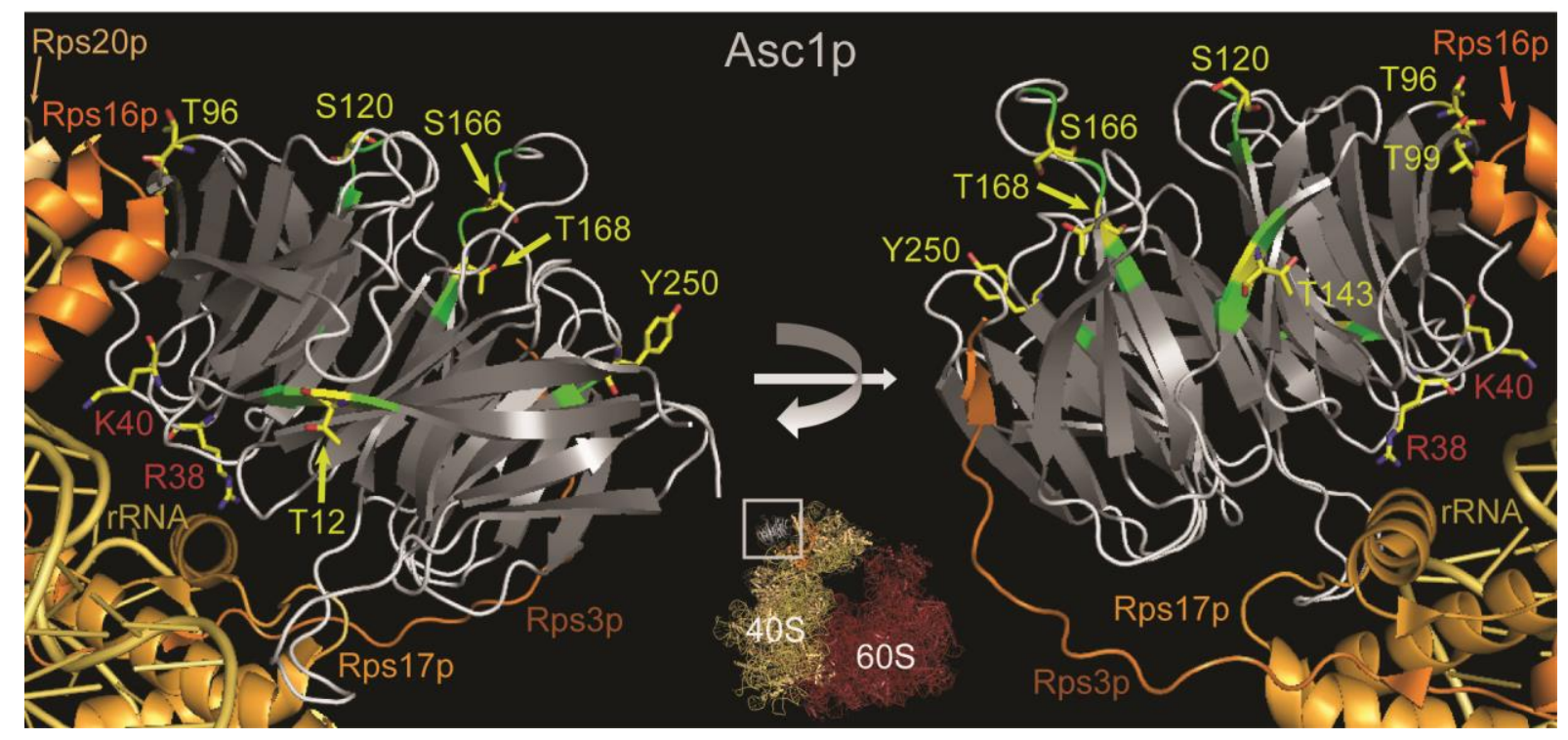

Figure 14. Cartoon view of Asc1p bound to the 40S subunit of the ribosome.

The Asc1p $\beta$-propeller is depicted in white (loops) and gray ( $\beta$-strands). Positions of phospho-sites that were further analyzed in this study are highlighted in yellow, and the amino acids are depicted as sticks with carbon atoms in yellow, nitrogen atoms in blue, and oxygen atoms in red. For better recognizability, neighboring amino acids within the LC-MS-identified peptides are colored in green. Additionally, residues R38 and K40 are highlighted. For the ribosomal protein Rps3, the C-terminal last 14 amino acids were structurally not resolved and are therefore missing. Figures of structures were generated with PyMOL Molecular Graphics System software on the basis of the PDB file 4V88 (BenShem et al., 2011). 


\subsubsection{Construction of yeast strains expressing Asc1p isoforms with amino acid exchanges}

\section{at phospho-sites}

To analyze the impact of phosphorylation on Asc1p, S. cerevisiae Asc1p phospho-site mutant strains were generated. These strains express Asc1p having either glutamate or alanine at one of the phospho-sites to mimic their constitutively phosphorylated or dephosphorylated state, respectively. Y250 was mutated to phenylalanine instead of alanine due to the higher similarity between these two amino acids. For phospho-sites lying close to each other within the amino acid sequence (T96 and T99 as well as S166 and T168), strains with both sites mutated simultaneously were generated in addition.

Furthermore, strains carrying each phospho-site substitution in combination with the two amino acid exchanges R38D K40E were constructed. These two amino acid exchanges are considered to compromise the ribosome binding of Asc1p (see chapter 3.1). The DE mutation causes only a marginal loss of Asc1p's functionality, but it might cause synthetic effects in combination with the phospho-site mutations. All constructed strains are listed in Table 1 . Growth phenotypes studied with these strains are summarized in Table 3 and are illustrated and described in the following chapter.

\subsubsection{Asc1p phospho-sites T143 and Y250 confer resistance against translation inhibitors and together with T12, T96, and T99 are essential to maintain overall functionality of the Asc1DEp variant}

The phospho-site mutant strains constructed were analyzed with respect to the Asc1pdependent phenotypes introduced in chapter 3.1 and the $A S C 1$ wild-type, asc $1^{-}$, and asclDE strains were used as references. Figures 15-17 give an overview of the main observations. The dephosphorylation mimicking mutations $\mathrm{T} 143 \mathrm{~A}$ and $\mathrm{Y} 250 \mathrm{~F}$ increased the sensitivity to cycloheximide similar to the DE mutation, and the Y250F mutation also caused sensitivity to canavanine (Figure 15). In the absence of an additional DE mutation, all other phospho-site mutant strains showed wild-type behavior (Figures 15-17 and data not shown). DE-compromised localization of Asc1p at the ribosome, however, enhances the effects caused by amino acid exchanges at T143 and Y250. The $a s c 1^{\mathrm{T} 143 \mathrm{~A}} \mathrm{DE}$ and $a s c 1^{\mathrm{Y} 250 \mathrm{~F}} \mathrm{DE}$ strains showed synthetic hypersensitivity to cycloheximide in comparison to the strains bearing only the dephosphorylation mimic or the DE mutation (Figure 15). They were even phenotypically similar to the ascl $^{-}$strain. Irrespective of the DE mutation, phenylalanine at position 250 affected the sensitivity to cycloheximide and canavanine. The ascl ${ }^{\mathrm{T} 143 \mathrm{~A}} \mathrm{DE}$ strain behaved similar to the asc $^{-}$strain in all tests showing, for example, strongly reduced red pigmentation 
Table 3. Overview of Asc1p phospho-site mutant strains and their phenotypes.

As references the phenotypes of the $A S C 1$ wild-type strain were defined as + , and the phenotypes of the Asc1p-deficient ascl- strain as - - - The phenotypes of the Asc1p phospho-site mutant strains were classified accordingly. Dark gray background color highlights strains and their phenotypes that differed significantly from the $A S C 1$ wild-type strain.

\begin{tabular}{|c|c|c|c|c|c|c|c|}
\hline & $\begin{array}{l}\text { cana- } \\
\text { vanine }\end{array}$ & $\begin{array}{c}\text { cyclo- } \\
\text { heximide }\end{array}$ & glycerol & $\begin{array}{l}\text { TTC } \\
\text { assay }\end{array}$ & $\begin{array}{l}\text { Congo } \\
\text { red }\end{array}$ & $\begin{array}{l}\text { wrinkled } \\
\text { colonies }\end{array}$ & $\begin{array}{l}\text { adhesive } \\
\text { growth }\end{array}$ \\
\hline \multicolumn{8}{|l|}{ reference strains } \\
\hline$A S C 1$ & + & + & + & + & + & + & + \\
\hline asc $^{-}$ & $-\cdots$ & $-\cdots$ & --- & $-\cdots$ & $-\ldots$ & $-\cdots$ & $-\ldots$ \\
\hline asc1DE & + & - & + & + & + & + & + \\
\hline \multicolumn{8}{|l|}{$\begin{array}{l}\text { phospho-site } \\
\text { mutant strains }\end{array}$} \\
\hline T12A & + & + & + & + & + & + & + \\
\hline T12A DE & + & - & + & + & + & + & - \\
\hline $\mathrm{T} 12 \mathrm{E}$ & + & + & + & + & + & + & + \\
\hline T12E DE & + & - & + & + & + & + & + \\
\hline T96A & + & + & + & + & + & + & + \\
\hline T96A DE & - & - - & - - & - & - & - - & -- \\
\hline T96E & + & + & + & + & + & + & + \\
\hline T96E DE & - & - - & - - & - & - & - & - - - \\
\hline T99A & + & + & + & + & + & + & + \\
\hline T99A DE & + & - & + & + & + & - & - \\
\hline T99E & + & + & + & + & + & + & + \\
\hline T99E DE & + & - & + & + & + & + & + \\
\hline T96A T99A & + & + & + & + & + & + & + \\
\hline T96A T99A DE & - - & $--(-)$ & -- & - - & -- & - - & -- \\
\hline T96E T99E & + & + & + & + & + & + & + \\
\hline T96E T99E DE & - & + & + & + & - & - & $-\ldots$ \\
\hline S120A & + & + & + & + & + & + & + \\
\hline S120A DE & + & - & + & + & + & + & + \\
\hline S120E & + & + & + & + & + & + & + \\
\hline T143A & + & - & + & + & + & + & + \\
\hline T143A DE & $--(-)$ & - & -- &.- & -- & -- & $-\cdots$ \\
\hline T143E & + & + & + & + & + & + & + \\
\hline T143E DE & - & $-\cdots$ & + & -- & + & - & -- \\
\hline S166A & + & + & + & + & + & + & + \\
\hline S166A DE & + & - & + & + & + & + & + \\
\hline S166E & + & + & + & + & + & + & + \\
\hline T168A & + & + & + & + & + & + & + \\
\hline T168A DE & + & - & + & + & + & + & + \\
\hline T168E & + & + & + & + & + & + & + \\
\hline S166A T168A & + & + & + & + & + & + & + \\
\hline T166A T168A DE & + & - & + & + & + & + & + \\
\hline S166E T168E & + & + & + & + & + & + & + \\
\hline Y250F & - & - & + & + & + & + & + \\
\hline Y250F DE & -- & -- & + & + & + & + & + \\
\hline
\end{tabular}




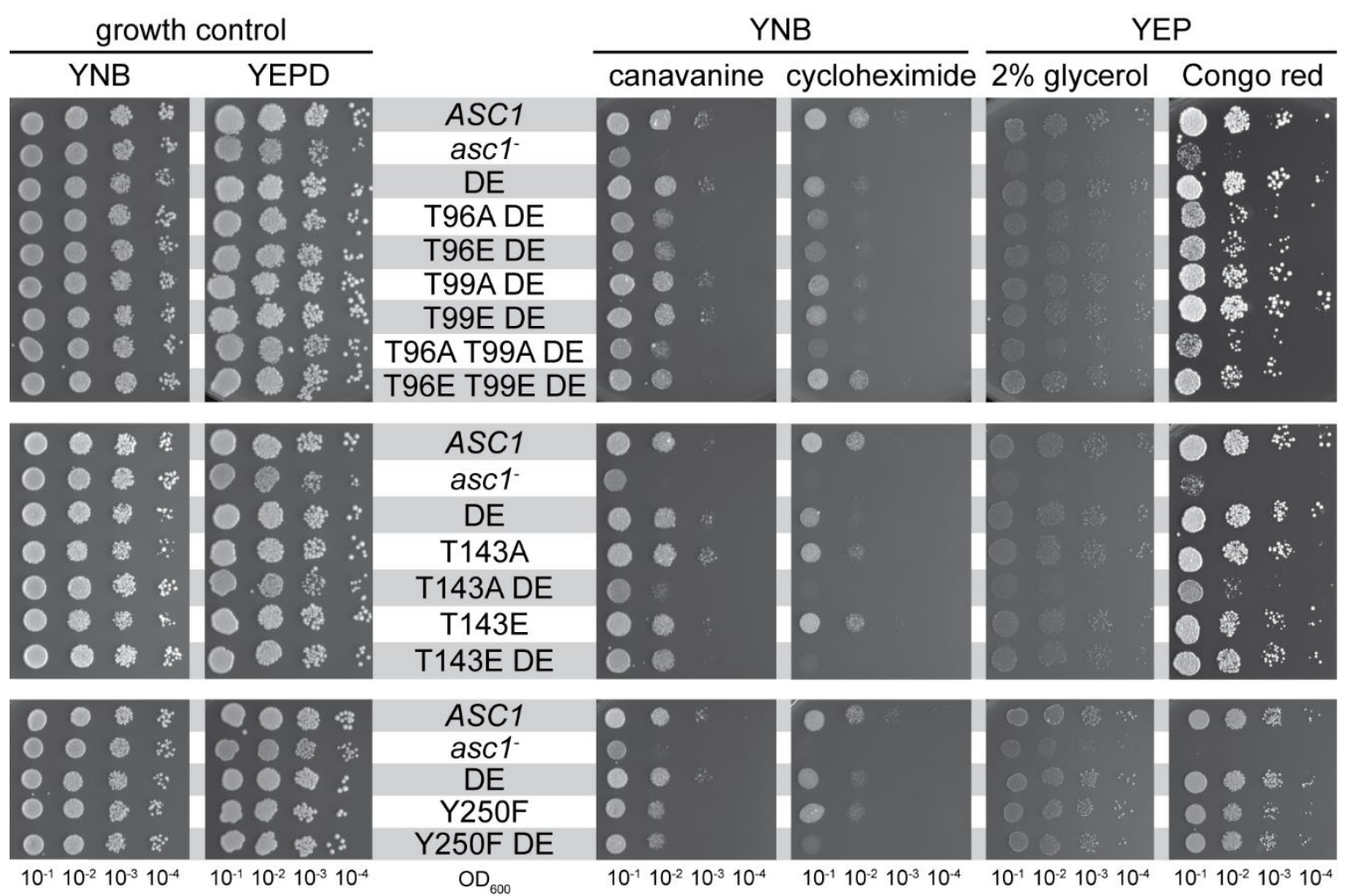

Figure 15. Drop dilution assays to analyze the impact of phospho-site mutations on Asc1pdependent phenotypes.

Cell suspensions were spotted on YNB plates with or without $0.15 \mu \mathrm{g} / \mathrm{ml}$ cycloheximide or $600 \mathrm{ng} / \mathrm{ml}$ canavanine, respectively, to assess sensitivity to translation/protein biosynthesis inhibitors. To evaluate cell wall integrity, cells were dropped on YEPD plates with or without $125 \mu \mathrm{g} / \mathrm{ml}$ Congo red, and to test fermentative activity, cells were spotted onto YEP medium with $2 \%$ glycerol instead of glucose.

in the presence of TTC (Figure 16B2). Only its sensitivity to Congo red was less pronounced than for the $a s c 1^{-}$strain. The $a s c 1^{\mathrm{T} 143 \mathrm{E}} \mathrm{DE}$ strain with a phosphorylation mimic at position 143 showed similar but in most cases milder phenotypes than the $\operatorname{ssc}{ }^{\mathrm{T} 143 \mathrm{~A}} \mathrm{DE}$ strain.

Combination with the DE mutation also caused strong effects of the amino acid substitutions at T12, T96, and T99. The $a s c 1^{\mathrm{T} 12 \mathrm{~A}} \mathrm{DE}$ strain showed impaired adhesive growth (Figure 17). The $a s c 1^{\mathrm{T} 96 \mathrm{~A}} \mathrm{DE}$ and the $a s c 1^{\mathrm{T} 96 \mathrm{E}} \mathrm{DE}$ strain were non-adhesive and showed no wrinkled colony morphology on $0.4 \%$. Other phenotypes were only mildly affected (Figures 15-17). The substitution of T99 by alanine, but not by glutamate, combined with the DE mutation affected the same two phenotypes though less severely. The $a s c 1^{\mathrm{T} 96 \mathrm{~A}}{ }^{\mathrm{T} 99 \mathrm{~A}} \mathrm{DE}$ strain with both neighboring amino acids replaced by alanine behaved like the $\operatorname{asc} 1^{\mathrm{T} 96 \mathrm{~A}} \mathrm{DE}$ strain except for its slightly increased sensitivity to cycloheximide (Figure 15). Interestingly, the sensitivity of the asc $1^{\mathrm{T} 96 \mathrm{E}} \mathrm{DE}$ strain to cycloheximide and its reduced growth on the non-fermentable carbon source glycerol were suppressed by an additional T99E exchange (ascl ${ }^{\mathrm{T} 96 \mathrm{E}}{ }^{\mathrm{T} 99 \mathrm{E}} \mathrm{DE}$ strain). In all other assays, the additional T99E mutation only partially suppressed the phenotype caused 
A Wrinkled colony morphology on YEP plates containing $0.4 \%$ glc:
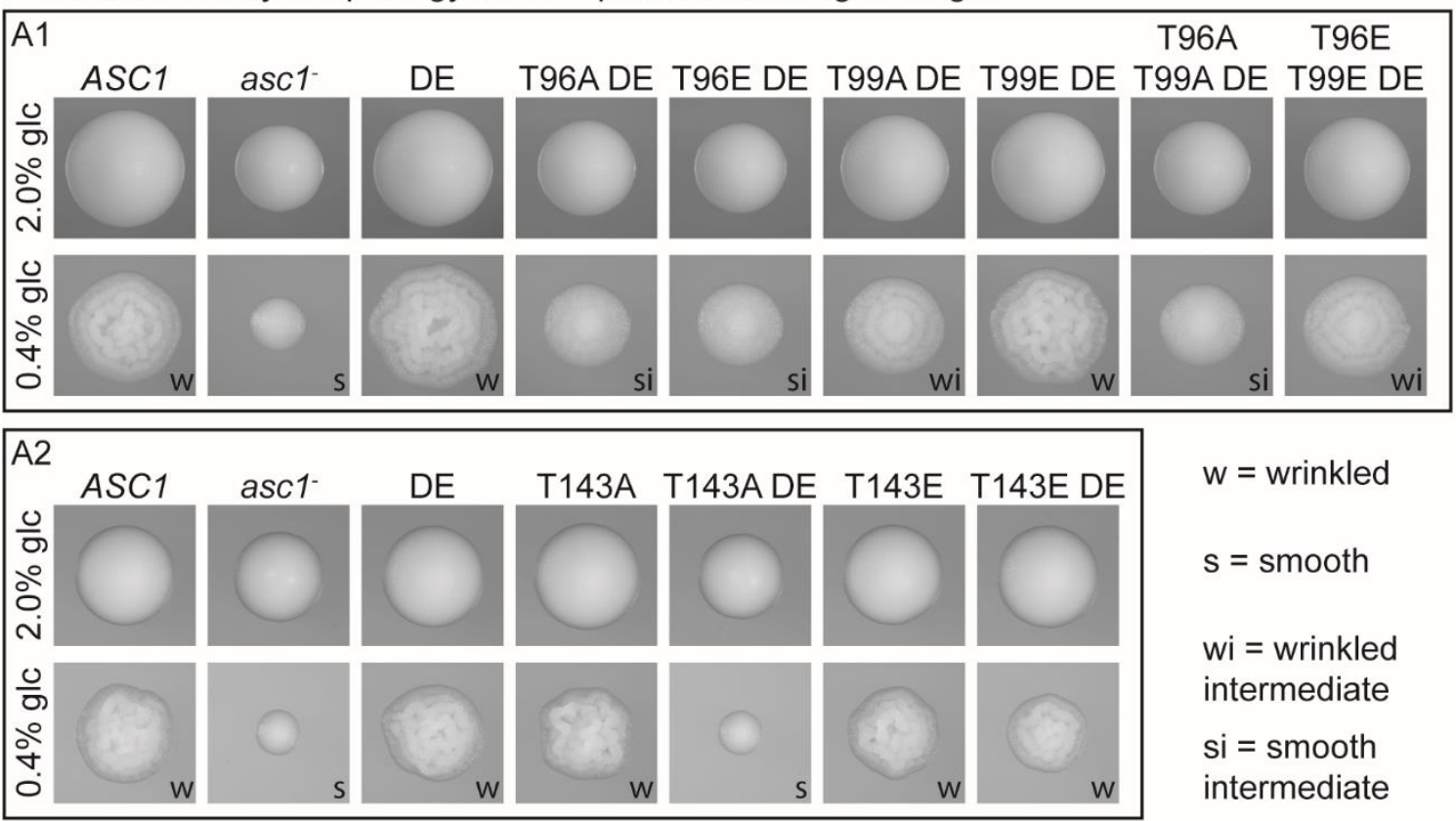

B Colonies grown on YNB plates with $0.4 \%$ glc and overlaid with top-agar including $0.5 \%$ TTC:
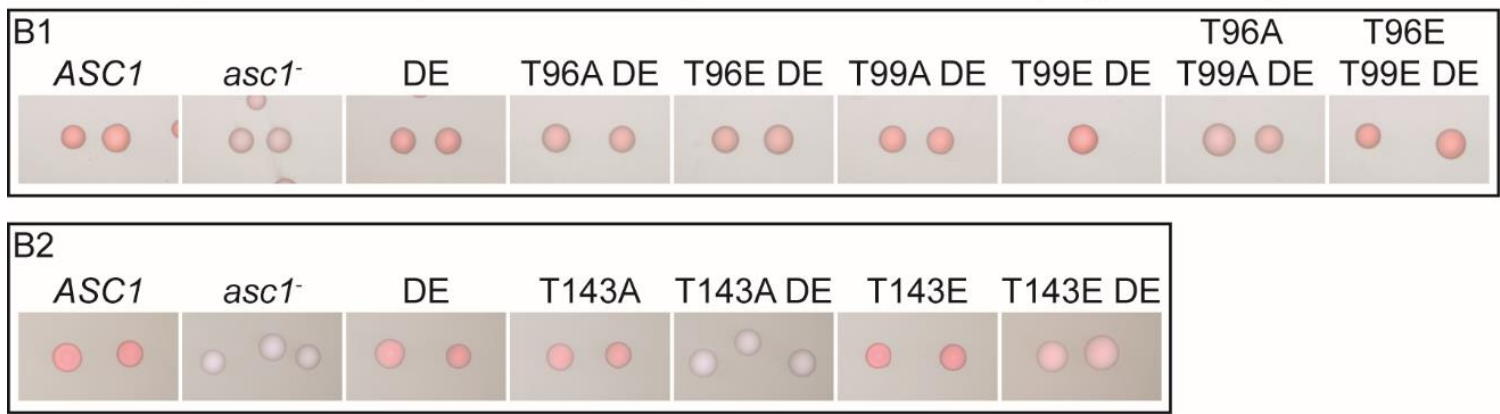

Figure 16. Impact of Asc1p phospho-site mutations on colony morphology and respiratory activity.

(A) Colony morphology under glucose limitation. Cells were grown on YEP 2\% glucose plates (no limitation) and YEP $0.4 \%$ glucose plates (glucose limitation). (B) TTC assay to evaluate respiratory activity. Cells were grown on $0.4 \%$ glucose containing YNB plates. Colonies were overlaid with $1.2 \%$ top agar containing $0.5 \% \mathrm{TTC}$ and incubated for 20 to $30 \mathrm{~min}$ at $30{ }^{\circ} \mathrm{C} .(\mathrm{glc}=$ glucose $)$

by the T96E DE mutation. Combination of the DE mutation with dephosphorylation mimics at S120, S166, and T168 did not affect any of the tested phenotypes (data not shown, substitution to glutamate was not tested). Thus, DE-induced alterations in Asc1p-binding to the ribosome caused phenotypes when combined with specific phospho-site mutations. A high-copy plasmid bearing the wild-type ASC1 gene complemented all mutations and by that revealed a recessive nature of the described ascl mutations (Figure 18). 


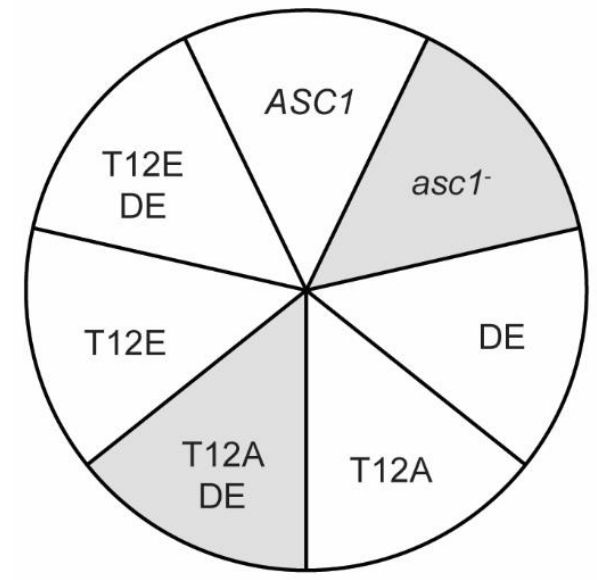

amino acid starvation

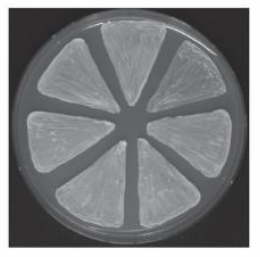

before wash

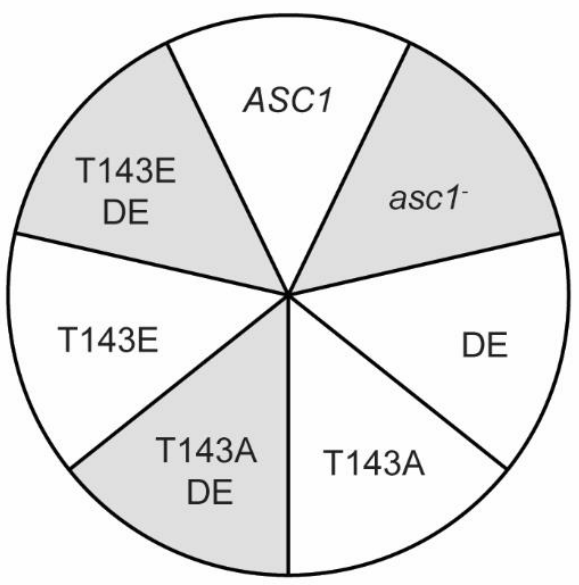

amino acid starvation

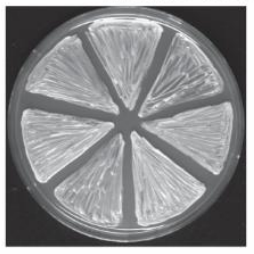

before wash

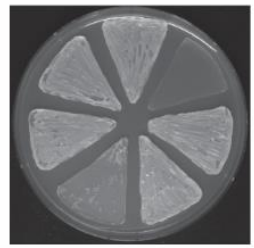

after wash

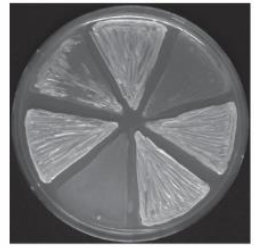

after wash

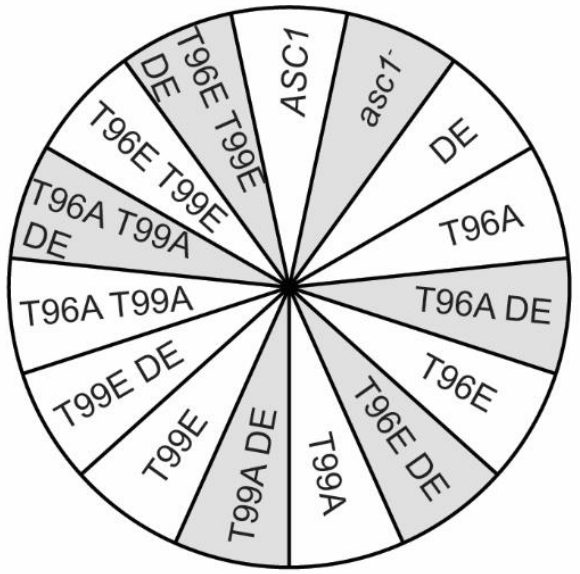

amino acid starvation

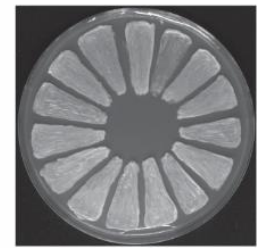

before wash

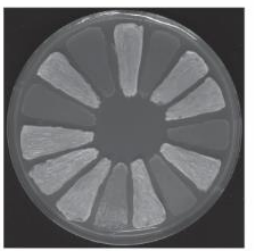

after wash

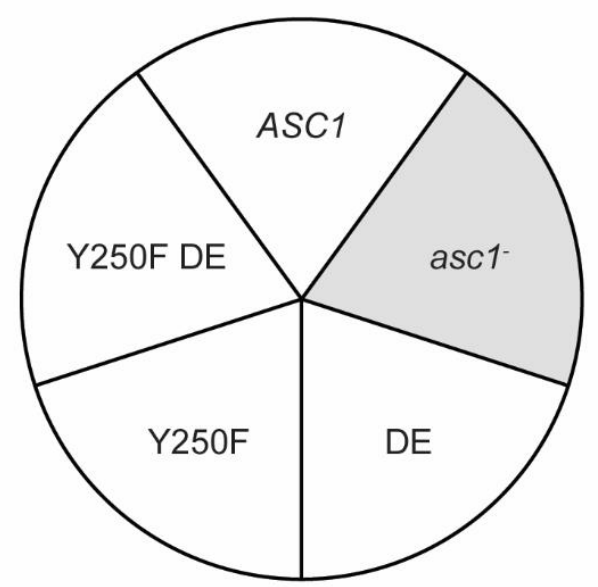

amino acid starvation

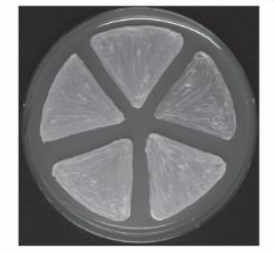

before wash

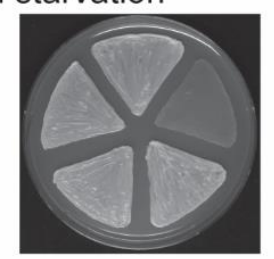

after wash

Figure 17. Test for haploid adhesive growth of phospho-site mutant strains.

Cells were patched on $10 \mathrm{mM} 3$-AT containing YNB plates and incubated for $3 \mathrm{~d}$. Plates are depicted before and after washing with a constant stream of water. 
A

plasmid-borne Asc1p:
\begin{tabular}{|c|c|}
\hline ASC1 & asc1- \\
\hline DE & $\Delta a s c 1$ \\
\hline T12A DE & \\
\hline
\end{tabular}
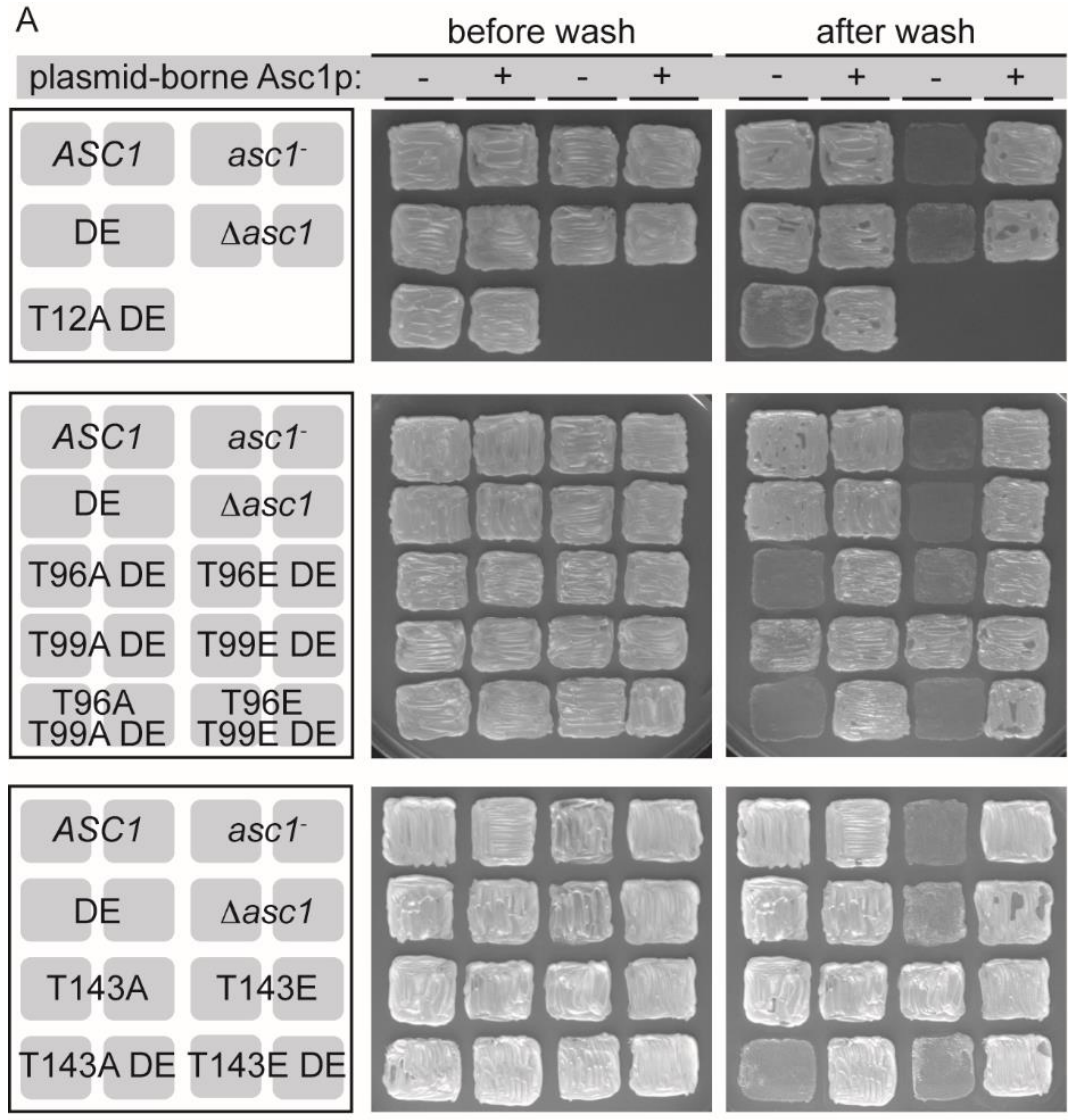

amino acid starvation

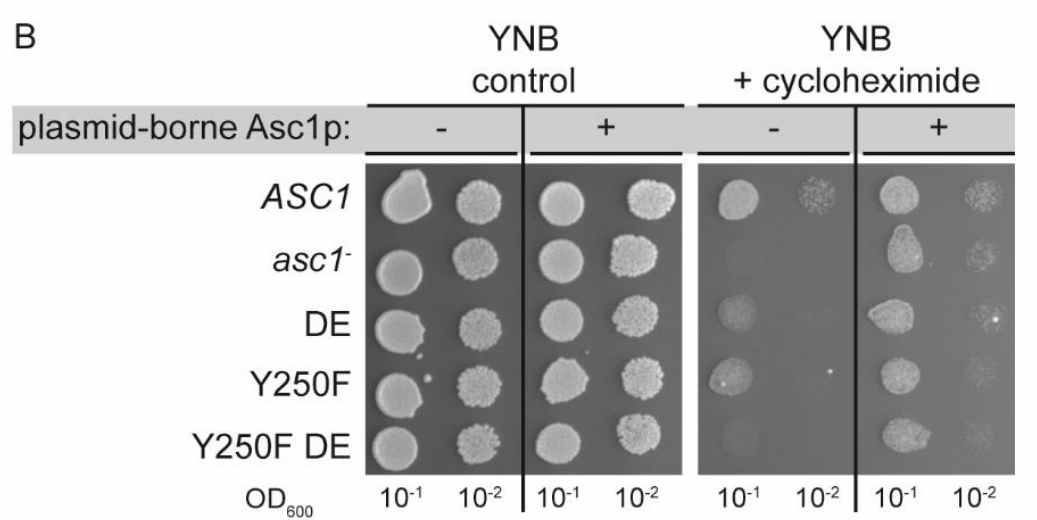

Figure 18. Complementation of $A S C 1$ phospho-site mutant phenotypes by expression of plasmidborne wild-type $A S C 1$.

Phospho-site mutants were transformed with a high-copy number plasmid carrying the wild-type $A S C 1$ gene under the control of the MET25-promoter (ASC1; pME2624) or with the empty vector (EV; pME2787) as control. The ASCl wild-type, ascl, $\triangle a s c 1$, and asclDE strains were transformed with the same plasmids as further controls. (A) Haploid adhesive growth at amino acid starvation caused by $10 \mathrm{mM}$ 3-AT. Plasmid-transformed phospho-site mutants $a s c 1^{\mathrm{T} 12 \mathrm{~A}} \mathrm{DE}$, ascl ${ }^{\mathrm{T} 96 \mathrm{~A}} \mathrm{DE}$, $a s c l^{\mathrm{T} 96 \mathrm{E}} \mathrm{DE}$, $a s c l^{\mathrm{T} 99 \mathrm{~A}} \mathrm{DE}$, $a s c l^{\mathrm{T} 96 \mathrm{~A}}{ }^{799 \mathrm{~A}} \mathrm{DE}$, $a s c l^{\mathrm{T} 96 \mathrm{E}}{ }^{\mathrm{T} 99 \mathrm{E}} \mathrm{DE}$, $a s c l^{\mathrm{T} 143 \mathrm{~A}} \mathrm{DE}$, and $a s c l^{\mathrm{T} 143 \mathrm{E}} \mathrm{DE}$ as well as control strains were patched on the 3-AT containing YNB plates and subjected to wash tests after $3 \mathrm{~d}$ of growth. The $a s c 1^{\mathrm{T} 99 \mathrm{E}} \mathrm{DE}$, ascl ${ }^{\mathrm{T} 143 \mathrm{~A}}$, and $a s c 1^{\mathrm{T} 143 \mathrm{E}}$ strains, which are not impaired in adhesive growth, served as additional controls. Plates are shown before and after the washing step. (B) Drop dilution assay to evaluate cycloheximide sensitivity. Cell suspensions of plasmid-transformed phospho-site mutant strains $\operatorname{ascl}^{\mathrm{Y} 250 \mathrm{~F}}$ and $a s c 1^{\mathrm{Y} 250 \mathrm{~F}} \mathrm{DE}$ as well as control strains were applied onto YNB plates containing $0.15 \mu \mathrm{g} / \mathrm{ml}$ cycloheximide. 
3.2.4 Phosphorylation of T12, T96, T99, and especially T143 is required for cellular abundance of Asc1DEp

The cellular abundance of the Asc1DE protein was found to be rather increased compared to that of Asc1p in the wild-type strain (Figure 19). The abundance of Asc1DEp requires the integrity of the phospho-sites T12, T96, T99, and especially T143, but not Y250. Yet, none of the phospho-site exchanges alone affected the abundance of Asc1p in the absence of the DE mutation (Figure 19 and data not shown). Replacing residue T143 by alanine caused the severest reduction in Asc1DEp abundance even far below the Asc1p levels in wild-type cells. This correlates with the phenotypes of this strain that are reminiscent of those of the asclstrain. The exchange of T143 by a phosphorylation-mimicking glutamate residue reduced the Asc1DEp levels, yet, to a lesser extent.

The combination of DE with T96 replaced either by alanine or by glutamate caused a severe reduction of Asc1p abundance below wild-type levels as well. This effect was enhanced for the T96 alanine substitution through the simultaneous exchange of its neighboring amino acid T99 to alanine in strain $a s c 1^{\mathrm{T} 96 \mathrm{~A}}{ }^{\mathrm{T} 99 \mathrm{~A}} \mathrm{DE}$. In contrast, the $a s c 1^{\mathrm{T} 96 \mathrm{E}}{ }^{199 \mathrm{E}} \mathrm{DE}$ strain exhibited

A

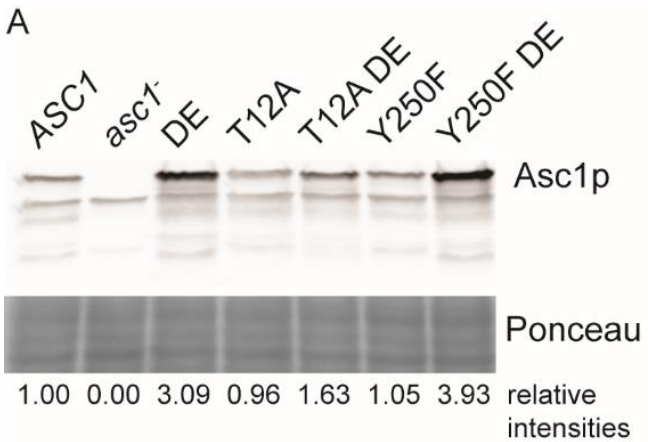

B

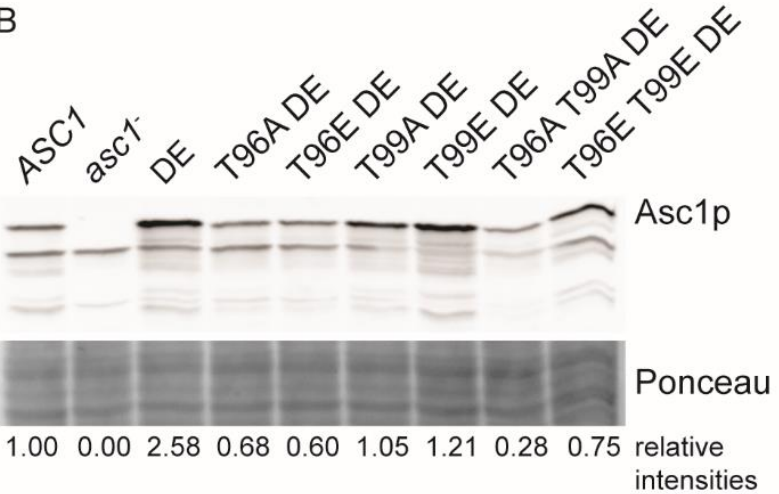

C
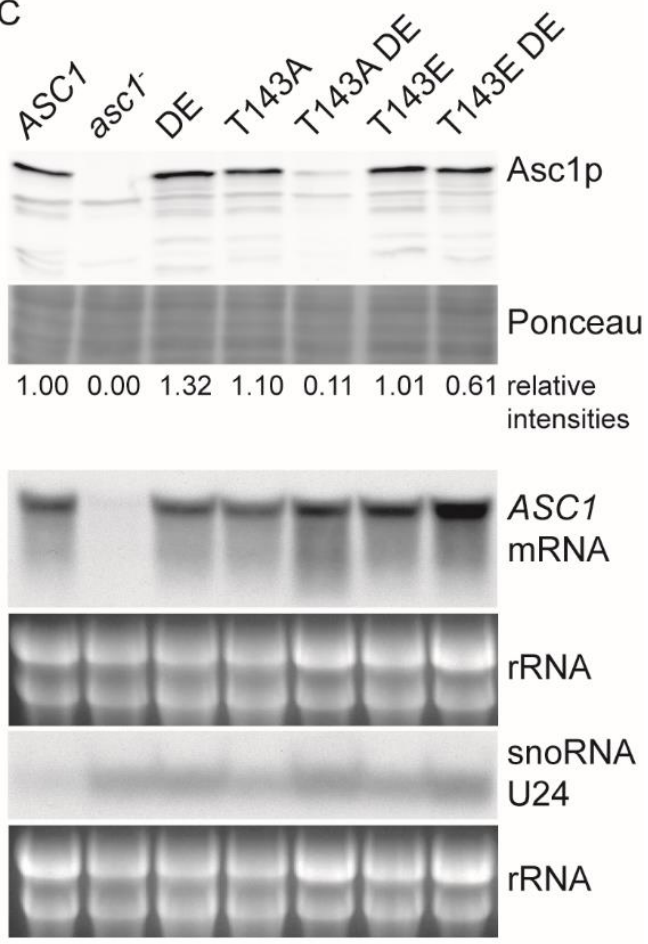

Figure 19. Asc1 protein abundance in phospho-site mutant strains.

(A-C top) Immunodetection of Asc1p within cell extracts derived from ASC1 wild-type, asc1DE, and phospho-site mutant strains and the asc $^{-}$strain as negative control. Ponceau red staining of proteins served as loading control, and a part of the lower half of the stained Western blot membrane is depicted. Fold changes relative to wild-type signal intensities are given below each lane. (C bottom) Northern blot analyses for detection of ASC1 mRNA and SNR24 snoRNA U24 levels in T143 phospho-site mutant strains. rRNA served as loading control. 
higher levels of Asc1p, an observation that is also in line with the phenotypes of this strain. Remarkably, the T96E T99E exchanges rescued the sensitivity of the asclDE strain to cycloheximide independently of Asc1p levels in the $a s c 1^{\mathrm{T} 96 \mathrm{E}}{ }^{999} \mathrm{E} \mathrm{DE}$ strain that are reduced in comparison to the asc1DE strain.

The asc1 ${ }^{\mathrm{Y} 250 \mathrm{~F}} \mathrm{DE}$ strain displayed Asc1p levels similar to the asclDE strain. Thus, the increased sensitivity of both the $a s c 1^{\mathrm{Y} 250 \mathrm{~F}}$ and the $a s c 1^{\mathrm{Y} 250 \mathrm{~F}} \mathrm{DE}$ strain to cycloheximide and canavanine cannot be explained by the cellular abundance of Asc1p in these strains. To exclude that the changes in Asc1p expression were caused at the ASC1 mRNA level, Northern blot experiments were performed with a probe against exon 1 of $A S C 1$. The $a s c 1^{\mathrm{T} 143 \mathrm{~A}} \mathrm{DE}$ strain with the strongest reduction of Asc1p abundance revealed unchanged levels of ASC1 mRNA suggesting that the reduced Asc1p-levels were caused post-transcriptionally either through mRNA translation or protein stability (Figure 19C).

\subsection{The Asc1p-dependent phospho-proteome}

The evidence for Asc1p-phosphorylation and the knowledge about Asc1p being an important player in cellular signal transduction prompted us to investigate Asc1p-dependent proteinphosphorylation beyond its own phosphorylation. Previous studies reported that the absence of Asc1p leads to increased phosphorylation of the MAPKs Kss1p and Slt2p, which control filamentous growth, mating, and cell wall integrity (Chasse et al., 2006; Zeller et al., 2007). Here, the phosphorylation of the MAPK of the high osmolarity response pathway, Hog1p, was analyzed using an antibody for the protein phosphorylated at residues T174 and/or Y176 by its upstream MAP2K, Pbs2p. A significant decrease in Hog1p phosphorylation was detected in the ascl $^{-}$strain, but not in the asclDE strain (Figure 20A). This might explain the sensitivity of the ascl $^{-}$strain to osmotic stress (Figure 20B, Melamed et al., 2010).
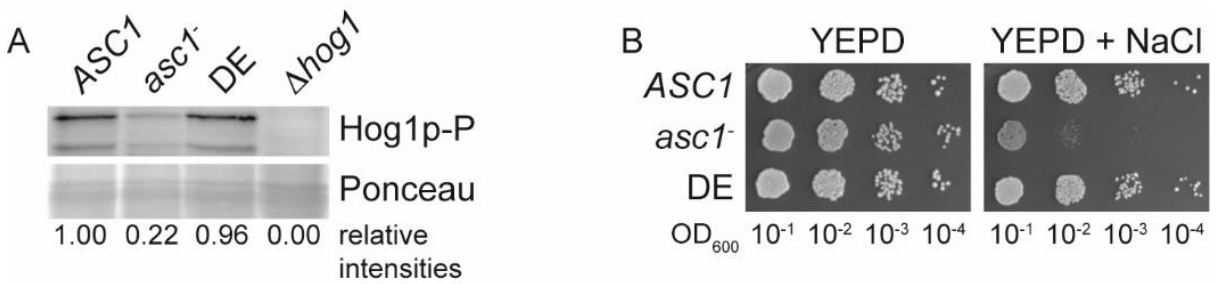

Figure 20. Asc1p-dependency of the high osmolarity response pathway.

(A) Immunodetection of Hog1p phosphorylated at T174 and/or Y176 within cell extracts obtained from $A S C 1$ wild-type, asc $1^{-}$, and asclDE cells using a phospho-p38 MAP kinase-specific antibody. As loading control, proteins were stained with Ponceau red, and a part of the stained Western blot membrane approximately at the level of the Hog1p-P signals is depicted. Fold changes relative to wild-type signal intensities are given below each lane. (B) Drop dilution assay on YEPD plates containing $75 \mathrm{mM} \mathrm{NaCl}$ to evaluate osmotic stress sensitivity. 


\subsubsection{Asc1p affects the phosphorylation of more than 200 proteins at almost 300 different sites}

To get an unbiased and comprehensive view on Asc1p-dependent phosphorylation of other proteins besides MAPKs, a LC-MS-based quantitative phospho-proteome analyses was performed using stable isotope labeling with amino acids in cell culture (SILAC). The proteomes of the following strains were quantitatively compared to that of the wild-type ASC1 strain: 1.) The $\mathrm{sscl}^{-}$strain, to study the impact of the absence of Asc1p, 2.) the asclDE strain, to observe the consequences of a displaced Asc1p at the ribosome, 3.) the $\operatorname{ascl}^{\mathrm{T} 143 \mathrm{~A}}$, $\operatorname{asc} 1^{\mathrm{T} 143 \mathrm{E}}$, and $a s c 1^{\mathrm{T} 143 \mathrm{E}} \mathrm{DE}$ strains, to investigate the impact of the phospho-site $\mathrm{T} 143$ in the presence and absence of the DE mutation. Prior to the quantitative phospho-proteome analysis that requires the enrichment of phospho-peptides from overall peptide-samples, the total proteomes were quantitatively compared to analyze the overall changes in protein abundance. The total proteome data were used to correlate changes in phosphorylation to changes in protein abundance enabling correction of the phospho-peptide SILAC-ratios with the corresponding protein ratios. For efficient incorporation of the isotopically labeled arginine and lysine, $\triangle \arg 4 \Delta$ lys 1 strains were generated for the ASCl wild-type, the ascl-, and the asc1DE background. Strains with the phospho-site T143 mutated were arginine and lysine prototroph and therefore cultivated in the presence of the naturally occurring light amino acids $\left(\operatorname{Arg}^{0}, \operatorname{Lys}^{0}\right)$. To distinguish between changes caused by the Asc1p mutations or differences in arginine and lysine metabolism (prototroph ARG4 LYS1 strains versus auxotroph $\Delta a r g 4 \Delta l y s 1$ strains), an auxotrophy-control $A S C 1^{\text {Aux }}$ was included in the experiments that is an ASC1 wild-type strain prototrophic for arginine and lysine (ARG4 LYS1). The triple SILAC approach using light $\left(\mathrm{Arg}^{0}, \mathrm{Lys}^{0}\right)$, medium $\left(\mathrm{Arg}^{6}, \mathrm{Lys}^{4}\right)$, and heavy $\left(\mathrm{Arg}^{10}, \mathrm{Lys}^{8}\right)$ variants of the labels enables the quantitative comparison of these proteomes in one experiment.

The workflow for the peptide sample preparation of the Asc1p-dependent proteome and phospho-proteome analyses is depicted in Figure 21. All experiments included the ASC1 $\Delta a r g 4 \Delta l y s 1$ strain as constant reference for SILAC-ratio reporting. In the following text, the term SILAC-ratio refers to ratios with the respective ASCl $\triangle a r g 4 \Delta l y s 1$ value in the denominator. A detailed description of the LC-MS data evaluation using the MaxQuant/Perseus software is provided in the Materials and Methods section and in Supplementary Tables 2 (proteome) and 3 (phospho-proteome). Briefly, a one-sample $t$-test (p-value < 0.01) was applied for the protein SILAC-ratios of the $\operatorname{ascl}^{-}$and asclDE strains to identify proteins that are significantly regulated. A two-sample $t$-test (p-value $<0.01$ ) was used to assess the difference between the protein SILAC-ratios of the asc $1^{\text {T143 }}$ mutant strains 


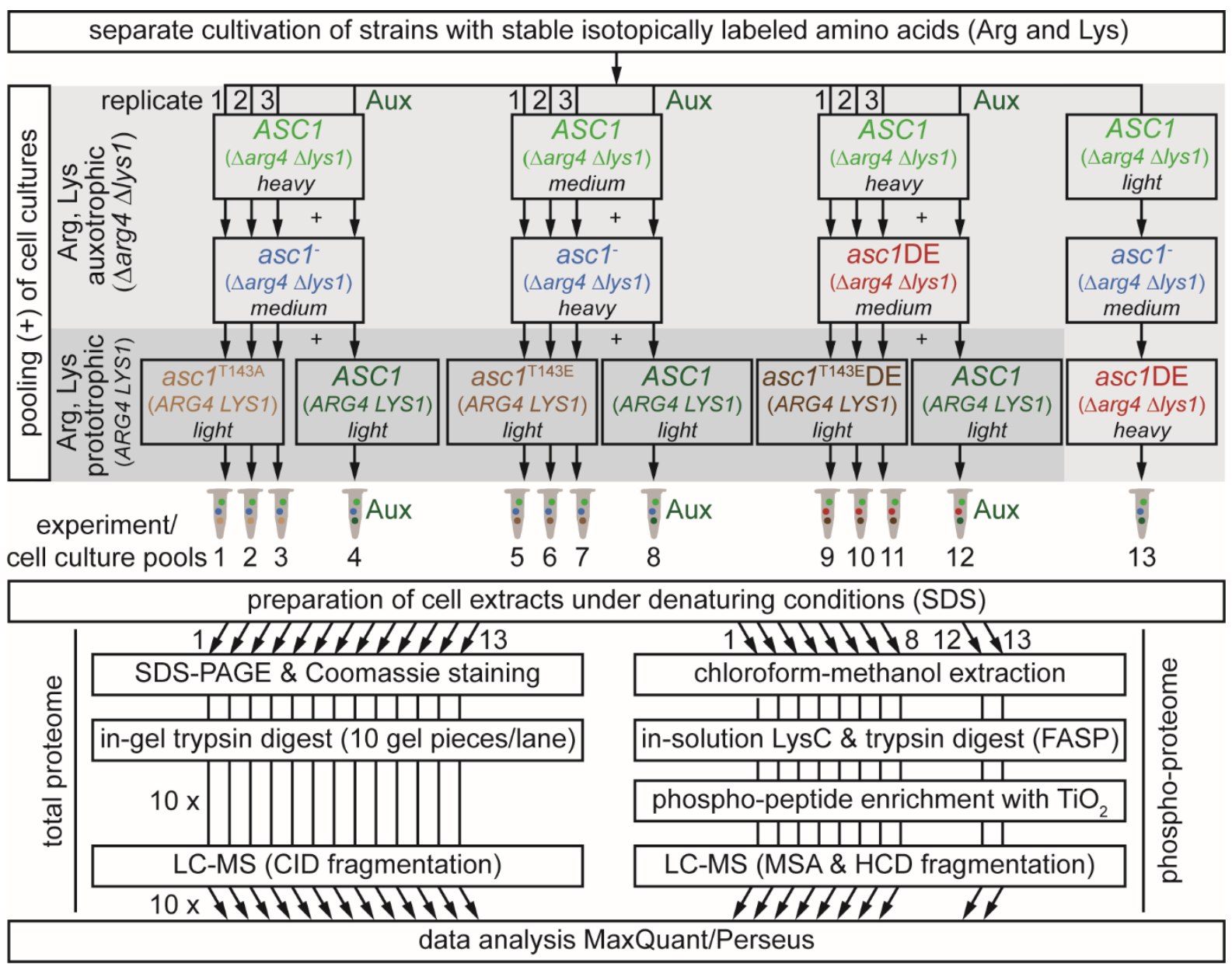

Figure 21. Analysis of the Asc1p-dependent phospho-proteome.

Peptide sample preparation for SILAC and LC-MS-based Asc1p-dependent phospho-proteome and proteome analyses. S. cerevisiae strains were cultivated in the presence of light, medium, or heavy labeled arginine and lysine. Arrows indicate which strains were pooled and indicate the number of replicates. The auxotrophy control (Aux) comprised cells of the ASC1 ARG4 LYSI strain. In total, 13 independent cell pools were obtained and subjected to the subsequent preparation of cell extracts. The protein extracts were split in two and processed separately for the proteome and phospho-proteome analyses. For the proteome analysis, cell extract containing $100 \mu \mathrm{g}$ protein was separated by SDSPAGE, followed by staining with Colloidal Coomassie and in-gel trypsin digestion. For the phosphoproteome analysis, only protein extracts of experiments $1-8$ and 12-13 were further processes. $1 \mathrm{mg}$ protein of each sample was subjected to chloroform-methanol extraction to remove SDS from the samples followed by in-solution digestion (filter-aided sample preparation, FASP, Wiśniewski et al., 2009) with LysC and trypsin. Phospho-peptides were enriched using $\mathrm{TiO}_{2}$ affinity chromatography. The peptide samples were analyzed individually by LC-MS using appropriate fragmentation techniques (CID, MSA-CID, or HCD). All raw data files were searched in one set against an S. cerevisiae-specific protein database with the MaxQuant software. The downstream data evaluation was performed with Perseus as described in Supplementary Tables 2 and 3.

and the corresponding values of the auxotrophy controls. In the $\mathrm{ascl}^{-}$strain, 604 proteins were significantly affected in their abundance with an averaged $\log _{2}$ SILAC-ratio $<-0.26$ or $>0.26$ (Supplementary Table 4). Using this threshold for regulation twelve proteins for the asclDE, one and four for the $a s c 1^{\mathrm{T} 143 \mathrm{~A}}$ and $a s c 1^{\mathrm{T} 143 \mathrm{E}}$ strains, respectively, and 111 for the $a s c 1^{\mathrm{T} 143 \mathrm{E}} \mathrm{DE}$ 
strain were identified as regulated (Supplementary Tables 5-8). The rather marginal changes in the asclDE, ascl ${ }^{\mathrm{T} 143 \mathrm{~A}}$, and $a s c 1^{\mathrm{T} 143 \mathrm{E}}$ proteomes reflect the observation that these strains behaved phenotypically mainly like the wild-type strain in our tests. Accordingly, 88 of the 111 proteins affected in their abundance by the T143E DE mutation were identified as regulated in the $a s c 1^{-}$strain as well, and in line with this, the $\operatorname{asc} 1^{\mathrm{T} 143 \mathrm{E}} \mathrm{DE}$ strain mostly showed similar or equal phenotypes as the Asc1p-deficient strain.

Based on these observations, the phospho-proteome analysis focused on the asc $1^{-}$strain. The respective peptide samples were subjected to the enrichment of phospho-peptides and LC-MS analysis. In total, 1947 distinct phospho-sites (localization probability $\geq 0.5$ ) were identified. 1245 of these sites were detected and quantified in at least two independent samples for the comparison between the asc $1^{-}$and ASC1 wild-type strain and considered for further analysis. A two-sample $t$-test (p-value < 0.01) was applied comparing the SILAC-ratios of the phospho-sites with those of the corresponding proteins. The significantly regulated phosphosites were further filtered for their degree of regulation considering only sites with a normalized median $\log _{2}$ SILAC-ratio $<-0.26$ or $>0.26$ (Supplementary Table 9). Table 4 lists phospho-sites with an even stronger regulation of median $\log _{2}$ SILAC-ratios $\leq-0.58$ or $\geq 0.58$. Phospho-sites with zero to two quantification values for the corresponding protein were also considered applying a one-sample $t$-test on their SILAC-ratios. In total, 289 phospho-sites in 208 proteins showed Asc1p-dependent changes in their degree of phosphorylation. 139 of these sites were found to be up-regulated and 151 down-regulated including one site (S222 of Vid27p) that was identified as up-regulated for the singly phosphorylated peptide and downregulated for the doubly phosphorylated peptide (T220 and S222). Six other sites were also identified twice within singly and doubly phosphorylated peptides, but showed the same direction of regulation. 62 proteins were identified with more than one Asc1p-dependently regulated phospho-site, and eight of these proteins contained sites that were increased and others that were decreased in their degree of phosphorylation.

Table 4. Asc1p-dependently regulated phospho-sites.

All proteins containing phospho-sites with a proteome-corrected median $\log _{2}$ asc1-/ASC1 SILAC-ratio $\leq-0.58$ or $\geq 0.58$ are listed together with their regulated sites and the corresponding localization probabilities (site \& prob). The colors reflect the values of the median SILAC-ratios for the phosphosite (phos), the corresponding protein (prot), and the proteome-correction ( $\log _{2}$ ratio phospho-site minus $\log _{2}$ ratio protein, named phospho regulation). Gray indicates that the protein was not quantified in the total proteome analysis. In this case, the phospho regulation is identical with the quantification value for the phospho-site. 1 indicates that only one quantification value was obtained. Phospho-sites that are printed in italics are previously unknown sites according to PhosphoGRID (http://www.phosphogrid.org, Stark et al., 2006) and SGD including data from Swaney et al. (2013). See Supplementary Table 9 for details and additional phospho-sites with a proteome-corrected $\log _{2}$ asc $1^{-} /$ASC1 SILAC-ratio $<-0.26$ or $>0.26$. 
Table 4.

\begin{tabular}{|c|c|c|c|c|c|}
\hline \multirow[b]{2}{*}{ protein } & \multirow{2}{*}{\multicolumn{2}{|c|}{ site \& prob }} & \multicolumn{2}{|c|}{$\begin{array}{l}\text { asc1/ } \\
\text { ASC1 }\end{array}$} & \multirow{2}{*}{ 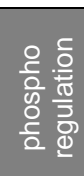 } \\
\hline & & & $\begin{array}{l}0 \\
0 \\
-\frac{1}{0}\end{array}$ & 힘 & \\
\hline Edc1 & S82 0. & 0.99 & & & 2.15 \\
\hline Tpi1 & $\begin{array}{ll}\mathrm{S} 215 & 1 .\end{array}$ & 1.00 & & & 1.47 \\
\hline Csr1 & S2 1. & 1.00 & & & 1.45 \\
\hline Pre8 & $S 151$. & 1.00 & & & 1.16 \\
\hline Rpn7 & $\begin{array}{ll}S 196 \quad 1 . \\
\end{array}$ & 1.00 & & & 1.11 \\
\hline Isc1 & $\begin{array}{ll}\text { T361 } & 1 .\end{array}$ & 1.00 & & & 0.95 \\
\hline Rpl12a/b & $\begin{array}{ll}\text { S38 } & 1 .\end{array}$ & 1.00 & & & 0.93 \\
\hline Ren1 & $\mathrm{S} 117 \quad 1$. & 1.00 & & & 0.91 \\
\hline Ren1 & $\begin{array}{ll}\mathrm{S} 113 & 1 .\end{array}$ & 1.00 & & & 0.91 \\
\hline Ste20 & T572 0. & 0.61 & & & 0.90 \\
\hline Pin4 & S194 1. & 1.00 & & & 0.87 \\
\hline Nte1 & S634 0. & 0.99 & & & 0.83 \\
\hline Ste20 & $\begin{array}{ll}\text { S169 } 1 . \\
\text {. }\end{array}$ & 1.00 & & & 0.82 \\
\hline Ty2a* & S424 0. & 0.99 & & & 0.81 \\
\hline $\operatorname{Imh} 1$ & T304 0. & 0.65 & & & 0.81 \\
\hline Acm1 & $\begin{array}{ll}\text { S48 } 1 .\end{array}$ & 1.00 & & & 0.80 \\
\hline Aim3 & S843 0. & 0.93 & & & 0.79 \\
\hline $\operatorname{Imh} 1$ & S308 0. & 0.98 & & & 0.72 \\
\hline Smi1 & $\begin{array}{ll}\text { S389 } & 0 .\end{array}$ & 0.92 & & & 0.72 \\
\hline Nnk1 & $\begin{array}{ll}739 & 1 .\end{array}$ & 1.00 & & & 0.70 \\
\hline Iml2 & S7 1. & 1.00 & & & 0.70 \\
\hline Pgm3 & T156 0. & 0.66 & & & 0.69 \\
\hline Kri1 & Y482 0. & 0.63 & & & 0.68 \\
\hline Rlp7 & $\begin{array}{ll}\mathrm{T} 120 & 1 . \\
\end{array}$ & 1.00 & & & 0.67 \\
\hline Rpn1 & S19 1. & 1.00 & & & 0.67 \\
\hline Tif1 & S2 1. & 1.00 & & & 0.67 \\
\hline Acc1 & s9 0. & 0.87 & & & 0.66 \\
\hline Fyv8 & S441 0. & 0.99 & & & 0.65 \\
\hline Dig2 & $\begin{array}{ll}\text { T83 } & 0 .\end{array}$ & 0.76 & & & 0.64 \\
\hline Rpo21 & $\begin{array}{ll}\text { T1471 } & 0 .\end{array}$ & 0.93 & & & 0.64 \\
\hline Pgm3 & S158 0. & 0.97 & & & 0.64 \\
\hline $\operatorname{Imh} 1$ & S827 0. & 0.52 & & & 0.64 \\
\hline Ste20 & S502 1. & 1.00 & & & 0.63 \\
\hline Rrp36 & S14 1. & 1.00 & & & 0.63 \\
\hline Myo3 & $\begin{array}{ll}\text { S357 } & 1 .\end{array}$ & 1.00 & & & 0.62 \\
\hline Pda1 & $\begin{array}{ll}\mathrm{S} 313 & 1 .\end{array}$ & 1.00 & & & 0.62 \\
\hline Dig2 & T82 0. & 0.94 & & & 0.62 \\
\hline Pdr16 & S349 0. & 0.69 & & & 0.61 \\
\hline Pdr16 & S346 0. & 0.97 & & & 0.61 \\
\hline Stb1 & T99 1. & 1.00 & & & 0.60 \\
\hline Stb1 & S72 1. & 1.00 & & & 0.58 \\
\hline Ppq1 & $\mathrm{S} 2080$. & 0.89 & & & 0.58 \\
\hline Ysc84 & $\begin{array}{ll}\text { S301 } & 1 .\end{array}$ & 1.00 & & & 0.58 \\
\hline
\end{tabular}

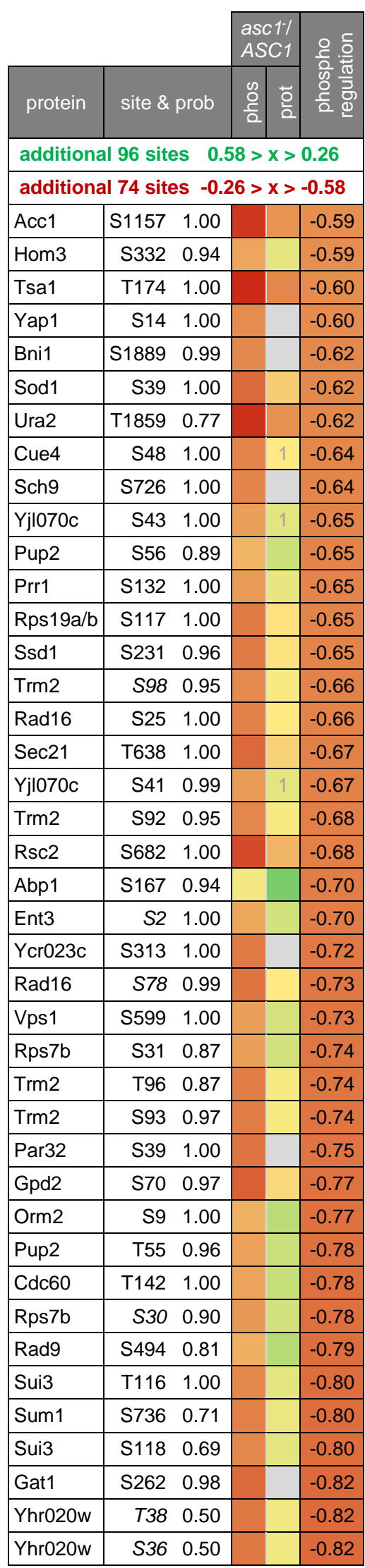

\begin{tabular}{|c|c|c|c|c|c|}
\hline & & & & & \\
\hline & & & $A S C$ & & 은 들 \\
\hline protein & site \& p & prob & $\begin{array}{l}0 \\
0 \\
0 \\
0\end{array}$ & to & 응 $\overline{\frac{5}{2}}$ \\
\hline Zeo1 & T49 & 1.00 & & & -0.83 \\
\hline Zeo1 & $\mathrm{S} 40$ & 1.00 & & & -0.83 \\
\hline Gcs1 & S157 & 0.81 & & & -0.85 \\
\hline Ura2 & S1857 & 0.98 & & & -0.86 \\
\hline Ty1b** & S1004 & 0.67 & & & -0.86 \\
\hline Kin2 & S549 & 1.00 & & 1 & -0.87 \\
\hline Vid27 & T220 & 1.00 & & & -0.94 \\
\hline Vid27 & S222 & 1.00 & & & -0.94 \\
\hline Def1 & T258 & 0.90 & & & -0.95 \\
\hline Meh1 & S146 & 0.99 & & & -0.95 \\
\hline Vtc3 & S198 & 1.00 & & & -0.99 \\
\hline Tdh1/2/3 & S201 & 1.00 & & & -1.01 \\
\hline Gvp36 & S2 & 1.00 & & & -1.03 \\
\hline Rho5 & $\mathrm{S} 223$ & 1.00 & & & -1.03 \\
\hline Abp1 & S169 & 1.00 & & & -1.03 \\
\hline Mnr2 & T177 & 0.99 & & & -1.05 \\
\hline Hsp42 & S223 & 0.99 & & & -1.06 \\
\hline Def1 & S260 & 1.00 & & & -1.08 \\
\hline Nrg2 & S100 & 0.77 & & & -1.08 \\
\hline Kns1 & T562 & 1.00 & & & -1.10 \\
\hline Mnr2 & S152 & 1.00 & & & -1.14 \\
\hline Tif4632 & T196 & 1.00 & & & -1.18 \\
\hline Bre5 & S282 & 1.00 & & & -1.20 \\
\hline Grx2 & S94 & 0.94 & & & -1.30 \\
\hline Ctr9 & S1017 & 1.00 & & & -1.31 \\
\hline Puf3 & S86 & 1.00 & & & -1.34 \\
\hline Ugp1 & $\mathrm{Y} 13$ & 0.55 & & & -1.42 \\
\hline Grx2 & S91 & 1.00 & & & -1.42 \\
\hline Cdc28 & $\mathrm{Y} 19$ & 0.98 & & & -1.46 \\
\hline Abp1 & T181 & 1.00 & & & -1.53 \\
\hline Abp1 & S183 & 1.00 & & & -1.53 \\
\hline Nrg2 & T99 & 0.95 & & & -1.64 \\
\hline Mep2 & $S 460$ & 0.73 & & & -1.67 \\
\hline Mep2 & T459 & 0.63 & & & -1.70 \\
\hline Tpo1 & S72 & 1.00 & & & -1.71 \\
\hline Egd1 & T151 & 1.00 & & & -3.36 \\
\hline
\end{tabular}

${ }^{*}$ Ty2a-Dr3;Ty2a-C;Ty2a-Or1; Ty2aLr2;Ty2a-Gr2;Ty2a-F

**Ty1b-Lr4;Ty1b-Pr1;Ty1b-Gr2;

Ty1b-Pr2;Ty1b-Er1;Ty1b-MI2;

Ty1b-OI; Ty1b-Jr1;Ty1b-A;Ty1b-

Ml1;Ty1b-PI;Ty1b-Lr2,

S445/S1004/S1005

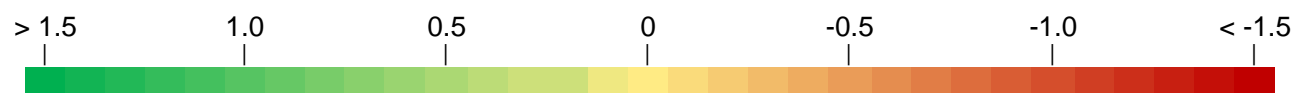


Due to the triple-SILAC approach the analyzed samples also contained information about the $\operatorname{asc} 1^{\mathrm{T} 143 \mathrm{~A}}$ and $a s c 1^{\mathrm{T} 143 \mathrm{E}}$ phospho-proteomes that were further evaluated (see Supplementary Tables 10-11). As expected from the results for the total proteomes, only a few changes were observed in the phospho-proteomes of the T143 mutant strains with nine regulated phosphoproteins. Four of them were already found as regulated in the $a s c 1^{-}$strain (Ent3p, Sec31p, Rps1a/bp, and Tif1p). Phosphorylation of S2 of Tif1p was down-regulated in the ascl $1^{\text {T143E }}$ strain, whereas the modification was increased in $\mathrm{ascl}^{-}$cells, and Sec31p phosphorylation was altered at a different site (S980 instead of S999) in the ascl ${ }^{\mathrm{T} 143 \mathrm{~A}}$ strain. In total, 213 proteins were identified as Asc1p-dependently regulated in their phosphorylation that were further evaluated according to their related biological processes.

\subsubsection{Asc1p spreads signals to fundamental processes of eukaryotic gene expression}

To evaluate which processes are affected by the Asc1p-dependent phosphorylation network, the 213 Asc1p-dependently regulated phospho-proteins were assigned to biological processes (gene ontology term) making use of the Database for Annotation, Visualization and Integrated Discovery (DAVID) $v 6.7$ (Huang da et al., 2009a, b). From this initial computational analysis seven different groups were established, and all but 18 proteins were assigned to these functional categories in a non-exclusive manner (Figure 22A, Supplementary Table 12). One of the largest groups consists of proteins related to mRNA translation. Some of them are directly involved in this process, such as ribosomal proteins (e.g., Rpl12a/bp), translation factors, and mRNA-binding proteins (e.g., Puf3p). Eight translation initiation factors were identified to be regulated comprising the RNA-helicase eIF4A, which was found with increased phosphorylation in the absence of Asc1p in an earlier study of our group (Valerius et al., 2007). Besides initiation factors, this category also comprises the elongation factor Yef3p and the termination factor Sup35p. Egd1p, a component of the nascent polypeptide-associated complex, and Ssz1p, a part of the ribosomeassociated complex, were further shown to be affected in their phosphorylation status. The observed increase in phosphorylation of Ssz1p at residue S480 confirms again an earlier finding of our group (Valerius et al., 2007). Proteins indirectly involved in translation, such as tRNA-modifying enzymes, ribosome biogenesis factors, and tRNA-synthethases, were assigned to the group of translation as well.

The Asc1p-dependent phosphorylation network is connected to further processes that contribute to cellular protein homeostasis including transcription and protein turnover. Absence of Asc1p alters, e.g., phosphorylation of the transcription factor Fhl1p, a key 
A

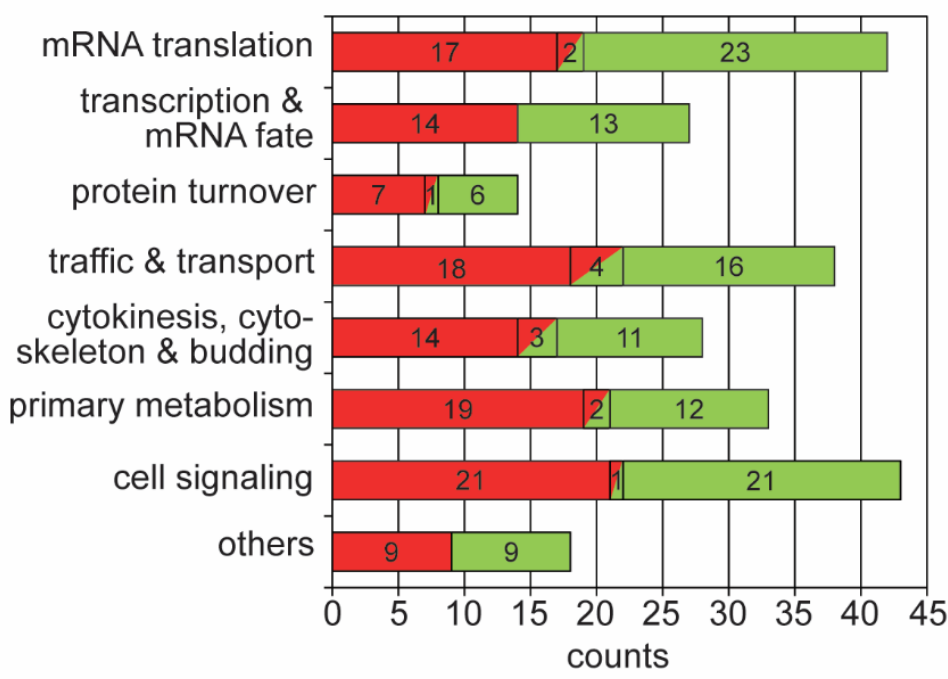

- down-regulated घdown- and up-regulated uup-regulated

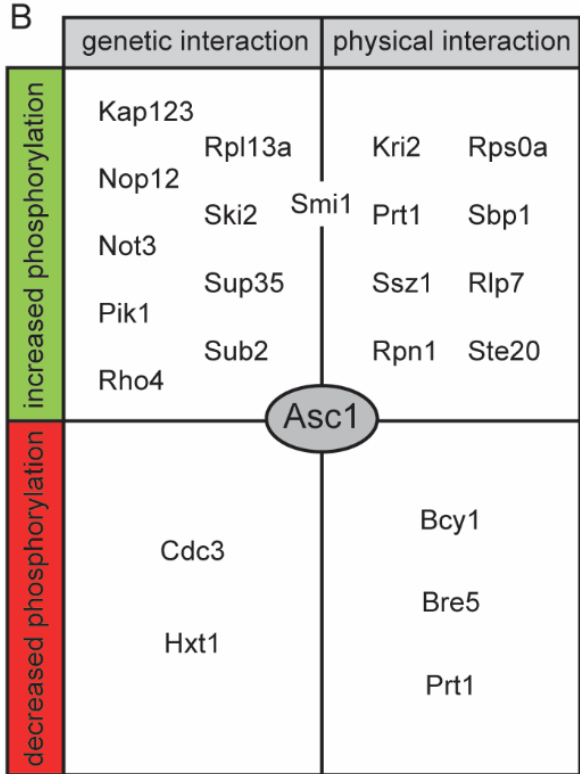
in the asc $1^{-}$strain and/or asc $1^{\top 143}$ mutant strains

Figure 22. Cellular processes and known Asc1p-interaction partners that are targeted by Asc1pdependent phosphorylation.

(A) Assignment of the Asc1p-dependently regulated phospho-proteins to cellular processes. The graph depicts the number of proteins assigned to the different groups (see Supplementary Table 12 for the identity of proteins in each group). The 213 proteins were grouped in a non-exclusive manner meaning that 30 proteins are present in two groups. (B) Known Asc1p-interacting proteins Asc1p-dependently regulated on the level of phosphorylation.

regulator of ribosomal protein encoding genes including ASCl itself (Kleinschmidt et al., 2006). The category transcription was additionally expanded for proteins affecting $m R N A$ fate, such as the decapping protein Edc1. The group protein turnover includes three components of the regulatory particle of the $26 \mathrm{~S}$ proteasome (Rpn1p, Rpn7p, and Rpn13p) and two components of its catalytic core (Pre8p and Pup2p), as well as Bre5p, a deubiquitination cofactor of Ubp3p, which regulate bulk protein degradation during ribophagy and mitophagy (Müller et al., 2015). The group traffic and transport comprises central structural components of vesicle coats (e.g., Sec21p) and regulators of vesicle-mediated transport (e.g., Gcs1p). Also, transmembrane transporters, such as the polyamine transporter Tpo1p and the ammonium transporter Mep2p, were assigned to this category. Asc1p affects the phosphorylation of several proteins that are related to cytokinesis, cytoskeleton organization, and budding, for instance, proteins involved in actin patch assembly at sites of polarized growth (Sla2p and Ent1p), polarisome components (Spa2p and Bni1p), actin nucleation promoting factors (Myo3/5p and Abp1p), regulators of actin filament elongation (Aim3p and Abp1p), and profilin (Pfy1p). Another group comprises enzymes of the primary metabolism that are, e.g., involved in amino acid biosynthesis (e.g., Hom3p), glycolysis and gluconeogenesis (e.g., Tpi1p and Tdh1/2/3p), and pyrimidine biosynthesis (Ura2p). 
Finally, the largest group contains proteins implicated in cell signaling. This group stands in close relation to all other groups since it comprises 14 protein kinases, such as Cdc28p, Sch9p, and Ste20p, and regulatory subunits of the protein phosphatase Glc7p (Glc8p and Ypi1p) that could be directly responsible for the observed Asc1p-dependent changes in the phospho-proteome. In summary, the proteome data reveal an unprecedented strong impact of a single WD40 repeat protein on the phosphorylation of proteins involved in the regulation and realization of fundamental processes in a eukaryotic cell. Figure 22B shows proteins that were identified here with Asc1p-sensitive phospho-sites and were found in previous studies to genetically or physically interact with Asc1p. The physical interaction partners are potential candidates for an Asc1p-mediated kinase/phosphatase-target interaction.

\subsection{Identification of Asc1p-interacting proteins}

The results presented in the previous chapter showed a severe impact of Asc1p on the S. cerevisiae phospho-proteome. As a scaffold protein, Asc1p is supposed to provide proximity of proteins, and it might localize kinases or phosphatases to their substrates. Mammalian RACK1 was shown to contribute to the recruitment of kinases to the ribosome for the subsequent phosphorylation of translation initiation factors (Ceci et al., 2003; Ruan et al., 2012). Considering a similar function for Asc1p in yeast, the protein was purified from protein extracts to identify co-purifying proteins. C-terminally Strep-tagged Asc1p was affinity captured via Strep-Tactin ${ }^{\circledR}$ columns followed by LC-MS-based identification of proteins. Also, Asc1DEp-Strep with the two amino acid exchanges R38D and K40E was enriched via Strep-Tactin ${ }^{\circledR}$. This variant is supposed to be compromised in its association to the ribosome and might lead to the identification of interactions that are specific for the mutated protein (Sengupta et al., 2004; Coyle et al., 2009).

In general, the analysis of eluate fractions derived from affinity purification or pull-down experiments with LC-MS results in the identification of hundreds of proteins as unspecific background due to the high sensitivity of modern mass spectrometers, such as the LTQ Orbitrap Velos Pro used in this study. Thus, interaction partners of a bait protein might also be identified with high confidence from negative controls although with lower abundance. In this study, a strain expressing untagged Asc1p was used for the negative control. Reliable quantification methods are essential to compare the abundance of each identified protein between the sample enriched for the bait protein and the negative control. Here, a SILACbased approach was applied for relative peptide and thus protein quantification as described before (chapter 3.3.1). SILAC-labeling allows the pooling of differentially labeled cell 
$\Delta$ asc1 $\Delta$ arg $4 \Delta$ lys $1+$ plasmid $(2 \mu \mathrm{m}$, MET25 promoter $)$ expressing the following ASC1 alleles:

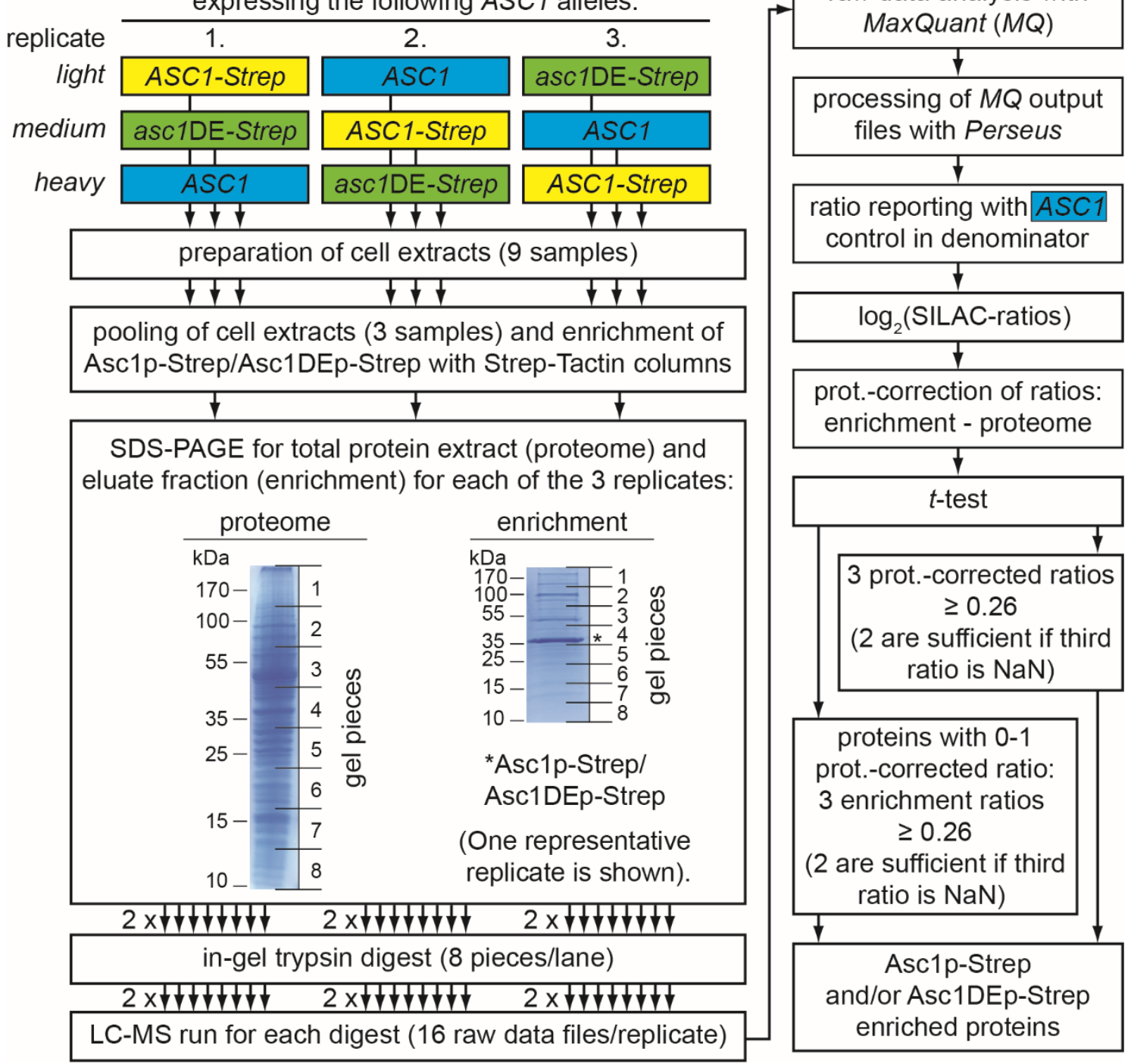

Figure 23. Workflow for the identification of putative interaction partners of Asc1p-Strep and Asc1DEp-Strep.

On the left-hand side of the figure the experimental workflow is illustrated. The experiment was performed with three biological replicates. In each replicate, the cells of three different independently cultured strains were analyzed expressing either Asc1p-Strep, Asc1DEp-Strep, or the untagged Asc1p. For each experiment, the light, medium, and heavy amino acid labels were swapped between the different strains. The steps of cultivation and preparation of cell extracts were performed individually for each sample to prevent the interchange of proteins bound to Asc1p-Strep or Asc1DEp-Strep. The three cell extracts of one replicate were mixed immediately before capturing of Asc1p-Strep and Asc1DEp-Strep with Strep-Tactin ${ }^{\circledR}$ spin columns. The eluate fractions and aliquots of the pooled protein extracts were subjected to SDS-PAGE. A representative SDS-gel after staining of proteins with Colloidal Coomassie is depicted. Each lane was cut into eight gel pieces as indicated in the figure with black lines, and proteins were in-gel digested with trypsin followed by LC-MS analysis of the peptides. In total, 16 LC-MS runs were performed for each biological replicate: 8 for the proteome plus 8 for the enrichment. Raw-data files were searched against an S. cerevisiae-specific database using the MaxQuant software. On the right-hand side of the figure, an overview of the most important steps of the downstream data evaluation with the Perseus software is provided. The pathway splits up into two branches: one for the analysis of proteins identified in the eluate fraction with two or three proteome (prot.)-corrected SILAC-ratios available and a second for the analysis of proteins with no or one proteome-corrected SILAC-ratio. NaN is the abbreviation for not a number and states that the protein was not identified or not quantified in one of the replicates. 
Table 5. Logarithmized SILAC-ratios of proteins co-captured with Asc1p-Strep.

The listed proteins were identified to be enriched together with Strep-tagged Asclp based on their relative quantification using SILAC. The top part lists proteins with 2-3 proteome-corrected SILACratios and the bottom part those with 0-1 proteome-corrected SILAC-ratios. The colors reflect the SILAC-ratios according to the scale below the table. (Abbreviations/symbols: $* * * t$-test $\mathrm{p}$-value $<0.05, *=t$-test $\mathrm{p}$-value $<0.1, \varnothing=$ mean of respective SILAC-ratios, SD $=$ standard deviation, $\mathrm{DE}=$ mean Asc1DEp-Strep/control SILAC-ratios). The last column of the upper part of the table gives the mean and SD of the proteome-corrected Asc1DEp-Strep/control ratios. The last column of the bottom part gives the mean and SD of the enrichment Asc1DEp-Strep/control ratios.

\begin{tabular}{|c|c|c|c|c|c|c|c|c|c|c|c|c|c|}
\hline \multicolumn{14}{|c|}{ 2-3 proteome-corrected SILAC-ratios $\geq 0.26$} \\
\hline & \multicolumn{4}{|c|}{$\begin{array}{l}\text { proteome-corrected } \\
\text { ratios }\end{array}$} & \multicolumn{4}{|c|}{ enrichment ratios } & \multicolumn{4}{|c|}{ proteome ratios } & \multirow{2}{*}{$\begin{array}{c}\mathrm{DE} \\
\varnothing \\
(\mathrm{SD})\end{array}$} \\
\hline protein & $\begin{array}{c}\varnothing \\
(\mathrm{SD}) \\
\end{array}$ & 1 & 2 & 3 & $\begin{array}{c}\varnothing \\
(\mathrm{SD}) \\
\end{array}$ & 1 & 2 & 3 & $\begin{array}{l}\varnothing \\
(\mathrm{SD}) \\
\end{array}$ & 1 & 2 & 3 & \\
\hline $\operatorname{Asc}^{* *}$ & \begin{tabular}{|l|}
7.41 \\
$(1.00)$
\end{tabular} & 7.66 & 8.27 & 6.31 & \begin{tabular}{|l|}
5.21 \\
$(0.90)$ \\
\end{tabular} & 5.31 & 6.06 & 4.27 & $\begin{array}{l}-2.20 \\
(0.16) \\
\end{array}$ & -2.35 & -2.20 & -2.04 & $\begin{array}{l}7.15 \\
(0.76) \\
\end{array}$ \\
\hline Def1* & \begin{tabular}{|l|}
1.38 \\
$(0.96)$ \\
\end{tabular} & 1.81 & 2.05 & 0.28 & \begin{tabular}{|l|}
1.54 \\
$(0.96)$ \\
\end{tabular} & 1.91 & 2.26 & 0.45 & $\begin{array}{l}0.16 \\
(0.05)\end{array}$ & 0.10 & 0.20 & 0.17 & $\begin{array}{l}1.25 \\
(0.85) \\
\end{array}$ \\
\hline Dsk2 & $\begin{array}{l}0.95 \\
(0.84) \\
\end{array}$ & 0.50 & 1.92 & 0.43 & \begin{tabular}{|l|}
1.13 \\
$(0.84)$ \\
\end{tabular} & 1.50 & 1.73 & 0.17 & $\begin{array}{l}0.18 \\
(0.71) \\
\end{array}$ & 1.00 & -0.19 & -0.26 & $\begin{array}{l}1.24 \\
(0.64) \\
\end{array}$ \\
\hline Kel1 ${ }^{* *}$ & \begin{tabular}{|l|}
0.95 \\
$(0.52)$ \\
\end{tabular} & 0.77 & 1.53 & 0.54 & \begin{tabular}{|l|}
0.85 \\
$(0.55)$ \\
\end{tabular} & 0.70 & 1.45 & 0.39 & $\begin{array}{l}-0.10 \\
(0.05) \\
\end{array}$ & -0.07 & -0.08 & -0.15 & \\
\hline Sis $1^{* *}$ & \begin{tabular}{|l|}
0.76 \\
$(0.33)$ \\
\end{tabular} & 1.02 & 0.86 & 0.39 & \begin{tabular}{|l|}
0.66 \\
$(0.26)$ \\
\end{tabular} & 0.91 & 0.66 & 0.39 & $\begin{array}{l}-0.10 \\
(0.10) \\
\end{array}$ & -0.10 & -0.20 & 0.00 & $\begin{array}{l}0.73 \\
(0.79) \\
\end{array}$ \\
\hline She2** & \begin{tabular}{|l|}
$\mathbf{0 . 6 6}$ \\
$(0.21)$ \\
\end{tabular} & - & 0.80 & 0.51 & \begin{tabular}{|l|}
0.57 \\
$(0.28$ \\
\end{tabular} & - & 0.77 & 0.38 & $\begin{array}{l}-0.04 \\
(0.09) \\
\end{array}$ & .05 & -0.03 & -0.13 & \\
\hline Spt5* & \begin{tabular}{|l|}
0.57 \\
$(0.33)$ \\
\end{tabular} & 0.80 & - & 0.34 & \begin{tabular}{|l|}
0.57 \\
$(0.33)$ \\
\end{tabular} & 0.53 & 0.91 & 0.25 & $\begin{array}{l}-0.17 \\
(0.13) \\
\end{array}$ & -0.26 & - & -0.08 & \\
\hline Prx1 & \begin{tabular}{|l|}
0.56 \\
$(0.01)$ \\
\end{tabular} & & 0.56 & 0.57 & \begin{tabular}{|l|}
0.16 \\
$(0.08)$ \\
\end{tabular} & & 0.11 & 21 & $\begin{array}{l}-0.44 \\
(0.07) \\
\end{array}$ & -0.50 & -0.45 & -0.36 & \\
\hline Ssd1 & $\begin{array}{l}\mathbf{0 . 5 4} \\
(0.32) \\
\end{array}$ & 0.40 & 0.91 & 0.31 & \begin{tabular}{|l|}
0.67 \\
$(0.42)$ \\
\end{tabular} & 0.62 & 1.12 & 0.28 & $\begin{array}{l}0.14 \\
(0.14) \\
\end{array}$ & 23 & 0.21 & -0.03 & \\
\hline $\mathrm{Mcm6}^{* *}$ & $\begin{array}{l}\mathbf{0 . 4 2} \\
(0.14)\end{array}$ & 0.56 & 0.42 & 0.28 & \begin{tabular}{|l|}
0.37 \\
$(0.16)$ \\
\end{tabular} & 0.54 & 0.33 & 0.23 & $\begin{array}{l}-0.05 \\
(0.04) \\
\end{array}$ & -0.01 & -0.09 & -0.06 & $\begin{array}{l}0.45 \\
(0.28) \\
\end{array}$ \\
\hline \multicolumn{14}{|c|}{ only 0-1 proteome-corrected SILAC-ratio, 2-3 enrichment ratios $\geq 0.26$} \\
\hline Mnl2* & - & - & - & 5.09 & $\begin{array}{l}\mathbf{5 . 5 0} \\
(0.83) \\
\end{array}$ & - & 6.33 & 67 & - & - & - & -0.42 & $\begin{array}{l}4.92 \\
(0.92) \\
\end{array}$ \\
\hline Nop1*3 & - & - & - & 0.50 & \begin{tabular}{|l|}
$\mathbf{0 . 6 3}$ \\
$(0.27)$ \\
\end{tabular} & 0.54 & 1.00 & 0.35 & - & - & - & -0.14 & \\
\hline Cue $5^{* *}$ & - & - & - & 0.36 & $\begin{array}{l}\mathbf{0 . 6 0} \\
(0.10) \\
\end{array}$ & 0.68 & 0.46 & 0.67 & - & - & - & 0.32 & $\begin{array}{l}0.65 \\
(0.10) \\
\end{array}$ \\
\hline $\operatorname{Rav1*}$ & - & - & - & - & \begin{tabular}{|l|}
$\mathbf{0 . 5 8}$ \\
$(0.23)$ \\
\end{tabular} & 0.60 & 0.30 & 0.85 & - & - & - & - & $\begin{array}{l}0.44 \\
(0.28) \\
\end{array}$ \\
\hline $\mathrm{Mcm}^{*}$ & - & - & - & 0.28 & \begin{tabular}{|l|}
0.51 \\
$(0.18)$ \\
\end{tabular} & 0.61 & 0.67 & 0.27 & - & - & - & -0.01 & $\begin{array}{l}0.16 \\
(0.08) \\
\end{array}$ \\
\hline Gsy1 & - & - & - & & $\begin{array}{l}0.50 \\
(0.19)\end{array}$ & 0.68 & 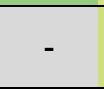 & 0.31 & - & - & - & - & $\begin{array}{l}0.25 \\
(0.27)\end{array}$ \\
\hline
\end{tabular}

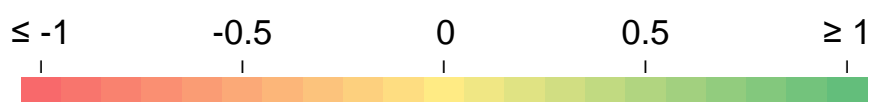


Table 6. Logarithmized SILAC-ratios of proteins co-captured with Asc1DEp-Strep.

The listed proteins were found to be enriched together with Strep-tagged Asc1DEp based on their relative quantification using SILAC. The layout of the table and abbreviations/symbols are as described for Table 5. (wt = wild-type, mean Asc1p-Strep/control SILAC-ratios equivalent to DE in Table 5).

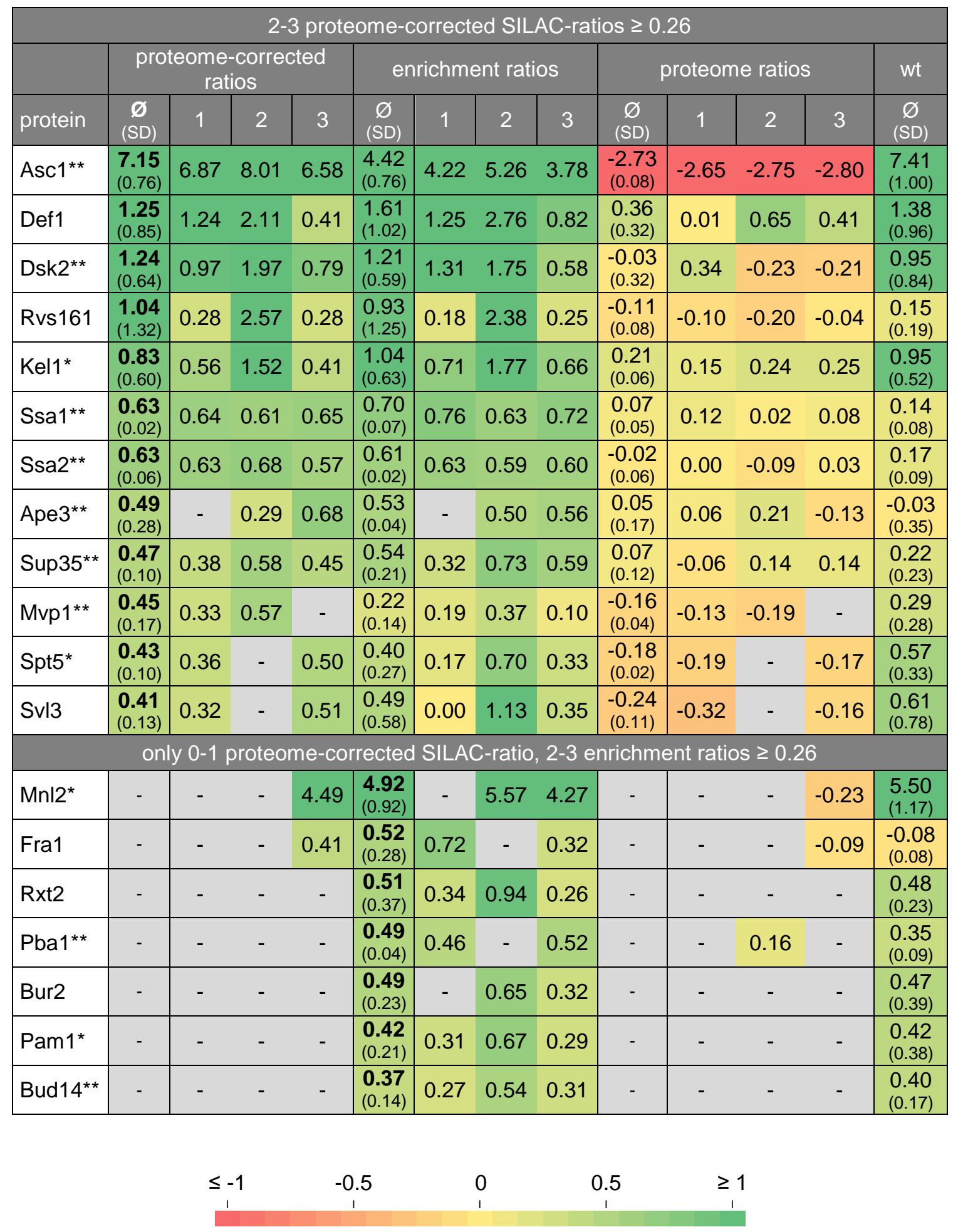


cultures directly after their cultivation, thereby treating them in the following steps as one sample and preventing any experimental bias due to individual sample processing. However, light and heavy labeled forms of proteins bound to the bait protein might interchange during the enrichment procedure if their interaction is highly dynamic (Wang and Huang, 2008). One possibility to address this problem is to pool samples at a later stage. Here, the cell lysis of independent cultures was done separately, and samples were pooled immediately prior to the affinity purification of Strep-tagged Asc1p (Figure 23). Strep-Tactin ${ }^{\circledR}$ spin columns were used that enable fast downstream processing of the samples after their pooling. Using a triple SILAC approach, three different strains expressing either Asc1p-Strep, Asc1DEp-Strep, or the untagged protein as a negative control were processed in a single affinity purification experiment. The proteins were expressed from high copy plasmids $(2 \mu \mathrm{m})$ under control of the MET25-promoter in the $\Delta a s c 1 \Delta a r g 4 \Delta l y s 1$ strain. In total, three biological replicates were analyzed. The eluate fraction and a sample from the total protein extract (after pooling but prior to affinity purification) were subjected to SDS-PAGE followed by in-gel trypsin digestion. Peptide samples were analyzed with LC-MS, and the obtained raw data files were analyzed against an S. cerevisiae-specific protein database using the MaxQuant software with the Andromeda search engine for the identification and quantification of peptides and their corresponding proteins. SILAC-ratios were reported with the values for the Asc1p control in the denominator so that high ratio values indicate the Asc1p-dependent co-enrichment of a protein. To avoid misinterpretation due to expression effects, the total protein extracts were analyzed as well to get quantitative proteome data as an input-control. SILAC-ratios for proteins of the enriched fractions were corrected for SILAC-ratios of proteins from the proteome (as it was described in chapter 3.3.1 for the correction of phospho-peptide ratios with protein ratios). Reduced sample complexity derived from the enrichment led to identifications (and quantifications) of proteins that were not covered from the very complex samples obtained from total protein extracts. Thus, the described proteome-correction of SILAC-ratios was not applicable to all proteins.

The MaxQuant data were downstream analyzed using the Perseus software as briefly illustrated in Figure 23. For a detailed overview of all processing steps, see Supplemental Table 13. The normalized SILAC-ratios from the MaxQuant protein groups output file were used in Perseus. The normalization step of MaxQuant centers the normal distribution of SILAC-ratios on a ratio of 1:1 for the bulk of proteins, thereby correcting experimental biases. This mathematical correction is performed under the assumption that most proteins do not differ in their abundance between the samples. 
Values were logarithmized $\left(\log _{2}\right)$ so that a ratio value of 0 indicates no enrichment, and a ratio value considerably greater than 0 hints to a specific enrichment of a protein through its association with Asc1p-Strep or Asc1DEp-Strep. Two-sample $t$-tests (p-value $<0.1$ and pvalue $<0.05)$ were applied to obtain statistical validation for the enrichment of candidates. For each protein, the SILAC-ratios from the enriched fractions (x) were proteome-corrected with the respective SILAC-ratios from the proteome (y) by subtraction (x-y). All proteomecorrected SILAC-ratios of the three replicates had to be $\geq 0.26$ (approximately 20\% enrichment) to identify a protein as a putative Asc1p-Strep interaction partner. In case that only two ratios were determined, both had to be $\geq 0.26$. These thresholds resulted in the identification of nine proteins for Asc1p-Strep and eleven proteins for Asc1DEp-Strep plus the bait proteins Asc1-Strep and Asc1DE-Strep.

For proteins with no or only one proteome-corrected SILAC-ratio, the same filter (two values $\geq 0.26$ and no value $<0.26$ ) was applied on the non-proteome-corrected SILAC-ratios from the eluate fraction. If a proteome-corrected SILAC-ratio was available, this should not be less than 0.26. With these thresholds six and seven proteins were additionally considered as enriched together with Asc1p-Strep and Asc1DEp-Strep, respectively. Additionally, a onesample $t$-test instead of a two-sample $t$-test was applied on these proteins for statistical validation of candidates.

All candidates and their quantification values are listed in Tables 5 and 6, and the resulting Asc1p-interaction map is depicted in Figure 24. Five proteins were found as putative interaction partners for Asc1p-Strep as well as for Asc1DEp-Strep namely Def1p, Dsk2p, Kel1p, Mnl2p, and Spt5p. The remaining 23 proteins were identified for the enrichment of only one of the two Asc1p variants. The interaction map also indicates interactions between proteins that were already reported in literature (according to interactions listed in SGD). Bud14p is the only protein that has been reported as a physical interaction partner of Asc1p before. Bud $14 p$ is one of the regulatory subunits of the phosphatase Glc7p and might therefore be directly responsible for Asc1p-mediated dephosphorylation processes (Knaus et al., 2005). As a regulatory subunit of the Bur1p protein kinase, the Asc1DEp-Strep-associated cyclin Bur2p is involved in regulation of phosphorylation as well (Yao et al., 2000). Additionally, three proteins with Asclp-sensitive phosphorylation sites namely Def1p, Sup35p, and Ssd1p were found. 

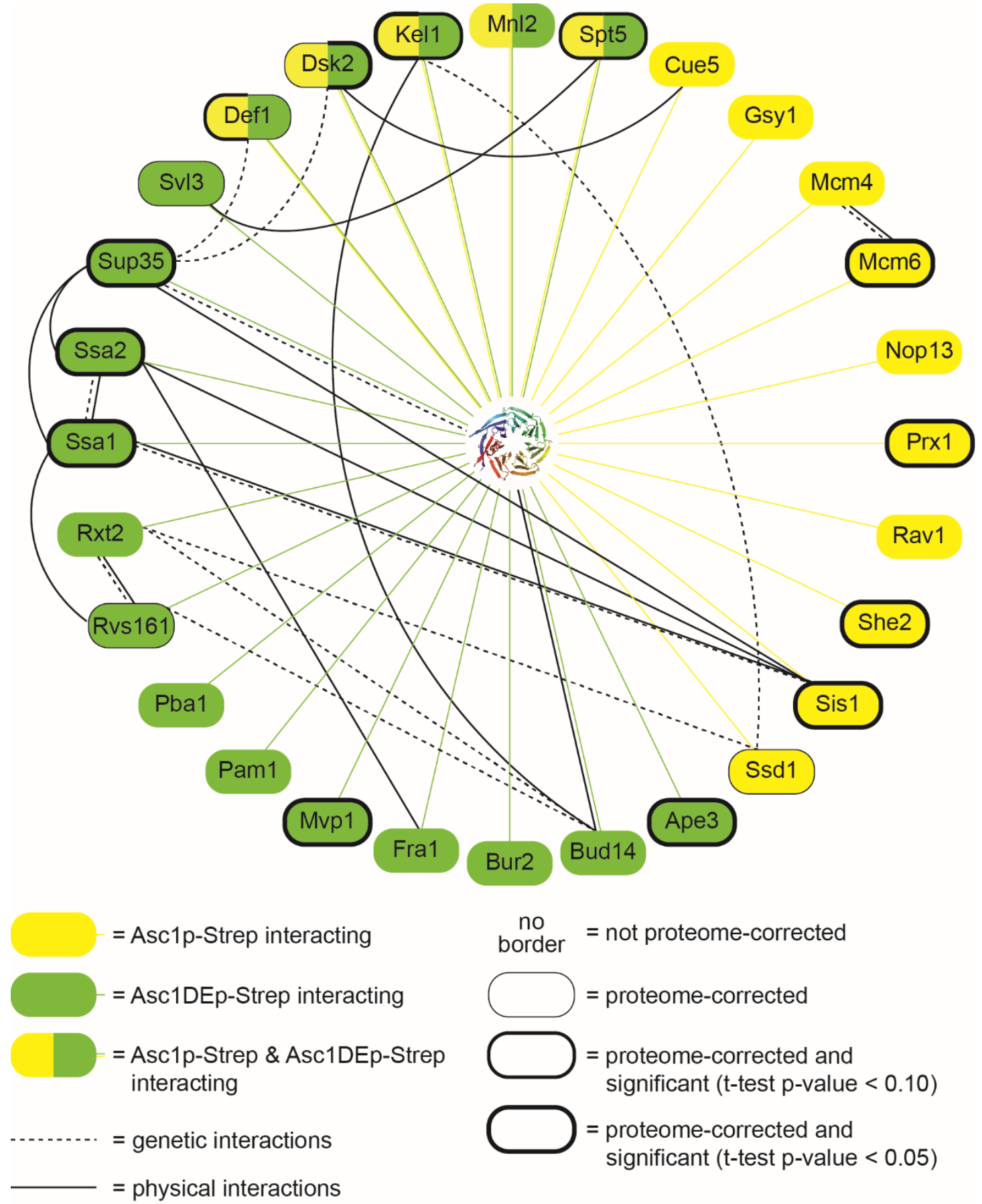

Figure 24. Interaction map of Asc1p-Strep and Asc1DEp-Strep.

All proteins identified as putative interaction partners of Asc1p-Strep (colored in yellow) or Asc1DEpStrep (colored in green). Proteins highlighted in both colors were found to interact with both Asc1p variants. The Asclp structure is placed in the center, and the colored lines illustrate the interactions identified in this work. (The structure figure of Asc1p was generated with the PyMOL Molecular Graphics System software on the basis of the PDB file 3FRX). Black lines and dashed black lines connect proteins that have already been reported as physical or genetic interaction partners, respectively, in other studies according to SGD. For proteins without a black border, no or only one proteome-corrected SILAC-ratio could be obtained. A black border indicates that at least two proteome-corrected SILAC-ratios could be obtained for the respective protein. Thick black borders indicate that the two-sample $t$-test was positive (p-value $<0.1$ or $\mathrm{p}$-value $<0.05$ ). 


\subsection{The Asc1p-dependent translatome}

Based on its exposed position at the head of the 40S ribosomal subunit next to the mRNA exit tunnel, Asc1p is considered to be directly involved in regulation of translation (Sengupta et al., 2004). It has been shown to interact with translation factors and mRNA-binding proteins and might affect translation in general or for specific transcripts (Baum et al., 2004; Kouba et al., 2012). Here, the translatomes - meaning the entity of actively translated mRNAs - of $A S C 1$ wild-type and the asc $1^{-}$cells were quantitatively compared to identify transcripts that are targets of an Asc1p-dependent translational regulation.

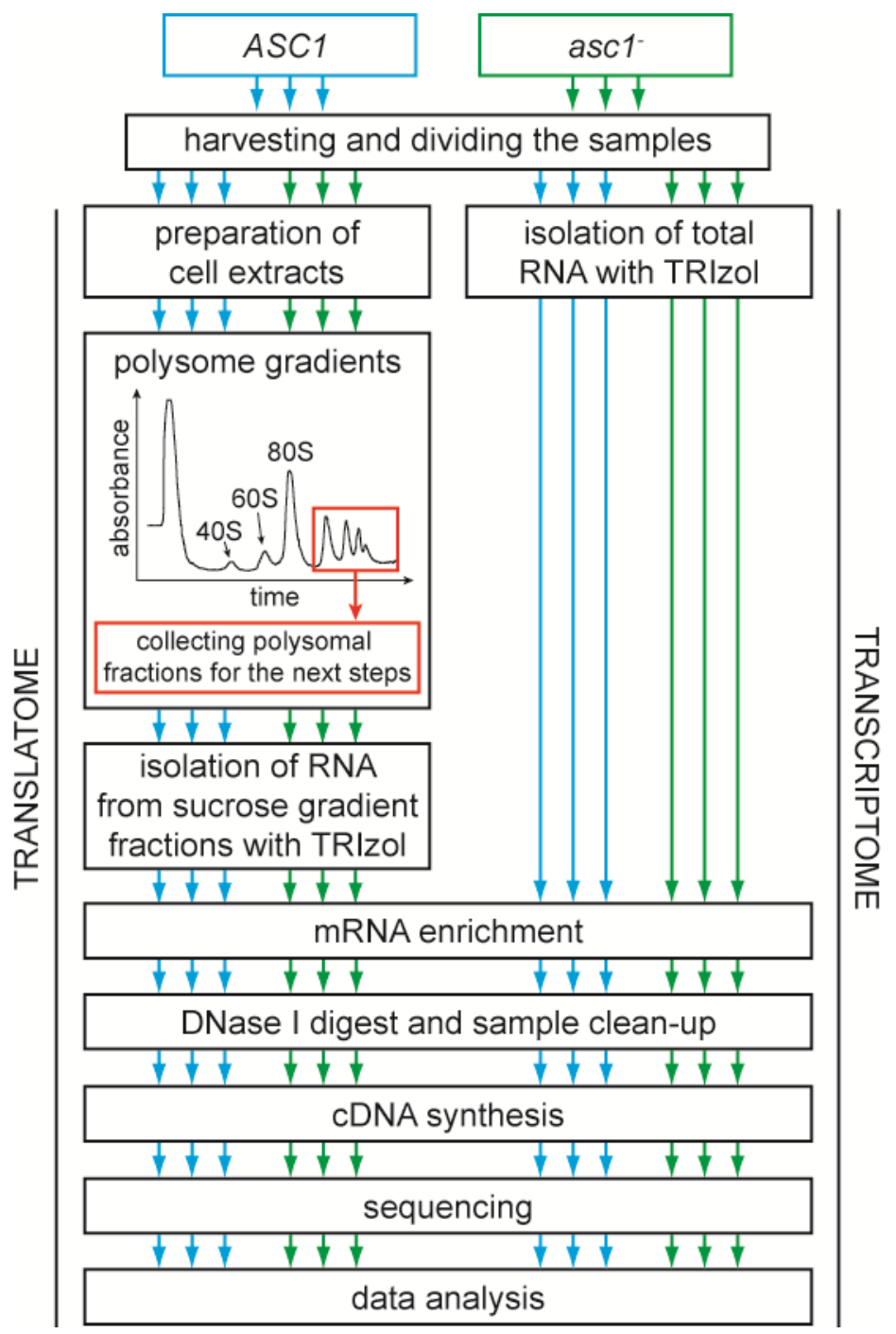

Figure 25. Workflow for the Asc1p-dependent translatome and transcriptome analysis.

The ASCl wild-type and asc $^{-}$strains were cultivated in three replicates. After harvesting of the cells, each sample was divided and one half processed for the translatome and the other half for the transcriptome analysis. For the translatome analysis, cell extracts were prepared and subjected to sucrose-density ultracentrifugation. Polysomal fractions were collected, and RNA was isolated. For the transcriptome analysis, total RNA was directly isolated from the cells. RNA samples from both experimental lines were subjected to mRNA enrichment and DNase I digest. cDNA was synthesized from the RNA, and samples were subjected to sequencing followed by data analysis. 
Table 7. Asc1p-dependent changes in the translatome.

An expanded view of this table with the normalized readcounts for all three replicates for each strain can be found in Supplementary Table 14. For each transcript and strain, the mean of the normalized readcounts was calculated. Logarithmized $\left(\log _{2}\right)$ ascl-/ASC1 ratios of these mean values are provided

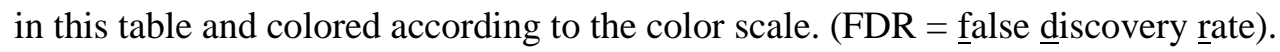

\begin{tabular}{|l|c|c|c|}
\hline \multicolumn{1}{|c|}{ gene } & $\begin{array}{c}\text { asc1-1 } \\
\text { ASC1 }\end{array}$ & $\begin{array}{c}\text { likeli- } \\
\text { hood }\end{array}$ & FDR \\
\hline COS8 & 2.68 & 0.9965 & $1.7 \mathrm{E}-03$ \\
\hline ZPS1 & 2.35 & 0.9973 & $1.3 \mathrm{E}-03$ \\
\hline PRM5 & 1.84 & 0.9981 & $7.7 \mathrm{E}-04$ \\
\hline HBN1 & 1.69 & 0.9995 & $1.4 \mathrm{E}-04$ \\
\hline GSY1 & 1.65 & 0.9994 & $2.2 \mathrm{E}-04$ \\
\hline HSP42 & 1.61 & 0.9916 & $3.5 \mathrm{E}-03$ \\
\hline HSP26 & 1.48 & 0.9160 & $3.2 \mathrm{E}-02$ \\
\hline YCL021W-A & 1.45 & 0.9909 & $4.0 \mathrm{E}-03$ \\
\hline GAL3 & 1.38 & 0.9595 & $1.3 \mathrm{E}-02$ \\
\hline YLL053C & 1.36 & 0.9834 & $5.5 \mathrm{E}-03$ \\
\hline ZRT1 & 1.35 & 0.9397 & $1.8 \mathrm{E}-02$ \\
\hline YKL070W & 1.34 & 0.9268 & $2.8 \mathrm{E}-02$ \\
\hline FET4 & 1.32 & 0.9935 & $2.6 \mathrm{E}-03$ \\
\hline VTI1 & 1.27 & 0.9608 & $1.2 \mathrm{E}-02$ \\
\hline AHA1 & 1.26 & 0.9973 & $1.1 \mathrm{E}-03$ \\
\hline AQY2 & 1.22 & 0.9322 & $2.4 \mathrm{E}-02$ \\
\hline FKS3 & 1.22 & 0.9886 & $4.5 \mathrm{E}-03$ \\
\hline STI1 & 1.17 & 0.9983 & $6.0 \mathrm{E}-04$ \\
\hline AGA1 & 1.16 & 0.9495 & $1.5 \mathrm{E}-02$ \\
\hline GAL80 & 1.15 & 0.9826 & $5.9 \mathrm{E}-03$ \\
\hline GSY2 & 1.12 & 0.9986 & $4.2 \mathrm{E}-04$ \\
\hline NDJ1 & 1.07 & 0.9298 & $2.5 \mathrm{E}-02$ \\
\hline URA10 & 1.05 & 0.9182 & $3.1 \mathrm{E}-02$ \\
\hline HXK1 & 1.05 & 0.9409 & $1.7 \mathrm{E}-02$ \\
\hline GRX3 & 1.02 & 0.9943 & $2.2 \mathrm{E}-03$ \\
\hline SMA2 & 1.01 & 0.9343 & $2.2 \mathrm{E}-02$ \\
\hline DCS1 & 1.01 & 0.9921 & $3.3 \mathrm{E}-03$ \\
\hline YGR161W-C & 1.00 & 0.9970 & $1.5 \mathrm{E}-03$ \\
\hline YHB1 & 0.95 & 0.9749 & $8.0 \mathrm{E}-03$ \\
\hline UBC1 & 0.91 & 0.9280 & $2.6 \mathrm{E}-02$ \\
\hline LST8 & 0.91 & 0.9278 & $2.7 \mathrm{E}-02$ \\
\hline ALG13 & 0.90 & 0.9891 & $4.2 \mathrm{E}-03$ \\
\hline HSP78 & 0.86 & 0.9564 & $1.4 \mathrm{E}-02$ \\
\hline APE1 & 0.84 & 0.9644 & $1.2 \mathrm{E}-02$ \\
\hline YMR315W & 0.84 & 0.9340 & $2.3 \mathrm{E}-02$ \\
\hline FMP41 & 0.82 & 0.9207 & $2.8 \mathrm{E}-02$ \\
\hline GLO2 & 0.80 & 0.9352 & $2.2 \mathrm{E}-02$ \\
\hline
\end{tabular}

\begin{tabular}{|l|c|c|c|}
\hline \multicolumn{1}{|c|}{ gene } & $\begin{array}{c}\text { asc1/ } \\
\text { ASC1 }\end{array}$ & $\begin{array}{c}\text { likeli- } \\
\text { hood }\end{array}$ & FDR \\
\hline GLK1 & 0.78 & 0.9392 & $1.9 \mathrm{E}-02$ \\
\hline TWF1 & 0.77 & 0.9140 & $3.2 \mathrm{E}-02$ \\
\hline MXR1 & 0.76 & 0.9046 & $3.4 \mathrm{E}-02$ \\
\hline SFG1 & 0.76 & 0.9011 & $3.5 \mathrm{E}-02$ \\
\hline ARC18 & 0.76 & 0.9075 & $3.3 \mathrm{E}-02$ \\
\hline YKL151C & 0.76 & 0.9710 & $8.6 \mathrm{E}-03$ \\
\hline EXG2 & 0.71 & 0.9366 & $2.0 \mathrm{E}-02$ \\
\hline YDR327W & 0.70 & 0.9187 & $3.0 \mathrm{E}-02$ \\
\hline DFR1 & -0.66 & 0.9357 & $2.1 \mathrm{E}-02$ \\
\hline HTB1 & -0.68 & 0.9396 & $1.8 \mathrm{E}-02$ \\
\hline FAA2 & -0.75 & 0.9290 & $2.5 \mathrm{E}-02$ \\
\hline HO & -0.75 & 0.9699 & $1.0 \mathrm{E}-02$ \\
\hline TDA1 & -0.91 & 0.9708 & $9.1 \mathrm{E}-03$ \\
\hline YHR177W & -0.93 & 0.9188 & $2.9 \mathrm{E}-02$ \\
\hline URA4 & -1.02 & 0.9922 & $3.1 \mathrm{E}-03$ \\
\hline AAC3 & -1.02 & 0.9702 & $9.7 \mathrm{E}-03$ \\
\hline SNO1 & -1.04 & 0.9755 & $7.5 \mathrm{E}-03$ \\
\hline ARN1 & -1.06 & 0.9594 & $1.4 \mathrm{E}-02$ \\
\hline ALD6 & -1.06 & 0.9682 & $1.1 \mathrm{E}-02$ \\
\hline YJL213W & -1.16 & 0.9800 & $7.0 \mathrm{E}-03$ \\
\hline BAP3 & -1.23 & 0.9802 & $6.7 \mathrm{E}-03$ \\
\hline YIL165C & -1.25 & 0.9970 & $1.4 \mathrm{E}-03$ \\
\hline AQR1 & -1.29 & 0.9823 & $6.2 \mathrm{E}-03$ \\
\hline ECM13 & -1.36 & 0.9960 & $2.0 \mathrm{E}-03$ \\
\hline SNZ1 & -1.47 & 0.9933 & $2.8 \mathrm{E}-03$ \\
\hline RIB4 & -1.49 & 0.9976 & $9.5 \mathrm{E}-04$ \\
\hline TPO1 & -1.64 & 0.9961 & $1.8 \mathrm{E}-03$ \\
\hline YGR035C & -1.64 & 0.9909 & $3.7 \mathrm{E}-03$ \\
\hline YMR141W-A & -1.67 & 0.9491 & $1.6 \mathrm{E}-02$ \\
\hline FMP48 & -1.71 & 0.9877 & $4.8 \mathrm{E}-03$ \\
\hline TPO4 & -1.74 & 0.9650 & $1.1 \mathrm{E}-02$ \\
\hline TIS11 & -1.86 & 0.9857 & $5.1 \mathrm{E}-03$ \\
\hline URA3 & -1.87 & 0.9936 & $2.4 \mathrm{E}-03$ \\
\hline URA1 & -2.14 & 1.0000 & $2.5 \mathrm{E}-05$ \\
\hline EEB1 & -2.56 & 1.0000 & $8.6 \mathrm{E}-06$ \\
\hline MDH2 & 1.0000 & $2.0 \mathrm{E}-05$ \\
\hline
\end{tabular}

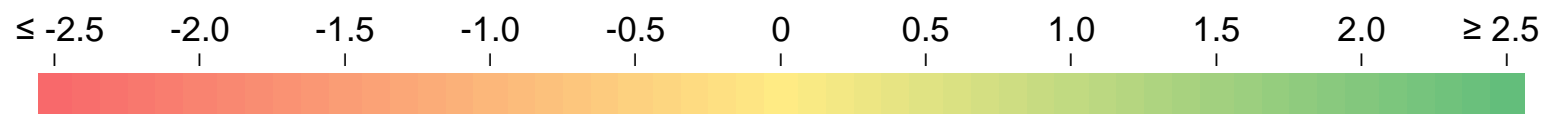


The experiments were performed with three biological replicates for each strain (Figure 25). Total cell extracts derived from exponentially growing yeast cells were subjected to sucrose density ultracentrifugation to separate polysomes from ribosomal subunits, monosomes, and mRNP particles. The polysome fractions with highly translated mRNAs were collected, and RNA was isolated. The samples were enriched for mRNAs to reduce the rRNA amount in the samples. DNA in the samples had to be removed by enzymatic DNase I digestion. The RNA was transcribed into cDNA and subjected to sequencing. The readcounts for each mRNA were used for a differential expression analysis with the baySeq algorithm (Hardcastle and Kelly, 2010).

Transcripts differentially abundant in the translatomes of the ASCl wild-type strain and the ascl- $1^{-}$strain that had $\geq 90 \%$ likelihood for regulation were further considered. For the 73 transcripts that passed this threshold, the normalized readcounts of all six samples are given in the Supplementary Table 14. The mean value of the normalized readcounts was calculated for the three replicates of each strain, and the asc1\%/ASCl ratio of these values was determined (Table 7 and Supplementary Table 14). 45 polysomal transcripts were found to be increased and 28 decreased in their abundance in the $\mathrm{sscl}^{-}$strain in comparison to the ASCl wild-type strain.

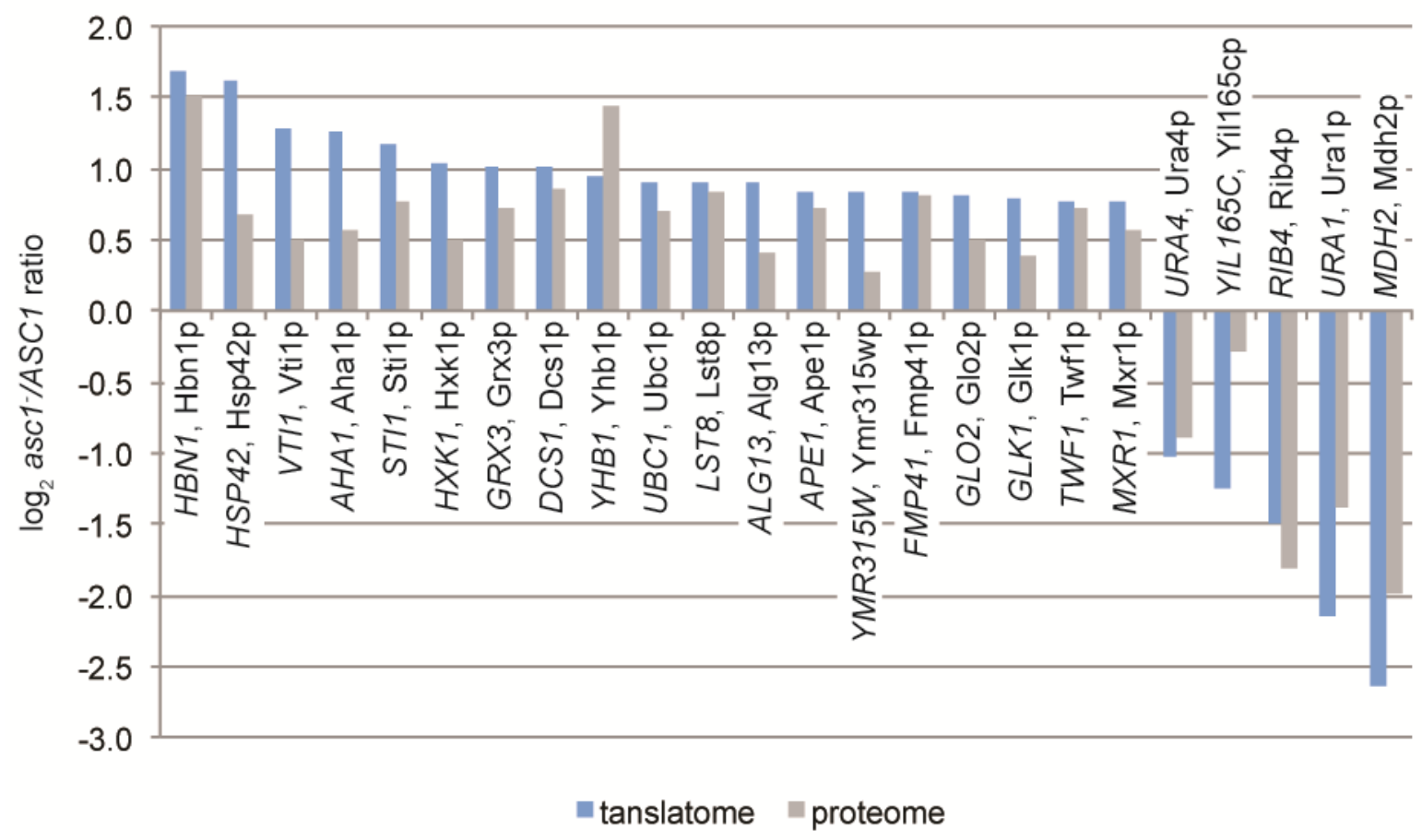

Figure 26. Asc1p-dependent translatome versus proteome.

The graph provides the $\log _{2}$ asc1-/ASC1 ratios for mRNAs and proteins that were found to be Asc1pdependently affected in their abundance in the translatome as well as in the total proteome. 
For 24 of the polysomal transcripts, a relative quantification value for the corresponding protein can be found in the list of Asc1p-dependently regulated proteins (Figure 26 and Supplementary Table 4). For all 24 candidates, the direction of regulation was identical in the translatome and proteome hinting to a correlation between the translation efficiency for an mRNA and the expression level of the respective mature protein. Alteration of the abundance of a transcript in the translatome could reflect the general changes in the abundance of the transcript. Therefore, the translatome data were compared with a former microarray-based quantitative transcriptome analysis for the $\Delta a s c 1$ strain (Rachfall et al., 2013). 13 transcripts were found to be altered in their abundance in the translatome as well as in the transcriptome (Figure 27). 12 of these transcripts showed the same direction of regulation in the translatome and the transcriptome. Thus, Asc1p-dependently increased or decreased abundance of these transcripts might lead to differences in their translation rates. Yet, one mRNA, the transcript of the $Y H R 177 \mathrm{~W}$ gene, was increased in its total abundance, but showed severely decreased association with translating ribosomes upon Asc1p-deficiency. YHR177W is a paralog of the gene encoding Muc1p expressed independent of $\underline{T E C 1} \underline{1}$ (Mit1p). Their gene products belong to the WOPR family of transcriptional regulators that is named for its members Worlp, Pac1p, and Ryp1 and contain a WOPR box for DNA-binding (Lohse et al., 2010). The WOPR box is highly conserved within the fungal kingdom and found in regulators of morphology and pathogenesis (Lohse et al., 2010). For example, Mit1p is required for pseudohypal growth in S. cerevisiae, and Worlp regulates the transition of yeast cells from white to opaque cell morphology in C. albicans (Huang et al., 2006; Cain et al., 2012).

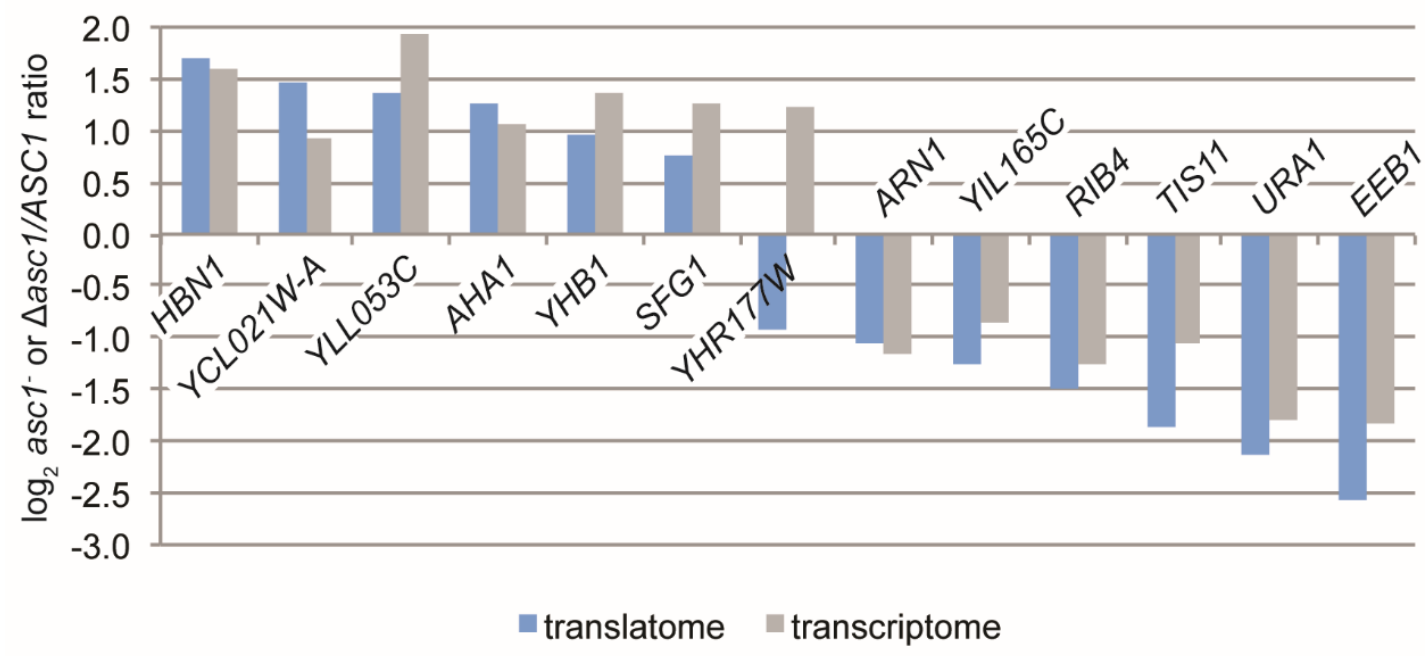

Figure 27. Asc1p-dependent translatome and transcriptome.

The columns provide the $\log _{2}$ asc1 1 ASC1 ratios for mRNAs found to be Asc1p-dependently affected in their levels in the translatome as well as in the transcriptome. Transcriptome data derived from a microarray analysis comparing the $A S C l$ wild-type strain with the $\triangle a s c 1$ strain (Rachfall et al., 2013). 
The comparison with the microarray data implicates that RNASeq-based transcriptome data for the $a s c 1^{-}$strain are required to differentiate between mRNAs that change in their abundance in the translatome due to an Asc1p-dependent regulation in their expression level or an Asc1p-dependent alteration of their translation efficiency. Experiments for a quantitative transcriptome analysis of the $a s c 1^{-}$strain with RNASeq are in progress (Figure 25). 


\section{Discussion}

\subsection{Does the R38D K40E amino acid exchange within Asc1p cause a ribosome binding defect in vivo?}

The Asc1 protein of $S$. cerevisiae is not essential for viability of yeast cells under laboratory conditions, but is required to adapt cells to a variety of different stress conditions. As a constituent of the ribosome, it is likely that Asc1p's molecular function is tightly linked to the process of mRNA translation. The crystal structures of the $T$. thermophila $40 \mathrm{~S}$ subunit and the S. cerevisiae 80 S ribosome confirm Asc1p's integral position within the ribosome (Ben-Shem et al., 2011; Rabl et al., 2011). In cells of the stationary growth phase of a yeast culture, Asc1p seems to bind ribosomes less tightly (Baum et al., 2004). This gives rise to the question whether Asc1p also acts apart from the ribosome as it has been suggested for other ribosomal proteins (reviewed in Warner and McIntosh, 2009). Asc1p was described to function as the G-protein $\beta$-subunit of the $\mathrm{G} \alpha \mathrm{Gpa} 2 \mathrm{p}$, which is localized to membranes via its interaction with the nutrient sensor Gpr1p. A simultaneous interaction of Asc1p with Gpa2p and the ribosome was considered to be unlikely due to sterical hindrance (Zeller et al., 2007; Coyle et al., 2009). In metazoans, several known interactions of RACK1 also seem to occur in its ribosome-free state since the respective interaction surface was mapped to the ribosome-facing side of the $\beta$-propeller. Yet, homodimerization of Asc1p/RACK1 would enable a simultaneous interaction with the ribosome and a second interaction partner that binds to the same regions on the second $\beta$-propeller surface. Still, ribosome binding of RACK1 seems incompatible with the simultaneous interaction with the Fyn kinase and NR2B. The interaction with these two proteins in complex was proposed to rely on homodimer formation since the binding sites of both proteins involve amino acids 35 to 48 of RACK1 (Thornton et al., 2004). Many studies with metazoan systems do not even consider ribosome-localization of RACK1 for their experiments or the discussion of their results. In conclusion, a ribosome-independent impact of the $\beta$-propeller protein on cellular adaptation seems possible.

A ribosome binding mutated variant of Asc1p has been proposed and expected to contribute to the differentiation between ribosome-dependent and ribosome-independent functions of Asc1p. The mutated Asc1 protein contains an exchange of the two highly conserved residues R38 and K40 to aspartate (D) and glutamate (E), respectively, which weakens the binding to the ribosome (Sengupta et al., 2004; Coyle et al., 2009). In comparison to wild-type Asc1p, Asc1DEp is shifted from the ribosomal to the ribosome-free fraction during sucrose-gradient ultracentrifugation (Coyle et al., 2009). However, S. cerevisiae asclDE strains behave phenotypically mainly like the wild-type ASCl strain (this study; Coyle et al., 2009). The 
rather marginal phenotypic effects are confirmed here and reflected by our findings on the molecular level: While in Asc1p-deficient cells the protein levels of the transcription factors Flo8p, Tec1p, and Rap1p are significantly reduced and more than 600 additional proteins are altered in their abundance, the transcription factors are not affected by the DE exchange, and there are almost no changes observed within the total proteome. Yet, Kuroha and colleagues (2010) were able to show that a promoting effect of Asc1p on nascent polypeptide-dependent translation arrest is lost in the asclDE mutant strain.

It is surprising that a reduction in ribosome-association of almost 90\% (Coyle et al., 2009) should have only a minor impact on the functionality of Asc1p in S. cerevisiae. This would suggest either that Asc1p acts to a great extent independently from the ribosome or that a small percentage of Asc1p bound to ribosomes is sufficient for the protein to function. However, it has to be considered that the reduced ribosome-association of Asc1DEp in sucrose gradient ultracentrifugation might not reflect the in vivo situation: Although the DE mutation obviously compromises the interaction of Asc1p with the ribosome, its dissociation from the 40S subunit might only occur during cell lysis or subsequent ultracentrifugation.

It also has to be taken into consideration that the DE mutation impairs the interaction of Asc1p with other components besides the ribosome. For example, the interaction of mammalian RACK1 with the Fyn kinase as well as with the NR2B-subunit of the NMDA receptor involves amino acids 35 to 48 of RACK1 (Thornton et al., 2004), and R36 and K38 of mammalian RACK1 correspond to R38 and K40 of Asc1p. In S. pombe, exchange of these residues (R36 and K38) to alanine in combination with simultaneous R125A and K127A substitutions reduced the association of $\mathrm{Cpc} 2 \mathrm{p}$ with Ran1p, a protein kinase regulating the transition between mitosis and meiosis (McLeod et al., 2000). This study was published four years before the R38D K40E exchange was first shown to affect ribosome binding of Asc1p (Sengupta et al., 2004). These two residue pairs were chosen for mutagenesis because they are both part of a short motif that is found in the sequence of other direct interaction partners of Ran1p and might be responsible for substrate specificity (McLeod et al., 2000).

Here, Asc1p-Strep as well as Asc1DEp-Strep were purified to identify potential interaction partners of the wild-type protein and the variant. The experimental set-up did not result in the enrichment of ribosomal proteins with the Strep-tagged wild-type Asc1p. Thus, no difference in ribosome-association was observed between Strep-tagged Asc1p and Asc1DEp. Yet, proteins were identified that were specifically enriched with one of the two Asc1p variants. For example, the mRNA-binding protein She2 was exclusively enriched with Asc1p-Strep, whereas the heat shock proteins Ssa1 and Ssa2 and the translation termination factor Sup35p 
were only co-purified with Asc1DEp-Strep (see below for the discussion of these candidates and their relation to Asc1p). The direct or indirect interaction of these proteins with Asc1p might involve residues R38 and K40 as it was described for the Cpc2p-Ran1p interaction.

DE variants have also been studied in other organisms to analyze the effect of a reduced ribosome binding on the function of $\mathrm{Cpc} 2 \mathrm{p} / \mathrm{RACK} 1$. In $S$. pombe, a cpc2DE strain shows neither increased sensitivity to 3-AT nor decreased phosphorylation of eIF2 $\alpha$ as it was observed for the $\Delta c p c 2$ strain (Tarumoto et al., 2013). Yet, another study reported that expression of plasmid-borne wild-type Cpc2p, but not Cpc2DEp, rescues the reduced protein abundance of the MAPK phosphatases Pyp1p and Pyp2p and of the stress-responsive transcription factor Atf1p in a $\Delta c p c 2$ strain (Nuñez et al., 2009). Furthermore, expression of Cpc2DEp results in the same defect in $\mathrm{G}_{2} / \mathrm{M}$ transition as caused by a CPC2 deletion evident through increased cell size at cell division (Nuñez et al., 2009; Nuñez et al., 2010).

The following paragraph provides four examples for observations made with the RACK1-DE variant in metazoan systems. Arimoto and colleagues (2008) observed translocation of RACK1 to stress granules upon certain stress conditions presumably as part of the small ribosomal subunit. RACK1-DE did not show this cellular translocation implicating a reduction in ribosome-association of this RACK1 variant in vivo (Arimoto et al., 2008). In D. melanogaster S2 cell lines, a stable knock-down of RACK1 decreases propagation of viruses of the Dicistroviridae family due to the requirement of RACK1 for IRES-mediated translation of the viral mRNA. Transfection of this cell line with a vector carrying the RACK1-DE variant cannot rescue this phenotype in contrast to transfection with the same vector carrying the wild-type RACK1 gene (Majzoub et al., 2014). The RACK1-DE mutant was further used to ascribe an enhancing effect of RACK1 on chemoresistance and growth of human hepatocellular carcinoma to the ribosome-bound state of the protein (Ruan et al., 2012). The authors of this study showed, for example, that the transfection of Huh7 cells with wild-type RACK1, but not with RACK1-DE, mediates resistance against doxorubicin-induced apoptosis. Whereas overexpression of RACK1 promotes de novo protein biosynthesis in metabolic labeling studies in Huh7 cells, overexpression of RACK1-DE slightly inhibits this process. This observation might be caused by the reduced binding of the RACK1 interaction partner PKC $\beta I$ to the ribosome in the presence of RACK1-DE and consequently decreased phosphorylation of the PKC 3 II-targets eIF4E and eIF6 (Ruan et al., 2012). Gandin and colleagues (2013a) used the RACK1-DE variant to show that RACK1 recruits activated JNK to ribosomes. At the ribosome, JNK phosphorylates eEF1A2 that subsequently binds to 
newly synthesized polypeptides and induces their proteasomal degradation (Gandin et al., 2013a).

These results from the metazoan systems support the assumption that the DE exchange also causes in vivo reduction of ribosome-association of Asc1p/RACK1. Yet, it has to be considered that there might be substantial differences in the nature of Asc1p/RACK1's association with the ribosome between various organisms. The Leishmania major orthologue of Asc1p/RACK1, for example, harbors a glycin residue instead of the highly conserved lysine residue at position 36 and is nevertheless found tightly associated to ribosomes in sucrose-density centrifugation experiments (Choudhury et al., 2011). Furthermore, Coyle and colleagues (2009) also analyzed the importance of other lysine and arginine residues besides R38 and K40 at the ribosome-facing side of Asc1p for ribosome binding. The exchange of two less conserved residues (K62 and K87) to alanine affects the ribosome-association of Asc1p as well, yet to a significantly lesser extent than the R38D K40E mutation (Coyle et al., 2009; Adams et al., 2011).

Besides differences in characteristics of the interaction between Asc1p/RACK1 and the ribosome, there might be additional organism-specific factors, such as post-translational modification of Asc1p/RACK1, that influence its localization to the ribosome or at least its precise positioning at this site in vivo. Although the DE mutation had only marginal effects on Asc1p-dependent phenotypes, this work revealed tremendous synthetic effects when the DE mutation was combined with amino acid substitutions at Asc1p phospho-sites.

\subsection{The DE exchange and phospho-site mutations synergistically compromise Asc1p's functionality and integrity}

In this study, at least three previously unknown phosphorylation sites of Asc1p were identified: T12, T143, and Y250. These three residues and five additional phospho-sites of Asc1p (T96, T99, S120, S166, T168), which were known from previous high-throughput studies, were characterized in detail through the generation of respective ascl mutant strains (Chi et al., 2007; Smolka et al., 2007; Holt et al., 2009). The resulting strains synthesize Asc1p variants with replacements of the phospho-sites by either alanine/phenylalanine (dephosphorylation mimic) or by glutamate (phosphorylation mimic). The systematic phenotypic analysis of these strains revealed an increased sensitivity to translation inhibitors for the $a s c 1^{\mathrm{T} 143 \mathrm{~A}}$ and $a s c 1^{\mathrm{Y} 250 \mathrm{~F}}$ strains. All other phospho-site mutant strains showed wildtype behavior for the tested phenotypes. Yet, the combination of phospho-site mutations at T143 and Y250 as well as T12, T96, and T99 with the DE mutation caused strong synthetic 
phenotypes revealing the importance of these phospho-sites in combination with the conserved residues R38 and K40. Phosphorylation of Asc1p/RACK1 has also been reported in other organisms, such as S. pombe, H. sapiens, and A. thaliana (Chang et al., 2001; Liu et al., 2007b; Wilson-Grady et al., 2008; Kiely et al., 2009; Urano et al., 2015). The amino acids T12, T143, and Y250 of Asc1p are highly conserved in these organisms. (Figure 28, RACK1A is shown representatively for the three RACK1 proteins of A. thaliana). S. pombe

S.c. Asc1p
S.p. Cpc2p
H.s. RACK1
A.t. RACK1A

S.C. Asc1p
S.p. Cpc2p
H.s. RACK1
A.t. RACK1A
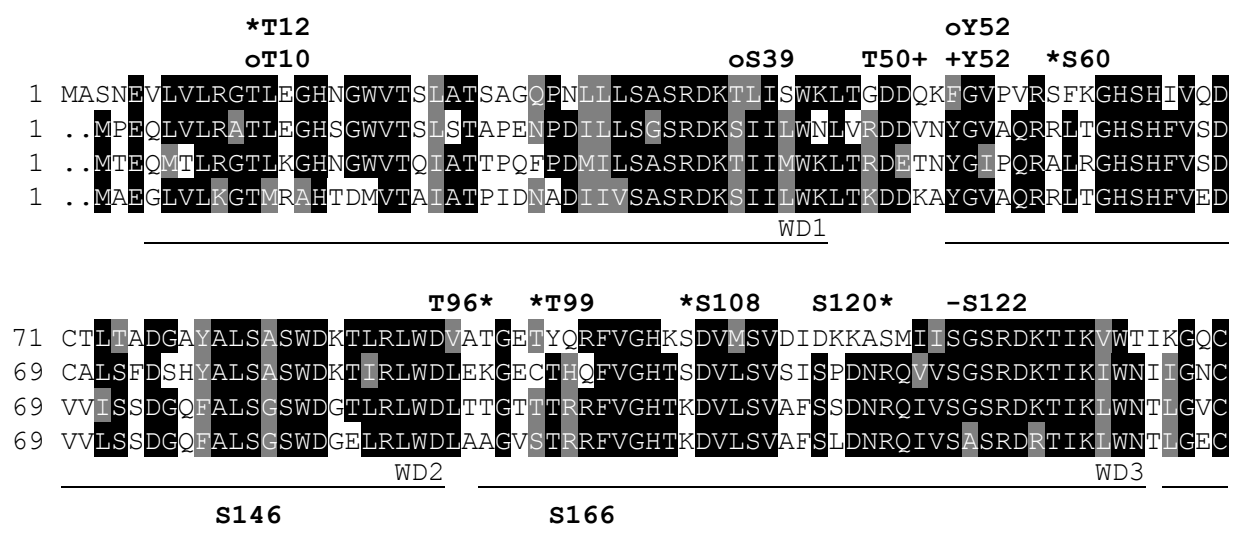

S.c. Asc1p

S.p. Сpc2p

H.s. RACK1

A.t. RACK1A
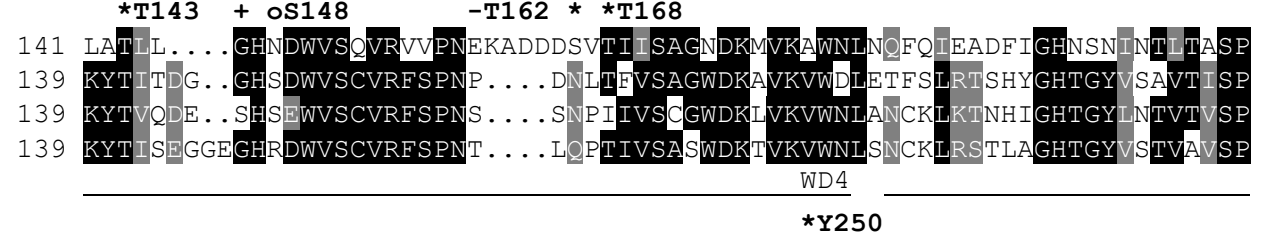

S.c. Asc1p

S.p. Cpc2p

H.s. RACK1

A.t. RACK1A

S.c. Asc1p

S.p. Cpc2p

H.s. RACK1

A.t. RACK1A
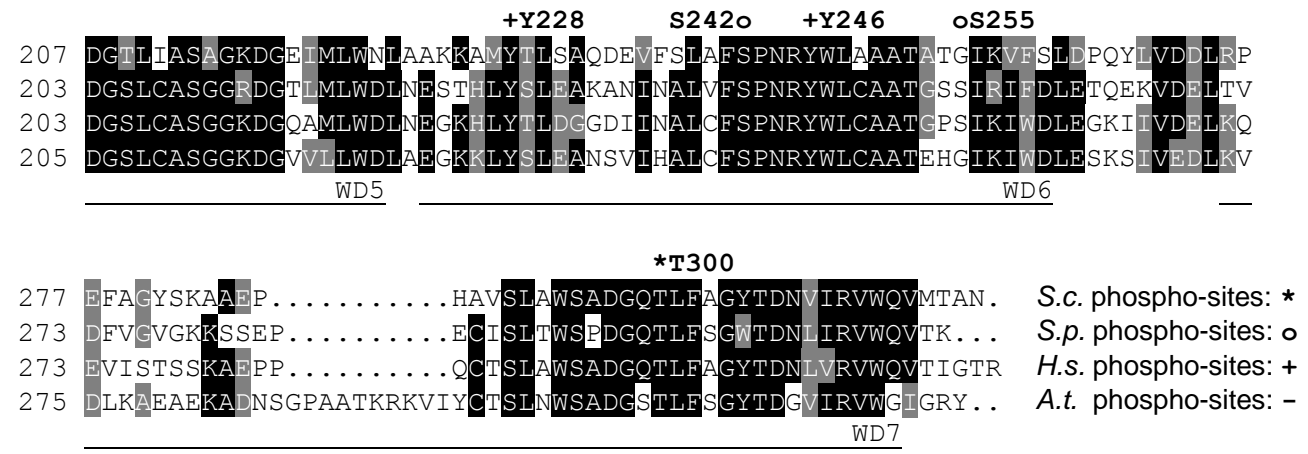

Figure 28. Phospho-site locations within Asc1p and its orthologues.

Amino acid sequence alignment of Asc1p with its orthologues and their phospho-sites. Amino acid residues that are conserved in at least three of the depicted sequences are shaded in black, those that are similar in at least three sequences are shaded in gray. Phospho-sites are indicated above the sequences according to the legend in the figure. For S. cerevisiae (S.c.) Asc1p, phospho-sites T12 (this study), S60 (Gnad et al., 2009), T96 and T99 (Chi et al., 2007), S108 and S120 (Holt et al., 2009), T143 (this study), S166 and T168 (Smolka et al., 2007), Y250 and T300 (this study) are indicated with an asterisk (*). For S. pombe (S.p.) Cpc2p, phospho-sites T10, S39, Y52, S148, S242, and S255 (Wilson-Grady et al., 2008) are labeled with a circle (o). Phospho-sites S122 and T162 of A. thaliana (A.t.) RACK1A (T161 of RACK1B and RACK1C, Urano et al., 2015) are indicated with a minus (-) and phospho-sites T50 (Zhao et al., 2015), Y52 (Kiely et al., 2009), S146 (Liu et al., 2007b), Y228 (Chang et al., 2001), and Y246 (Chang et al., 2001; Chang et al., 2002) of H. sapiens (H.s.) RACK1 are indicated with a plus (+). The seven WD40 repeats are labeled below the sequences. Sequences were retrieved from the UniProt database, aligned with the Clustal Omega program (http://www.uniprot.org/align) and shaded with the BoxShade tool (http://www.ch.embnet.org/ software/BOX_form.html). 
Cpc2p and human RACK1 have been reported to be phosphorylated at residues T10 and Y246, respectively, corresponding to positions T12 and Y250 of yeast Asc1p (Chang et al., 2001; Chang et al., 2002; Wilson-Grady et al., 2008). Phosphorylation of S. pombe Cpc2p was observed in a high-throughput study and has so far not been studied in detail. Here, impaired adhesive growth of the $\operatorname{ascl}^{\mathrm{T} 12 \mathrm{~A}} \mathrm{DE}$ strain was observed. Phosphorylation of human RACK1 at Y246 is catalyzed by the non-receptor protein tyrosine kinase Src. The modification enhances the interaction between RACK1 and Src leading to an inhibition of the kinase activity (Chang et al., 2001; Chang et al., 2002). Phosphorylation of RACK1 at Y246 also regulates its interaction with the mRNA-binding protein ZBP1, another target for the Src at the ribosome (Ceci et al., 2012). In our analyses, the ascl ${ }^{\mathrm{Y} 250 \mathrm{~F}}$ strain specifically showed sensitivity to inhibitors of mRNA translation suggesting a role of this phosphorylation site in (co-)translational processes. The mutation of mammalian RACK1 at Y246 does not reduce the association of the $\beta$-propeller protein to ribosomes (Ceci et al., 2012) suggesting that the observed phenotypes for the $a s c 1^{\mathrm{Y} 250 \mathrm{~F}}$ strain are not caused by enhanced release of the protein from the site of translation. However, impairment of Asc1p's correct localization to the ribosome through the DE mutation enhanced the effect of Y250F on the sensitivity of yeast cells to cycloheximide. To the best of our knowledge, this is the first study that showed the phosphorylation of Y250/Y246 of Asc1p/RACK1 in vivo. The high conservation of this amino acid residue and its surrounding sequence together with the experimental evidence for phosphorylation of Y246 in mammals suggest that the phosphorylation of this residue is highly conserved among eukaryotes and provides a common mechanism to regulate the protein.

According to the data compiled in the PhosphoGRID database only approximately $3 \%$ of all known phosphorylation sites in S. cerevisiae are tyrosine residues, whereas about $25 \%$ and $75 \%$ are threonine and serine residues, respectively (Stark et al., 2006). No S. cerevisiae homologues are known for the Src kinase or other members of the family of non-receptor protein tyrosine kinases. Furthermore, no true tyrosine kinases were described for yeast, yet, dual-specificity kinases that phosphorylate serine/threonine as well as tyrosine residues and function, for example, in MAPK cascades (Hunter and Plowman, 1997).

So far, amino acid T143 of Asc1p has not been described as a phospho-site in other organisms. However, the comparison of the amino acid sequences and the crystal structures of Asc1p and its orthologues reveals that the phospho-sites S122 of A. thaliana RACK1A, S146 of human RACK1, and S148 of $S$. pombe Cpc2p are located in the same region of the protein (Figure 29, Liu et al., 2007b; Wilson-Grady et al., 2008; Urano et al., 2015). Putative 
phosphorylation of human RACK1 at S146 was proposed to be a prerequisite for dimer formation in vivo and consequently for a RACK1-mediated degradation of the $\alpha$-subunit of the transcription factor HIF-1 (Figure 7, Liu et al., 2007b). So far, there is no evidence for the in vivo homodimerization of Asc1p, but a crystal structure of an Asc1p homodimer could be resolved using a protein sample derived from recombinant expression in E. coli (Yatime et al., 2011). Hence, phosphorylation of Asc1p does not seem to be essential for homodimer formation. However, the yeast phosphorylation site T143 not only lies in close proximity of human S146 (see superposition view of the crystal structures in Figure 29), but also in the center of strand 3 $3 \mathrm{D}$ that is directly positioned at the dimer interface (Yatime et al., 2011). Since S146 is not conserved in S. cerevisiae Asc1p, phosphorylation of T143 of Asc1p could instead regulate a putative homodimer formation of the $\beta$-propeller protein.

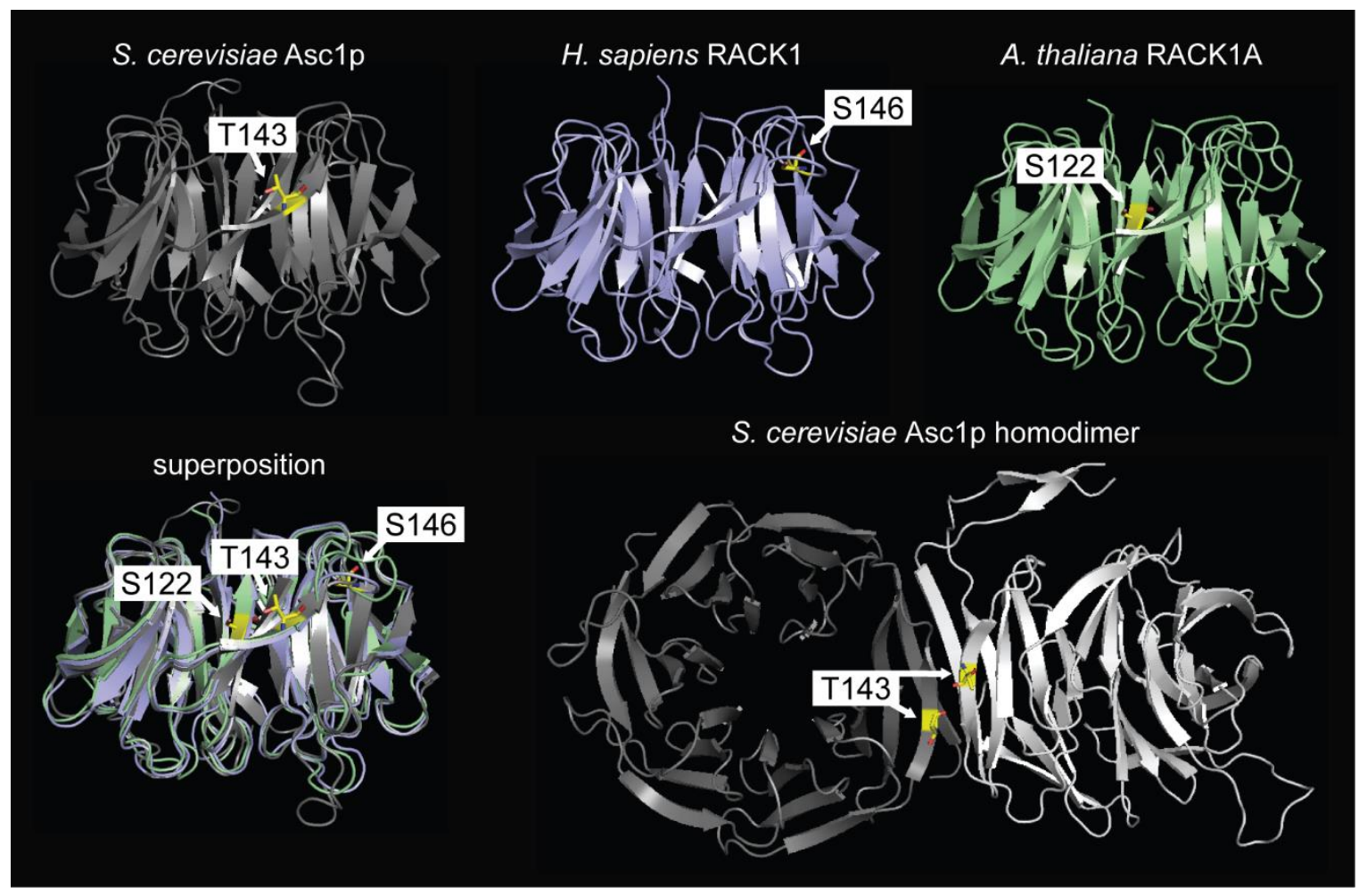

Figure 29. Phosphorylation in the blade 3 region of Asc1p/RACK1.

Structures of $S$. cerevisiae Asc1p (gray), H. sapiens RACK1 (blue), and A. thaliana RACK1A (green) are shown with their respective phosphorylation sites T143, S146, and S122 highlighted. Phosphosites are depicted as sticks with carbon atoms in yellow, nitrogen atoms in blue, and oxygen atoms in red. Additionally, a superposition view of all three structures is depicted. The Asc1p homodimer is displayed with the two interacting Asc1p molecules in gray and white and with the T143 residues from both proteins highlighted. Structure figures were generated with the PyMOL Molecular Graphics System software on the basis of the PDB files 3FRX (Asc1p, Coyle et al., 2009), 4AOW (hRACK1, Ruiz Carrillo et al., 2012), 3DM0 (AtRACK1, Ullah et al., 2008), and 3RFG (Asc1p dimer, Yatime et al., 2011). 
The substitution of T143 for alanine in combination with the DE mutation resulted in a drastic reduction of Asc1 protein levels and significantly changed the phenotypes of the mutant strain. Phosphorylation of Asc1p might regulate the protein's stability as it was proposed for phosphorylation of S122 of A. thaliana RACK1A by WNK8 (Urano et al., 2015). Since S122 of A. thaliana RACK1A lies in close proximity to T143 of S. cerevisiae Asc1p (see the superposition view of the structure in Figure 29), the phosphorylation state of this region may determine the stability of the protein. Not only the amino acid exchange of T143 but also that of T12 and T96 resulted in significant reduction of Asc1 protein levels when combined with the DE mutation. In contrast, the DE mutation itself resulted in increased levels of Asc1p. Furthermore, the additional mutation of T99 to alanine, but not to glutamate, enhanced the effect of the T96A DE mutation on protein levels and certain phenotypes. It is important to note, that neither one of the phospho-site mutations at T12, T96, T99, and T143 alone changed protein levels of Asc1p. Apparently, these residues are all located near the Asc1pribosome binding interface in contrast to S120, S166, T168, and Y250 (Figure 14). Compromised association of Asc1p to the ribosome or even changes in its precise positioning might expose these residues and make them accessible for other proteins. The phosphorylation of T12, T96, T99, or T143 might regulate the integrity of Asc1p as a ribosomal protein and might thereby amplify the ribosome-repulsing effect of the DE mutation. The loss of ribosome-association of Asc1p or its increase in undesired interactions with other proteins could eventually lead to increased degradation rates for the protein.

\subsection{Absence of Asc1p significantly changes the phospho-proteome of $S$. cerevisiae}

Asc1p and its mammalian orthologue RACK1 have emerged as key factors in signal transduction pathways that adjust the phospho-proteome to cellular needs. Here, reduced abundance of the phosphorylated and active form of the MAPK Hog1p of the high osmolarity response pathway was observed in the ascl $^{-}$strain. A reduced phosphorylation of the metazoan orthologue of Hog1p, p38, upon depletion of RACK1 has been reported as well (Lin et al., 2015; Wang et al., 2015b). Accordingly, overexpression of RACK1 was shown to induce p38 phosphorylation. RACK1 seems to mediate the activation of the MAPK through the interaction with upstream MAP2Ks of p38 (Figure 6, Lin et al., 2015; Wang et al., 2015b). In S. cerevisiae, an interaction between Asc1p and the p38-activating MAP2K homologue, Pbs2p, has not been described so far. However, Asc1p associates with the MAP4K Ste20p that acts not only upstream of Hog1p but also of the MAPKs Kss1p and Fus3p, and increased activation of Kss1p through phosphorylation has been detected as well in Asc1p-deficient 
cells (Figure 3, Zeller et al., 2007). In D. melanogaster, a direct interaction between p38b and RACK1 was observed, and RACK1 even seems to be a target of p38b-mediated phosphorylation (Belozerov et al., 2014).

Besides the impact of Asc1p on the phosphorylation status of MAPKs (Chasse et al., 2006; Zeller et al., 2007), the translation factors eIF $2 \alpha$ and eIF4A and a component of the ribosomeassociated complex, Ssz1p, were also reported to harbor Asc1p-sensitive phosphorylation sites (Valerius et al., 2007). Here, SILAC quantification through high-resolution massspectrometry was used to get a comprehensive overview of Asc1p-sensitive phosphorylation sites of proteins in S. cerevisiae. The increased phosphorylation of eIF4A and of Ssz1p was confirmed. Previously, the position of the affected phosphorylation site could be determined only for Ssz1p (Valerius et al., 2007). Here, increased phosphorylation of S2 within eIF4A was observed. Besides eIF4A and Ssz1p, more than 200 proteins were identified to be Asc1pdependently altered in their phosphorylation status at almost 300 different sites. This finding characterizes Asc1p as a key regulator within the cellular phosphorylation network.

\subsection{Versatile Asc1p-dependent changes in the phospho-proteome: How does Asc1p act?}

How does Asc1p affect the phosphorylation status of so many proteins? The scaffold protein might position kinases and/or phosphatases in the proximity to their targets for their subsequent modification as it was shown for mammalian RACK1 that positions the activated PKC $\beta$ II at the ribosome for the phosphorylation of eIF6 and eIF4E (Ceci et al., 2003; Ruan et al., 2012). Here, ribosome-associated proteins with Asc1p-sensitive phosphorylation sites were identified that could depend on a similar regulatory mechanism, among them, for instance, ten translation initiation factors with three subunits of eIF3, namely Rpg1p, Prt1p, and Tif35p. The b-subunit of eIF3, Prt1p is one of 15 proteins with Asc1p-sensitive phosphorylation sites that have been described as physical interaction partners of Asc1p according to the SGD (Figure 22B) and the Asc1p-Strep co-purification experiments presented here. Asc1p was also reported to interact with the c-subunit of eIF3 confirming a physical proximity of Asc1p to eIF3 at ribosomes (Kouba et al., 2012).

The list of proposed physical Asc1p interaction partners comprises the MAP4K Ste20p, which acts upstream of the MAPKs Kss1p, Fus3p, and Hog1p (Zeller et al., 2007). Ste20p contains three phospho-sites that are up-regulated in $a s c 1^{-}$cells and are possibly involved in the regulation of Ste20p's general kinase activity or target/pathway specificity. Asc1p was suggested to inhibit the kinase activity of Ste20p (Zeller et al., 2007). Thus, Asc1p could not only position kinases at their cellular target sites, but also affect their activity. 
In line with an Asc1p-dependent regulation of Ste20p, increased phosphorylation of Myo3p at the Ste20p target site S357 was detected in the absence of Asc1p. Phosphorylation of Myo3p at S357 through Ste20p was shown in vitro and seems to be required for the protein's function (Wu et al., 1997). Furthermore, increased phosphorylation of residues T82 and T83 of the MAPK-target Dig2p was observed. Together with Dig1p, it represses the activity of the transcription factor Ste12p through direct interaction (Figure 3). Upon phosphorylation by MAPKs Kss1p and/or Fus3p, Dig2p, and Dig1p are released from the complex leading to the activation of Ste12p (Tedford et al., 1997). However, it has not been addressed whether the phosphorylation status of T82 and T83 is involved in the MAPK-dependent regulation of Dig2p.

Besides Ste20p, 42 additional proteins involved in signal transduction processes were identified with Asc1p-sensitive phosphorylation sites including also other protein kinases like Cdc28p and Sch9p. In the Asc1p-deficient strain, reduced phosphorylation of residues Y19 of Cdc28p and S726 of Sch9p was detected. These sites are known to be involved in the regulation of the catalytic activities of the kinases (Booher et al., 1993; Urban et al., 2007). The cyclin-dependent kinase Cdc28p coordinates cell cycle progression through phosphorylation of various targets. Phosphorylation of Cdc28p at Y19 by the Swe1p kinase provides one of the mechanisms to regulate the catalytic activity of Cdc28p. Phosphorylation of the kinase at this residue is highly conserved and leads to its inhibition and subsequent entry into mitosis (reviewed in Enserink and Kolodner, 2010). In S. pombe, the absence of the Asc1p orthologue Cpc2p leads to a defect in $\mathrm{G}_{2} / \mathrm{M}$ transition, possibly caused by an increased abundance of the Swe1p-homologous kinase Wee1p and decreased levels of the Weelpinhibitor Cdr2p. In line with this, phosphorylation of the Cdc28p-homologue Cdc2p at Y15 (equivalent to Y19 in S. cerevisiae) showed delayed kinetics during the cell cycle (Nuñez et al., 2010). In this study, increased protein levels of Cdc28p were also observed that have not been detected for Cdc2p in S. pombe. Mammalian RACK1 was described to affect cell cycle progression at the stage of $\mathrm{G}_{1} / \mathrm{S}$ transition through its inhibitory effect on Src kinase activity (Mamidipudi et al., 2004). Collectively, these data suggest that Asc1p and its orthologues influence cell cycle progression.

The Sch9p kinase is phosphorylated by the TOR complex $\underline{1}$ (TORC1) at six residues in its C-terminal domain including S726, which was identified here to be Asc1p-dependently regulated (Urban et al., 2007). TORC1 is one of two complexes that contain the Target of rapamycin Tor1/2 kinase as catalytic subunit and that regulate cellular growth in response to nutrient signals (Loewith et al., 2002). Sch9p is required for the TORC1-mediated regulation 
of ribosome-biosynthesis, translation initiation, and entry into stationary phase. Phosphorylation of the C-terminal domain of Sch9p is required for the protein's activity and is abolished upon rapamycin treatment and carbon or nitrogen starvation (Urban et al., 2007). In this study, phosphorylation of S726 was found to be decreased in ascl $^{-}$cells.

Considering Asc1p-dependent regulation of specific kinases, such as Ste20p, Cdc28p, and Sch9p, the motif-x software tool was applied to find overrepresented kinase motifs surrounding the regulated phospho-sites (localization probability $\geq 0.75$, Schwartz and Gygi, 2005; Chou and Schwartz, 2011). 38\% of the serine and $43 \%$ of the threonine residues were directly followed by a proline residue (Figure 30). Such SP or TP motifs are preferred target sites for MAPKs and cyclin-dependent kinases (Mok et al., 2011). However, these motifs were also high abundant - albeit less frequent - among all phospho-sites identified here by LC-MS irrespective of their SILAC-ratios (31\% SP and 30\% TP). Furthermore, $13 \%$ of all regulated serines were followed by a glutamate at the third C-terminal position, yet, $15 \%$ of all identified phospho-sites harbored this SxxE motif excluding a specific enrichment of this motif. As expected from the many kinases affected by Asc1p, rather a phosphorylation network than a specific motif and its respective kinase are subject to Asc1p-dependent regulation.

The following paragraphs will focus on the impact of Asc1p on the regulation of specific cellular processes. Several candidates of the Asc1p-sensitive phospho-proteome and their associated biological processes will be discussed considering known or putative relations to Asc1p and further taking into account the results obtained from the Asc1p-Strep co-purification experiments.

Asc1p-dependently regulated phosphorylation sites:
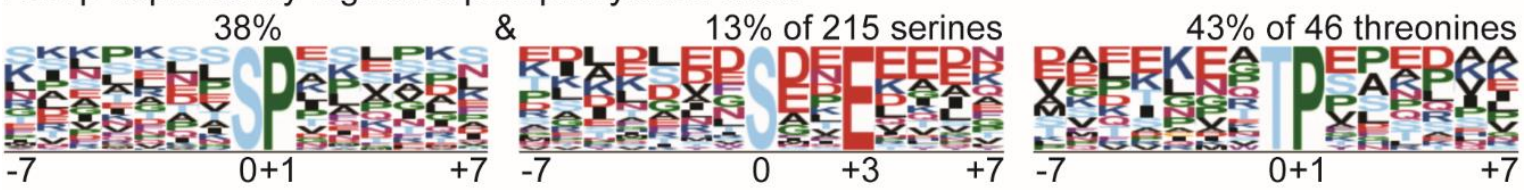

All identified phosphorylation sites:

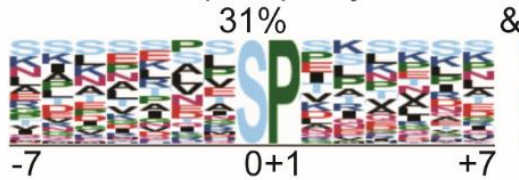

\& $\quad 15 \%$ of 1278 serines
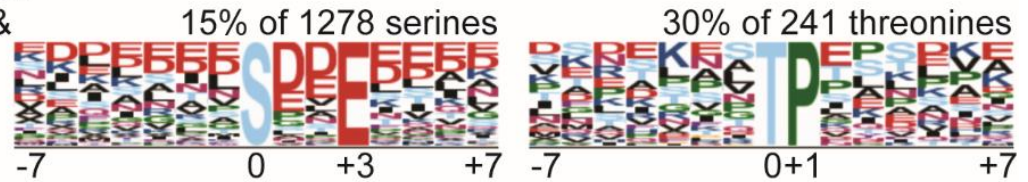

Figure 30. Overrepresented motifs for Asc1p-sensitive phosphorylated serine and threonine residues.

Abundances of motifs for Asc1p-dependently regulated phospho-sites were compared with the occurrence of the same motifs for all phospho-sites identified in this study. Motifs were searched for sites with a localization probability $\geq 0.75$ using the motif- $x$ software (Schwartz and Gygi, 2005; Chou and Schwartz, 2011). Additional motifs identified for the complete phospho-proteome are not depicted. 


\subsection{Asc1p affects localized mRNA translation and cytoskeleton organization}

Among the 213 proteins identified here with Asc1p-sensitive phospho-sites, 42 are involved in processes related to mRNA translation comprising, for example, eIF3 subunits, eIF4A, and Ssz1p. Another protein of this group is the mRNA-binding protein Puf3 with S86 as one of the strongest down-regulated phosphorylation sites in the asc $1^{-}$strain. Puf3p associates with mRNAs that encode mitochondrial proteins and promotes their localized translation at mitochondria (Saint-Georges et al., 2008). Yet, Puf3p was also reported to support the degradation of its bound transcripts (Olivas and Parker, 2000). These opposing roles of Puf3p in mRNA translation and degradation were suggested to be regulated by its phosphorylation (Lee and Tu, 2015). Upon glucose deprivation, Puf3p gets phosphorylated at several residues within its N-terminal region - including the Asc1p-sensitive phospho-site S86 - and promotes the translation of bound transcripts instead of their degradation (Lee and Tu, 2015). In line with the reduced phosphorylation of Puf3p at S86 in asc $1^{-}$cells, our quantitative proteome data revealed that Asc1p-deficiency causes decreased abundance of many mitochondrial proteins, such as ribosomal proteins and subunits of the transporter complexes of the outer and inner mitochondrial membrane (Supplementary Table 15). This regulation of mitochondrial proteins is consistent with the reduced respiratory activity of ascl- cells and their compromised growth on non-fermentable carbon sources (Rachfall et al., 2013).

Reduced phosphorylation was also observed for residue S231 of the mRNA-binding protein Ssd1 in Asc1p-deficient cells. Additionally, Ssd1p was co-purified together with Asc1p-Strep. Ssd1p suppresses the translation of its associated mRNAs until it gets phosphorylated by the kinase Cbk1p at eight residues (Jansen et al., 2009). Many Ssd1p-bound transcripts code for cell wall remodeling proteins. Cbk1p seems to activate the translation of Ssd1p-bound transcripts at sites of cell growth and cell wall remodeling, and disturbance of this regulation leads to defects in cell wall organization. The Asc1p-sensitive residue S231 of Ssd1p has not yet been reported to be involved in the Cbk1p-mediated inhibition of Ssd1p, but lies in close proximity to S228, one of the known target sites of Cbk1p (Jansen et al., 2009). Both the interaction of Ssd1p with Asc1p-Strep and the identification of an Asc1p-sensitive phosphosite within the mRNA-binding protein hint to a functional relationship between the two proteins. This interaction might contribute to the observed defects of Asc1p-deficient cells in cell wall integrity (Valerius et al., 2007; Rachfall et al., 2013).

Asc1p might further be involved in the regulation of specific transcripts through physical association with the mRNA-binding proteins She2 and Scp160 (this study; Baum et al., 2004). Here, She2p was co-purified with Asc1p-Strep. She2p is required for the localization of ASHI 
mRNA to the bud during cell division (Jansen et al., 1996). In the daughter cell, the transcription repressor Ash1p prevents mating type switching (Sil and Herskowitz, 1996). To mediate the asymmetric localization of the ASH1 mRNA, She2p first has to translocate to the nucleus where it is co-transcriptionally recruited to RNA polymerase II by the universally conserved transcription factor heterodimer Spt4p-Spt5p and subsequently binds the nascent ASH1 mRNA (Shen et al., 2010). In the cytoplasm, a transport mRNP is formed through the interaction between She2p and the Myo4p-adaptor protein She3 (Böhl et al., 2000). The mRNP moves along the actin cytoskeleton to the bud tip, and translation is suppressed through the presence of Puf6p and Khd1p in the mRNP (Irie et al., 2002; Gu et al., 2004). When the destination of the ASHI mRNA is reached, phosphorylation of Puf6p and Khd1p triggers the translation of ASH1 mRNA (Paquin et al., 2007; Deng et al., 2008). Besides the ASH1 mRNA, She2p is required for the localization of more than 30 transcripts (reviewed in Heym and Niessing, 2012). Most of these mRNAs contain cis-elements, known as zip-code elements that were shown to be required for the recognition through She2p (Heym and Niessing, 2012). She2p shows similarities to the mammalian mRNA-binding protein ZBP1 (Zipcode-binding protein 1). Remarkably, ZBP1 interacts with RACK1 in transport mRNPs in neuronal cells to regulate the localized translation of $\beta$-actin mRNA (Ceci et al., 2012; Heym and Niessing, 2012). Thus, Asc1p and RACK1 are both involved in mRNA transport. The involvement of Asc1p in She2p directed mRNA localization is further supported by the co-purification of the already mentioned She2p-interacting transcription factor Spt5p with Asc1p-Strep and Asc1DEp-Strep. The Spt4p-Spt5p heterodimer is involved in the elongation process of RNA polymerase II transcription, but has also been found to function in RNA polymerase I-mediated transcription, mRNA capping, and splicing (Hartzog et al., 1998; Wen and Shatkin, 1999; Lindstrom et al., 2003; Schneider et al., 2006). Additionally, the cyclin of the Bur1p kinase, Bur2p (Yao et al., 2000), was co-purified with Asc1DEp-Strep. The Bur1pBur2p complex phosphorylates Spt5p in its C-terminal region, which is required for transcription elongation and histone modification (Liu et al., 2009; Zhou et al., 2009).

Asc1p further interacts with the mRNA-binding protein Scp160 (Baum et al., 2004). Binding of Scp160p to ribosomes requires Asc1p, and as part of a multiprotein complex called SESA network, both proteins regulate the translation of the POM34 mRNA (Baum et al., 2004; Sezen et al., 2009). Like She2p, Scp160p is involved in mRNA transport through targeting of pheromone-induced mRNAs to the shmoo tip during mating (Gelin-Licht et al., 2012). Scp160p was described as an effector protein of Gpalp, the G $\alpha$-protein of the pheromoneresponse pathway (Guo et al., 2003). Through the interaction with Scp160p, Asc1p might link 
the pheromone response pathway to nutrient sensing based on its proposed function as the $\mathrm{G} \beta$-protein for Gpa2p. A role of Asc1p in the pheromone signal transduction pathway has been previously proposed based on the already mentioned interaction of Asc1p with the MAP4K Ste20p and increased activation of the downstream MAPK Kss1p (Zeller et al., 2007). Furthermore, Asc1p-deficient MATa cells exhibit increased sensitivity to the $\alpha$-factor pheromone (Chasse et al., 2006). Altogether, Asc1p seems to be involved in translational regulation through interaction with mRNA-binding proteins. This regulation might not only involve Asc1p-dependent recruitment of mRNA-binding proteins to the ribosome as it was shown for Scp160p but also Asc1p-dependent mRNA transport for localized translation as it was shown for its mammalian orthologue (Baum et al., 2004; Ceci et al., 2012). Furthermore, Asc1p affects the phosphorylation status of mRNA-binding proteins and consequently most likely the fate of their bound transcripts.

Cellular localization of mRNAs most often involves the actin cytoskeleton and motor proteins. Casolari and colleagues (2012) studied the association of mRNAs with cytoskeletal motor proteins. The type I myosin Myo3p was identified to associate with mRNAs that encode key regulators of actin branching and endocytosis. The motor protein seems to be required for the transport of its associated transcripts to actin patches and/or for their anchoring at these sites (Casolari et al., 2012). In ascl- cells, Myo3p is strongly increased in its phosphorylation at S357. As already discussed, Myo3p is most likely phosphorylated at S357 by the MAP4K Ste20p, whose activity seems to be suppressed by Asc1p (Wu et al., 1997; Zeller et al., 2007).

Besides Myo3p, 27 other proteins with Asc1p-sensitive phospho-sites are related to cytoskeleton-associated processes, and 37 proteins are associated with transport and trafficking. One of the proteins involved in cytoskeleton-associated processes is the formin Bni1p/She5p, which was identified in the initial screen for genes required for the asymmetric localization of ASH1 mRNA (Jansen et al., 1996). Phosphorylation of Bnilp was downregulated upon Asc1p-deficiency at S1889. Yet, is has to be noted that no quantitative proteome value was obtained for the protein. The formins Bnilp and Bnrlp nucleate unbranched actin cables and cytokinetic rings, whereas the formation of actin patches at sites of endocytosis requires the Arp $2 / 3$ complex (reviewed in Goode et al., 2015). The Arp2/3 complex associates with nucleation-promoting factors, such as the already mentioned Myo3/5p and the acting binding protein Abp1 (Evangelista et al., 2000; Goode et al., 2001). Abp1p harbors four sites that are reduced in phosphorylation in $\mathrm{ascl}^{-}$cells, whereas the total protein level of Abp1p is increased by more than 70\%. Abp1p acts together with Aim3p to 
regulate actin filament barbed end elongation in the Arp2/3-nucleated networks (Michelot et al., 2013). Phosphorylation of Aim3p at S843 is enhanced in Asc1p-deficient cells, however, a corresponding quantitative proteome value is missing.

The proteins Bud14 and Kel1 were found to interact with Strep-tagged Asc1p variants in this study. A complex of Bud14p with the Kelch proteins Kel1 and Kel2 regulates the displacement of formin Bnrlp from the growing ends of actin filaments and is consequently required for the normal actin cable architecture (Gould et al., 2014). An interaction between Asc1p and Bud14p was suggested from a high throughput study (Gavin et al., 2002). As an additional component of this complex, Asc1p could be directly involved in the coordination of actin cytoskeleton organization. Bud14p was further described as one of the regulatory subunits of the protein phosphatase Glc7p and is required for the localization of Glc7p at sites of polarized growth (Knaus et al., 2005). The localization of Bud14p to these sites in turn relies on the interaction with the Kelch proteins (Knaus et al., 2005; Gould et al., 2014). The Bud14p-Glc7p complex contributes to the regulation of microtubuli interactions at sites of polarized growth (Knaus et al., 2005). Furthermore, it seems to control post-translational modification of the stress-responsive transcription factor Msn2p in dependence of the Ccr4pNot complex (Lenssen et al., 2005). Two other regulatory subunits of Glc7p (Glc8p and Ypilp) were found to be affected in their phosphorylation status in the asc $1^{-}$cells providing further evidence for a link between Asc1p and Glc7p function. Thus, several changes in the Asc1p-dependent phospho-proteome might be caused by altered Glc7p activity. In conclusion, Asc1p might affect cytoskeleton-related processes through mediating the phosphorylation status of key-components of actin-organization.

\subsection{Asc1p's impact on protein folding and degradation}

Phosphorylations of core components of the $26 \mathrm{~S}$ proteasome are sensitive to Asc1p. Furthermore, the abundance of almost all subunits of the $20 \mathrm{~S}$ core particle and two components of the 19S regulatory particle were increased upon Asc1p-deficiency (Figure 31 and Supplementary Table 16). The proteasome and eIF3 were described to form a supercomplex with the ribosome, elongation factors, tRNA synthetases, and chaperones called the translasome that reveals a close connection of protein synthesis and degradation (Sha et al., 2009). Different co-translational quality control pathways exist that recognize aberrant translation processes and involve the proteasome for degradation of the nascent polypeptide chain (reviewed in Inada, 2013). Asc1p is required for nascent polypeptide-dependent translation arrest that is, for example, caused by polybasic sequences in the growing peptide 
chain. This results in the degradation of the translated mRNA and the nascent polypeptide chain (Kuroha et al., 2010; Brandman et al., 2012; Matsuda et al., 2014).

The correct folding of nascent polypeptides is essential to protect newly synthesized proteins from immediate degradation. In eukaryotic cells, the nascent polypeptide-asssociated complex (NAC) can bind to emerging polypeptides to prevent inappropriate interactions and misfolding (Wang et al., 1995). Moreover, the NAC functions in the co-translational delivery of proteins to mitochondria as well as in the translocation of nascent polypeptide chains to the endoplasmic reticulum mediated by the signal recognition particle (George et al., 2002; Zhang et al., 2012b). The NAC is a highly conserved heterodimer formed by the proteins Egd1 and Egd2 in S. cerevisiae. Here, phosphorylation of Egd1p at T151 was identified as the strongest down-regulated phospho-site in the sscl $^{-}$strain.

A role of Asc1p in co-translational protein folding is deduced from the observation that mammalian RACK1 recruits activated JNK to the ribosome in order to phosphorylate eEF1A and with that to induce association of the translation factor with the newly synthesized peptide chain (Gandin et al., 2013a). The translation elongation factor is involved in the recruitment of aminoacyl-tRNAs to the ribosomes, but it can also mediate the co-translational degradation of nascent polypeptide chains by the proteasome (Hotokezaka et al., 2002; Chuang et al., 2005). In T. brucei, RACK1 was shown to associate with eEF1A (Regmi et al., 2008). Moreover, the mRNA-binding protein and interaction partner of Asc1p, Scp160p, was found

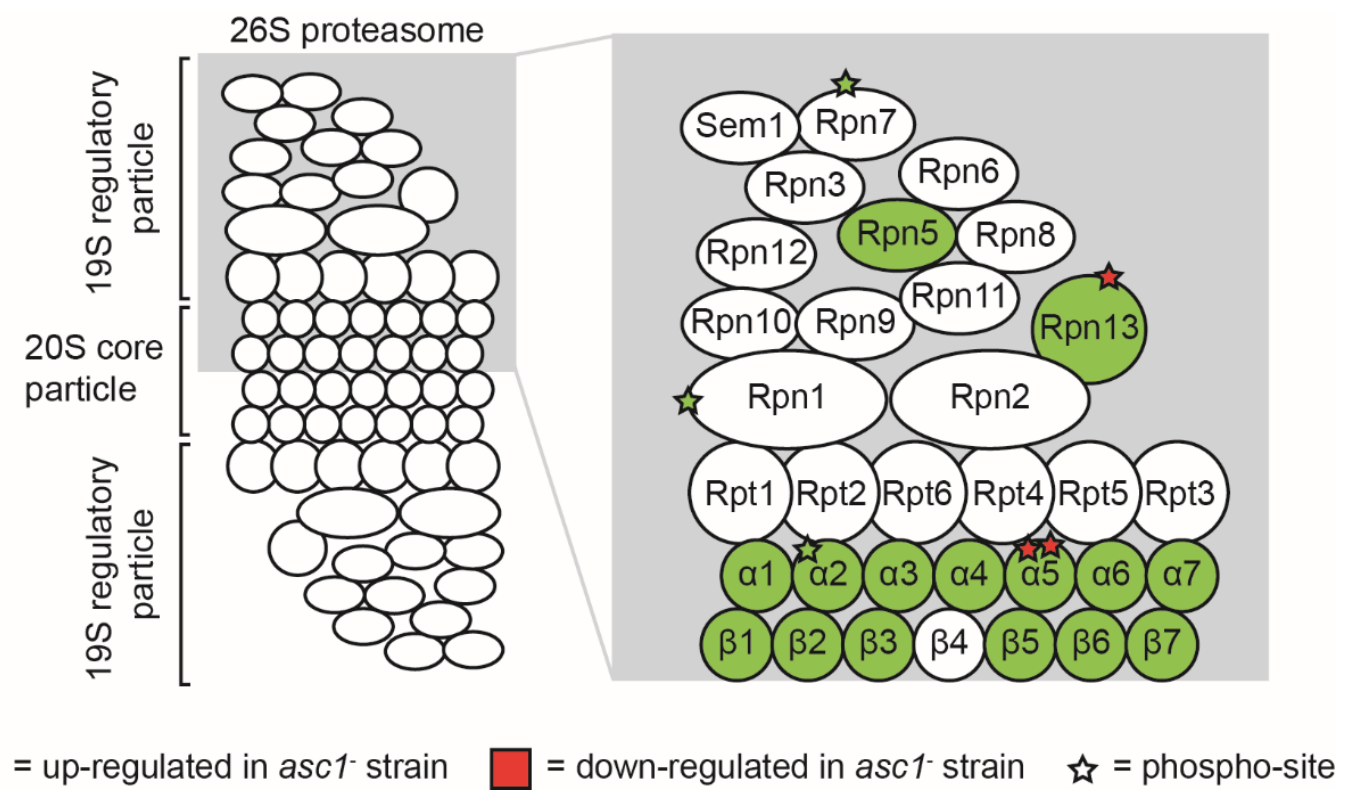

Figure 31. Asc1p-dependent alterations of the 26S proteasome.

Asc1p-sensitive phospho-sites within components of the $26 \mathrm{~S}$ proteasome are indicated with asterisks. Proteins regulated in their abundance in the Asclp-deficient cells are colored. Colors indicate whether the phospho-sites or the proteins were up- or down-regulated in the ascl- strain. The schematic depiction of the $26 \mathrm{~S}$ proteasome was adapted from the KEGG database. 
to associate with eEF1A at ribosomes implicating a physical proximity of eEF1A to Asc1p also in yeast (Baum et al., 2004).

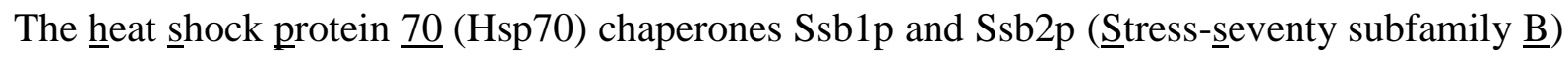
together with the ribosome-associated complex consisting of the Hsp70 Ssz1p and the Hsp40 Zuo1p also assist in the folding of nascent polypeptide chains (Gautschi et al., 2002). Ssz1p contains an Asc1p-sensitive phosphorylation site (this study; Valerius et al., 2007). The family of Hsp70 chaperones further comprises four Ssa (마ress-seventy subfamily $\underline{A}$ ) proteins. Here, Asc1DEp-Strep, but not Asc1p-Strep, was shown to co-purify with Ssa1p and Ssa2p. Ssa1p associates with the Hsp40 Sis1p predominantly on translating ribosomes and both proteins are required for intact mRNA translation (Horton et al., 2001). Although Ssa1p and Ssa2p clearly did not interact with Asc1p-Strep, Sis1p was found enriched with the Streptagged wild-type Asc1 protein. Ssa1/2p and Sis1p are involved in prion propagation (reviewed in Liebman and Chernoff, 2012). The $\left[\mathrm{PSI}^{+}\right]$prion is formed through a refolding of the translation termination factor Sup35p, and deletion of $A S C 1$ results in induction of $\left[\mathrm{PSI}^{+}\right]$ (Manogaran et al., 2011). Like Ssa1/2p, Sup35p was found to interact with Asc1DEp-Strep, but most likely not with Asc1p-Strep. Sup35p has been proposed to function in a process named premature translation termination that releases C-terminally truncated nascent polypeptides from ribosomes stalled on polylysine stretches (Chiabudini et al., 2014). During this process, Sup35p binds to the ribosomal A-site although it does not contain a stop codon. The deletion of ASCl abolishes premature translation termination most likely due to its effect on overall translation rates (Chiabudini et al., 2014). Yet, the data presented here indicate a direct interplay between Asc1p and Sup35p that is further supported by the identification of two Asc1p-sensitive phospho-sites within Sup35p (T570 and S571).

Altogether, Asc1p/RACK1 seems to function in protein folding and degradation during translation and beyond. The involvement of Asc1p in protein degradation processes is further supported by the finding that three proteins with ubiquitin-binding activity namely Def1p, Cue5p, and Dsk2p associate with Asc1p-Strep and/or Asc1DEp-Strep. Both Def1p and Cue5p contain ubiquitin-binding CUE domains (Shih et al., 2003). The CUE domain of Def1p is required for its binding to the ubiquitin-like domain of Ela1p, a component of the ElonginCullin ubiquitin ligase complex. The simultaneous interaction of Def1p with this E3 ubiquitin ligase and the RNA polymerase II results in polyubiquitination of the largest subunit of the polymerase, Rpb1p, and its subsequent degradation (Wilson et al., 2013). The Def1pmediated degradation of RNA polymerase II occurs in response to DNA-damage that causes transcriptional stress. Upon these conditions, Def1p is modified in a ubiquitin- and 
proteasome-dependent process leading to removal of its $\mathrm{C}$-terminal domain and its translocation from the cytoplasm to the nucleus where it mediates Rpb1p degradation (Wilson et al., 2013). Two phosphorylation sites T258 and S260 are reduced in their phosphorylation status in $\mathrm{ascl}^{-}$cells further implicating a functional link between Asc1p and Def1p.

The Cue5 protein was identified as a ubiquitin adaptor protein for the autophagy related protein $\underline{8}$ (Atg8) and thus seems to be involved in substrate recognition for selective macroautophagy (Lu et al., 2014). Dsk2p is involved in the delivery of polyubiquitinated proteins to the proteasome. The protein binds polyubiquitin-chains through its $\mathrm{C}$-terminal ubiquitin-associated domain and interacts with the proteasome through its $\mathrm{N}$-terminal ubiquitin-like domain (Funakoshi et al., 2002). Asc1p was described to be ubiquitinated at ten sites, and it remains to be determined whether the ubiquitin-binding proteins Def1, Cue5, and Dsk2 interact with the ubiquitinated forms of Asc1p-Strep and/or Asc1DEp-Strep (Swaney et al., 2013).

\subsection{The $\beta$-propeller Asc1p represents a regulatory interface connecting mRNA translation with fundamental cellular processes according to signals}

This work focused on the role of Asc1p in the cellular phosphorylation network of S. cerevisiae. The exposed localization of the $\beta$-propeller at the ribosome goes along with an important coordination function that connects a variety of cellular processes to local mRNA translation and signal transduction. It influences the activities of central signal transduction players, such as the kinases PKA, Cdc28p, Ste20p, Kss1p, Slt2p, and Hog1p as well as the phosphatase Glc7p, which coordinate cellular processes like cell cycle progression, maintenance of cell wall integrity, and adaptation to osmolarity and hypoxic growth conditions (Figure 32). Fundamental processes like cytoskeleton organization, protein folding and turnover, and even nuclear transcription processes are significantly affected by the absence of Asc1p. Translation of the transcription factor $Y H R 177 \mathrm{~W}$ mRNA seems highly Asc1p-dependent. This diversified impact of a single protein might be due to its high abundance and presence in almost every ribosome. Especially, different local activity of ribosomes, for example, at mitochondria, could be subject to Asc1p-dependent regulation. The interaction of Asc1p with mRNA-binding proteins or its effect on their phosphorylation status could provide a mechanism for translational regulation. Asc1p is itself phosphorylated at multiple sites. Here, four of these sites were identified to be involved in the regulation of Asc1p's abundance. Absence of Asc1p uncouples the translation machinery from certain signal transduction pathways and limits the ability of the cell to adapt to stress conditions. 


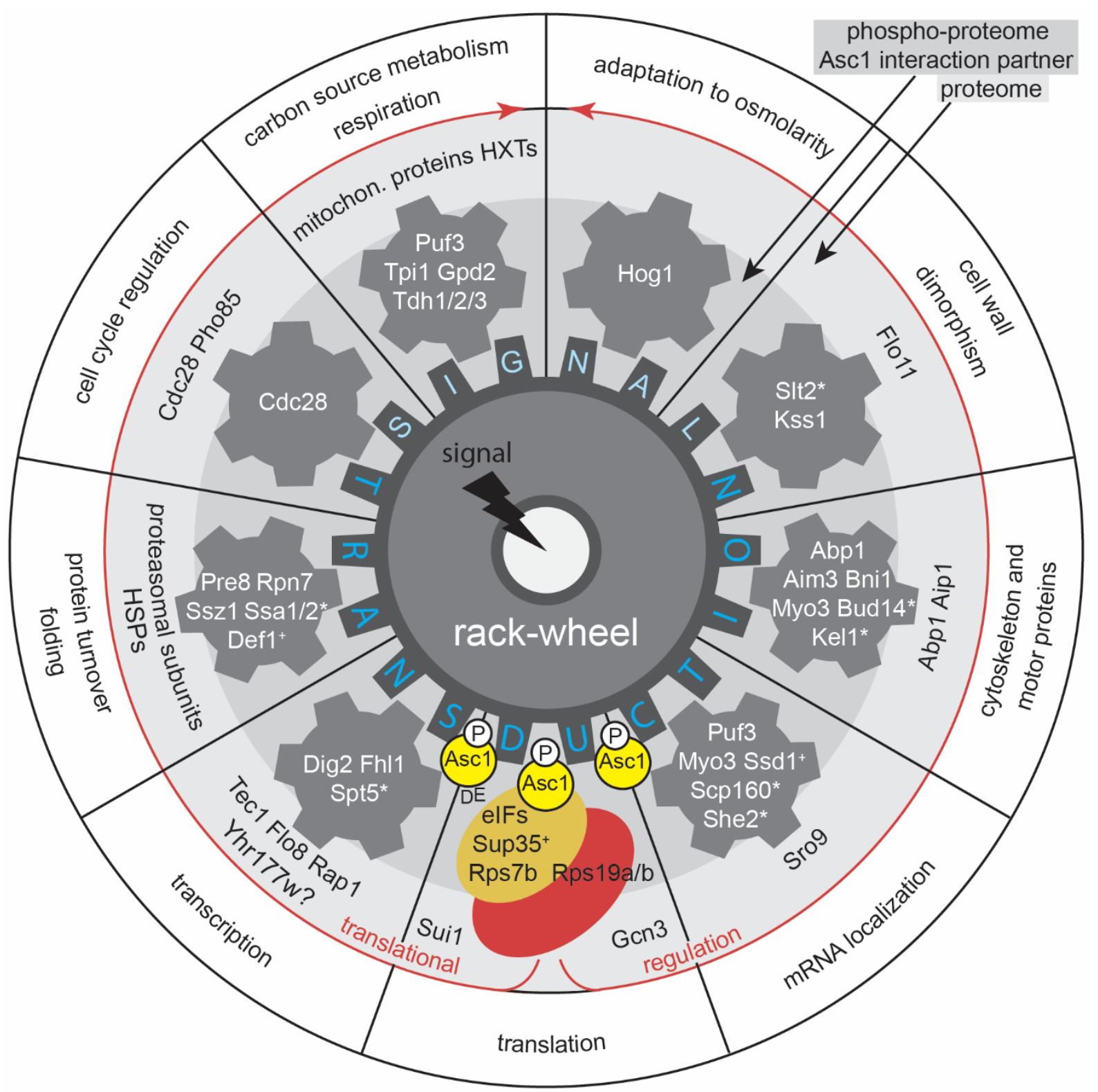

Figure 32. The rack-wheel of Asc1p-dependent signal transduction.

The proposed function of Asc1p in the adaptation of the cellular proteome and phospho-proteome in response to signal transduction is schematically depicted. Asc1p links cellular signal transduction to the ribosome leading to the adjustment of the cellular proteome as needed (indicated by red arrows). Simultaneously, Asc1p is important for the phosphorylation status of central components of various processes named in the outermost ring of the scheme. The inner ring around the central rack-wheel gives examples for proteins containing Asc1p-sensitive phosphorylation sites. Proteins labeled with a plus $\left(^{+}\right)$were additionally identified as interaction partners of Asc1p. Proteins marked with an asterisk (*) were not identified with Asc1p-sensitive phospho-sites but as Asc1p-interacting proteins. In the outer gray ring, examples for proteins are given that are Asc1p-dependently regulated on the proteome level. For the transcription factor Yhr177wp, translation of its encoding mRNA seems to be directly Asc1p-dependent. Also, a ribosome-free form of Asc1p could be part of the signal transduction machinery. Ribosome-association of Asclp can be impaired through the DE mutation. Phosphorylation $(\mathrm{P})$ of Asc1p itself could modulate the protein's integrity as a link between translation and signal transduction. Additional references used apart from this study: Flo11p (Valerius et al., 2007), Kss1p (Zeller et al., 2007), Scp160p (Baum et al., 2004), and Slt2p (Chasse et al., 2006; Breitkreutz et al., 2010). (HSP $=$ heat shock protein, Hxts $=$ hexose transporters) 


\section{References}

Adams, D.R., Ron, D., and Kiely, P.A. (2011). RACK1, A multifaceted scaffolding protein: Structure and function. Cell Commun Signal 9, 22.

Alspaugh, J.A., Perfect, J.R., and Heitman, J. (1997). Cryptococcus neoformans mating and virulence are regulated by the G-protein $\alpha$ subunit GPA1 and cAMP. Genes Dev $11,3206-3217$.

Amir, S., Wang, R., Simons, J.W., and Mabjeesh, N.J. (2009). SEPT9_v1 up-regulates hypoxia-inducible factor 1 by preventing its RACK1-mediated degradation. J Biol Chem 284, 11142-11151.

Arimoto, K., Fukuda, H., Imajoh-Ohmi, S., Saito, H., and Takekawa, M. (2008). Formation of stress granules inhibits apoptosis by suppressing stress-responsive MAPK pathways. Nat Cell Biol 10, 1324-1332.

Balakin, A.G., Smith, L., and Fournier, M.J. (1996). The RNA world of the nucleolus: two major families of small RNAs defined by different box elements with related functions. Cell 86, 823-834.

Bardwell, L. (2005). A walk-through of the yeast mating pheromone response pathway. Peptides 26, 339-350.

Baum, S., Bittins, M., Frey, S., and Seedorf, M. (2004). Asc1p, a WD40-domain containing adaptor protein, is required for the interaction of the RNA-binding protein Scp160p with polysomes. Biochem J 380, 823-830.

Belozerov, V.E., Ratkovic, S., McNeill, H., Hilliker, A.J., and McDermott, J.C. (2014). In vivo interaction proteomics reveal a novel $\mathrm{p} 38$ mitogen-activated protein kinase/Rack1 pathway regulating proteostasis in Drosophila muscle. Mol Cell Biol 34, 474-484.

Ben-Shem, A., Garreau de Loubresse, N., Melnikov, S., Jenner, L., Yusupova, G., and Yusupov, M. (2011). The structure of the eukaryotic ribosome at 3.0 A resolution. Science 334, 1524-1529.

Berns, H., Humar, R., Hengerer, B., Kiefer, F.N., and Battegay, E.J. (2000). RACK1 is upregulated in angiogenesis and human carcinomas. FASEB J 14, 2549-2558.

Bird, R.J., Baillie, G.S., and Yarwood, S.J. (2010). Interaction with receptor for activated Ckinase 1 (RACK1) sensitizes the phosphodiesterase PDE4D5 towards hydrolysis of cAMP and activation by protein kinase C. Biochem J 432, 207-216.

Böhl, F., Kruse, C., Frank, A., Ferring, D., and Jansen, R.P. (2000). She2p, a novel RNAbinding protein tethers $A S H 1 \mathrm{mRNA}$ to the Myo4p myosin motor via She3p. EMBO J $19,5514-5524$.

Booher, R.N., Deshaies, R.J., and Kirschner, M.W. (1993). Properties of Saccharomyces cerevisiae wee1 and its differential regulation of $\mathrm{p} 34^{C D C 28}$ in response to $\mathrm{G}_{1}$ and $\mathrm{G}_{2}$ cyclins. EMBO J 12, 3417-3426.

Brandman, O., Stewart-Ornstein, J., Wong, D., Larson, A., Williams, C.C., Li, G.W., Zhou, S., King, D., Shen, P.S., Weibezahn, J., et al. (2012). A ribosome-bound quality 
control complex triggers degradation of nascent peptides and signals translation stress. Cell 151, 1042-1054.

Breitkreutz, A., Choi, H., Sharom, J.R., Boucher, L., Neduva, V., Larsen, B., Lin, Z.Y., Breitkreutz, B.J., Stark, C., Liu, G., et al. (2010). A global protein kinase and phosphatase interaction network in yeast. Science 328, 1043-1046.

Brown, G.C. (2001). Regulation of mitochondrial respiration by nitric oxide inhibition of cytochrome c oxidase. Biochim Biophys Acta 1504, 46-57.

Burnette, W.N. (1981). "Western blotting": electrophoretic transfer of proteins from sodium dodecyl sulfate--polyacrylamide gels to unmodified nitrocellulose and radiographic detection with antibody and radioiodinated protein A. Anal Biochem 112, 195-203.

Cai, Z.D., Chai, Y.F., Zhang, C.Y., Qiao, W.R., Sang, H., and Lu, L. (2015). The Gß-like protein $\mathrm{CpcB}$ is required for hyphal growth, conidiophore morphology and pathogenicity in Aspergillus fumigatus. Fungal Genet Biol 81, 120-131.

Cain, C.W., Lohse, M.B., Homann, O.R., Sil, A., and Johnson, A.D. (2012). A conserved transcriptional regulator governs fungal morphology in widely diverged species. Genetics 190, 511-521.

Cao, X.X., Xu, J.D., Liu, X.L., Xu, J.W., Wang, W.J., Li, Q.Q., Chen, Q., Xu, Z.D., and Liu, X.P. (2010). RACK1: A superior independent predictor for poor clinical outcome in breast cancer. Int J Cancer 127, 1172-1179.

Casolari, J.M., Thompson, M.A., Salzman, J., Champion, L.M., Moerner, W.E., and Brown, P.O. (2012). Widespread mRNA association with cytoskeletal motor proteins and identification and dynamics of myosin-associated mRNAs in S. cerevisiae. PLoS One 7, e31912.

Castello, P.R., David, P.S., McClure, T., Crook, Z., and Poyton, R.O. (2006). Mitochondrial cytochrome oxidase produces nitric oxide under hypoxic conditions: implications for oxygen sensing and hypoxic signaling in eukaryotes. Cell Metab 3, 277-287.

Ceci, M., Gaviraghi, C., Gorrini, C., Sala, L.A., Offenhäuser, N., Marchisio, P.C., and Biffo, S. (2003). Release of eIF6 (p27 $7^{\mathrm{BBP}}$ ) from the $60 \mathrm{~S}$ subunit allows $80 \mathrm{~S}$ ribosome assembly. Nature 426, 579-584.

Ceci, M., Welshhans, K., Ciotti, M.T., Brandi, R., Parisi, C., Paoletti, F., Pistillo, L., Bassell, G.J., and Cattaneo, A. (2012). RACK1 is a ribosome scaffold protein for $\beta$-actin mRNA/ZBP1 complex. PLoS One 7, e35034.

Chang, B.Y., Chiang, M., and Cartwright, C.A. (2001). The interaction of Src and RACK1 is enhanced by activation of protein kinase $\mathrm{C}$ and tyrosine phosphorylation of RACK1. $\mathrm{J}$ Biol Chem 276, 20346-20356.

Chang, B.Y., Conroy, K.B., Machleder, E.M., and Cartwright, C.A. (1998). RACK1, a receptor for activated $\mathrm{C}$ kinase and a homolog of the $\beta$ subunit of $\mathrm{G}$ proteins, inhibits activity of src tyrosine kinases and growth of NIH 3T3 cells. Mol Cell Biol 18, 3245-3256. 
Chang, B.Y., Harte, R.A., and Cartwright, C.A. (2002). RACK1: a novel substrate for the Src protein-tyrosine kinase. Oncogene 21, 7619-7629.

Chantrel, Y., Gaisne, M., Lions, C., and Verdière, J. (1998). The transcriptional regulator Haplp (Cyp1p) is essential for anaerobic or heme-deficient growth of Saccharomyces cerevisiae: Genetic and molecular characterization of an extragenic suppressor that encodes a WD repeat protein. Genetics 148, 559-569.

Chasse, S.A., Flanary, P., Parnell, S.C., Hao, N., Cha, J.Y., Siderovski, D.P., and Dohlman, H.G. (2006). Genome-scale analysis reveals Sst2 as the principal regulator of mating pheromone signaling in the yeast Saccharomyces cerevisiae. Eukaryot Cell 5, 330346.

Chen, J.G., Ullah, H., Temple, B., Liang, J., Guo, J., Alonso, J.M., Ecker, J.R., and Jones, A.M. (2006). RACK1 mediates multiple hormone responsiveness and developmental processes in Arabidopsis. J Exp Bot 57, 2697-2708.

Chen, S., Dell, E.J., Lin, F., Sai, J., and Hamm, H.E. (2004a). RACK1 regulates specific functions of G $\beta \gamma$. J Biol Chem 279, 17861-17868.

Chen, S., Lin, F., and Hamm, H.E. (2005). RACK1 binds to a signal transfer region of G $\beta \gamma$ and inhibits phospholipase C $\beta 2$ activation. J Biol Chem 280, 33445-33452.

Chen, S., Spiegelberg, B.D., Lin, F., Dell, E.J., and Hamm, H.E. (2004b). Interaction of G $\beta \gamma$ with RACK1 and other WD40 repeat proteins. J Mol Cell Cardiol 37, 399-406.

Cheng, Z., Li, J.F., Niu, Y., Zhang, X.C., Woody, O.Z., Xiong, Y., Djonović, S., Millet, Y., Bush, J., McConkey, B.J., et al. (2015). Pathogen-secreted proteases activate a novel plant immune pathway. Nature 521, 213-216.

Chi, A., Huttenhower, C., Geer, L.Y., Coon, J.J., Syka, J.E., Bai, D.L., Shabanowitz, J., Burke, D.J., Troyanskaya, O.G., and Hunt, D.F. (2007). Analysis of phosphorylation sites on proteins from Saccharomyces cerevisiae by electron transfer dissociation (ETD) mass spectrometry. Proc Natl Acad Sci U S A 104, 2193-2198.

Chiabudini, M., Tais, A., Zhang, Y., Hayashi, S., Wölfle, T., Fitzke, E., and Rospert, S. (2014). Release factor eRF3 mediates premature translation termination on polylysinestalled ribosomes in Saccharomyces cerevisiae. Mol Cell Biol 34, 4062-4076.

Chou, M.F., and Schwartz, D. (2011). Biological sequence motif discovery using motif-x. Curr Protoc Bioinformatics Chapter 13, Unit 13 15-24.

Chou, Y.C., Chou, C.C., Chen, Y.K., Tsai, S., Hsieh, F.M., Liu, H.J., and Hseu, T.H. (1999). Structure and genomic organization of porcine RACK1 gene. Biochim Biophys Acta $1489,315-322$.

Choudhury, K., Cardenas, D., Pullikuth, A.K., Catling, A.D., Aiyar, A., and Kelly, B.L. (2011). Trypanosomatid RACK1 orthologs show functional differences associated with translation despite similar roles in Leishmania pathogenesis. PLoS One 6, e20710. 
Chuang, S.M., Chen, L., Lambertson, D., Anand, M., Kinzy, T.G., and Madura, K. (2005). Proteasome-mediated degradation of cotranslationally damaged proteins involves translation elongation factor 1A. Mol Cell Biol 25, 403-413.

Conti, M., Richter, W., Mehats, C., Livera, G., Park, J.Y., and Jin, C. (2003). Cyclic AMPspecific PDE4 phosphodiesterases as critical components of cyclic AMP signaling. J Biol Chem 278, 5493-5496.

Cox, J., and Mann, M. (2008). MaxQuant enables high peptide identification rates, individualized p.p.b.-range mass accuracies and proteome-wide protein quantification. Nat Biotechnol 26, 1367-1372.

Coyle, S.M., Gilbert, W.V., and Doudna, J.A. (2009). Direct link between RACK1 function and localization at the ribosome in vivo. Mol Cell Biol 29, 1626-1634.

Cross, F.R., and Tinkelenberg, A.H. (1991). A potential positive feedback loop controlling CLN1 and CLN2 gene expression at the start of the yeast cell cycle. Cell 65,875-883.

de Oliveira, I.M., Zanotto-Filho, A., Moreira, J.C., Bonatto, D., and Henriques, J.A. (2010). The role of two putative nitroreductases, Frm2p and Hbn1p, in the oxidative stress response in Saccharomyces cerevisiae. Yeast 27, 89-102.

Decker, C.J., and Parker, R. (2012). P-bodies and stress granules: possible roles in the control of translation and mRNA degradation. Cold Spring Harb Perspect Biol 4, a012286.

Dell, E.J., Connor, J., Chen, S., Stebbins, E.G., Skiba, N.P., Mochly-Rosen, D., and Hamm, H.E. (2002). The $\beta \gamma$ subunit of heterotrimeric G proteins interacts with RACK1 and two other WD repeat proteins. J Biol Chem 277, 49888-49895.

Deng, Y., Singer, R.H., and Gu, W. (2008). Translation of ASH1 mRNA is repressed by Puf6p-Fun12p/eIF5B interaction and released by CK2 phosphorylation. Genes Dev $22,1037-1050$.

Dever, T.E., Feng, L., Wek, R.C., Cigan, A.M., Donahue, T.F., and Hinnebusch, A.G. (1992). Phosphorylation of initiation factor $2 \alpha$ by protein kinase GCN2 mediates genespecific translational control of GCN4 in yeast. Cell 68, 585-596.

Enserink, J.M., and Kolodner, R.D. (2010). An overview of Cdk1-controlled targets and processes. Cell Div 5, 11.

Evangelista, M., Klebl, B.M., Tong, A.H., Webb, B.A., Leeuw, T., Leberer, E., Whiteway, M., Thomas, D.Y., and Boone, C. (2000). A role for myosin-I in actin assembly through interactions with Vrp1p, Bee1p, and the Arp2/3 complex. J Cell Biol 148, 353-362.

Feng, C., Li, Y.F., Yau, Y.H., Lee, H.S., Tang, X.Y., Xue, Z.H., Zhou, Y.C., Lim, W.M., Cornvik, T.C., Ruedl, C., et al. (2012). Kindlin-3 mediates integrin $\alpha \mathrm{L} \beta 2$ outside-in signaling, and it interacts with scaffold protein receptor for activated-C kinase 1 (RACK1). J Biol Chem 287, 10714-10726.

Fomenkov, A., Zangen, R., Huang, Y.P., Osada, M., Guo, Z., Fomenkov, T., Trink, B., Sidransky, D., and Ratovitski, E.A. (2004). RACK1 and stratifin target $\Delta \mathrm{Np} 63 \alpha$ for a 
proteasome degradation in head and neck squamous cell carcinoma cells upon DNA damage. Cell Cycle 3, 1285-1295.

Funakoshi, M., Sasaki, T., Nishimoto, T., and Kobayashi, H. (2002). Budding yeast Dsk2p is a polyubiquitin-binding protein that can interact with the proteasome. Proc Natl Acad Sci U S A 99, 745-750.

Galibert, L., Tometsko, M.E., Anderson, D.M., Cosman, D., and Dougall, W.C. (1998). The involvement of multiple tumor necrosis factor receptor (TNFR)-associated factors in the signaling mechanisms of receptor activator of NF- $\kappa \mathrm{B}$, a member of the TNFR superfamily. J Biol Chem 273, 34120-34127.

Gallina, A., Rossi, F., and Milanesi, G. (2001). Rack1 binds HIV-1 Nef and can act as a Nefprotein kinase C adaptor. Virology 283, 7-18.

Gandin, V., Gutierrez, G.J., Brill, L.M., Varsano, T., Feng, Y., Aza-Blanc, P., Au, Q., McLaughlan, S., Ferreira, T.A., Alain, T., et al. (2013a). Degradation of newly synthesized polypeptides by ribosome-associated RACK1/c-Jun N-terminal kinase/eukaryotic elongation factor 1A2 complex. Mol Cell Biol 33, 2510-2526.

Gandin, V., Senft, D., Topisirovic, I., and Ronai, Z.A. (2013b). RACK1 Function in Cell Motility and Protein Synthesis. Genes Cancer 4, 369-377.

Ganot, P., Caizergues-Ferrer, M., and Kiss, T. (1997). The family of box ACA small nucleolar RNAs is defined by an evolutionarily conserved secondary structure and ubiquitous sequence elements essential for RNA accumulation. Genes Dev 11, 941956.

Gautschi, M., Mun, A., Ross, S., and Rospert, S. (2002). A functional chaperone triad on the yeast ribosome. Proc Natl Acad Sci U S A 99, 4209-4214.

Gavin, A.C., Bösche, M., Krause, R., Grandi, P., Marzioch, M., Bauer, A., Schultz, J., Rick, J.M., Michon, A.M., Cruciat, C.M., et al. (2002). Functional organization of the yeast proteome by systematic analysis of protein complexes. Nature 415, 141-147.

Gelin-Licht, R., Paliwal, S., Conlon, P., Levchenko, A., and Gerst, J.E. (2012). Scp160dependent mRNA trafficking mediates pheromone gradient sensing and chemotropism in yeast. Cell Rep 1, 483-494.

George, R., Walsh, P., Beddoe, T., and Lithgow, T. (2002). The nascent polypeptideassociated complex (NAC) promotes interaction of ribosomes with the mitochondrial surface in vivo. FEBS Lett 516, 213-216.

Gerbasi, V.R., Weaver, C.M., Hill, S., Friedman, D.B., and Link, A.J. (2004). Yeast Asc1p and mammalian RACK1 are functionally orthologous core 40S ribosomal proteins that repress gene expression. Mol Cell Biol 24, 8276-8287.

Gnad, F., de Godoy, L.M., Cox, J., Neuhauser, N., Ren, S., Olsen, J.V., and Mann, M. (2009). High-accuracy identification and bioinformatic analysis of in vivo protein phosphorylation sites in yeast. Proteomics 9, 4642-4652. 
Goode, B.L., Eskin, J.A., and Wendland, B. (2015). Actin and endocytosis in budding yeast. Genetics 199, 315-358.

Goode, B.L., Rodal, A.A., Barnes, G., and Drubin, D.G. (2001). Activation of the Arp2/3 complex by the actin filament binding protein Abp1p. J Cell Biol 153, 627-634.

Gould, C.J., Chesarone-Cataldo, M., Alioto, S.L., Salin, B., Sagot, I., and Goode, B.L. (2014). Saccharomyces cerevisiae Kelch proteins and Bud14 protein form a stable 520-kDa formin regulatory complex that controls actin cable assembly and cell morphogenesis. J Biol Chem 289, 18290-18301.

Grosso, S., Volta, V., Sala, L.A., Vietri, M., Marchisio, P.C., Ron, D., and Biffo, S. (2008). PKC $\beta$ II modulates translation independently from mTOR and through RACK1. Biochem J 415, 77-85.

Gu, W., Deng, Y., Zenklusen, D., and Singer, R.H. (2004). A new yeast PUF family protein, Puf6p, represses ASH1 mRNA translation and is required for its localization. Genes Dev 18, 1452-1465.

Gueldener, U., Heinisch, J., Koehler, G.J., Voss, D., and Hegemann, J.H. (2002). A second set of loxP marker cassettes for Cre-mediated multiple gene knockouts in budding yeast. Nucleic Acids Res 30, e23.

Guo, J., and Chen, J.G. (2008). RACK1 genes regulate plant development with unequal genetic redundancy in Arabidopsis. BMC Plant Biol 8, 108.

Guo, J., Wang, J., Xi, L., Huang, W.D., Liang, J., and Chen, J.G. (2009). RACK1 is a negative regulator of ABA responses in Arabidopsis. J Exp Bot 60, 3819-3833.

Guo, J., Wang, S., Valerius, O., Hall, H., Zeng, Q., Li, J.F., Weston, D.J., Ellis, B.E., and Chen, J.G. (2011). Involvement of Arabidopsis RACK1 in protein translation and its regulation by abscisic acid. Plant Physiol 155, 370-383.

Guo, M., Aston, C., Burchett, S.A., Dyke, C., Fields, S., Rajarao, S.J., Uetz, P., Wang, Y., Young, K., and Dohlman, H.G. (2003). The yeast G protein $\alpha$ subunit Gpa1 transmits a signal through an RNA binding effector protein Scp160. Mol Cell 12, 517-524.

Guo, Y., Wang, W., Wang, J., Feng, J., Wang, Q., Jin, J., Lv, M., Li, X., Li, Y., Ma, Y., et al. (2013). Receptor for activated C kinase 1 promotes hepatocellular carcinoma growth by enhancing mitogen-activated protein kinase kinase 7 activity. Hepatology 57, 140151.

Hardcastle, T.J., and Kelly, K.A. (2010). baySeq: empirical Bayesian methods for identifying differential expression in sequence count data. BMC Bioinformatics 11, 422.

Hartzog, G.A., Wada, T., Handa, H., and Winston, F. (1998). Evidence that Spt4, Spt5, and Spt6 control transcription elongation by RNA polymerase II in Saccharomyces cerevisiae. Genes Dev 12, 357-369.

He, D.Y., Neasta, J., and Ron, D. (2010). Epigenetic regulation of BDNF expression via the scaffolding protein RACK1. J Biol Chem 285, 19043-19050. 
Helser, T.L., Baan, R.A., and Dahlberg, A.E. (1981). Characterization of a 40S ribosomal subunit complex in polyribosomes of Saccharomyces cerevisiae treated with cycloheximide. Mol Cell Biol 1, 51-57.

Henriksen, P., Wagner, S.A., Weinert, B.T., Sharma, S., Bacinskaja, G., Rehman, M., Juffer, A.H., Walther, T.C., Lisby, M., and Choudhary, C. (2012). Proteome-wide analysis of lysine acetylation suggests its broad regulatory scope in Saccharomyces cerevisiae. Mol Cell Proteomics 11, 1510-1522.

Hermanto, U., Zong, C.S., Li, W., and Wang, L.H. (2002). RACK1, an insulin-like growth factor I (IGF-I) receptor-interacting protein, modulates IGF-I-dependent integrin signaling and promotes cell spreading and contact with extracellular matrix. Mol Cell Biol 22, 2345-2365.

Heym, R.G., and Niessing, D. (2012). Principles of mRNA transport in yeast. Cell Mol Life Sci 69, 1843-1853.

Hoffman, C.S., and Winston, F. (1987). A ten-minute DNA preparation from yeast efficiently releases autonomous plasmids for transformation of Escherichia coli. Gene 57, 267272.

Hoffmann, B., Wanke, C., Lapaglia, S.K., and Braus, G.H. (2000). c-Jun and RACK1 homologues regulate a control point for sexual development in Aspergillus nidulans. Mol Microbiol 37, 28-41.

Holt, L.J., Tuch, B.B., Villen, J., Johnson, A.D., Gygi, S.P., and Morgan, D.O. (2009). Global analysis of Cdk1 substrate phosphorylation sites provides insights into evolution. Science 325, 1682-1686.

Horton, L.E., James, P., Craig, E.A., and Hensold, J.O. (2001). The yeast hsp70 homologue Ssa is required for translation and interacts with Sis1 and Pab1 on translating ribosomes. J Biol Chem 276, 14426-14433.

Hotokezaka, Y., Tobben, U., Hotokezaka, H., Van Leyen, K., Beatrix, B., Smith, D.H., Nakamura, T., and Wiedmann, M. (2002). Interaction of the eukaryotic elongation factor 1A with newly synthesized polypeptides. J Biol Chem 277, 18545-18551.

Huang da, W., Sherman, B.T., and Lempicki, R.A. (2009a). Bioinformatics enrichment tools: paths toward the comprehensive functional analysis of large gene lists. Nucleic Acids Res 37, 1-13.

Huang da, W., Sherman, B.T., and Lempicki, R.A. (2009b). Systematic and integrative analysis of large gene lists using DAVID bioinformatics resources. Nat Protoc 4, 4457.

Huang, G., Wang, H., Chou, S., Nie, X., Chen, J., and Liu, H. (2006). Bistable expression of WOR1, a master regulator of white-opaque switching in Candida albicans. Proc Natl Acad Sci U S A 103, 12813-12818.

Hunter, T., and Plowman, G.D. (1997). The protein kinases of budding yeast: six score and more. Trends Biochem Sci 22, 18-22. 
Inada, T. (2013). Quality control systems for aberrant mRNAs induced by aberrant translation elongation and termination. Biochim Biophys Acta 1829, 634-642.

Inada, T., Winstall, E., Tarun, S.Z., Jr., Yates, J.R., 3rd, Schieltz, D., and Sachs, A.B. (2002). One-step affinity purification of the yeast ribosome and its associated proteins and mRNAs. RNA 8, 948-958.

Inoue, H., Nojima, H., and Okayama, H. (1990). High efficiency transformation of Escherichia coli with plasmids. Gene 96, 23-28.

Irie, K., Tadauchi, T., Takizawa, P.A., Vale, R.D., Matsumoto, K., and Herskowitz, I. (2002). The Khd1 protein, which has three KH RNA-binding motifs, is required for proper localization of ASH1 mRNA in yeast. EMBO J 21, 1158-1167.

Ishida, S., Takahashi, Y., and Nagata, T. (1993). Isolation of cDNA of an auxin-regulated gene encoding a $\mathrm{G}$ protein $\beta$ subunit-like protein from tobacco BY-2 cells. Proc Natl Acad Sci U S A 90, 11152-11156.

Ito, H., Fukuda, Y., Murata, K., and Kimura, A. (1983). Transformation of intact yeast cells treated with alkali cations. J Bacteriol 153, 163-168.

Ivan, M., Kondo, K., Yang, H., Kim, W., Valiando, J., Ohh, M., Salic, A., Asara, J.M., Lane, W.S., and Kaelin, W.G., Jr. (2001). HIF $\alpha$ targeted for VHL-mediated destruction by proline hydroxylation: implications for $\mathrm{O}_{2}$ sensing. Science 292, 464-468.

Iyer, N.V., Kotch, L.E., Agani, F., Leung, S.W., Laughner, E., Wenger, R.H., Gassmann, M., Gearhart, J.D., Lawler, A.M., Yu, A.Y., et al. (1998). Cellular and developmental control of $\mathrm{O} 2$ homeostasis by hypoxia-inducible factor $1 \alpha$. Genes Dev 12, 149-162.

Jaakkola, P., Mole, D.R., Tian, Y.M., Wilson, M.I., Gielbert, J., Gaskell, S.J., von Kriegsheim, A., Hebestreit, H.F., Mukherji, M., Schofield, C.J., et al. (2001). Targeting of HIF- $\alpha$ to the von Hippel-Lindau ubiquitylation complex by $\mathrm{O}_{2}$ regulated prolyl hydroxylation. Science 292, 468-472.

Janke, C., Magiera, M.M., Rathfelder, N., Taxis, C., Reber, S., Maekawa, H., MorenoBorchart, A., Doenges, G., Schwob, E., Schiebel, E., et al. (2004). A versatile toolbox for PCR-based tagging of yeast genes: new fluorescent proteins, more markers and promoter substitution cassettes. Yeast 21, 947-962.

Jannot, G., Bajan, S., Giguère, N.J., Bouasker, S., Banville, I.H., Piquet, S., Hutvagner, G., and Simard, M.J. (2011). The ribosomal protein RACK1 is required for microRNA function in both C. elegans and humans. EMBO Rep 12, 581-586.

Jansen, J.M., Wanless, A.G., Seidel, C.W., and Weiss, E.L. (2009). Cbk1 regulation of the RNA-binding protein Ssd1 integrates cell fate with translational control. Curr Biol 19, 2114-2120.

Jansen, R.P., Dowzer, C., Michaelis, C., Galova, M., and Nasmyth, K. (1996). Mother cellspecific HO expression in budding yeast depends on the unconventional myosin myo4p and other cytoplasmic proteins. Cell 84, 687-697. 
Jeong, H.T., Oowatari, Y., Abe, M., Tanaka, K., Matsuda, H., and Kawamukai, M. (2004). Interaction between a negative regulator $(\mathrm{Msa} 2 / \mathrm{Nrd} 1)$ and a positive regulator $(\mathrm{Cpc} 2)$ of sexual differentiation in Schizosaccharomyces pombe. Biosci Biotechnol Biochem $68,1621-1626$.

Kadrmas, J.L., Smith, M.A., Pronovost, S.M., and Beckerle, M.C. (2007). Characterization of RACK1 function in Drosophila development. Dev Dyn 236, 2207-2215.

Kang, D., Gho, S., Suh, M., and Kang, C. (2002). Highly Sensitive and Fast Protein Detection with Coomassie Brilliant Blue in Sodium Dodecyl Sulfate-Polyacrylamide Gel Electrophoresis. Bull Korean Chem Soc 11, 1511-1512.

Kanshin, E., Michnick, S.W., and Thibault, P. (2013). Displacement of N/Q-rich peptides on $\mathrm{TiO}_{2}$ beads enhances the depth and coverage of yeast phosphoproteome analyses. $\mathrm{J}$ Proteome Res 12, 2905-2913.

Kiely, P.A., Baillie, G.S., Barrett, R., Buckley, D.A., Adams, D.R., Houslay, M.D., and O'Connor, R. (2009). Phosphorylation of RACK1 on tyrosine 52 by c-Abl is required for insulin-like growth factor I-mediated regulation of focal adhesion kinase. J Biol Chem 284, 20263-20274.

Kiely, P.A., Leahy, M., O'Gorman, D., and O'Connor, R. (2005). RACK1-mediated integration of adhesion and insulin-like growth factor I (IGF-I) signaling and cell migration are defective in cells expressing an IGF-I receptor mutated at tyrosines 1250 and 1251. J Biol Chem 280, 7624-7633.

Kiely, P.A., O'Gorman, D., Luong, K., Ron, D., and O'Connor, R. (2006). Insulin-like growth factor I controls a mutually exclusive association of RACK1 with protein phosphatase $2 \mathrm{~A}$ and $\beta 1$ integrin to promote cell migration. Mol Cell Biol 26, 4041-4051.

Kim, S.W., Joo, Y.J., and Kim, J. (2010). Asc1p, a ribosomal protein, plays a pivotal role in cellular adhesion and virulence in Candida albicans. J Microbiol 48, 842-848.

Kiss-László, Z., Henry, Y., Bachellerie, J.P., Caizergues-Ferrer, M., and Kiss, T. (1996). Sitespecific ribose methylation of preribosomal RNA: a novel function for small nucleolar RNAs. Cell 85, 1077-1088.

Kleinschmidt, M., Schulz, R., and Braus, G.H. (2006). The yeast CPC2/ASC1 gene is regulated by the transcription factors Fhl1p and Ifh1p. Curr Genet 49, 218-228.

Knaus, M., Cameroni, E., Pedruzzi, I., Tatchell, K., De Virgilio, C., and Peter, M. (2005). The Bud14p-Glc7p complex functions as a cortical regulator of dynein in budding yeast. EMBO J 24, 3000-3011.

Kong, Q., Wang, L., Liu, Z., Kwon, N.J., Kim, S.C., and Yu, J.H. (2013). Gß-like CpcB plays a crucial role for growth and development of Aspergillus nidulans and Aspergillus fumigatus. PLoS One 8, e70355.

Kouba, T., Rutkai, E., Karásková, M., and Valášek, L. (2012). The eIF3c/NIP1 PCI domain interacts with RNA and RACK1/ASC1 and promotes assembly of translation preinitiation complexes. Nucleic Acids Res 40, 2683-2699. 
Kuroha, K., Akamatsu, M., Dimitrova, L., Ito, T., Kato, Y., Shirahige, K., and Inada, T. (2010). Receptor for activated C kinase 1 stimulates nascent polypeptide-dependent translation arrest. EMBO Rep 11, 956-961.

Laemmli, U.K. (1970). Cleavage of structural proteins during the assembly of the head of bacteriophage T4. Nature 227, 680-685.

Langmead, B., and Salzberg, S.L. (2012). Fast gapped-read alignment with Bowtie 2. Nat Methods 9, 357-359.

Lee, C.D., and Tu, B.P. (2015). Glucose-Regulated Phosphorylation of the PUF Protein Puf3 Regulates the Translational Fate of Its Bound mRNAs and Association with RNA Granules. Cell Rep 11, 1638-1650.

Lenssen, E., James, N., Pedruzzi, I., Dubouloz, F., Cameroni, E., Bisig, R., Maillet, L., Werner, M., Roosen, J., Petrovic, K., et al. (2005). The Ccr4-Not complex independently controls both Msn2-dependent transcriptional activation--via a newly identified Glc7/Bud14 type I protein phosphatase module--and TFIID promoter distribution. Mol Cell Biol 25, 488-498.

Letzring, D.P., Dean, K.M., and Grayhack, E.J. (2010). Control of translation efficiency in yeast by codon-anticodon interactions. RNA 16, 2516-2528.

Letzring, D.P., Wolf, A.S., Brule, C.E., and Grayhack, E.J. (2013). Translation of CGA codon repeats in yeast involves quality control components and ribosomal protein L1. RNA 19, 1208-1217.

Li, D., and Roberts, R. (2001). WD-repeat proteins: structure characteristics, biological function, and their involvement in human diseases. Cell Mol Life Sci 58, 2085-2097.

Li, J.J., and Xie, D. (2015). RACK1, a versatile hub in cancer. Oncogene 34, 1890-1898.

Li, S., Esterberg, R., Lachance, V., Ren, D., Radde-Gallwitz, K., Chi, F., Parent, J.L., Fritz, A., and Chen, P. (2011). Rack1 is required for Vangl2 membrane localization and planar cell polarity signaling while attenuating canonical Wnt activity. Proc Natl Acad Sci U S A 108, 2264-2269.

Liebman, S.W., and Chernoff, Y.O. (2012). Prions in yeast. Genetics 191, 1041-1072.

Liedtke, C.M., Yun, C.H., Kyle, N., and Wang, D. (2002). Protein kinase C $\varepsilon$-dependent regulation of cystic fibrosis transmembrane regulator involves binding to a receptor for activated C kinase (RACK1) and RACK1 binding to $\mathrm{Na}^{+} / \mathrm{H}^{+}$exchange regulatory factor. J Biol Chem 277, 22925-22933.

Lin, J., Lee, D., Choi, Y., and Lee, S.Y. (2015). The scaffold protein RACK1 mediates the RANKL-dependent activation of p38 MAPK in osteoclast precursors. Sci Signal 8, ra54.

Lindstrom, D.L., Squazzo, S.L., Muster, N., Burckin, T.A., Wachter, K.C., Emigh, C.A., McCleery, J.A., Yates, J.R., 3rd, and Hartzog, G.A. (2003). Dual roles for Spt5 in premRNA processing and transcription elongation revealed by identification of Spt5associated proteins. Mol Cell Biol 23, 1368-1378. 
Link, A.J., Eng, J., Schieltz, D.M., Carmack, E., Mize, G.J., Morris, D.R., Garvik, B.M., and Yates, J.R., 3rd (1999). Direct analysis of protein complexes using mass spectrometry. Nat Biotechnol 17, 676-682.

Liu, L., Zeng, M., Hausladen, A., Heitman, J., and Stamler, J.S. (2000). Protection from nitrosative stress by yeast flavohemoglobin. Proc Natl Acad Sci U S A 97, 4672-4676.

Liu, X., Nie, X., Ding, Y., and Chen, J. (2010). Asc1, a WD-repeat protein, is required for hyphal development and virulence in Candida albicans. Acta Biochim Biophys Sin (Shanghai) 42, 793-800.

Liu, Y., Warfield, L., Zhang, C., Luo, J., Allen, J., Lang, W.H., Ranish, J., Shokat, K.M., and Hahn, S. (2009). Phosphorylation of the transcription elongation factor Spt5 by yeast Bur1 kinase stimulates recruitment of the PAF complex. Mol Cell Biol 29, 4852-4863.

Liu, Y.V., Baek, J.H., Zhang, H., Diez, R., Cole, R.N., and Semenza, G.L. (2007a). RACK1 competes with HSP90 for binding to HIF-1 $\alpha$ and is required for $\mathrm{O}_{2}$-independent and HSP90 inhibitor-induced degradation of HIF-1 $\alpha$. Mol Cell 25, 207-217.

Liu, Y.V., Hubbi, M.E., Pan, F., McDonald, K.R., Mansharamani, M., Cole, R.N., Liu, J.O., and Semenza, G.L. (2007b). Calcineurin promotes hypoxia-inducible factor $1 \alpha$ expression by dephosphorylating RACK1 and blocking RACK1 dimerization. J Biol Chem 282, 37064-37073.

Loewith, R., Jacinto, E., Wullschleger, S., Lorberg, A., Crespo, J.L., Bonenfant, D., Oppliger, W., Jenoe, P., and Hall, M.N. (2002). Two TOR complexes, only one of which is rapamycin sensitive, have distinct roles in cell growth control. Mol Cell 10, 457-468.

Lohse, M.B., Zordan, R.E., Cain, C.W., and Johnson, A.D. (2010). Distinct class of DNAbinding domains is exemplified by a master regulator of phenotypic switching in Candida albicans. Proc Natl Acad Sci U S A 107, 14105-14110.

López-Bergami, P., Habelhah, H., Bhoumik, A., Zhang, W., Wang, L.H., and Ronai, Z. (2005). RACK1 mediates activation of JNK by protein kinase C [corrected]. Mol Cell $19,309-320$.

Loreni, F., Iadevaia, V., Tino, E., Caldarola, S., and Amaldi, F. (2005). RACK1 mRNA translation is regulated via a rapamycin-sensitive pathway and coordinated with ribosomal protein synthesis. FEBS Lett 579, 5517-5520.

Lu, J., and Deutsch, C. (2008). Electrostatics in the ribosomal tunnel modulate chain elongation rates. J Mol Biol 384, 73-86.

Lu, K., Psakhye, I., and Jentsch, S. (2014). Autophagic clearance of polyQ proteins mediated by ubiquitin-Atg8 adaptors of the conserved CUET protein family. Cell 158, 549-563.

Majzoub, K., Hafirassou, M.L., Meignin, C., Goto, A., Marzi, S., Fedorova, A., Verdier, Y., Vinh, J., Hoffmann, J.A., Martin, F., et al. (2014). RACK1 controls IRES-mediated translation of viruses. Cell 159, 1086-1095.

Mamidipudi, V., Zhang, J., Lee, K.C., and Cartwright, C.A. (2004). RACK1 regulates G $1 / S$ progression by suppressing Src kinase activity. Mol Cell Biol 24, 6788-6798. 
Manogaran, A.L., Hong, J.Y., Hufana, J., Tyedmers, J., Lindquist, S., and Liebman, S.W. (2011). Prion formation and polyglutamine aggregation are controlled by two classes of genes. PLoS Genet 7, e1001386.

Mašek, T., Valášek, L., and Pospišek, M. (2011). Polysome analysis and RNA purification from sucrose gradients. Methods Mol Biol 703, 293-309.

Matsuda, R., Ikeuchi, K., Nomura, S., and Inada, T. (2014). Protein quality control systems associated with no-go and nonstop mRNA surveillance in yeast. Genes Cells 19, 1-12.

Mazanek, M., Mituloviae, G., Herzog, F., Stingl, C., Hutchins, J.R., Peters, J.M., and Mechtler, K. (2007). Titanium dioxide as a chemo-affinity solid phase in offline phosphopeptide chromatography prior to HPLC-MS/MS analysis. Nat Protoc 2, 10591069.

McLeod, M., Shor, B., Caporaso, A., Wang, W., Chen, H., and Hu, L. (2000). Cpc2, a fission yeast homologue of mammalian RACK1 protein, interacts with Ran1 (Pat1) kinase To regulate cell cycle progression and meiotic development. Mol Cell Biol 20, 40164027.

Melamed, D., Bar-Ziv, L., Truzman, Y., and Arava, Y. (2010). Asc1 supports cell-wall integrity near bud sites by a Pkc1 independent mechanism. PLoS One 5, e11389.

Michelot, A., Grassart, A., Okreglak, V., Costanzo, M., Boone, C., and Drubin, D.G. (2013). Actin filament elongation in Arp2/3-derived networks is controlled by three distinct mechanisms. Dev Cell 24, 182-195.

Mizukami, J., Takaesu, G., Akatsuka, H., Sakurai, H., Ninomiya-Tsuji, J., Matsumoto, K., and Sakurai, N. (2002). Receptor activator of NF- $\kappa B$ ligand (RANKL) activates TAK1 mitogen-activated protein kinase kinase kinase through a signaling complex containing RANK, TAB2, and TRAF6. Mol Cell Biol 22, 992-1000.

Mok, J., Kim, P.M., Lam, H.Y., Piccirillo, S., Zhou, X., Jeschke, G.R., Sheridan, D.L., Parker, S.A., Desai, V., Jwa, M., et al. (2011). Deciphering protein kinase specificity through large-scale analysis of yeast phosphorylation site motifs. Sci Signal 3, ra12.

Müller, M., Kötter, P., Behrendt, C., Walter, E., Scheckhuber, C.Q., Entian, K.D., and Reichert, A.S. (2015). Synthetic quantitative array technology identifies the Ubp3Bre5 deubiquitinase complex as a negative regulator of mitophagy. Cell Rep 10, 12151225 .

Mumberg, D., Müller, R., and Funk, M. (1994). Regulatable promoters of Saccharomyces cerevisiae: comparison of transcriptional activity and their use for heterologous expression. Nucleic Acids Res 22, 5767-5768.

Murzin, A.G. (1992). Structural principles for the propeller assembly of $\beta$-sheets: the preference for seven-fold symmetry. Proteins 14, 191-201.

Musti, A.M., Treier, M., and Bohmann, D. (1997). Reduced ubiquitin-dependent degradation of c-Jun after phosphorylation by MAP kinases. Science 275, 400-402. 
Nakashima, A., Chen, L., Thao, N.P., Fujiwara, M., Wong, H.L., Kuwano, M., Umemura, K., Shirasu, K., Kawasaki, T., and Shimamoto, K. (2008). RACK1 functions in rice innate immunity by interacting with the Rac1 immune complex. Plant Cell 20, 2265-2279.

Neasta, J., Kiely, P.A., He, D.Y., Adams, D.R., O'Connor, R., and Ron, D. (2012). Direct interaction between scaffolding proteins RACK1 and 14-3-3 $\zeta$ regulates brainderived neurotrophic factor (BDNF) transcription. J Biol Chem 287, 322-336.

Ni, J., Tien, A.L., and Fournier, M.J. (1997). Small nucleolar RNAs direct site-specific synthesis of pseudouridine in ribosomal RNA. Cell 89, 565-573.

Nilsson, J., Sengupta, J., Frank, J., and Nissen, P. (2004). Regulation of eukaryotic translation by the RACK1 protein: a platform for signalling molecules on the ribosome. EMBO Rep 5, 1137-1141.

Nuñez, A., Franco, A., Madrid, M., Soto, T., Vicente, J., Gacto, M., and Cansado, J. (2009). Role for RACK1 orthologue Cpc2 in the modulation of stress response in fission yeast. Mol Biol Cell 20, 3996-4009.

Nuñez, A., Franco, A., Soto, T., Vicente, J., Gacto, M., and Cansado, J. (2010). Fission yeast receptor of activated $\mathrm{C}$ kinase (RACK1) ortholog $\mathrm{Cpc} 2$ regulates mitotic commitment through Wee1 kinase. J Biol Chem 285, 41366-41373.

Ogur, M., St. John, R., and Nagai, S. (1957). Tetrazolium overlay technique for population studies of respiration deficiency in yeast. Science 125, 928-929.

Olivas, W., and Parker, R. (2000). The Puf3 protein is a transcript-specific regulator of mRNA degradation in yeast. EMBO J 19, 6602-6611.

Olsen, J.V., Blagoev, B., Gnad, F., Macek, B., Kumar, C., Mortensen, P., and Mann, M. (2006). Global, in vivo, and site-specific phosphorylation dynamics in signaling networks. Cell 127, 635-648.

Olsen, J.V., and Mann, M. (2004). Improved peptide identification in proteomics by two consecutive stages of mass spectrometric fragmentation. Proc Natl Acad Sci U S A $101,13417-13422$.

Otsuka, M., Takata, A., Yoshikawa, T., Kojima, K., Kishikawa, T., Shibata, C., Takekawa, M., Yoshida, H., Omata, M., and Koike, K. (2011). Receptor for activated protein kinase $\mathrm{C}$ : requirement for efficient microRNA function and reduced expression in hepatocellular carcinoma. PLoS One 6, e24359.

Palmer, D.A., Thompson, J.K., Li, L., Prat, A., and Wang, P. (2006). Gib2, a novel G $\beta$ like/RACK1 homolog, functions as a G $\beta$ subunit in cAMP signaling and is essential in Cryptococcus neoformans. J Biol Chem 281, 32596-32605.

Papavassiliou, A.G., Treier, M., and Bohmann, D. (1995). Intramolecular signal transduction in c-Jun. EMBO J 14, 2014-2019.

Paquin, N., Ménade, M., Poirier, G., Donato, D., Drouet, E., and Chartrand, P. (2007). Local activation of yeast $A S H 1$ mRNA translation through phosphorylation of Khd1p by the casein kinase Yck1p. Mol Cell 26, 795-809. 
Parsons, A.B., Brost, R.L., Ding, H., Li, Z., Zhang, C., Sheikh, B., Brown, G.W., Kane, P.M., Hughes, T.R., and Boone, C. (2004). Integration of chemical-genetic and genetic interaction data links bioactive compounds to cellular target pathways. Nat Biotechnol $22,62-69$.

Qu, L.H., Henry, Y., Nicoloso, M., Michot, B., Azum, M.C., Renalier, M.H., CaizerguesFerrer, M., and Bachellerie, J.P. (1995). U24, a novel intron-encoded small nucleolar RNA with two $12 \mathrm{nt}$ long, phylogenetically conserved complementarities to $28 \mathrm{~S}$ rRNA. Nucleic Acids Res 23, 2669-2676.

Rabl, J., Leibundgut, M., Ataide, S.F., Haag, A., and Ban, N. (2011). Crystal structure of the eukaryotic 40S ribosomal subunit in complex with initiation factor 1. Science 331, 730-736.

Rachfall, N., Schmitt, K., Bandau, S., Smolinski, N., Ehrenreich, A., Valerius, O., and Braus, G.H. (2013). RACK1/Asc1p, a ribosomal node in cellular signaling. Mol Cell Proteomics 12, 87-105.

Radi, R. (2004). Nitric oxide, oxidants, and protein tyrosine nitration. Proc Natl Acad Sci U S A $101,4003-4008$.

Rappsilber, J., Ishihama, Y., and Mann, M. (2003). Stop and go extraction tips for matrixassisted laser desorption/ionization, nanoelectrospray, and LC/MS sample pretreatment in proteomics. Anal Chem 75, 663-670.

Rappsilber, J., Mann, M., and Ishihama, Y. (2007). Protocol for micro-purification, enrichment, pre-fractionation and storage of peptides for proteomics using StageTips. Nat Protoc 2, 1896-1906.

Regmi, S., Rothberg, K.G., Hubbard, J.G., and Ruben, L. (2008). The RACK1 signal anchor protein from Trypanosoma brucei associates with eukaryotic elongation factor 1A: a role for translational control in cytokinesis. Mol Microbiol 70, 724-745.

Rivero-Gutiérrez, B., Anzola, A., Martínez-Augustin, O., and de Medina, F.S. (2014). Stainfree detection as loading control alternative to Ponceau and housekeeping protein immunodetection in Western blotting. Anal Biochem 467, 1-3.

Robles, M.S., Boyault, C., Knutti, D., Padmanabhan, K., and Weitz, C.J. (2010). Identification of RACK1 and protein kinase $\mathrm{C} \alpha$ as integral components of the mammalian circadian clock. Science 327, 463-466.

Ron, D., Chen, C.H., Caldwell, J., Jamieson, L., Orr, E., and Mochly-Rosen, D. (1994). Cloning of an intracellular receptor for protein kinase $C$ : a homolog of the $\beta$ subunit of G proteins. Proc Natl Acad Sci U S A 91, 839-843.

Ron, D., Jiang, Z., Yao, L., Vagts, A., Diamond, I., and Gordon, A. (1999). Coordinated movement of RACK1 with activated $\beta$ IIPKC. J Biol Chem 274, 27039-27046.

Ron, D., Luo, J., and Mochly-Rosen, D. (1995). C2 region-derived peptides inhibit translocation and function of $\beta$ protein kinase $\mathrm{C}$ in vivo. J Biol Chem 270, 2418024187. 
Ron, D., and Mochly-Rosen, D. (1994). Agonists and antagonists of protein kinase C function, derived from its binding proteins. J Biol Chem 269, 21395-21398.

Rotenberg, M.O., Moritz, M., and Woolford, J.L., Jr. (1988). Depletion of Saccharomyces cerevisiae ribosomal protein L16 causes a decrease in 60S ribosomal subunits and formation of half-mer polyribosomes. Genes Dev 2, 160-172.

Ruan, Y., Sun, L., Hao, Y., Wang, L., Xu, J., Zhang, W., Xie, J., Guo, L., Zhou, L., Yun, X., et al. (2012). Ribosomal RACK1 promotes chemoresistance and growth in human hepatocellular carcinoma. J Clin Invest 122, 2554-2566.

Rubinfeld, H., and Seger, R. (2005). The ERK cascade: a prototype of MAPK signaling. Mol Biotechnol 31, 151-174.

Ruiz Carrillo, D., Chandrasekaran, R., Nilsson, M., Cornvik, T., Liew, C.W., Tan, S.M., and Lescar, J. (2012). Structure of human Rack1 protein at a resolution of 2.45 A. Acta Crystallogr Sect F Struct Biol Cryst Commun 68, 867-872.

Saiki, R.K., Scharf, S., Faloona, F., Mullis, K.B., Horn, G.T., Erlich, H.A., and Arnheim, N. (1985). Enzymatic amplification of $\beta$-globin genomic sequences and restriction site analysis for diagnosis of sickle cell anemia. Science 230, 1350-1354.

Saint-Georges, Y., Garcia, M., Delaveau, T., Jourdren, L., Le Crom, S., Lemoine, S., Tanty, V., Devaux, F., and Jacq, C. (2008). Yeast mitochondrial biogenesis: a role for the PUF RNA-binding protein Puf3p in mRNA localization. PLoS One 3, e2293.

Satoh, R., Morita, T., Takada, H., Kita, A., Ishiwata, S., Doi, A., Hagihara, K., Taga, A., Matsumura, Y., Tohda, H., et al. (2009). Role of the RNA-binding protein Nrd1 and Pmk1 mitogen-activated protein kinase in the regulation of myosin mRNA stability in fission yeast. Mol Biol Cell 20, 2473-2485.

Schneider, D.A., French, S.L., Osheim, Y.N., Bailey, A.O., Vu, L., Dodd, J., Yates, J.R., Beyer, A.L., and Nomura, M. (2006). RNA polymerase II elongation factors Spt4p and Spt5p play roles in transcription elongation by RNA polymerase I and rRNA processing. Proc Natl Acad Sci U S A 103, 12707-12712.

Schwartz, D., and Gygi, S.P. (2005). An iterative statistical approach to the identification of protein phosphorylation motifs from large-scale data sets. Nat Biotechnol 23, 13911398.

Semenza, G.L. (2013). HIF-1 mediates metabolic responses to intratumoral hypoxia and oncogenic mutations. J Clin Invest 123, 3664-3671.

Semenza, G.L., Roth, P.H., Fang, H.M., and Wang, G.L. (1994). Transcriptional regulation of genes encoding glycolytic enzymes by hypoxia-inducible factor 1. J Biol Chem 269, 23757-23763.

Sengupta, J., Nilsson, J., Gursky, R., Spahn, C.M., Nissen, P., and Frank, J. (2004). Identification of the versatile scaffold protein RACK1 on the eukaryotic ribosome by cryo-EM. Nat Struct Mol Biol 11, 957-962. 
Sezen, B., Seedorf, M., and Schiebel, E. (2009). The SESA network links duplication of the yeast centrosome with the protein translation machinery. Genes Dev 23, 1559-1570.

Sha, Z., Brill, L.M., Cabrera, R., Kleifeld, O., Scheliga, J.S., Glickman, M.H., Chang, E.C., and Wolf, D.A. (2009). The eIF3 interactome reveals the translasome, a supercomplex linking protein synthesis and degradation machineries. Mol Cell 36, 141-152.

Shen, Z., St-Denis, A., and Chartrand, P. (2010). Cotranscriptional recruitment of She2p by RNA pol II elongation factor Spt4-Spt5/DSIF promotes mRNA localization to the yeast bud. Genes Dev 24, 1914-1926.

Shevchenko, A., Wilm, M., Vorm, O., and Mann, M. (1996). Mass spectrometric sequencing of proteins silver-stained polyacrylamide gels. Anal Chem 68, 850-858.

Shih, S.C., Prag, G., Francis, S.A., Sutanto, M.A., Hurley, J.H., and Hicke, L. (2003). A ubiquitin-binding motif required for intramolecular monoubiquitylation, the CUE domain. EMBO J 22, 1273-1281.

Sil, A., and Herskowitz, I. (1996). Identification of asymmetrically localized determinant, Ash1p, required for lineage-specific transcription of the yeast $H O$ gene. Cell 84, 711722.

Smolka, M.B., Albuquerque, C.P., Chen, S.H., and Zhou, H. (2007). Proteome-wide identification of in vivo targets of DNA damage checkpoint kinases. Proc Natl Acad Sci U S A 104, 10364-10369.

Southern, E.M. (1975). Detection of specific sequences among DNA fragments separated by gel electrophoresis. J Mol Biol 98, 503-517.

Speth, C., Willing, E.M., Rausch, S., Schneeberger, K., and Laubinger, S. (2013). RACK1 scaffold proteins influence miRNA abundance in Arabidopsis. Plant J 76, 433-445.

Stark, C., Breitkreutz, B.J., Reguly, T., Boucher, L., Breitkreutz, A., and Tyers, M. (2006). BioGRID: a general repository for interaction datasets. Nucleic Acids Res 34, D535539.

Stirnimann, C.U., Petsalaki, E., Russell, R.B., and Müller, C.W. (2010). WD40 proteins propel cellular networks. Trends Biochem Sci 35, 565-574.

Subauste, M.C., Ventura-Holman, T., Du, L., Subauste, J.S., Chan, S.L., Yu, V.C., and Maher, J.F. (2009). RACK1 downregulates levels of the pro-apoptotic protein Fem1b in apoptosis-resistant colon cancer cells. Cancer Biol Ther 8, 2297-2305.

Swaney, D.L., Beltrao, P., Starita, L., Guo, A., Rush, J., Fields, S., Krogan, N.J., and Villén, J. (2013). Global analysis of phosphorylation and ubiquitylation cross-talk in protein degradation. Nat Methods 10, 676-682.

Tarnowski, K., Fituch, K., Szczepanowski, R.H., Dadlez, M., and Kaus-Drobek, M. (2014). Patterns of structural dynamics in RACK1 protein retained throughout evolution: a hydrogen-deuterium exchange study of three orthologs. Protein Sci 23, 639-651. 
Tarumoto, Y., Kanoh, J., and Ishikawa, F. (2013). Receptor for activated C-kinase (RACK1) homolog Cpc2 facilitates the general amino acid control response through Gcn2 kinase in fission yeast. J Biol Chem 288, 19260-19268.

Tedford, K., Kim, S., Sa, D., Stevens, K., and Tyers, M. (1997). Regulation of the mating pheromone and invasive growth responses in yeast by two MAP kinase substrates. Curr Biol 7, 228-238.

Thornton, C., Tang, K.C., Phamluong, K., Luong, K., Vagts, A., Nikanjam, D., Yaka, R., and Ron, D. (2004). Spatial and temporal regulation of RACK1 function and N-methyl-Daspartate receptor activity through WD40 motif-mediated dimerization. J Biol Chem $279,31357-31364$.

Tkach, J.M., Yimit, A., Lee, A.Y., Riffle, M., Costanzo, M., Jaschob, D., Hendry, J.A., Ou, J., Moffat, J., Boone, C., et al. (2012). Dissecting DNA damage response pathways by analysing protein localization and abundance changes during DNA replication stress. Nat Cell Biol 14, 966-976.

Tsukahara, K., Yamamoto, H., and Okayama, H. (1998). An RNA binding protein negatively controlling differentiation in fission yeast. Mol Cell Biol 18, 4488-4498.

Ullah, H., Scappini, E.L., Moon, A.F., Williams, L.V., Armstrong, D.L., and Pedersen, L.C. (2008). Structure of a signal transduction regulator, RACK1, from Arabidopsis thaliana. Protein Sci 17, 1771-1780.

Urano, D., Czarnecki, O., Wang, X., Jones, A.M., and Chen, J.G. (2015). Arabidopsis receptor of activated $\mathrm{C}$ kinase1 phosphorylation by WITH NO LYSINE8 KINASE. Plant Physiol 167, 507-516.

Urban, J., Soulard, A., Huber, A., Lippman, S., Mukhopadhyay, D., Deloche, O., Wanke, V., Anrather, D., Ammerer, G., Riezman, H., et al. (2007). Sch9 is a major target of TORC1 in Saccharomyces cerevisiae. Mol Cell 26, 663-674.

Valerius, O., Kleinschmidt, M., Rachfall, N., Schulze, F., López Marín, S., Hoppert, M., Streckfuss-Bömeke, K., Fischer, C., and Braus, G.H. (2007). The Saccharomyces homolog of mammalian RACK1, Cpc2/Asc1p, is required for FLO11-dependent adhesive growth and dimorphism. Mol Cell Proteomics 6, 1968-1979.

Vani, K., Yang, G., and Mohler, J. (1997). Isolation and cloning of a Drosophila homolog to the mammalian RACK1 gene, implicated in PKC-mediated signalling. Biochim Biophys Acta 1358, 67-71.

Volta, V., Beugnet, A., Gallo, S., Magri, L., Brina, D., Pesce, E., Calamita, P., Sanvito, F., and Biffo, S. (2012). RACK1 depletion in a mouse model causes lethality, pigmentation deficits and reduction in protein synthesis efficiency. Cell Mol Life Sci $70,1439-1450$.

Vomastek, T., Iwanicki, M.P., Schaeffer, H.J., Tarcsafalvi, A., Parsons, J.T., and Weber, M.J. (2007). RACK1 targets the extracellular signal-regulated kinase/mitogen-activated protein kinase pathway to link integrin engagement with focal adhesion disassembly and cell motility. Mol Cell Biol 27, 8296-8305. 
Voytas, D. (2001). Agarose gel electrophoresis. Curr Protoc Mol Biol Chapter 2, Unit2 5A.

Wang, G.L., and Semenza, G.L. (1995). Purification and characterization of hypoxiainducible factor 1. J Biol Chem 270, 1230-1237.

Wang, L., Berndt, P., Xia, X., Kahnt, J., and Kahmann, R. (2011). A seven-WD40 protein related to human RACK1 regulates mating and virulence in Ustilago maydis. Mol Microbiol 81, 1484-1498.

Wang, M., Herrmann, C.J., Simonovic, M., Szklarczyk, D., and von Mering, C. (2015a). Version 4.0 of PaxDb: Protein abundance data, integrated across model organisms, tissues, and cell-lines. Proteomics 15, 3163-3168.

Wang, M., Weiss, M., Simonovic, M., Haertinger, G., Schrimpf, S.P., Hengartner, M.O., and von Mering, C. (2012). PaxDb, a database of protein abundance averages across all three domains of life. Mol Cell Proteomics 11, 492-500.

Wang, Q., Zhou, S., Wang, J.Y., Cao, J., Zhang, X., Wang, J., Han, K., Cheng, Q., Qiu, G., Zhao, Y., et al. (2015b). RACK1 antagonizes TNF- $\alpha$-induced cell death by promoting p38 activation. Sci Rep 5, 14298.

Wang, S., Sakai, H., and Wiedmann, M. (1995). NAC covers ribosome-associated nascent chains thereby forming a protective environment for regions of nascent chains just emerging from the peptidyl transferase center. J Cell Biol 130, 519-528.

Wang, X., and Huang, L. (2008). Identifying dynamic interactors of protein complexes by quantitative mass spectrometry. Mol Cell Proteomics 7, 46-57.

Wang, Y., Shen, G., Gong, J., Shen, D., Whittington, A., Qing, J., Treloar, J., Boisvert, S., Zhang, Z., Yang, C., et al. (2014). Noncanonical G $\beta$ Gib2 is a scaffolding protein promoting cAMP signaling through functions of Ras1 and Cac1 proteins in Cryptococcus neoformans. J Biol Chem 289, 12202-12216.

Wang, Z., Zhang, B., Jiang, L., Zeng, X., Chen, Y., Feng, X., Guo, Y., and Chen, Q. (2009). RACK1, an excellent predictor for poor clinical outcome in oral squamous carcinoma, similar to Ki67. Eur J Cancer 45, 490-496.

Warner, J.R., and McIntosh, K.B. (2009). How common are extraribosomal functions of ribosomal proteins? Mol Cell 34, 3-11.

Watkins, N.J., and Bohnsack, M.T. (2012). The box C/D and H/ACA snoRNPs: key players in the modification, processing and the dynamic folding of ribosomal RNA. Wiley Interdiscip Rev RNA 3, 397-414.

Wehner, P., Shnitsar, I., Urlaub, H., and Borchers, A. (2011). RACK1 is a novel interaction partner of PTK7 that is required for neural tube closure. Development 138, 1321-1327.

Weinert, B.T., Schölz, C., Wagner, S.A., Iesmantavicius, V., Su, D., Daniel, J.A., and Choudhary, C. (2013). Lysine succinylation is a frequently occurring modification in prokaryotes and eukaryotes and extensively overlaps with acetylation. Cell Rep 4, 842-851. 
Weiss, C., Schneider, S., Wagner, E.F., Zhang, X., Seto, E., and Bohmann, D. (2003). JNK phosphorylation relieves HDAC3-dependent suppression of the transcriptional activity of c-Jun. EMBO J 22, 3686-3695.

Wek, S.A., Zhu, S., and Wek, R.C. (1995). The histidyl-tRNA synthetase-related sequence in the eIF- $2 \alpha$ protein kinase GCN2 interacts with tRNA and is required for activation in response to starvation for different amino acids. Mol Cell Biol 15, 4497-4506.

Wen, Y., and Shatkin, A.J. (1999). Transcription elongation factor hSPT5 stimulates mRNA capping. Genes Dev 13, 1774-1779.

Wessel, D., and Flügge, U.I. (1984). A method for the quantitative recovery of protein in dilute solution in the presence of detergents and lipids. Anal Biochem 138, 141-143.

Wilson-Grady, J.T., Villén, J., and Gygi, S.P. (2008). Phosphoproteome analysis of fission yeast. J Proteome Res 7, 1088-1097.

Wilson, M.D., Harreman, M., Taschner, M., Reid, J., Walker, J., Erdjument-Bromage, H., Tempst, P., and Svejstrup, J.Q. (2013). Proteasome-mediated processing of Def1, a critical step in the cellular response to transcription stress. Cell 154, 983-995.

Wiśniewski, J.R., Zougman, A., Nagaraj, N., and Mann, M. (2009). Universal sample preparation method for proteome analysis. Nat Methods 6, 359-362.

Wolf, A.S., and Grayhack, E.J. (2015). Asc1, homolog of human RACK1, prevents frameshifting in yeast by ribosomes stalled at CGA codon repeats. RNA 21, 935-945.

Woodcock, D.M., Crowther, P.J., Doherty, J., Jefferson, S., DeCruz, E., Noyer-Weidner, M., Smith, S.S., Michael, M.Z., and Graham, M.W. (1989). Quantitative evaluation of Escherichia coli host strains for tolerance to cytosine methylation in plasmid and phage recombinants. Nucleic Acids Res 17, 3469-3478.

Wu, C., Lytvyn, V., Thomas, D.Y., and Leberer, E. (1997). The phosphorylation site for Ste20p-like protein kinases is essential for the function of myosin-I in yeast. J Biol Chem 272, 30623-30626.

Yaka, R., He, D.Y., Phamluong, K., and Ron, D. (2003). Pituitary adenylate cyclaseactivating polypeptide (PACAP(1-38)) enhances N-methyl-D-aspartate receptor function and brain-derived neurotrophic factor expression via RACK1. J Biol Chem 278, 9630-9638.

Yaka, R., Thornton, C., Vagts, A.J., Phamluong, K., Bonci, A., and Ron, D. (2002). NMDA receptor function is regulated by the inhibitory scaffolding protein, RACK1. Proc Natl Acad Sci U S A 99, 5710-5715.

Yao, S., Neiman, A., and Prelich, G. (2000). BUR1 and BUR2 encode a divergent cyclindependent kinase-cyclin complex important for transcription in vivo. Mol Cell Biol 20, 7080-7087.

Yarwood, S.J., Steele, M.R., Scotland, G., Houslay, M.D., and Bolger, G.B. (1999). The RACK1 signaling scaffold protein selectively interacts with the cAMP-specific phosphodiesterase PDE4D5 isoform. J Biol Chem 274, 14909-14917. 
Yatime, L., Hein, K.L., Nilsson, J., and Nissen, P. (2011). Structure of the RACK1 dimer from Saccharomyces cerevisiae. J Mol Biol 411, 486-498.

Zeller, C.E., Parnell, S.C., and Dohlman, H.G. (2007). The RACK1 ortholog Asc1 functions as a G-protein $\beta$ subunit coupled to glucose responsiveness in yeast. J Biol Chem $282,25168-25176$.

Zhang, D., Chen, L., Li, D., Lv, B., Chen, Y., Chen, J., XuejiaoYan, and Liang, J. (2014). OsRACK1 is involved in abscisic acid- and $\mathrm{H}_{2} \mathrm{O}_{2}$-mediated signaling to regulate seed germination in rice (Oryza sativa, L.). PLoS One 9, e97120.

Zhang, J., Zhu, F., Li, X., Dong, Z., Xu, Y., Peng, C., Li, S., Cho, Y.Y., Yao, K., Zykova, T.A., et al. (2012a). Rack1 protects N-terminal phosphorylated c-Jun from Fbw7mediated degradation. Oncogene 31, 1835-1844.

Zhang, W., Cheng, G.Z., Gong, J., Hermanto, U., Zong, C.S., Chan, J., Cheng, J.Q., and Wang, L.H. (2008). RACK1 and CIS mediate the degradation of BimEL in cancer cells. J Biol Chem 283, 16416-16426.

Zhang, W., Zong, C.S., Hermanto, U., Lopez-Bergami, P., Ronai, Z., and Wang, L.H. (2006). RACK1 recruits STAT3 specifically to insulin and insulin-like growth factor 1 receptors for activation, which is important for regulating anchorage-independent growth. Mol Cell Biol 26, 413-424.

Zhang, Y., Berndt, U., Gölz, H., Tais, A., Oellerer, S., Wölfle, T., Fitzke, E., and Rospert, S. (2012b). NAC functions as a modulator of SRP during the early steps of protein targeting to the endoplasmic reticulum. Mol Biol Cell 23, 3027-3040.

Zhao, Y., Wang, Q., Qiu, G., Zhou, S., Jing, Z., Wang, J., Wang, W., Cao, J., Han, K., Cheng, Q., et al. (2015). RACK1 Promotes Autophagy by Enhancing the Atg14L-Beclin 1Vps34-Vps15 Complex Formation upon Phosphorylation by AMPK. Cell Rep 13, 1407-1417.

Zhou, K., Kuo, W.H., Fillingham, J., and Greenblatt, J.F. (2009). Control of transcriptional elongation and cotranscriptional histone modification by the yeast BUR kinase substrate Spt5. Proc Natl Acad Sci U S A 106, 6956-6961. 


\section{Online tools and databases}

http://motif-x.med.harvard.edu

http://smart.embl-heidelberg.de

http://www.ch.embnet.org/software/BOX_form.html

http://www.genome.jp/kegg

http://www.pax-db.org

http://www.phosphogrid.org

http://www.rcsb.org

http://www.uniprot.org

http://www.yeastgenome.org 


\section{Supplementary Material}

\begin{tabular}{|c|c|c|c|c|c|}
\hline & + & 11 & 21 & 31 & 41 \\
\hline ASC1 SNR24 & ATGGCATCTA & ACGAAGTTTT & AGTTTTGAGA & GGTACCTTGG & AAGGTCACAA \\
\hline $\mathrm{sclp}$ & $\begin{array}{llll}M & A & S & N\end{array}$ & $\begin{array}{lll}E & \mathrm{~V} & \mathrm{~L}\end{array}$ & V L $\quad$ L & $\mathrm{L} \quad \mathrm{E}$ & $\mathrm{N}$ \\
\hline $\operatorname{ascl}^{-}$ & ATGGCATCTA & ACGAAGTTTT & AGTTTTGAGA & GGTACCTTGG & AAGGTCACAA \\
\hline & $\begin{array}{llll}M & A & S & N\end{array}$ & $\mathrm{E}$ & V L & G $\quad \mathrm{T} \quad \mathrm{L}$ & G \\
\hline & 51 & 61 & 71 & 81 & 91 \\
\hline$S C 1 \quad S N R 24$ & CGGTTGGGTC & AсAтCTTTGG & СТАСТTCTGC & TGGTCAACCA & AACCTATTGT \\
\hline $\mathrm{p}$ & $\begin{array}{lll}G & W & V\end{array}$ & $\begin{array}{llll} & \mathrm{S} & \mathrm{L} & \mathrm{A}\end{array}$ & $\mathrm{T} \quad \mathrm{S} \quad \mathrm{A}$ & $G \quad Q \quad P$ & $\begin{array}{llll}N & \mathrm{~L} & \mathrm{~L} & \mathrm{~L}\end{array}$ \\
\hline & CGGTTGGGTC & ACATCTTTGG & СТАСТTCTGC & TGGTCAACCA & АACСТАTTGT \\
\hline & $\begin{array}{lll}G & W & \mathrm{~V}\end{array}$ & $\begin{array}{llll}T & S & \mathrm{~L} & \mathrm{~A}\end{array}$ & $\mathrm{~T} \quad \mathrm{~S} \quad \mathrm{~A}$ & $\begin{array}{lll}G & Q & P\end{array}$ & $\begin{array}{llll}N & L & L & L\end{array}$ \\
\hline & 101 & 111 & 121 & 131 & 141 \\
\hline$S C 1 S N H$ & TGTCCGCTTC & CCGTGATAAG & АCTTTGATCT & CCTGGAAGTT & GACTGGTGAC \\
\hline & $S \quad A \quad S$ & $R \quad D \quad K$ & $\begin{array}{llll}T & \mathrm{~L} & \mathrm{I} & \mathrm{S}\end{array}$ & $\begin{array}{lll}W & K & L\end{array}$ & $\mathrm{~T} \quad \mathrm{G} \quad \mathrm{D}$ \\
\hline & TGTCCGCTTC & CCGTGATAAG & ACTTTGATCT & CCTGGAAGTT & GACTGGTGAC \\
\hline & $S \quad A \quad S$ & R $\quad \mathrm{D} \quad \mathrm{K}$ & $\begin{array}{llll}T & \mathrm{~L} & \mathrm{I} & \mathrm{S}\end{array}$ & W $\quad \mathrm{K} \quad \mathrm{L}$ & T G D \\
\hline & 51 & 1 & 171 & 181 & 191 \\
\hline 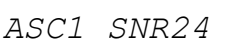 & GACCAAAAGT & TTGG------ & ---------- & --- & ---1 \\
\hline & $\begin{array}{llll}D & Q & K & F\end{array}$ & G & - & - & - \\
\hline & GACCAAAAGT & TTGGAGCAGC & TGAAGCTTCG & TACGCTGCAG & GTCGACAACC \\
\hline & $\begin{array}{llll}D & Q & K & F\end{array}$ & G A A & E A S & $\begin{array}{llll} & A & A & G\end{array}$ & $\begin{array}{lll}R & Q & P\end{array}$ \\
\hline & 201 & 211 & 221 & 231 & 241 \\
\hline $\mathrm{p}^{S}$ & $\begin{array}{c}---- \\
-\end{array}$ & - & - & - & $\begin{array}{l}----- \\
-\quad-\end{array}$ \\
\hline & СТTAАТАТАA & CTTCGTATAA & TGTATGCTAT & ACGAAGTTAT & TAGGTGATAT \\
\hline & $\mathrm{L} \quad \mathrm{I} \quad$ * & $\begin{array}{llllllllll} & - & - & -\end{array}$ & $-\quad-$ & $-\quad-$ & $\begin{array}{lllllllllllll} & - & - & -\end{array}$ \\
\hline & 251 & 261 & 271 & 281 & 291 \\
\hline SNI & ---- & ------ & $--\mathrm{TGTCCCAG}$ & TTAGATCTTT & CAAGGGTCAC \\
\hline & $-\quad-$ & - & $\begin{array}{llll}- & \mathrm{V} & \mathrm{P} & \mathrm{V}\end{array}$ & R $\quad \mathrm{S} \quad \mathrm{F}$ & $\begin{array}{lll}K & \mathrm{G} & \mathrm{H}\end{array}$ \\
\hline & AGATCCACT & AGTGGCCTAT & GCTGTCCCAG & TTAGATCTTT & CAAGGGTCAC \\
\hline & $-\quad-\quad-$ & - & $\begin{array}{llll}- & - & - & -\end{array}$ & - & - \\
\hline
\end{tabular}

Supplementary Figure 1. DNA and amino acid sequence alignments of the ASC1 SNR24 wildtype strain and the asc1 ${ }^{-}$strain.

The figure illustrates the differences in the DNA and amino acid sequence between the ASC1 SNR24 wild-type and the $\mathrm{ascl}^{-}$strain. Due to the presence of a stop codon (TAA labeled with an asterisks) within the loxP site in the asc $\mathrm{I}^{-}$strain, translation of the corresponding mRNA is abrogated leading to the formation of truncated Asc1p: 
Supplementary Table 1. LC-MS-based identification of Asc1p-derived phospho-peptides.

Phospho-sites with the highest post-translational modification (PTM) score are printed in black, and all other putative phospho-sites within the peptides in gray.

\begin{tabular}{|c|c|c|c|c|c|c|}
\hline $\begin{array}{l}\text { Amino } \\
\text { acid }\end{array}$ & Position & \begin{tabular}{|l|} 
Highest \\
PTM \\
score
\end{tabular} & Peptide sequence & $\begin{array}{l}\text { Spectral } \\
\text { counts }\end{array}$ & $\begin{array}{l}\text { Highest } \\
\text { Xcorr } \\
\text { (SequestHT) }\end{array}$ & $\begin{array}{l}\text { Highest } \\
\text { IonScore } \\
\text { (Mascot) }\end{array}$ \\
\hline $\mathrm{T}$ & 12 & 100.00 & \multirow{7}{*}{$\begin{array}{l}\text { GTLEGHNGWVTSLATSAGQPN } \\
\text { LLLSASR }\end{array}$} & \multirow{7}{*}{19} & \multirow{7}{*}{5.42} & \multirow{7}{*}{70} \\
\hline $\mathrm{T}$ & 21 & 45.5 & & & & \\
\hline$S$ & 22 & 33.3 & & & & \\
\hline$T$ & 25 & 60.1 & & & & \\
\hline$S$ & 26 & 39 & & & & \\
\hline$S$ & 35 & 9.9 & & & & \\
\hline$S$ & 37 & 79.6 & & & & \\
\hline$S$ & 120 & 100 & \multirow{3}{*}{ KAㅗMIISGSR } & \multirow{3}{*}{2} & \multirow{3}{*}{ - } & \multirow{3}{*}{47} \\
\hline$S$ & 124 & 0 & & & & \\
\hline$S$ & 126 & 0 & & & & \\
\hline$T$ & 143 & 100 & \multirow{2}{*}{ GQCLA寽LLGHNDWVSQVR } & \multirow{2}{*}{29} & \multirow{2}{*}{4.54} & \multirow{2}{*}{59} \\
\hline$S$ & 152 & 0 & & & & \\
\hline $\mathrm{S}$ & 166 & 100 & \multirow{3}{*}{ 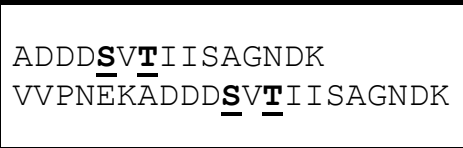 } & \multirow{3}{*}{$\begin{array}{c}121 \\
25\end{array}$} & \multirow{3}{*}{$\begin{array}{l}4.87 \\
4.37\end{array}$} & \multirow{3}{*}{$\begin{array}{c}108 \\
60\end{array}$} \\
\hline $\mathrm{T}$ & 168 & 100 & & & & \\
\hline$S$ & 171 & 90 & & & & \\
\hline $\bar{Y}$ & 250 & 100 & \multirow{3}{*}{$\underline{\mathbf{Y}}$ WLAAATATGIK } & \multirow{3}{*}{21} & \multirow{3}{*}{3.51} & \multirow{3}{*}{56} \\
\hline$T$ & 256 & 0.3 & & & & \\
\hline$T$ & 258 & 0 & & & & \\
\hline$S$ & 291 & 77.4 & \multirow{5}{*}{$\begin{array}{l}\text { AAEPHAVSLAWSADGQTLFAG } \\
\text { YTDNVIR }\end{array}$} & \multirow{5}{*}{17} & \multirow{5}{*}{5.19} & \multirow{5}{*}{91} \\
\hline$S$ & 295 & 93.2 & & & & \\
\hline $\mathrm{T}$ & 300 & 99.9 & & & & \\
\hline $\mathrm{Y}$ & 305 & 0.6 & & & & \\
\hline$T$ & 306 & 0.2 & & & & \\
\hline
\end{tabular}




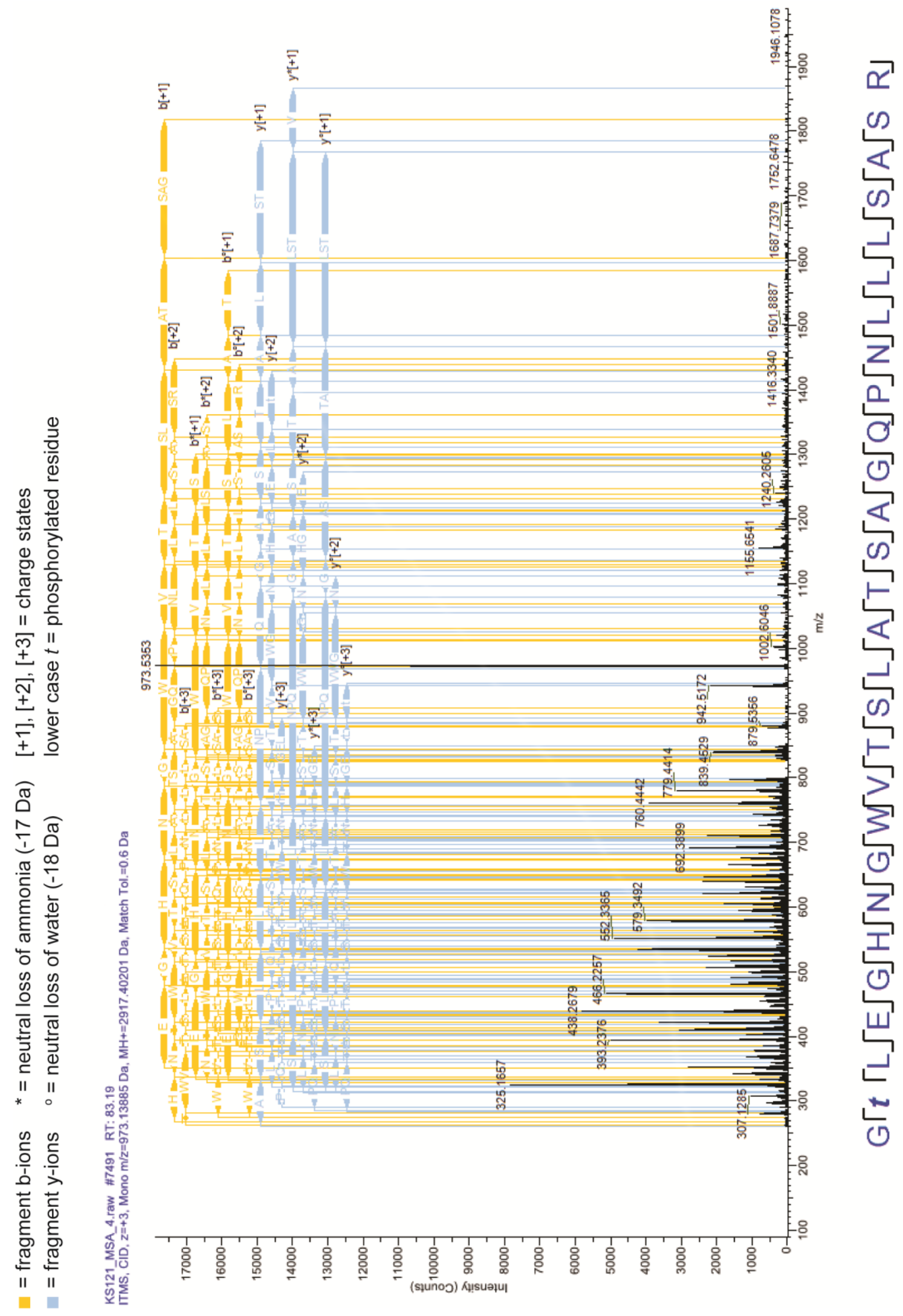

Supplementary Figure 2. Annotated fragmentation spectra of Asc1p phospho-peptides.

(A) Annotated fragmentation spectrum of the peptide GTLEGHNGWVTSLATSAGQPNLLLSASR phosphorylated at $\mathrm{T} 12$. 


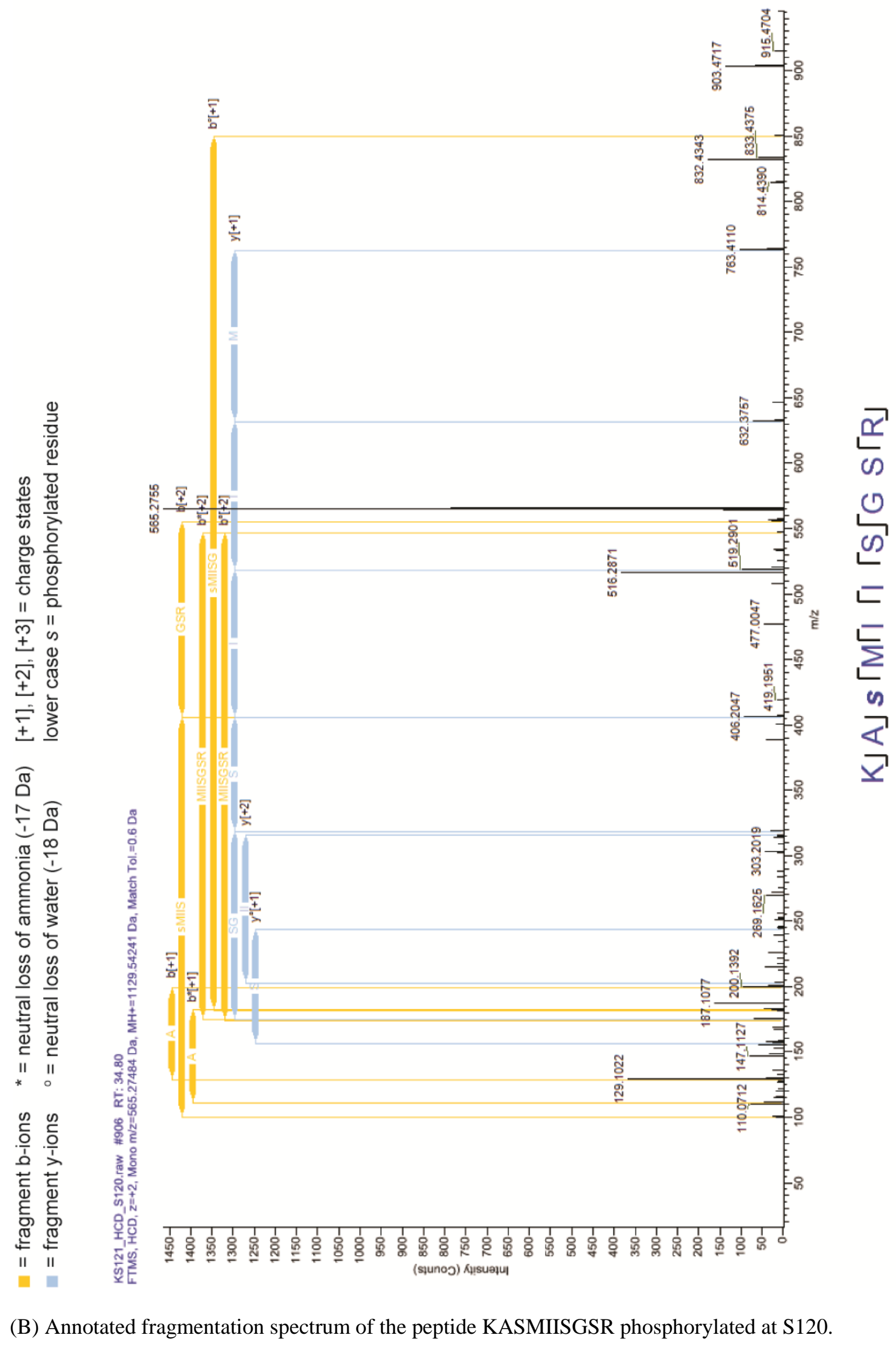




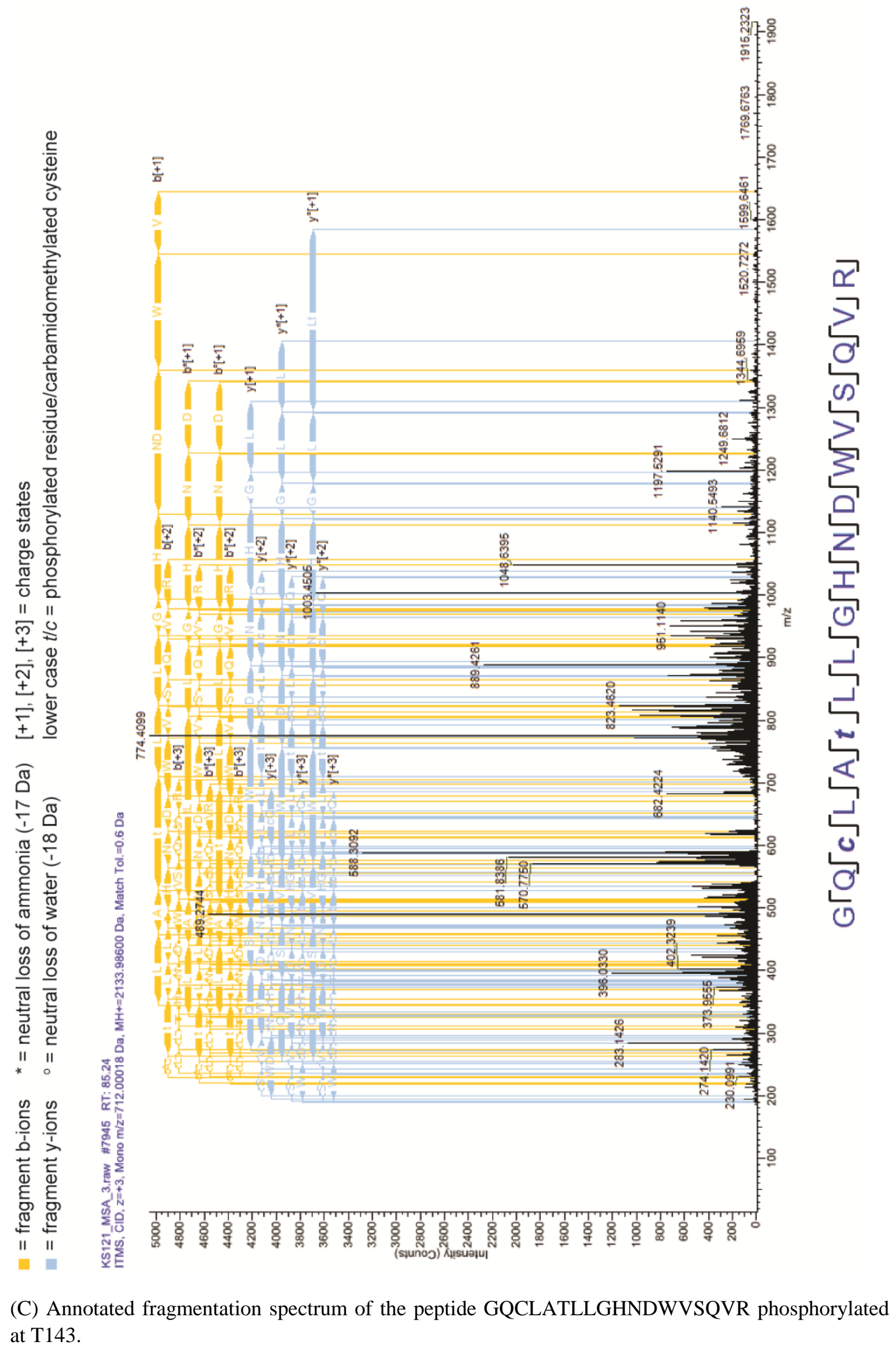




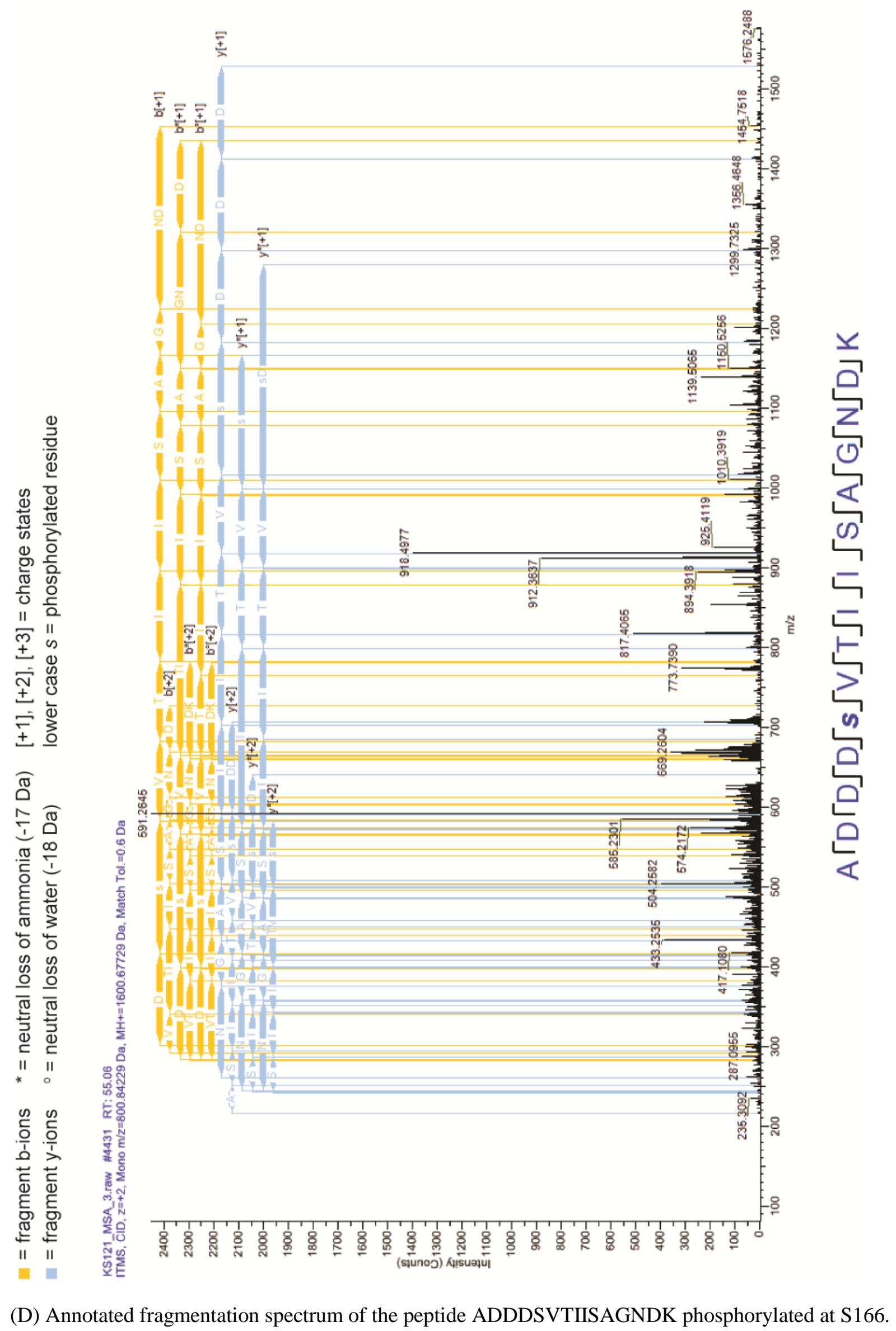




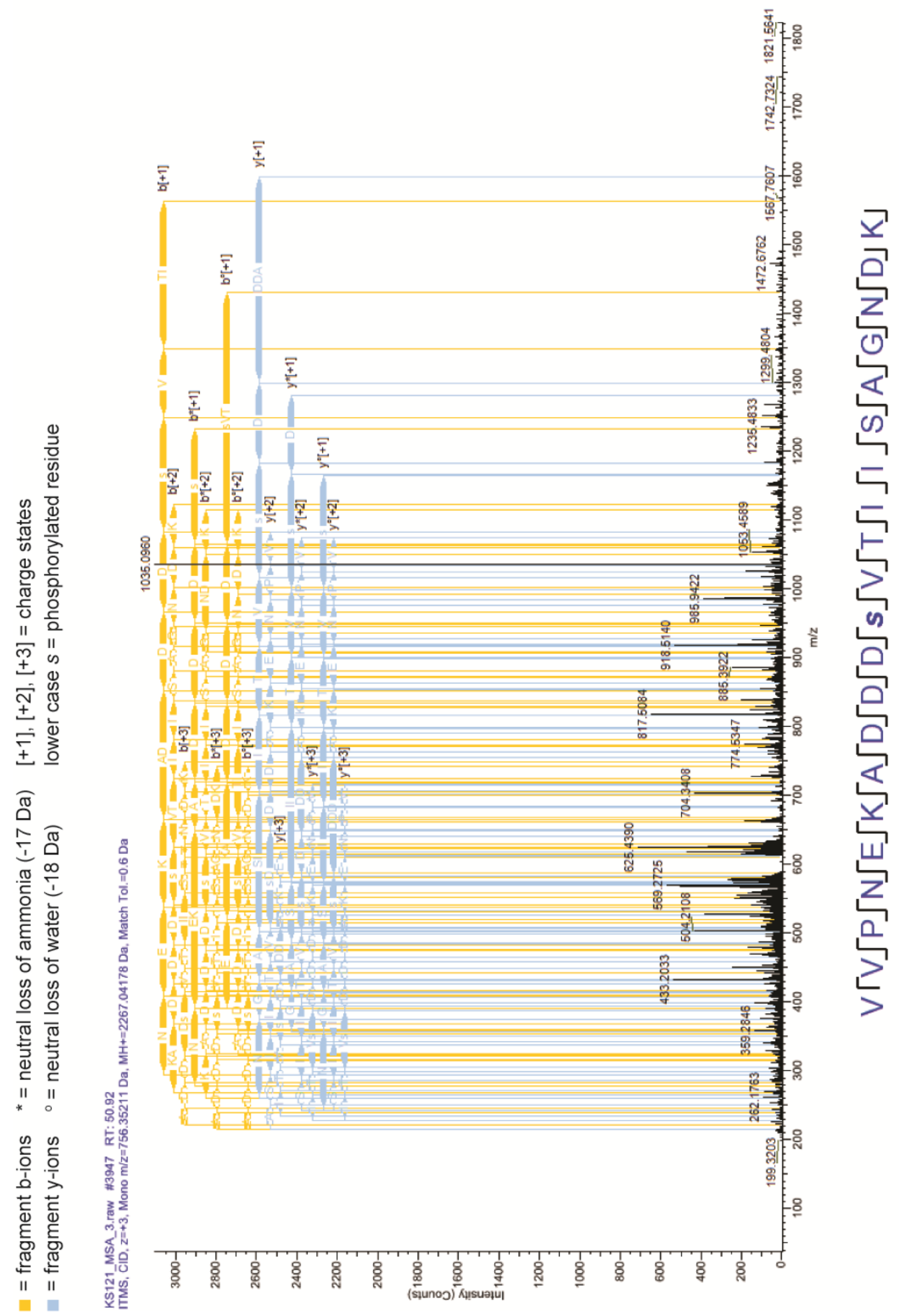

(E) Annotated fragmentation spectrum of the peptide VVPNEKADDDSVTIISAGNDK phosphorylated at S166. 


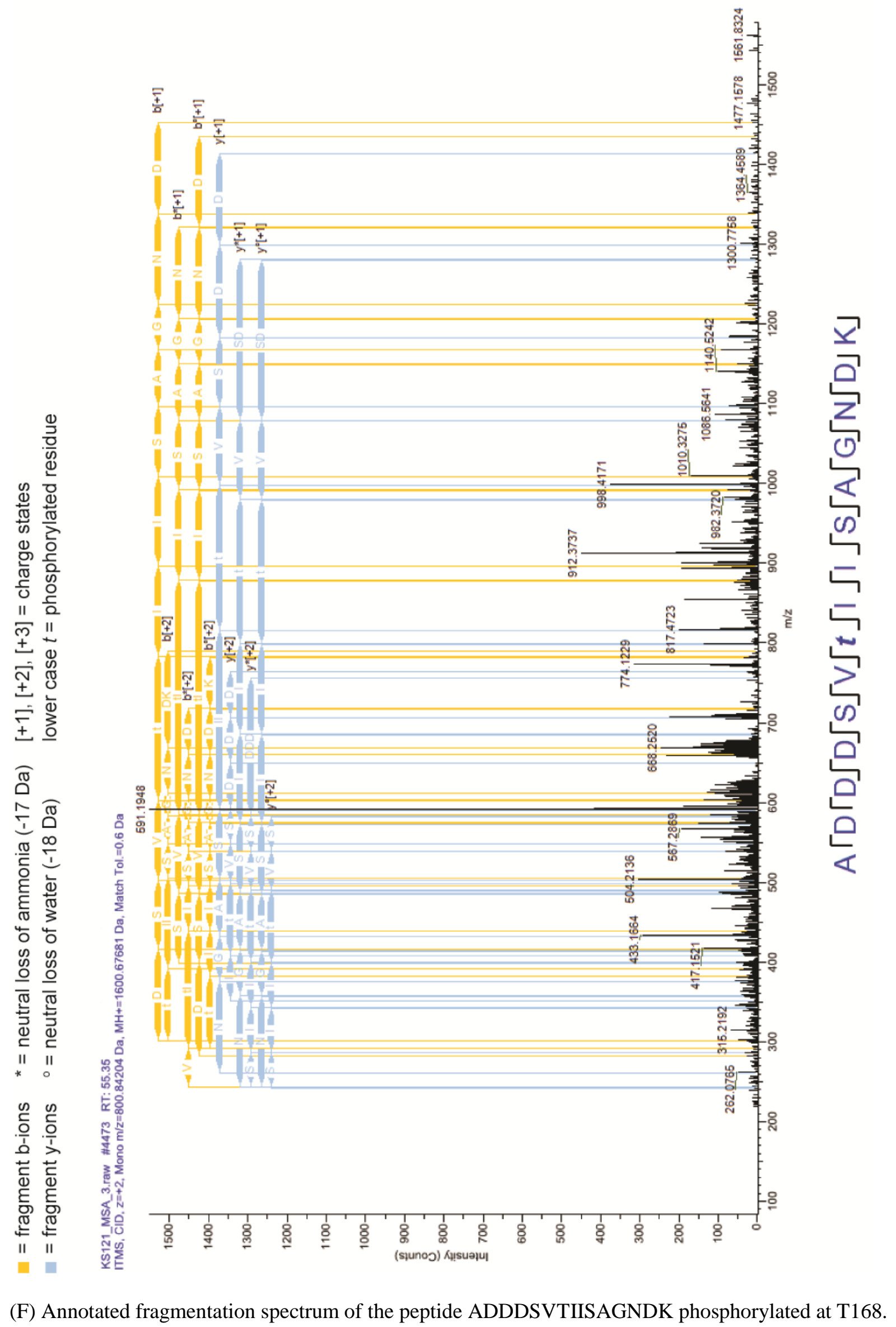




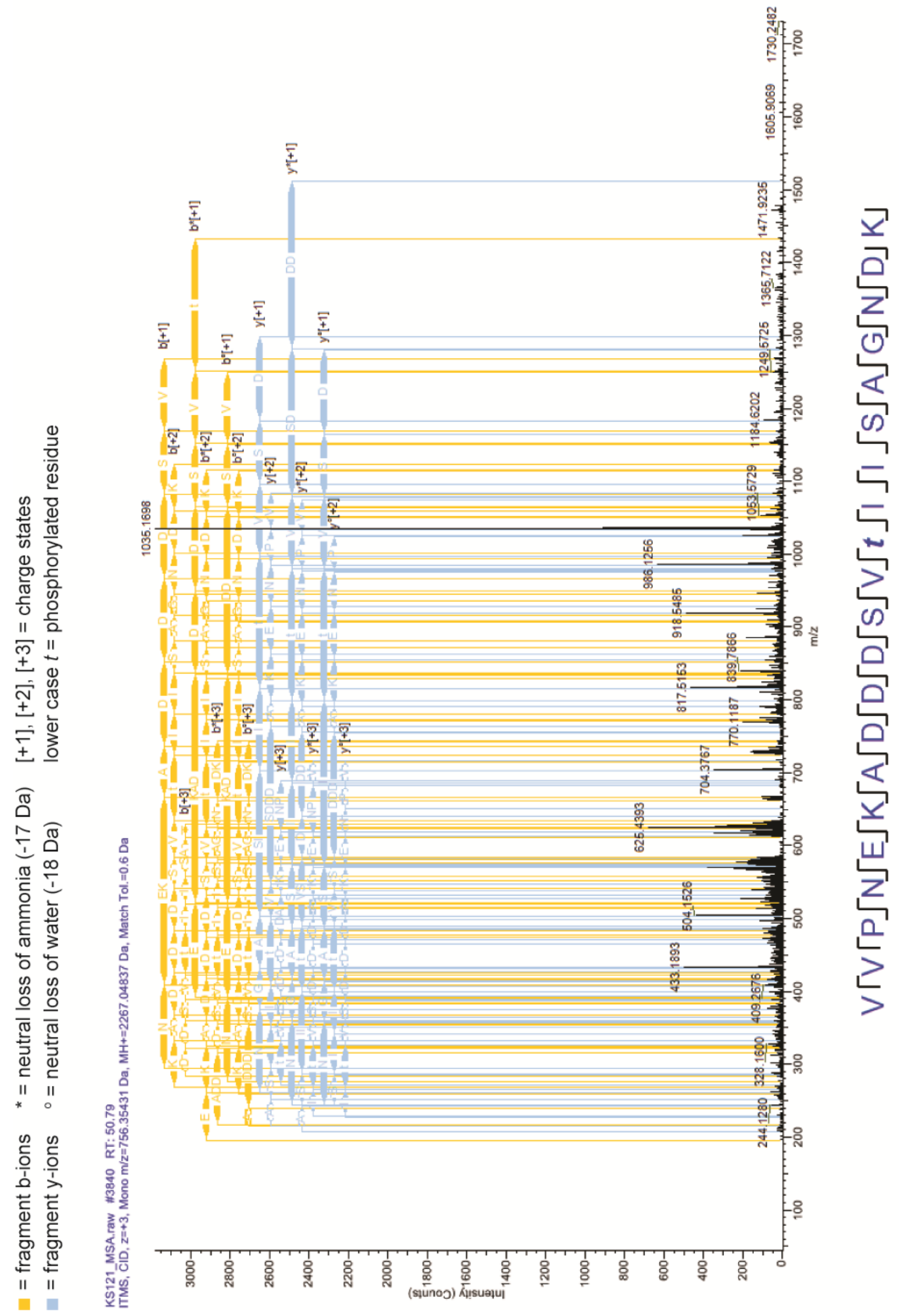

(G) Annotated fragmentation spectrum of the peptide VVPNEKADDDSVTIISAGNDK phosphorylated at T168. 


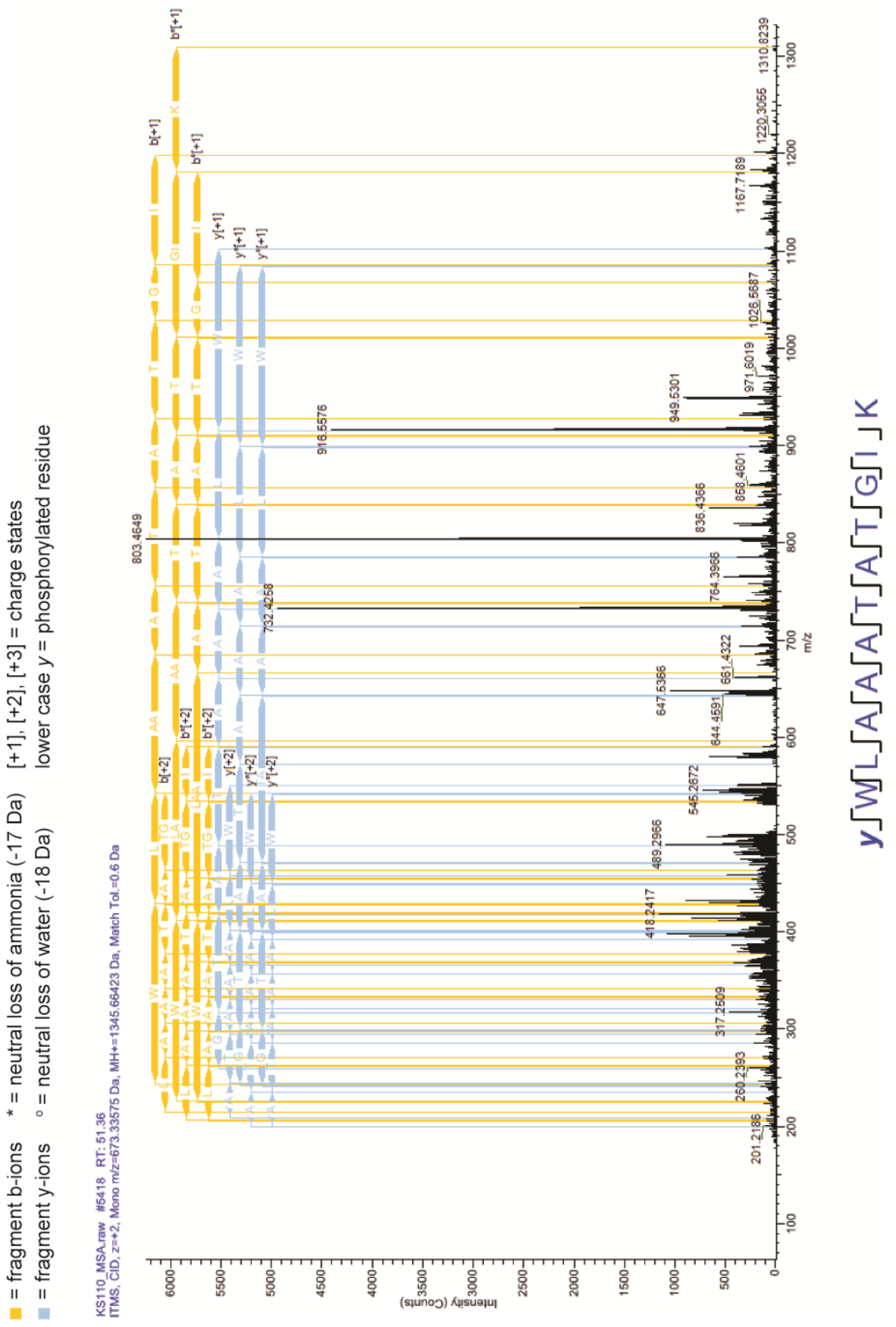

(H) Annotated fragmentation spectrum of the peptide YWLAAATATGIK phosphorylated at Y250. 


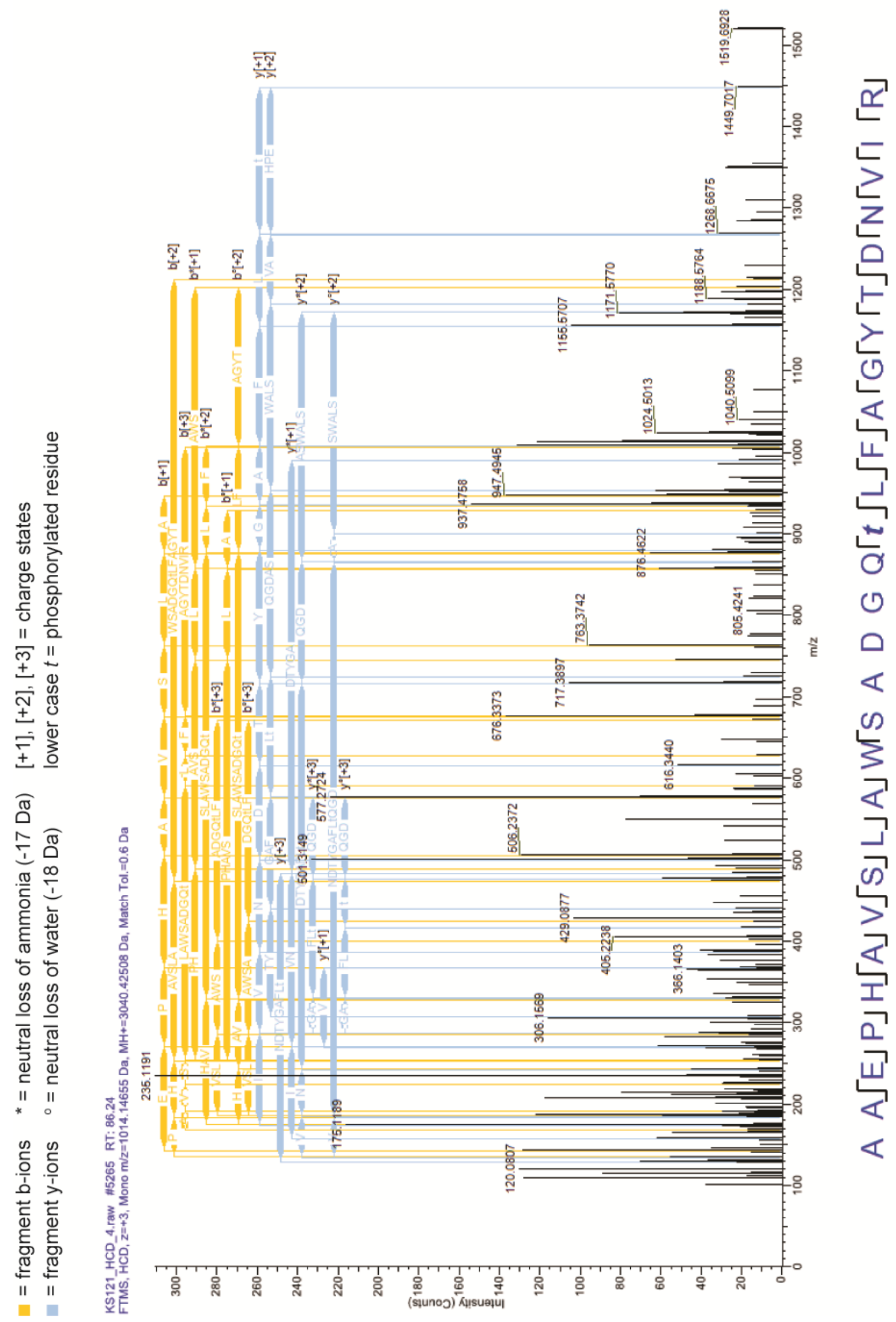

(I) Annotated fragmentation spectrum of the peptide AAEPHAVSLAWSADGQTLFAGYTDNVIR phosphorylated at T300. 
Supplementary Table 2. Overview of proteome data evaluation with Perseus.

Abbreviations: Prot $=$ protein, vs. $=$ versus

\begin{tabular}{|c|c|c|c|}
\hline \multirow[t]{2}{*}{ No. } & \multirow[t]{2}{*}{ Command } & \multicolumn{2}{|l|}{ Description } \\
\hline & & asc1 and asc1DE & $\begin{array}{l}\text { T143 phospho-site mutant } \\
\text { strains, for example, asc } 1^{T 143 A}\end{array}$ \\
\hline 1 & $\begin{array}{l}\text { Generic } \\
\text { matrix upload }\end{array}$ & \multicolumn{2}{|l|}{$\begin{array}{l}\text { proteinGroups.txt } \\
\text { normalized ratios etc. }\end{array}$} \\
\hline $\begin{array}{l}2.1 \\
2.2 \\
2.3\end{array}$ & $\begin{array}{l}\text { Filter rows } \\
\text { based on } \\
\text { categorical } \\
\text { column }\end{array}$ & \multicolumn{2}{|c|}{$\begin{array}{l}\text { Remove rows with }+ \text { in reverse column } \\
\text { Remove rows with }+ \text { in potential contaminant column } \\
\text { Remove rows with }+ \text { in only identified by site column }\end{array}$} \\
\hline 3 & Transform & \multicolumn{2}{|c|}{ Inverse ratios $(1 / \mathrm{x})$ when $A S C 1$ is not in the denominator } \\
\hline 4 & Transform & \multicolumn{2}{|l|}{$\log _{2}(x)$} \\
\hline 5 & Normalization & \multicolumn{2}{|l|}{ Subtract column median of ratios } \\
\hline 6 & $\begin{array}{l}\text { Categorical } \\
\text { annotation } \\
\text { rows }\end{array}$ & \multicolumn{2}{|l|}{ Group biological replicates } \\
\hline 7 & $\begin{array}{l}\text { Reorder/ } \\
\text { remove } \\
\text { columns }\end{array}$ & \multicolumn{2}{|c|}{ Select Prot ratios of interest for the following steps } \\
\hline 8.1 & $\begin{array}{l}\text { Average } \\
\text { groups }\end{array}$ & $\begin{array}{l}\text { Calculate median of each group } \\
\rightarrow \text { protein ratio }\end{array}$ & Calculate mean of each group \\
\hline 8.2 & $\begin{array}{l}\text { Combine } \\
\text { expression } \\
\text { columns }\end{array}$ & - & $\begin{array}{l}\text { Calculation of difference between } \\
\text { mean of asc1 } 1^{\top 143 A} / A S C 1 \text { ratios } \\
\text { and } A S C 1 \text { Aux } / A S C 1 \text { ratios } \\
\rightarrow \text { protein ratio }\end{array}$ \\
\hline 9 & $\begin{array}{l}\text { Change } \\
\text { column type }\end{array}$ & \multicolumn{2}{|c|}{$\begin{array}{l}\text { Change numerical column with protein ratio (steps } 8.1 \text { and } 8.2 \text { ) to } \\
\text { expression column }\end{array}$} \\
\hline 10 & $\begin{array}{l}\text { Categorical } \\
\text { annotation } \\
\text { rows }\end{array}$ & \multicolumn{2}{|c|}{ Define column with protein ratio as own group } \\
\hline 11 & $\begin{array}{l}\text { One/Two } \\
\text { sample(s) } \\
\text { tests }\end{array}$ & $\begin{array}{l}\text { One-sample } t \text {-test, } \\
\text { p-value } 0.01\end{array}$ & $\begin{array}{l}\text { Two-sample } t \text {-test, } \\
\text { p-value } 0.01 \\
\text { Prot ratios asc } 1^{\mathrm{T} 143 \mathrm{~A}} / A S C 1 \\
\text { vs. } \\
\text { Prot ratios } A S C 1 \text { Aux } / A S C 1\end{array}$ \\
\hline 12 & $\begin{array}{l}\text { Filter rows } \\
\text { based on } \\
\text { categorical } \\
\text { column }\end{array}$ & \multicolumn{2}{|c|}{ Keep rows with + (significant) from step 11} \\
\hline 13 & $\begin{array}{l}\text { Filter rows } \\
\text { based on } \\
\text { valid values }\end{array}$ & \multicolumn{2}{|c|}{ Filter protein ratio (steps 8.1 and 8.2 ) for values outside -0.26 to 0.26} \\
\hline
\end{tabular}


Supplementary Table 3. Overview of phospho-proteome data evaluation with Perseus. Abbreviations: $\mathrm{PP}=$ phospho-peptide, Prot $=$ protein, vs. $=$ versus

\begin{tabular}{|c|c|c|c|}
\hline \multirow[t]{2}{*}{ No. } & \multirow[t]{2}{*}{ Command } & \multicolumn{2}{|l|}{ Description } \\
\hline & & asc1- & $\begin{array}{l}\text { T143 phospho-site mutant strains, } \\
\text { for example, asc } 1^{T 143 \mathrm{~A}}\end{array}$ \\
\hline 1 & $\begin{array}{l}\text { Generic } \\
\text { matrix upload }\end{array}$ & \multicolumn{2}{|c|}{$\begin{array}{l}\text { Phospho (STY)Sites.txt } \\
\text { normalized ratios, localization probability etc. }\end{array}$} \\
\hline $\begin{array}{l}2.1 \\
2.2\end{array}$ & $\begin{array}{l}\text { Filter rows } \\
\text { based on } \\
\text { categorical } \\
\text { column }\end{array}$ & \multicolumn{2}{|c|}{$\begin{array}{l}\text { Remove rows with }+ \text { in reverse column } \\
\text { Remove rows with }+ \text { in potential contaminant column }\end{array}$} \\
\hline 3 & $\begin{array}{l}\text { Expand site } \\
\text { table }\end{array}$ & \multicolumn{2}{|c|}{ In order to have only one column per sample } \\
\hline 4 & Transform & \multicolumn{2}{|c|}{ Inverse ratios $(1 / \mathrm{x})$ when $A S C 1$ is not already in the denominator } \\
\hline 5 & Transform & \multicolumn{2}{|l|}{$\log _{2}(x)$} \\
\hline 6 & $\begin{array}{l}\text { Reorder/ } \\
\text { remove } \\
\text { columns }\end{array}$ & \multicolumn{2}{|c|}{ Select PP ratios of interest for the following steps } \\
\hline 7 & $\begin{array}{l}\text { Matching } \\
\text { rows by } \\
\text { name }\end{array}$ & \multicolumn{2}{|c|}{$\begin{array}{l}\text { Matching PP ratios with respective Prot ratios from step } 7 \text { of } \\
\text { Supplementary Table } 2 \text { (in the case that a phospho-peptide cannot be } \\
\text { assigned to a single protein the median of the protein ratios is } \\
\text { calculated) }\end{array}$} \\
\hline 8 & $\begin{array}{l}\text { Categorical } \\
\text { annotation } \\
\text { rows }\end{array}$ & \multicolumn{2}{|c|}{ Group PP ratios and Prot ratios for biological replicates } \\
\hline 9 & $\begin{array}{l}\text { Average } \\
\text { groups }\end{array}$ & Calculate median of each group & Calculate mean of each group \\
\hline 10.1 & \multirow[t]{2}{*}{$\begin{array}{l}\text { Combine } \\
\text { expression } \\
\text { columns }\end{array}$} & \multicolumn{2}{|c|}{$\begin{array}{l}\text { Correction of PP ratios with Prot ratios: } \\
\text { median PP ratios - median Prot ratios } \\
\rightarrow \text { phospho regulation }\end{array}$} \\
\hline 10.2 & & - & $\begin{array}{l}\text { Calculation of difference between } \\
\text { proteome-corrected PP ratios of } \\
\text { asc } 1^{\top 143 \mathrm{~A}} / A S C 1 \text { and } \\
\text { ASC } 1^{\text {Aux }} / A S C 1 \\
\rightarrow \text { phospho regulation }\end{array}$ \\
\hline 11 & $\begin{array}{l}\text { Change } \\
\text { column type }\end{array}$ & \multicolumn{2}{|c|}{$\begin{array}{l}\text { Change numerical column with phospho regulation (steps } 10.1 \text { and } \\
10.2 \text { ) to expression column }\end{array}$} \\
\hline 12 & $\begin{array}{l}\text { Categorical } \\
\text { annotation } \\
\text { rows }\end{array}$ & \multicolumn{2}{|c|}{$\begin{array}{l}\text { Define column with phospho regulation as an own group for later } \\
\text { filtering of values }\end{array}$} \\
\hline 13 & $\begin{array}{l}\text { Categorical } \\
\text { annotation } \\
\text { rows }\end{array}$ & \multicolumn{2}{|c|}{ Group PP ratios for biological replicates for the next step } \\
\hline 14 & $\begin{array}{l}\text { Filter rows } \\
\text { based on } \\
\text { valid values }\end{array}$ & Filter for two valid PP ratios & Filter for one valid PP ratio \\
\hline 15.1 & \multirow[t]{2}{*}{$\begin{array}{l}\text { Two samples } \\
\text { tests }\end{array}$} & $\begin{array}{l}t \text {-test, } \mathrm{p} \text {-value } 0.01 \\
\text { PP ratios vs. Prot ratios }\end{array}$ & $\begin{array}{l}t \text {-test, } p \text {-value } 0.05 \\
\text { PP ratios vs. Prot ratios } \\
\text { for asc1 }{ }^{\mathrm{T} 143 \mathrm{~A}} / A S C 1\end{array}$ \\
\hline 15.2 & & - & $\begin{array}{l}t \text {-test, } \mathrm{p} \text {-value } 0.05 \\
\mathrm{PP} \text { ratios asc1 } 1^{\mathrm{T} 143 \mathrm{~A}} / A S C 1 \mathrm{vs.} \mathrm{PP} \\
\text { ratios } A S C 1^{\mathrm{Aux}} / A S C 1\end{array}$ \\
\hline 16.1 & \multirow{2}{*}{$\begin{array}{l}\text { Filter rows } \\
\text { based on } \\
\text { categorical } \\
\text { column }\end{array}$} & \multicolumn{2}{|c|}{ Keep rows with + (significant) from step 15.1} \\
\hline 16.2 & & - & $\begin{array}{l}\text { Keep rows with + (significant) } \\
\text { from step } 15.2\end{array}$ \\
\hline 17 & $\begin{array}{l}\text { Filter rows } \\
\text { based on } \\
\text { valid values }\end{array}$ & \multicolumn{2}{|c|}{$\begin{array}{l}\text { Filter phospho regulation (steps 10-12) for values outside } \\
-0.26 \text { to } 0.26\end{array}$} \\
\hline
\end{tabular}


Supplementary Table 3. Continued.

\begin{tabular}{|c|c|c|c|}
\hline No. & Command & \multicolumn{2}{|l|}{ Description } \\
\hline \multicolumn{4}{|c|}{$\begin{array}{l}\text { The following steps were only performed for the asc1- phospho-proteome analysis and for } \\
\text { phosphorylated peptides with zero to two corresponding Prot ratios. } \\
\text { Start from step } 15.1\end{array}$} \\
\hline 18 & $\begin{array}{l}\text { One sample } \\
\text { tests }\end{array}$ & \multicolumn{2}{|l|}{$\begin{array}{l}t \text {-test, } \mathrm{p} \text {-value } 0.01 \\
\text { only phospho-peptide ratios }\end{array}$} \\
\hline 19 & $\begin{array}{l}\text { Filter rows } \\
\text { based on } \\
\text { categorical } \\
\text { column }\end{array}$ & \multicolumn{2}{|c|}{$\begin{array}{l}\text { Discard rows with + (significant) from step } 15.1 \text { (these candidates } \\
\text { showed regulation of PP ratios with respect to Prot values) }\end{array}$} \\
\hline 20 & $\begin{array}{l}\text { Filter rows } \\
\text { based on } \\
\text { categorical } \\
\text { column }\end{array}$ & \multicolumn{2}{|c|}{ Keep rows with + (significant) from step 18} \\
\hline 21 & $\begin{array}{l}\text { Categorical } \\
\text { annotation } \\
\text { rows }\end{array}$ & \multicolumn{2}{|l|}{$\begin{array}{l}\text { Group Prot ratios } \\
\rightarrow \text { proteome }\end{array}$} \\
\hline 22 & $\begin{array}{l}\text { Filter rows } \\
\text { based on } \\
\text { valid values }\end{array}$ & \multicolumn{2}{|c|}{$\begin{array}{l}\text { Filter rows based on three valid values in the proteome group, add } \\
\text { categorical column (assumption: less than three valid values might be } \\
\text { insufficient for two-samples } t \text {-test) }\end{array}$} \\
\hline 23 & $\begin{array}{l}\text { Filter rows } \\
\text { based on } \\
\text { categorical } \\
\text { column }\end{array}$ & \multicolumn{2}{|c|}{$\begin{array}{l}\text { Keep rows that were specified as discarded in the previous step } \\
\text { (remaining candidates have less than three valid values in the } \\
\text { proteome group) }\end{array}$} \\
\hline 24 & $\begin{array}{l}\text { Filter rows } \\
\text { based on } \\
\text { valid values }\end{array}$ & \multicolumn{2}{|c|}{$\begin{array}{l}\text { Filter rows based on one valid value in proteome group, add } \\
\text { categorical column }\end{array}$} \\
\hline & & $\begin{array}{l}\text { phosphorylated peptides with no } \\
\text { corresponding Prot ratio }\end{array}$ & $\begin{array}{l}\text { phosphorylated peptides with one } \\
\text { or two corresponding Prot ratio(s) }\end{array}$ \\
\hline 25 & $\begin{array}{l}\text { Filter rows } \\
\text { based on } \\
\text { categorical } \\
\text { column }\end{array}$ & $\begin{array}{l}\text { Keep rows that were specified as } \\
\text { discarded in the previous step, } \\
\text { they have no proteome values }\end{array}$ & $\begin{array}{l}\text { Keep rows that were specified as } \\
\text { keep in the previous step, they } \\
\text { have one or two proteome } \\
\text { value(s) }\end{array}$ \\
\hline 26 & $\begin{array}{l}\text { Change } \\
\text { column type }\end{array}$ & $\begin{array}{l}\text { Change numerical column with } \\
\text { PP median ratio (step 9) to } \\
\text { expression column }\end{array}$ & - \\
\hline 27 & $\begin{array}{l}\text { Categorical } \\
\text { annotation } \\
\text { rows }\end{array}$ & $\begin{array}{l}\text { Define column with PP median } \\
\text { ratio from the previous step as an } \\
\text { own group for filtering of values in } \\
\text { the next step } \\
\rightarrow \text { phospho regulation with no } \\
\text { Prot value }\end{array}$ & - \\
\hline 28 & $\begin{array}{l}\text { Filter rows } \\
\text { based on } \\
\text { valid values }\end{array}$ & $\begin{array}{l}\text { Filter phospho regulation with no } \\
\text { Prot value (step } 27 \text { ) for values } \\
\text { outside }-0.26 \text { to } 0.26\end{array}$ & $\begin{array}{l}\text { Filter phospho regulation (step } \\
10.1 \text { ) for values outside } \\
-0.26 \text { to } 0.26\end{array}$ \\
\hline
\end{tabular}

Color scale for Supplementary Tables 4-11, 15, and 16

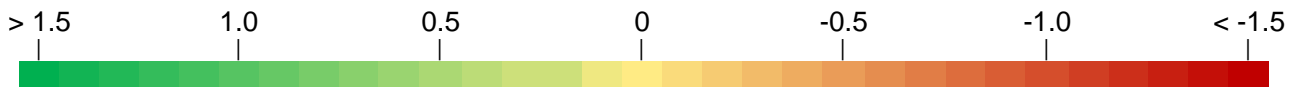


Supplementary Table 4. SILAC-based proteome data for the comparison of the asc $1^{-}$strain and the $A S C 1$ wild-type strain.

The table lists proteins with a median asc1 $/$ ASC1 $\log _{2}$ SILAC-ratio $\leq-0.58$ or $\geq 0.58$ and a p-value $<0.01$. Proteins with SILAC-ratios $>0.26$ or $<-0.26$ are not shown. Replicates are numbered 1-8 and

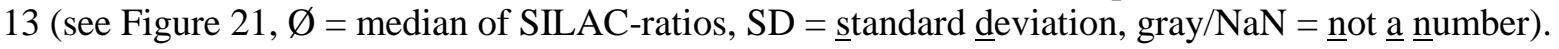

\begin{tabular}{|c|c|c|c|c|c|c|c|c|c|c|c|c|}
\hline \multirow{2}{*}{ proteins } & \multirow{2}{*}{$\begin{array}{l}\text { protein } \\
\text { IDs }\end{array}$} & \multicolumn{9}{|c|}{ asc1-/ASC1 } & \multirow[b]{2}{*}{$\varnothing$} & \multirow[b]{2}{*}{ SD } \\
\hline & & 1 & 2 & 3 & 4 & 5 & 6 & 7 & 8 & 13 & & \\
\hline Aro9 & P38840 & 1.70 & 2.00 & 2.15 & $\mathrm{NaN}$ & $\mathrm{NaN}$ & $\mathrm{NaN}$ & $\mathrm{NaN}$ & $\mathrm{NaN}$ & 1.75 & 1.88 & 0.21 \\
\hline Hbn1 & Q96VH4 & 1.27 & 1.15 & 1.27 & 1.58 & 1.50 & 1.85 & 2.01 & 1.65 & 1.26 & 1.50 & 0.30 \\
\hline Yhb1 & P39676 & 1.32 & 1.26 & 1.28 & 1.43 & 1.63 & 1.52 & 1.62 & 1.68 & 1.29 & 1.43 & 0.17 \\
\hline Bna2 & P47125 & $\mathrm{NaN}$ & $\mathrm{NaN}$ & 0.96 & 0.99 & 1.40 & 1.39 & 1.43 & 1.56 & 1.21 & 1.39 & 0.23 \\
\hline Sam3 & Q08986 & $\mathrm{NaN}$ & $\mathrm{NaN}$ & 1.57 & 1.10 & 1.01 & $\mathrm{NaN}$ & 1.50 & 1.39 & 1.38 & 1.38 & 0.22 \\
\hline Ty1a $^{1}$ & P0CX58 ${ }^{1}$ & $\mathrm{NaN}$ & $\mathrm{NaN}$ & 0.64 & $\mathrm{NaN}$ & $\mathrm{NaN}$ & 1.33 & 0.91 & 1.34 & 1.33 & 1.33 & 0.32 \\
\hline Ty2b ${ }^{2}$ & P25384 ${ }^{2}$ & 0.63 & 1.18 & 0.93 & 0.82 & 1.68 & $\mathrm{NaN}$ & 1.63 & 1.21 & $\mathrm{NaN}$ & 1.18 & 0.40 \\
\hline Ty1b3 & Q03612 3 & 1.07 & 1.11 & 1.10 & 1.12 & 1.39 & 1.31 & 1.29 & 1.32 & 1.03 & 1.12 & 0.13 \\
\hline Hsp82 & P02829 & 1.02 & 1.13 & 1.04 & 0.90 & 0.93 & 1.36 & 1.17 & 1.11 & 1.13 & 1.11 & 0.14 \\
\hline Ty1a & P0CX73 & 1.13 & 1.01 & 1.10 & $\mathrm{NaN}$ & $\mathrm{NaN}$ & $\mathrm{NaN}$ & 1.44 & 1.58 & 1.06 & 1.11 & 0.24 \\
\hline Ych1 & P42937 & 1.14 & 1.16 & 1.09 & 0.81 & 1.06 & 0.71 & 1.43 & 1.05 & 1.05 & 1.06 & 0.21 \\
\hline Mtd1 & Q02046 & 1.03 & 0.81 & 0.86 & 1.12 & 1.20 & 0.85 & 1.13 & 1.11 & 0.85 & 1.03 & 0.15 \\
\hline Sui1 & P32911 & 0.89 & 1.01 & 1.02 & 1.02 & 1.03 & 0.91 & 1.02 & 1.06 & 0.78 & 1.02 & 0.09 \\
\hline Ty2b ${ }^{5}$ & Q123375 & 0.84 & 0.94 & 0.87 & 0.93 & 1.89 & 1.51 & 1.86 & 1.68 & 0.67 & 0.94 & 0.48 \\
\hline Dak1 & P54838 & 0.89 & 0.99 & 0.88 & 0.72 & 0.81 & 1.22 & 0.99 & 0.94 & 0.94 & 0.94 & 0.14 \\
\hline Hsp104 & P31539 & 0.89 & 0.93 & 0.80 & 0.73 & 0.82 & 1.30 & 1.09 & 1.01 & 1.02 & 0.93 & 0.17 \\
\hline Sro9 & P25567 & 0.92 & 0.80 & 0.80 & 0.85 & 1.00 & 1.15 & 1.01 & 0.99 & 0.70 & 0.92 & 0.14 \\
\hline Ren2 & Q12044 & $\mathrm{NaN}$ & 0.85 & 0.81 & $\mathrm{NaN}$ & 0.94 & $\mathrm{NaN}$ & $\mathrm{NaN}$ & 0.92 & 1.01 & 0.92 & 0.08 \\
\hline Dcs1 & Q06151 & 1.07 & 0.69 & 0.86 & 0.73 & 0.83 & 1.07 & 0.79 & 1.07 & 0.92 & 0.86 & 0.15 \\
\hline Aap1 & P37898 & 0.85 & 0.35 & 1.01 & 0.96 & 0.58 & 0.75 & 0.87 & 0.93 & 0.78 & 0.85 & 0.21 \\
\hline Gly1 & P37303 & 0.85 & 0.75 & 0.88 & 0.95 & 0.99 & 0.78 & 0.88 & 0.79 & 0.74 & 0.85 & 0.09 \\
\hline Nmd5 & P46970 & 0.86 & 0.68 & 0.69 & 0.79 & 1.07 & 0.88 & 0.98 & 0.84 & 0.76 & 0.84 & 0.13 \\
\hline Fpr4 & Q06205 & 0.05 & 0.92 & 0.66 & 0.87 & 1.05 & 1.11 & 0.60 & 0.74 & 0.84 & 0.84 & 0.32 \\
\hline Lst8 & P41318 & 0.31 & 0.93 & $\mathrm{NaN}$ & 0.81 & $\mathrm{NaN}$ & $\mathrm{NaN}$ & 0.88 & 0.91 & 0.34 & 0.84 & 0.29 \\
\hline Ssa1 & P10591 & 0.87 & 0.91 & 0.84 & 0.67 & 0.63 & 1.08 & 0.81 & 0.76 & 0.91 & 0.84 & 0.14 \\
\hline Lap3 & Q01532 & 0.77 & 0.83 & 0.80 & 0.71 & 0.88 & 1.10 & 1.14 & 0.98 & 0.73 & 0.83 & 0.16 \\
\hline Cue5 & Q08412 & $\mathrm{NaN}$ & 0.53 & 0.88 & 0.79 & $\mathrm{NaN}$ & 1.11 & 0.71 & 0.88 & $\mathrm{NaN}$ & 0.83 & 0.19 \\
\hline Fmp41 & P53889 & 0.55 & 0.69 & 0.89 & 0.76 & 0.92 & 1.02 & 1.16 & $\mathrm{NaN}$ & 0.59 & 0.82 & 0.21 \\
\hline Bna1 & P47096 & 0.82 & 0.79 & 0.77 & 0.83 & 1.02 & 0.74 & 1.03 & 0.99 & 0.71 & 0.82 & 0.13 \\
\hline Cex1 & Q12453 & 0.89 & 0.90 & 0.64 & 0.81 & 0.89 & 1.07 & 0.76 & 0.78 & 0.69 & 0.81 & 0.13 \\
\hline Aro7 & P32178 & 0.34 & 0.69 & 0.80 & 0.99 & 0.96 & 0.87 & 0.67 & 0.99 & 0.48 & 0.80 & 0.23 \\
\hline Hem15 & P16622 & 0.69 & 0.81 & 0.73 & 0.80 & 0.82 & 0.99 & 0.79 & 0.65 & 0.57 & 0.79 & 0.12 \\
\hline Ddi1 & P40087 & 0.75 & 0.78 & 0.80 & 0.95 & 0.71 & 1.02 & 0.77 & 0.81 & 0.67 & 0.78 & 0.11 \\
\hline Nbp2 & Q12163 & $\mathrm{NaN}$ & $\mathrm{NaN}$ & 0.83 & 0.78 & $\mathrm{NaN}$ & 0.69 & $\mathrm{NaN}$ & $\mathrm{NaN}$ & $\mathrm{NaN}$ & 0.78 & 0.07 \\
\hline Gpm2 & Q12008 & 0.92 & 0.66 & 0.58 & 0.43 & 0.96 & 0.99 & 0.78 & 1.13 & 0.70 & 0.78 & 0.22 \\
\hline Abp1 & P15891 & 0.85 & 0.78 & 0.77 & 0.83 & 0.55 & 0.98 & 0.52 & 0.56 & 0.87 & 0.78 & 0.16 \\
\hline Sti1 & P15705 & 0.68 & 0.80 & 0.76 & 0.67 & 0.70 & 0.83 & 0.82 & 0.73 & 0.78 & 0.76 & 0.06 \\
\hline Plb1 & P39105 & 0.72 & 0.59 & 0.52 & 0.72 & 0.88 & 0.77 & 0.90 & 0.79 & 0.75 & 0.75 & 0.12 \\
\hline Atg18 & P43601 & $\mathrm{NaN}$ & $\mathrm{NaN}$ & 0.76 & $\mathrm{NaN}$ & $\mathrm{NaN}$ & $\mathrm{NaN}$ & 0.74 & $\mathrm{NaN}$ & $\mathrm{NaN}$ & 0.75 & 0.01 \\
\hline His7 & P33734 & 0.71 & 0.74 & 0.72 & 0.70 & 0.77 & 0.72 & 0.75 & 0.79 & 0.61 & 0.72 & 0.05 \\
\hline Fdc1 & Q03034 & 0.93 & 0.22 & 0.58 & 0.68 & $\mathrm{NaN}$ & 0.86 & 1.07 & 0.75 & 0.43 & 0.72 & 0.28 \\
\hline
\end{tabular}


Supplementary Table 4. Continued 1.

\begin{tabular}{|c|c|c|c|c|c|c|c|c|c|c|c|c|}
\hline \multirow{2}{*}{ proteins } & \multirow{2}{*}{$\begin{array}{l}\text { protein } \\
\text { IDs }\end{array}$} & \multicolumn{9}{|c|}{$\operatorname{asc1/ASC1}$} & \multirow[b]{2}{*}{$\varnothing$} & \multirow[b]{2}{*}{ SD } \\
\hline & & 1 & 2 & 3 & 4 & 5 & 6 & 7 & 8 & 13 & & \\
\hline Cki1 & P20485 & 0.66 & 0.62 & 0.77 & 0.63 & 0.61 & 0.88 & 0.78 & 0.82 & 0.72 & 0.72 & 0.10 \\
\hline Grx3 & Q03835 & 0.56 & 0.71 & 0.66 & 0.72 & 0.84 & 0.96 & 0.64 & 0.83 & 0.77 & 0.72 & 0.12 \\
\hline Lap4 & P14904 & 0.78 & 0.69 & 0.71 & 0.62 & 0.69 & 1.06 & 0.89 & 0.85 & 0.70 & 0.71 & 0.14 \\
\hline Twf1 & P53250 & 0.67 & 0.71 & 0.77 & 0.62 & 0.71 & 0.90 & 0.67 & 0.67 & 0.74 & 0.71 & 0.08 \\
\hline Aip1 & P46680 & 0.83 & 0.78 & 0.69 & 0.69 & 0.60 & 0.76 & 0.71 & 0.67 & 0.82 & 0.71 & 0.07 \\
\hline Ubc1 & P21734 & 0.75 & 0.69 & 0.72 & 0.70 & 0.57 & 0.68 & 0.70 & 0.56 & 0.77 & 0.70 & 0.07 \\
\hline Cdc28 & P00546 & 0.54 & 0.60 & 0.70 & 0.52 & 0.89 & 0.74 & 0.78 & 0.89 & 0.61 & 0.70 & 0.14 \\
\hline $\mathrm{Bbc1}$ & P47068 & 0.64 & 0.52 & 0.79 & 0.57 & 0.69 & 0.89 & 0.77 & 0.35 & 0.86 & 0.69 & 0.17 \\
\hline Hsp42 & Q12329 & 0.63 & 1.04 & 0.51 & 0.69 & 0.56 & 1.49 & 0.55 & 0.77 & 1.23 & 0.69 & 0.35 \\
\hline Ato3 & Q12359 & $\mathrm{NaN}$ & $\mathrm{NaN}$ & 0.59 & $\mathrm{NaN}$ & $\mathrm{NaN}$ & $\mathrm{NaN}$ & 0.76 & 0.69 & $\mathrm{NaN}$ & 0.69 & 0.08 \\
\hline Nfu1 & P32860 & 0.48 & 0.71 & 0.68 & 0.89 & 0.56 & 0.70 & 0.53 & 0.67 & 0.77 & 0.68 & 0.13 \\
\hline Ald2 & P47771 & 0.27 & 0.78 & 0.33 & $\mathrm{NaN}$ & 0.62 & 1.03 & 0.74 & $\mathrm{NaN}$ & $\mathrm{NaN}$ & 0.68 & 0.29 \\
\hline Rbg2 & P53295 & 0.62 & 0.45 & 0.41 & 0.37 & 0.90 & 0.68 & 0.76 & 0.69 & 1.00 & 0.68 & 0.22 \\
\hline Ycr016w & P25617 & 0.88 & 0.70 & 0.49 & 0.66 & $\mathrm{NaN}$ & $\mathrm{NaN}$ & 0.75 & $\mathrm{NaN}$ & 0.24 & 0.68 & 0.22 \\
\hline Eno1 & P00924 & 0.68 & 0.58 & 0.36 & 0.36 & 0.80 & 0.63 & 0.95 & 0.89 & 0.67 & 0.67 & 0.21 \\
\hline Trp3 & P00937 & 0.57 & 0.59 & 0.66 & 0.61 & 0.84 & 0.72 & 0.82 & 0.74 & 0.67 & 0.67 & 0.10 \\
\hline Aro8 & P53090 & 0.68 & 0.68 & 0.60 & 0.64 & 0.64 & 0.67 & 0.72 & 0.64 & 0.68 & 0.67 & 0.04 \\
\hline Acs2 & P52910 & 0.67 & 0.60 & 0.66 & 0.67 & 0.75 & 0.70 & 0.80 & 0.75 & 0.60 & 0.67 & 0.07 \\
\hline Krs1 & P15180 & 0.60 & 0.67 & 0.61 & 0.58 & 0.71 & 0.69 & 0.75 & 0.70 & 0.63 & 0.67 & 0.06 \\
\hline Ypt52 & P36018 & 0.66 & 0.55 & 0.63 & 0.45 & 0.70 & 0.59 & 0.75 & 0.66 & 0.68 & 0.66 & 0.09 \\
\hline Ent5 & Q03769 & 0.17 & 0.47 & 0.25 & 0.82 & 0.78 & 0.79 & 0.66 & 0.76 & 0.56 & 0.66 & 0.24 \\
\hline Pan6 & P40459 & 1.12 & 0.35 & 0.66 & 0.81 & 0.57 & 0.48 & 0.68 & 0.75 & 0.58 & 0.66 & 0.22 \\
\hline Sis1 & P25294 & 0.62 & 0.53 & 0.58 & 0.49 & 0.75 & 0.67 & 0.65 & 0.72 & 0.67 & 0.65 & 0.08 \\
\hline Npa3 & P47122 & 0.60 & 0.75 & 0.64 & 0.70 & 0.65 & 0.40 & 0.67 & 0.75 & 0.59 & 0.65 & 0.11 \\
\hline Trm5 & P38793 & 0.59 & 0.65 & 0.15 & $\mathrm{NaN}$ & 1.03 & 0.43 & 0.69 & 0.62 & 0.66 & 0.63 & 0.25 \\
\hline Rio2 & P40160 & 0.44 & $\mathrm{NaN}$ & 0.59 & $\mathrm{NaN}$ & 0.40 & 0.70 & 0.72 & 0.67 & $\mathrm{NaN}$ & 0.63 & 0.14 \\
\hline Car1 & P00812 & 0.57 & 0.28 & 0.63 & 0.63 & 0.65 & 0.63 & 0.59 & 0.70 & 0.51 & 0.63 & 0.12 \\
\hline Ade1 & P27616 & 0.63 & 0.63 & 0.58 & 0.58 & 0.87 & 0.62 & 0.84 & 0.91 & 0.61 & 0.63 & 0.14 \\
\hline His5 & P07172 & 0.59 & 0.62 & 0.59 & 0.60 & 0.67 & 0.64 & 0.69 & 0.71 & 0.58 & 0.62 & 0.05 \\
\hline Aro3 & P14843 & 0.64 & 0.59 & 0.53 & 0.50 & 0.67 & 0.62 & 0.79 & 0.66 & 0.59 & 0.62 & 0.08 \\
\hline Aim17 & P23180 & 0.71 & 0.56 & 0.54 & 0.48 & 0.10 & 1.05 & 0.62 & 0.74 & 0.93 & 0.62 & 0.27 \\
\hline Bna5 & Q05979 & 0.01 & 0.83 & 0.59 & 0.62 & 0.94 & 0.61 & 0.92 & 1.19 & 0.50 & 0.62 & 0.34 \\
\hline Trm82 & Q03774 & 0.37 & 0.32 & 0.61 & $\mathrm{NaN}$ & 0.66 & 0.77 & $\mathrm{NaN}$ & 0.65 & 0.36 & 0.61 & 0.18 \\
\hline Glr1 & P41921 & 0.55 & 0.63 & 0.57 & 0.62 & 0.43 & 0.61 & 0.81 & 0.67 & 0.58 & 0.61 & 0.10 \\
\hline Cys3 & P31373 & 0.61 & 0.55 & 0.54 & 0.61 & 0.62 & 0.68 & 0.67 & 0.69 & 0.55 & 0.61 & 0.06 \\
\hline Trp5 & P00931 & 0.58 & 0.60 & 0.63 & 0.59 & 0.59 & 0.60 & 0.78 & 0.64 & 0.61 & 0.60 & 0.06 \\
\hline Erg26 & P53199 & 0.54 & 0.57 & 0.60 & 0.74 & 0.77 & 0.61 & 0.57 & 0.63 & 0.43 & 0.60 & 0.10 \\
\hline Faa4 & P47912 & 0.60 & 0.86 & 0.35 & 0.49 & 0.72 & 0.44 & 0.72 & 0.80 & 0.32 & 0.60 & 0.20 \\
\hline Cpa2 & P03965 & 0.58 & 0.75 & 0.73 & 0.62 & 0.43 & 0.51 & 0.59 & 0.54 & 0.64 & 0.59 & 0.10 \\
\hline Tps1 & Q00764 & 0.63 & 0.66 & 0.58 & 0.31 & 0.25 & 0.73 & 0.45 & 0.41 & 0.69 & 0.58 & 0.18 \\
\hline Frd1 & P32614 & 0.58 & 0.51 & 0.55 & 0.60 & 0.72 & 0.54 & 0.79 & 0.65 & 0.42 & 0.58 & 0.11 \\
\hline Gcn3 & P14741 & 0.66 & 0.58 & 0.58 & 0.51 & 0.36 & 0.58 & 0.58 & 0.53 & 0.50 & 0.58 & 0.08 \\
\hline Rrp1 & P35178 & 0.34 & 0.68 & 0.57 & 0.58 & 0.67 & 0.57 & 0.63 & 0.63 & 0.54 & 0.58 & 0.10 \\
\hline Ahp1 & P38013 & -0.41 & -0.41 & -0.45 & -0.58 & -0.94 & -0.60 & -0.81 & -0.81 & -0.42 & -0.58 & 0.20 \\
\hline
\end{tabular}


Supplementary Table 4. Continued 2.

\begin{tabular}{|c|c|c|c|c|c|c|c|c|c|c|c|c|}
\hline \multirow{2}{*}{ proteins } & \multirow{2}{*}{$\begin{array}{l}\text { protein } \\
\text { IDs }\end{array}$} & \multicolumn{9}{|c|}{$\operatorname{asc} 1 / A S C 1$} & \multirow[b]{2}{*}{$\varnothing$} & \multirow[b]{2}{*}{ SD } \\
\hline & & 1 & 2 & 3 & 4 & 5 & 6 & 7 & 8 & 13 & & \\
\hline Lys4 & P49367 & -0.65 & -0.69 & -0.71 & -0.47 & -0.50 & -0.75 & -0.43 & -0.58 & -0.51 & -0.58 & 0.12 \\
\hline Tom70 & P07213 & -0.59 & -0.51 & -0.57 & -0.58 & -0.68 & -0.47 & -0.62 & -0.62 & -0.57 & -0.58 & 0.06 \\
\hline Hhf1 & P02309 & -0.50 & -0.51 & -0.61 & -0.60 & -0.59 & -0.70 & -0.59 & -0.62 & -0.56 & -0.59 & 0.06 \\
\hline Pim1 & P36775 & -0.68 & -0.48 & -0.59 & -0.64 & -0.54 & -0.59 & -0.58 & -0.65 & -0.53 & -0.59 & 0.06 \\
\hline Sec53 & P07283 & -0.57 & -0.59 & -0.60 & -0.66 & -0.61 & -0.59 & -0.63 & -0.54 & -0.55 & -0.59 & 0.04 \\
\hline Dcp2 & P53550 & -0.76 & -0.94 & -0.65 & -0.79 & -0.31 & -0.21 & $\mathrm{NaN}$ & -0.56 & -0.53 & -0.60 & 0.25 \\
\hline Hem13 & P11353 & $\mathrm{NaN}$ & -0.72 & -0.75 & -0.40 & -0.31 & -0.66 & -0.66 & -0.29 & -0.57 & -0.61 & 0.19 \\
\hline Mam33 & P40513 & -0.84 & -0.50 & -0.39 & -0.73 & -1.04 & -0.13 & $\mathrm{NaN}$ & -0.46 & -0.83 & -0.61 & 0.30 \\
\hline Pmt2 & P31382 & -0.61 & -0.66 & -0.59 & -0.76 & -0.53 & -0.61 & -0.74 & -0.70 & -0.61 & -0.61 & 0.08 \\
\hline $\begin{array}{l}\text { Hta1; } \\
\text { Hta2 }\end{array}$ & \begin{tabular}{|l|} 
P04911; \\
P04912
\end{tabular} & -0.64 & -0.61 & -0.59 & -0.73 & -0.62 & -0.66 & -0.61 & -0.64 & -0.58 & -0.62 & 0.04 \\
\hline Gcv3 & P39726 & $\mathrm{NaN}$ & -0.48 & -0.62 & -0.54 & -0.80 & -0.93 & -0.73 & -0.62 & -0.42 & -0.62 & 0.17 \\
\hline Mia40 & P36046 & -0.52 & -0.56 & -0.52 & -0.61 & -0.76 & -0.63 & -0.66 & -0.81 & -0.62 & -0.62 & 0.10 \\
\hline Tuf1 & P02992 & -0.70 & -0.54 & -0.62 & -0.71 & -0.88 & -0.52 & -0.65 & -0.62 & -0.51 & -0.62 & 0.12 \\
\hline $\begin{array}{l}\text { Htb1; } \\
\text { Htb2 }\end{array}$ & $\begin{array}{l}\text { P02293; } \\
\text { P02294 }\end{array}$ & -0.58 & -0.53 & -0.60 & -0.62 & -0.57 & -0.70 & -0.77 & -0.71 & -0.63 & -0.62 & 0.08 \\
\hline Isc1 & P40015 & $\mathrm{NaN}$ & $\mathrm{NaN}$ & $\mathrm{NaN}$ & $\mathrm{aN}$ & $\mathrm{NaN}$ & -0.63 & -0.62 & $\mathrm{NaN}$ & $\mathrm{NaN}$ & -0.63 & 0.01 \\
\hline Mrp13 & P12686 & -0.24 & -0.46 & -0.63 & $\mathrm{NaN}$ & -0.75 & $\mathrm{NaN}$ & -0.71 & -0.83 & -0.43 & -0.63 & 0.21 \\
\hline Rpp2a & P05319 & -0.61 & -0.58 & -0.55 & -0.53 & -0.64 & -0.74 & -0.63 & -0.65 & -0.69 & -0.63 & 0.07 \\
\hline Tsa1 & P34760 & -0.56 & -0.64 & -0.64 & -0.64 & -0.69 & -0.59 & -0.62 & -0.68 & -0.62 & -0.64 & 0.04 \\
\hline Gas3 & Q03655 & -0.67 & -0.64 & -0.48 & -0.34 & -0.28 & -0.96 & -0.60 & -0.90 & -0.99 & -0.64 & 0.26 \\
\hline Hht1 & P61830 & -0.81 & -0.66 & -0.64 & -0.72 & -0.61 & -0.69 & -0.59 & -0.71 & -0.57 & -0.66 & 0.08 \\
\hline Tim9 & 074700 & -0.66 & -0.67 & -0.67 & -0.65 & -0.53 & -0.57 & -0.72 & -0.22 & -0.74 & -0.66 & 0.16 \\
\hline Ggc1 & P38988 & -0.78 & -0.72 & -0.70 & -0.67 & -0.67 & -0.64 & -0.54 & -0.55 & -0.68 & -0.67 & 0.08 \\
\hline Pmc1 & P38929 & -0.36 & $\mathrm{NaN}$ & -0.67 & -0.28 & -0.10 & $\mathrm{NaN}$ & -1.10 & -1.21 & -0.68 & -0.67 & 0.42 \\
\hline Mcd4 & P36051 & -0.76 & -0.61 & -0.80 & -0.66 & -0.49 & -0.18 & -0.69 & -0.85 & -0.67 & -0.67 & 0.20 \\
\hline Tim10 & P87108 & -0.76 & -0.66 & -0.62 & -0.41 & -0.68 & -0.69 & -0.64 & -0.68 & -0.80 & -0.68 & 0.11 \\
\hline Hho1 & P53551 & -0.69 & -0.61 & -0.89 & -0.91 & $\mathrm{NaN}$ & -0.62 & $\mathrm{NaN}$ & $\mathrm{NaN}$ & $\mathrm{NaN}$ & -0.69 & 0.15 \\
\hline Pdi1 & \begin{tabular}{|l|} 
P17967 \\
\end{tabular} & -0.67 & -0.69 & -0.73 & -0.64 & -0.70 & -0.62 & -0.67 & -0.70 & -0.70 & -0.69 & 0.03 \\
\hline Adk1 & P07170 & -0.70 & -0.60 & -0.67 & -0.70 & -0.71 & -0.70 & -0.71 & -0.66 & -0.68 & -0.70 & 0.03 \\
\hline Hem1 & P09950 & -0.76 & -0.89 & -0.64 & -0.43 & -0.70 & -0.67 & -0.83 & -0.71 & -0.55 & -0.70 & 0.14 \\
\hline Mrp1 & P10662 & -0.74 & -0.52 & -0.29 & -0.29 & -0.82 & -0.67 & -0.89 & -0.90 & $\mathrm{NaN}$ & -0.71 & 0.25 \\
\hline Lys20 & P48570 & -0.94 & -0.97 & -0.87 & -0.66 & -0.42 & -0.71 & -0.30 & -0.82 & -0.66 & -0.71 & 0.23 \\
\hline Sap190 & P36123 & -0.73 & -0.71 & -0.42 & $\mathrm{NaN}$ & 0.08 & $\mathrm{NaN}$ & -0.82 & -0.93 & -0.59 & -0.71 & 0.34 \\
\hline Ynl208w & \begin{tabular}{|l|} 
P40159 \\
\end{tabular} & -0.71 & -0.83 & -0.71 & -0.66 & -0.81 & -0.94 & -0.69 & -0.79 & -0.44 & -0.71 & 0.14 \\
\hline Nce102 & Q12207 & -0.55 & -0.75 & -0.62 & -0.72 & -1.36 & -0.55 & -0.88 & -0.97 & -0.46 & -0.72 & 0.28 \\
\hline Glt1 & Q12680 & -0.72 & -0.73 & -0.78 & -0.64 & -0.71 & -0.83 & -0.79 & -0.79 & -0.72 & -0.73 & 0.06 \\
\hline Scw4 & P53334 & -0.49 & -0.64 & -0.75 & -0.81 & -0.57 & -0.83 & -1.17 & -0.94 & -0.37 & -0.75 & 0.24 \\
\hline Ydl124w & Q07551 & -0.48 & -0.52 & -0.79 & -0.75 & -0.96 & -0.45 & -0.85 & -0.90 & -0.57 & -0.75 & 0.19 \\
\hline Gcv2 & P49095 & -0.78 & -0.75 & -0.86 & -1.05 & -0.58 & -0.26 & -0.73 & -0.77 & -0.47 & -0.75 & 0.23 \\
\hline$A \times 12$ & P38928 & $\mathrm{NaN}$ & $\mathrm{NaN}$ & -0.66 & $\mathrm{NaN}$ & $\mathrm{NaN}$ & $\mathrm{NaN}$ & -0.77 & -0.79 & -0.74 & -0.75 & 0.06 \\
\hline Ynl134c & P53912 & -0.60 & -0.76 & -0.66 & -0.57 & -0.86 & -0.78 & -0.92 & -0.78 & -0.53 & -0.76 & 0.14 \\
\hline Uth1 & P36135 & -0.65 & -0.66 & -1.42 & -0.87 & $\mathrm{NaN}$ & -1.03 & -0.55 & -0.95 & -0.32 & -0.76 & 0.34 \\
\hline Ycf1 & P39109 & -0.55 & -0.38 & -0.64 & -0.77 & -0.82 & -1.27 & -0.81 & -0.83 & -0.61 & -0.77 & 0.25 \\
\hline Yjr098c & P47139 & -0.61 & -0.73 & -0.67 & -0.92 & -0.80 & $\mathrm{NaN}$ & $\mathrm{NaN}$ & -0.86 & -0.78 & -0.78 & 0.11 \\
\hline
\end{tabular}


Supplementary Table 4. Continued 3.

\begin{tabular}{|c|c|c|c|c|c|c|c|c|c|c|c|c|}
\hline \multirow{2}{*}{ proteins } & \multirow{2}{*}{$\begin{array}{l}\text { protein } \\
\text { IDs }\end{array}$} & \multicolumn{9}{|c|}{$\operatorname{asc1/ASC1}$} & \multirow[b]{2}{*}{$\varnothing$} & \multirow[b]{2}{*}{ SD } \\
\hline & & 1 & 2 & 3 & 4 & 5 & 6 & 7 & 8 & 13 & & \\
\hline Ybr085c-A & 043137 & -0.63 & -0.79 & -0.83 & -1.15 & -1.24 & -0.71 & -0.92 & -0.68 & -0.47 & -0.79 & 0.25 \\
\hline Kar2 & P16474 & -0.72 & -0.81 & -0.80 & -0.79 & -0.75 & -0.63 & -0.76 & -0.79 & -0.80 & -0.79 & 0.06 \\
\hline Nhp6a & P11632 & $\mathrm{NaN}$ & -0.79 & -0.64 & -0.79 & $\mathrm{NaN}$ & $\mathrm{NaN}$ & -0.94 & $\mathrm{NaN}$ & $\mathrm{NaN}$ & -0.79 & 0.12 \\
\hline Taf5 & P38129 & $\mathrm{NaN}$ & $\mathrm{NaN}$ & -1.00 & -0.40 & -0.64 & -0.69 & -1.62 & -0.90 & $\mathrm{NaN}$ & -0.79 & 0.42 \\
\hline Yhm2 & Q04013 & -0.84 & -0.73 & -0.84 & -0.75 & -0.72 & -0.85 & -0.80 & -0.88 & -0.72 & -0.80 & 0.06 \\
\hline Dic1 & Q06143 & $\mathrm{NaN}$ & -1.04 & -0.80 & -1.16 & -0.84 & $\mathrm{NaN}$ & -0.46 & -0.33 & -0.63 & -0.80 & 0.30 \\
\hline $\mathrm{Hxt1}$ & P32465 & -0.74 & -0.58 & -0.68 & -0.81 & -0.95 & -1.02 & -1.04 & -1.26 & -0.57 & -0.81 & 0.23 \\
\hline Agp1 & P25376 & $\mathrm{NaN}$ & -0.84 & -1.07 & -0.83 & -0.81 & $\mathrm{NaN}$ & -0.48 & -0.48 & -0.81 & -0.81 & 0.21 \\
\hline Hor2 & P40106 & -0.83 & -0.72 & -0.86 & -1.10 & -0.69 & $\mathrm{NaN}$ & $\mathrm{NaN}$ & $\mathrm{NaN}$ & $\mathrm{NaN}$ & -0.83 & 0.16 \\
\hline Psa1 & \begin{tabular}{|l|} 
P41940 \\
\end{tabular} & -0.75 & -0.77 & -0.86 & -0.83 & -0.88 & -0.86 & -0.86 & -0.96 & -0.84 & -0.86 & 0.06 \\
\hline Var1 & P02381 & -1.00 & $\mathrm{NaN}$ & $\mathrm{NaN}$ & -0.71 & $\mathrm{NaN}$ & $\mathrm{NaN}$ & -0.87 & $\mathrm{NaN}$ & $\mathrm{NaN}$ & -0.87 & 0.14 \\
\hline Gph1 & P06738 & -0.82 & -0.87 & -1.11 & -1.51 & -1.19 & 0.01 & -1.07 & -0.75 & -0.21 & -0.87 & 0.48 \\
\hline Ura4 & P20051 & -0.74 & -0.90 & -1.07 & -0.87 & -0.64 & -1.00 & -1.06 & -0.80 & -1.04 & -0.90 & 0.15 \\
\hline Pbi2 & Р0СT04 & -0.86 & -0.64 & -1.11 & -1.22 & -1.35 & -0.52 & -0.90 & -1.12 & -0.68 & -0.90 & 0.28 \\
\hline Rhr2 & P41277 & -0.90 & -0.99 & -0.88 & -0.91 & -0.93 & -1.22 & -0.99 & -0.91 & -0.89 & -0.91 & 0.11 \\
\hline$H x+6^{6}$ & P39003 ${ }^{6}$ & -0.84 & -0.94 & -1.21 & -1.09 & -1.41 & 0.21 & -1.07 & -0.91 & -0.28 & -0.94 & 0.50 \\
\hline Tos 1 & P38288 & -1.03 & -0.81 & -0.99 & -1.03 & $\mathrm{NaN}$ & -0.38 & -1.06 & -0.95 & -0.69 & -0.97 & 0.23 \\
\hline Ctp1 & \begin{tabular}{|l|} 
P38152 \\
\end{tabular} & -1.32 & -0.97 & -0.97 & -0.71 & -0.81 & -1.15 & -0.70 & -1.04 & -0.57 & -0.97 & 0.24 \\
\hline Exg1 & P23776 & -0.73 & -0.90 & -0.84 & -1.12 & -1.04 & -1.00 & -1.25 & -1.16 & -0.75 & -1.00 & 0.18 \\
\hline Gsc2 & P40989 & -1.48 & -1.09 & -1.12 & -0.92 & -0.90 & -0.13 & -1.05 & -1.00 & -0.55 & -1.00 & 0.38 \\
\hline Tma17 & Q12513 & $\mathrm{NaN}$ & -1.22 & -0.98 & $\mathrm{NaN}$ & $\mathrm{NaN}$ & $\mathrm{NaN}$ & $\mathrm{NaN}$ & $\mathrm{NaN}$ & -1.01 & -1.01 & 0.13 \\
\hline Ftr1 & \begin{tabular}{|l|} 
P40088 \\
\end{tabular} & -0.54 & 0.01 & -0.97 & -1.18 & $\mathrm{NaN}$ & $\mathrm{NaN}$ & -1.19 & $\mathrm{NaN}$ & -1.11 & -1.04 & 0.48 \\
\hline Cpr1 & P14832 & -1.00 & -0.94 & -0.99 & -1.07 & -1.19 & -1.08 & -1.16 & -1.12 & -0.96 & -1.07 & 0.09 \\
\hline Gre2 & Q12068 & $\mathrm{NaN}$ & $\mathrm{NaN}$ & -1.37 & -1.19 & -0.95 & -0.57 & -0.87 & -1.65 & $\mathrm{NaN}$ & -1.07 & 0.39 \\
\hline Sim1 & \begin{tabular}{|l|l} 
P40472 \\
\end{tabular} & -0.56 & $\mathrm{NaN}$ & -1.41 & -1.48 & $\mathrm{NaN}$ & $\mathrm{NaN}$ & -1.13 & -1.19 & -0.85 & -1.16 & 0.35 \\
\hline Mnn1 & P39106 & -0.97 & -0.98 & -1.18 & -1.34 & -1.17 & -1.20 & -1.05 & -1.20 & -0.85 & -1.17 & 0.15 \\
\hline Pdr12 & Q02785 & -1.35 & -0.49 & -2.21 & -1.32 & -1.38 & $\mathrm{NaN}$ & -1.84 & -1.26 & -0.82 & -1.34 & 0.54 \\
\hline Ura1 & P28272 & -1.18 & -1.37 & -1.50 & -1.35 & -1.16 & -1.42 & -1.48 & -1.22 & -1.47 & -1.37 & 0.13 \\
\hline Msc1 & Q03104 & -1.44 & -0.89 & -1.67 & -0.93 & $\mathrm{NaN}$ & -0.74 & -2.13 & -1.96 & $\mathrm{NaN}$ & -1.44 & 0.55 \\
\hline Fet3 & \begin{tabular}{|l|} 
P38993 \\
\end{tabular} & -1.26 & -1.06 & -0.82 & -1.17 & -1.45 & -2.12 & -1.59 & -1.90 & -1.65 & -1.45 & 0.41 \\
\hline Cpa1 & \begin{tabular}{|l|} 
P07258 \\
\end{tabular} & -1.87 & $\mathrm{NaN}$ & -1.79 & -1.50 & $\mathrm{NaN}$ & $\mathrm{NaN}$ & $\mathrm{NaN}$ & $\mathrm{NaN}$ & -1.78 & -1.78 & 0.16 \\
\hline Rib4 & P50861 & -1.64 & -1.94 & -1.84 & -1.72 & -1.58 & -1.81 & -1.98 & -1.64 & -2.03 & -1.81 & 0.16 \\
\hline Ynr034w-A & Q3E841 & $\mathrm{NaN}$ & -1.97 & -2.69 & $\mathrm{NaN}$ & -1.74 & $\mathrm{NaN}$ & -1.58 & $\mathrm{NaN}$ & $\mathrm{NaN}$ & -1.86 & 0.49 \\
\hline Mdh2 & P22133 & 2.03 & -2.53 & -3.29 & -1.89 & -1.34 & -1.52 & $\mathrm{NaN}$ & -1.95 & -2.14 & -1.99 & 0.61 \\
\hline Ctt1 & P06115 & 1.93 & -1.57 & -2.35 & -2.00 & -2.53 & -1.08 & -2.03 & -2.55 & -0.90 & -2.00 & 0.59 \\
\hline Ygp1 & P38616 & -2.01 & -1.14 & -3.27 & -3.16 & $\mathrm{NaN}$ & $\mathrm{NaN}$ & -1.16 & -2.17 & $\mathrm{NaN}$ & -2.09 & 0.93 \\
\hline Hsp12 & P22943 & -3.06 & -2.08 & -2.41 & -3.07 & -3.79 & -1.68 & -3.96 & $\mathrm{NaN}$ & $\mathrm{NaN}$ & -3.06 & 0.85 \\
\hline Asc1 & P38011 & -9.32 & -10.8 & -8.51 & -8.96 & -3.51 & -3.63 & -3.60 & -4.19 & -4.62 & -4.62 & 2.97 \\
\hline
\end{tabular}

${ }^{1}$ Ty1a-Pr1;Ty1a-A;Ty1a-Dr4;Ty1a-Jr2;Ty1a-NI2;P0CX58;P0CX57;O74302;P47099;Q12470

${ }^{2}$ Ty2b-C;Ty2b-Gr2;Ty2b-F;Ty2b-Dr2;P25384;P0CX64;P0CX63;Q03494

${ }^{3}$ Ty1b-Er1;Ty1b-MI2;Ty1b-Ol;Ty1b-Jr1;Ty1b-A;Q03612;Q03434;Q12273;P47098;O13527

${ }^{4}$ Ty1a-PI;Ty1a-Lr2;Ty1a-Er1;Ty1a-Dr6;Ty1a-Pr3;Ty1a-Gr1;Ty1a-MI2;Ty1a-Lr3;Ty1a-Jr1;Ty1a-Lr4;

Ty1a-Gr2;Ty1a-Ol;P0CX73;P0CX72;P0CX71;P0CX70;Q6Q5H1;Q12085;P0CX76;P0CX75;P0CX74;

P0C218;Q12485;Q92392;Q12391

${ }^{5}$ Ty2b-Gr1;Ty2b-Lr1;Ty2b-Dr1;Ty2b-Or2;Q12337;P0C2J3;Q12472;Q12501;P0C2J2

${ }^{6}$ Hxt7,P39004;Hxt10,P43581;Hxt12,P40441;Hxt13,P39924;Hxt17,P53631;Gal2,P13181 
Supplementary Table 5. SILAC-based proteome data for the comparison of the asc1DE strain and the $A S C 1$ wild-type strain.

The table lists the two proteins with a median asc1DE/ASC1 $\log _{2}$ SILAC-ratio $\geq 0.58$ and a p-value $<0.01$ (no protein with a median asc1DE/ASC1 $\log _{2}$ SILAC-ratio $\leq-0.58$ was identified). Proteins with this SILAC-ratio $>0.26$ or $<-0.26$ are not shown). The replicates are numbered 9-13 according to Figure 21. ( $\varnothing=$ median of protein SILAC-ratios; $\mathrm{SD}=$ standard deviation; gray/NaN = not a number $)$

\begin{tabular}{|c|c|c|c|c|c|c|c|c|}
\hline \multirow{2}{*}{ proteins } & \multirow{2}{*}{$\begin{array}{l}\text { protein } \\
\text { IDs }\end{array}$} & \multicolumn{5}{|c|}{ asc1DE/ASC1 } & \multirow[b]{2}{*}{$\varnothing$} & \multirow[b]{2}{*}{ SD } \\
\hline & & 9 & 10 & 11 & 12 & 13 & & \\
\hline Ty1a $a^{1}$ & P0CX73 1 & 0.72 & 0.63 & $\mathrm{NaN}$ & $\mathrm{NaN}$ & 0.73 & 0.72 & 0.05 \\
\hline Nup57 & P48837 & 0.35 & $\mathrm{NaN}$ & 0.60 & 0.58 & 0.58 & 0.58 & 0.12 \\
\hline
\end{tabular}

*Ty1a-PI;Ty1a-Lr2;Ty1a-Er1;Ty1a-Dr6;Ty1a-Pr3;Ty1a-Gr1;Ty1a-MI2;Ty1a-Lr3;Ty1a-Jr1; Ty1a-Lr4;Ty1a-Gr2;Ty1a-OI

P0CX73;P0CX72;P0CX71;P0CX70;Q6Q5H1;Q12085;P0CX76;P0CX75;P0CX74;P0C2I8;Q12485; Q92392;Q12391

Supplementary Table 6. SILAC-based proteome data for the comparison of the asc $1^{\text {T143A }}$ strain and the $A S C 1$ wild-type strain.

The table lists the protein that fulfilled the criteria of the two-sample $t$-test (see Supplementary Table 2 ) and had a A-Aux $\log _{2}$ SILAC-ratio > 0.26. The replicates are numbered 1-3 and 4, 8, and 12 according to Figure 21. ( $\varnothing=$ mean of protein SILAC-ratios for A and Aux; $\mathrm{A}=\operatorname{ascl}^{\mathrm{T} 143 \mathrm{~A}} / \mathrm{ASCl}$;

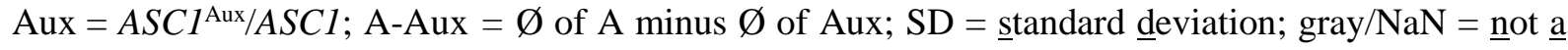
number)

\begin{tabular}{|c|c|c|c|c|c|c|c|c|c|c|c|c|}
\hline \multirow{2}{*}{ protein } & \multirow{2}{*}{$\begin{array}{l}\text { protein } \\
\text { ID }\end{array}$} & \multicolumn{3}{|c|}{$\operatorname{asc} 1^{\mathrm{T} 143 \mathrm{~A}} / \mathrm{ASC} 1$} & \multicolumn{3}{|c|}{ ASC1Aux/ASC1 } & \multicolumn{2}{|c|}{ A } & \multicolumn{2}{|c|}{ Aux } & \multirow{2}{*}{$\begin{array}{c}\text { A - } \\
\text { Aux }\end{array}$} \\
\hline & & 1 & 2 & 3 & 4 & 8 & 12 & $\varnothing$ & SD & $\varnothing$ & SD & \\
\hline Cox20 & Q04935 & $\mathrm{NaN}$ & 0.19 & 0.22 & $\mathrm{NaN}$ & -0.15 & -0.15 & 0.20 & 0.02 & -0.15 & 0.00 & 0.35 \\
\hline
\end{tabular}

Supplementary Table 7. SILAC-based proteome data for the comparison of the asc ${ }^{\text {T143E }}{ }^{\text {strain }}$ and the $A S C 1$ wild-type strain.

The table lists the proteins that fulfilled the criteria of the two-sample $t$-test (see Supplementary Table 2 ) and had a E-Aux $\log _{2}$ SILAC-ratio $<-0.26$ or $>0.26$. The replicates are numbered 5-6 and 4, 8, and 12 according to Figure 21. ( $\varnothing=$ mean of protein SILAC-ratios for $\mathrm{E}$ and Aux; $\mathrm{E}=\operatorname{ascl}^{\mathrm{T} 143 \mathrm{E}} / \mathrm{ASCl}$; Aux $=A S C 1^{\text {Aux }} / A S C 1 ; \mathrm{E}-\mathrm{Aux}=\varnothing$ of $\mathrm{E}$ minus $\varnothing$ of Aux; $\mathrm{SD}=$ standard deviation; gray $/ \mathrm{NaN}=\underline{\text { not }}$ a number)

\begin{tabular}{|c|c|c|c|c|c|c|c|c|c|c|c|c|}
\hline \multirow{2}{*}{ proteins } & \multirow{2}{*}{$\begin{array}{l}\text { protein } \\
\text { IDs }\end{array}$} & \multicolumn{3}{|c|}{ asc1 1143E/ASC1 } & \multicolumn{3}{|c|}{$A S C 1^{\text {Aux }} / A S C 1$} & \multicolumn{2}{|c|}{$\mathrm{E}$} & \multicolumn{2}{|c|}{ Aux } & \multirow{2}{*}{$\begin{array}{l}\text { E - } \\
\text { Aux }\end{array}$} \\
\hline & & 5 & 6 & 7 & 4 & 8 & 12 & $\varnothing$ & $\mathrm{SD}$ & $\varnothing$ & SD & \\
\hline Rcl1 & 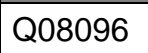 & $\mathrm{NaN}$ & 0.07 & 0.04 & -0.26 & $\mathrm{NaN}$ & -0.29 & 0.06 & 0.02 & -0.28 & 0.02 & 0.34 \\
\hline Aha1 & & 0.11 & 0.09 & 0.10 & -0.18 & -0.20 & -0.11 & 0.10 & 0.01 & -0.17 & 0.05 & 0.26 \\
\hline Efg1 & 5 & -0.02 & -0.02 & $\mathrm{NaN}$ & 0.24 & 0.32 & 0.24 & -0.02 & 0.00 & 0.27 & 0.05 & -0.29 \\
\hline Rvb2 & Q12464 & -0.17 & -0.12 & -0.14 & 0.18 & 0.10 & 0.18 & -0.15 & 0.02 & 0.15 & 0.05 & -0.30 \\
\hline
\end{tabular}




\section{Supplementary Table 8. SILAC-based proteome data asc ${ }^{\text {T143E }}$ DE versus ASC1 wild-type.}

The table lists the proteins that fulfilled the criteria of the two-sample $t$-test (see Supplementary Table 2) and had a mean ${ }^{E}$ DE-Aux $\log _{2}$ SILAC-ratio $\leq-0.58$ or $\geq 0.58$. Proteins with this SILAC-ratio $<-0.26$ or $>0.26$ are not shown. The replicates are numbered $9-11$ and 4,8 , and 12 according to Figure 21. $\left(\varnothing=\right.$ mean of protein SILAC-ratios for ${ }^{\mathrm{E}} \mathrm{DE}$ and Aux; ${ }^{\mathrm{E}} \mathrm{DE}=$ asc ${ }^{\mathrm{T} 143 \mathrm{E}} \mathrm{DE} / \mathrm{ASCl}$; Aux $=$

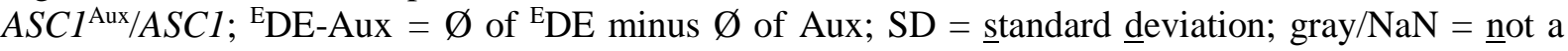
number)

\begin{tabular}{|c|c|c|c|c|c|c|c|c|c|c|c|c|}
\hline \multirow{2}{*}{ proteins } & \multirow{2}{*}{$\begin{array}{l}\text { protein } \\
\text { IDs }\end{array}$} & \multicolumn{3}{|c|}{ asc1 1143EDE/ASC1 } & \multicolumn{3}{|c|}{ ASC1Aux/ASC1 } & \multicolumn{2}{|c|}{ EDE } & \multicolumn{2}{|c|}{ Aux } & \multirow{2}{*}{$\begin{array}{l}{ }^{E D E} \\
\text {-Aux }\end{array}$} \\
\hline & & 9 & 10 & 11 & 4 & 8 & 12 & $\varnothing$ & SD & $\varnothing$ & $\mathrm{SD}$ & \\
\hline ce103 & 53615 & 0.57 & 0.85 & 0.81 & -0.46 & -0.43 & -0.13 & 0.74 & 0.15 & -0.34 & 0.18 & 1.08 \\
\hline Ty $1 b^{1}$ & $03612^{1}$ & 0.82 & 1.14 & 1.12 & -0.11 & 0.13 & -0.15 & 1.03 & 0.18 & -0.05 & 0.15 & 1.07 \\
\hline Yhb1 & P39676 & 0.88 & 0.73 & 0.68 & -0.05 & -0.29 & -0.35 & 0.76 & 0.10 & -0.23 & 0.16 & 0.99 \\
\hline Hbn1 & 66VH4 & 0.65 & 0.64 & 0.35 & -0.16 & -0.39 & -0.55 & 0.55 & 0.17 & -0.37 & 0.19 & 0.91 \\
\hline Hsp82 & P02829 & 0.84 & 0.98 & 0.85 & 0.10 & -0.13 & 0.12 & 0.89 & 0.08 & 0.03 & 0.14 & 0.86 \\
\hline Aap1 & 98 & 0.62 & 0.68 & 0.62 & -0.02 & -0.07 & -0.13 & 0.64 & 0.03 & -0.07 & 0.06 & 0.71 \\
\hline Ygl039w & P53183 & 0.50 & 0.18 & 0.58 & -0.30 & -0.16 & -0.28 & 0.42 & 0.21 & -0.25 & 0.07 & 0.67 \\
\hline Car1 & 12 & 0.53 & 0.73 & 0.68 & 0.07 & 0.06 & -0.14 & 0.65 & 0.10 & 0.00 & 0.12 & 0.65 \\
\hline Ych1 & P42937 & 0.44 & 0.35 & 0.43 & -0.43 & -0.19 & -0.01 & 0.41 & 0.05 & -0.21 & 0.21 & 0.62 \\
\hline Gly1 & 3 & 0.49 & 0.62 & 0.74 & 0.02 & 0.16 & -0.12 & 0.62 & 0.12 & 0.02 & 0.14 & 0.60 \\
\hline Cpa2 & P03965 & 0.49 & 0.57 & 0.58 & -0.10 & 0.06 & -0.10 & 0.55 & 0.05 & -0.05 & 0.09 & 0.59 \\
\hline Sui1 & P32911 & 0.62 & 0.66 & 0.67 & 0.03 & 0.18 & -0.04 & 0.65 & 0.03 & 0.06 & 0.11 & 0.59 \\
\hline Arg1 & P22768 & 0.92 & 0.83 & 0.97 & 0.26 & 0.47 & 0.23 & 0.91 & 0.07 & 0.32 & 0.13 & 0.59 \\
\hline Scw10 & Q04951 & -1.00 & -0.77 & -0.95 & -0.34 & -0.30 & 0.05 & -0.91 & 0.12 & -0.20 & 0.21 & -0.71 \\
\hline Gsc2 & P40989 & -0.74 & -0.83 & -0.91 & -0.07 & -0.26 & 0.10 & -0.83 & 0.08 & -0.08 & 0.18 & -0.75 \\
\hline Mnn1 & P39106 & -0.80 & -0.62 & -0.73 & 0.14 & -0.01 & 0.12 & -0.72 & 0.09 & 0.08 & 0.08 & -0.80 \\
\hline Ura4 & P20051 & -0.80 & -0.87 & -0.86 & 0.02 & -0.02 & -0.04 & -0.84 & 0.04 & -0.01 & 0.03 & -0.83 \\
\hline Ura1 & P28272 & -0.80 & -0.90 & -0.94 & 0.18 & -0.04 & 0.16 & -0.88 & 0.07 & 0.10 & 0.12 & -0.98 \\
\hline Rib4 & P50861 & -1.04 & -1.12 & -1.03 & 0.10 & 0.04 & -0.04 & -1.07 & 0.04 & 0.03 & 0.07 & -1.10 \\
\hline Arp7 & Q12406 & -1.65 & -1.45 & $\mathrm{NaN}$ & 0.17 & 0.15 & -0.06 & -1.55 & 0.14 & 0.09 & 0.13 & 1.64 \\
\hline
\end{tabular}

${ }^{1}$ Ty1b-Er1;Ty1b-MI2;Ty1b-OI;Ty1b-Jr1;Ty1b-A

Q03612;Q03434;Q12273;P47098;O13527 
Supplementary Table 9. SILAC-based phospho-proteome data for the comparison of the asc $1^{-}$ strain and the $A S C 1$ wild-type strain.

The table lists the proteins with a proteome-corrected phospho-peptide $\log _{2}$ asc1-/ASC1 SILAC-ratio (diff. phos-prot) $>0.26$ or $<-0.26$ and a p-value $<0.01$ in the two-sample $t$-test (Supplementary Table 2). The replicates are numbered 1-8, and 13 according to Figure 21. ( $\varnothing=$ median; SD $=\underline{\text { standard }}$ deviation; difference (diff.) phos-prot $=\varnothing$ phospho-peptide (phos) minus $\varnothing$ protein ratio (prot); positon $=$ phosphorylated amino acid residue, printed in italic = site was previously unknown; loc. prob. $=$ localization probability; gray $/ \mathrm{NaN}=$ not a number; multiplicity $=$ number of phospho-sites per peptide)

\begin{tabular}{|c|c|c|c|c|c|c|c|c|c|c|c|}
\hline \multirow{2}{*}{ proteins } & \multirow{2}{*}{$\begin{array}{l}\text { protein } \\
\text { IDs }\end{array}$} & \multirow{2}{*}{ 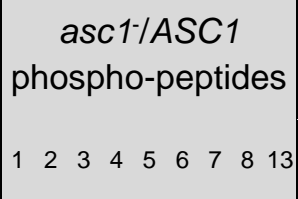 } & \multirow{2}{*}{ 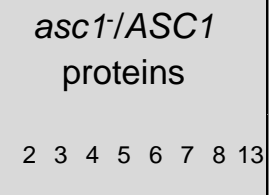 } & \multicolumn{2}{|c|}{$\begin{array}{c}\text { phos- } \\
\text { peptides }\end{array}$} & \multicolumn{2}{|c|}{ proteins } & \multirow{2}{*}{$\begin{array}{l}\text { diff. } \\
\text { phos- } \\
\text { prot }\end{array}$} & \multirow{2}{*}{ position } & \multirow{2}{*}{ 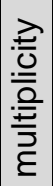 } & \multirow{2}{*}{$\begin{array}{l}\text { 음 } \\
\text { 음 } \\
\text { 으 }\end{array}$} \\
\hline & & & & $\varnothing$ & SD & $\varnothing$ & SD & & & & \\
\hline Tpi1 & P00942 & & & 1.19 & 0.20 & -0.28 & 0.04 & 1.47 & S 215 & 1 & 1.00 \\
\hline Csr1 & Q06705 & & & 1.31 & 0.22 & -0.14 & 0.17 & 1.45 & S 2 & 1 & 1.00 \\
\hline Pre8 & P23639 & & & 1.44 & 0.17 & 0.28 & 0.04 & 1.16 & S 15 & 1 & 1.00 \\
\hline Rpn7 & Q06103 & & & 1.27 & 0.20 & 0.15 & 0.05 & 1.11 & $S 196$ & 1 & 1.00 \\
\hline Isc1 & P40015 & & & 0.33 & 0.01 & -0.63 & 0.01 & 0.95 & T 361 & 1 & 1.00 \\
\hline $\begin{array}{l}\text { Rpl12a; } \\
\text { Rpl12b }\end{array}$ & $\begin{array}{l}\text { P0CX53; } \\
\text { P0CX54 }\end{array}$ & & & 0.74 & 0.34 & -0.19 & 0.05 & 0.93 & S 38 & 1 & 1.00 \\
\hline Ste20 & Q03497 & & & 0.56 & 0.47 & -0.34 & 0.33 & 0.90 & Т 572 & 1 & 0.61 \\
\hline Pin4 & P34217 & & & 0.69 & 0.25 & -0.17 & 0.15 & 0.87 & S 194 & 1 & 1.00 \\
\hline Ste20 & Q03497 & & & 0.48 & 0.17 & -0.34 & 0.33 & 0.82 & S 169 & 1 & 1.00 \\
\hline Imh1 & Q06704 & & & 0.41 & 0.18 & -0.40 & 0.18 & 0.81 & T 304 & 1 & 0.65 \\
\hline Acm1 & Q08981 & & & 0.60 & 0.17 & -0.20 & 0.08 & 0.80 & S 48 & 1 & 1.00 \\
\hline Imh1 & Q06704 & & & 0.32 & 0.08 & -0.40 & 0.18 & 0.72 & S 308 & 1 & 0.98 \\
\hline Smi1 & P32566 & & & 0.49 & 0.53 & -0.22 & 0.12 & 0.72 & S 389 & 1 & 0.92 \\
\hline Iml2 & P47031 & & & 0.39 & 0.26 & -0.31 & 0.18 & 0.70 & S 7 & 1 & 1.00 \\
\hline Kri1 & P42846 & & & 0.68 & 0.01 & 0.00 & 0.21 & 0.68 & Y 482 & 1 & 0.63 \\
\hline Rlp7 & P40693 & & & 0.73 & 0.23 & 0.06 & 0.12 & 0.67 & T 120 & 1 & 1.00 \\
\hline Rpn1 & P38764 & & & 0.83 & 0.18 & 0.16 & 0.04 & 0.67 & S 19 & 1 & 1.00 \\
\hline Tif1 & P10081 & & & 0.68 & 0.24 & 0.01 & 0.03 & 0.67 & S 2 & 1 & 1.00 \\
\hline Acc1 & Q00955 & & & 0.11 & 0.22 & -0.55 & 0.09 & 0.66 & 59 & 1 & 0.87 \\
\hline Fyv8 & P46949 & & & 0.68 & 0.01 & 0.03 & 0.11 & 0.65 & S 441 & 1 & 0.99 \\
\hline Rpo21 & P04050 & & & 0.42 & 0.09 & -0.22 & 0.08 & 0.64 & T 1471 & 1 & 0.93 \\
\hline Imh1 & Q06704 & & & 0.24 & 0.14 & -0.40 & 0.18 & 0.64 & S 827 & 1 & 0.52 \\
\hline Ste20 & Q03497 & & & 0.30 & 0.20 & -0.34 & 0.33 & 0.63 & S 502 & 1 & 1.00 \\
\hline Myo3 & P36006 & & & 0.86 & 0.12 & 0.24 & 0.46 & 0.62 & S 357 & 1 & 1.00 \\
\hline Pda1 & P16387 & & & 0.51 & 0.28 & -0.11 & 0.05 & 0.62 & S 313 & 1 & 1.00 \\
\hline Pdr16 & P53860 & & & 0.50 & 0.14 & -0.11 & 0.12 & 0.61 & S 349 & 2 & 0.69 \\
\hline Pdr16 & P53860 & & & 0.50 & 0.18 & -0.11 & 0.12 & 0.61 & S 346 & 2 & 0.97 \\
\hline Leu1 & \begin{tabular}{|l|l|} 
P07264 \\
\end{tabular} & & & 0.77 & 0.24 & 0.21 & 0.05 & 0.56 & T 494 & 2 & 0.76 \\
\hline Sec3 & P33332 & & & 0.35 & 0.20 & -0.22 & 0.11 & 0.56 & S 256 & 1 & 1.00 \\
\hline Yak1 & P14680 & & & 0.29 & 0.40 & -0.26 & 0.09 & 0.55 & T 288 & 1 & 1.00 \\
\hline Pdr16 & P53860 & & & 0.43 & 0.13 & -0.11 & 0.12 & 0.55 & S 346 & 1 & 0.97 \\
\hline Hrb1 & P38922 & & & 0.37 & 0.07 & -0.18 & 0.19 & 0.54 & S 338 & 1 & 0.89 \\
\hline Crp1 & P38845 & & & 0.76 & 0.24 & 0.23 & 0.13 & 0.53 & S 440 & 1 & 0.96 \\
\hline Hrb1 & P38922 & & & 0.33 & 0.26 & -0.18 & 0.19 & 0.50 & S 343 & 1 & 0.62 \\
\hline Yef3 & P16521 & & & 0.52 & 0.28 & 0.02 & 0.04 & 0.50 & S 642 & 1 & 0.91 \\
\hline Rcn2 & Q12044 & & & 1.42 & 0.11 & 0.92 & 0.08 & 0.50 & S 104 & 1 & 0.76 \\
\hline Kri1 & P42846 & & & 0.49 & 0.09 & 0.00 & 0.21 & 0.49 & S 486 & 1 & 1.00 \\
\hline Ren2 & Q12044 & & & 1.41 & 0.15 & 0.92 & 0.08 & 0.48 & S 160 & 1 & 0.99 \\
\hline Cmd1 & \begin{tabular}{|l|} 
P06787 \\
\end{tabular} & & & 0.83 & 0.46 & 0.35 & 0.08 & 0.48 & S 82 & 1 & 1.00 \\
\hline
\end{tabular}


Supplementary Table 9. Continued 1.

\begin{tabular}{|c|c|c|c|c|c|c|c|c|c|c|c|}
\hline proteins & $\begin{array}{l}\text { protein } \\
\text { IDs }\end{array}$ & 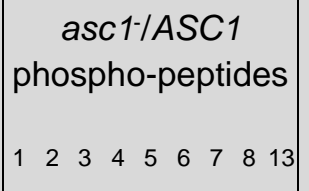 & $\begin{array}{c}\text { asc1-/ASC1 } \\
\text { proteins } \\
12345667813\end{array}$ & $\begin{array}{l}\text { phc } \\
\text { pepti } \\
\varnothing\end{array}$ & $\begin{array}{l}\text { os- } \\
\text { ides } \\
\text { SD }\end{array}$ & prote & SD & $\begin{array}{l}\text { diff. } \\
\text { phos- } \\
\text { prot }\end{array}$ & position & 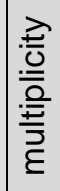 & $\begin{array}{l}\text { 음 } \\
\text { 흠 } \\
\text { 으 }\end{array}$ \\
\hline Gea2 & P39993 & & & 0.07 & 0.22 & -0.41 & 0.14 & 0.48 & S 284 & 1 & 1.00 \\
\hline Pho13 & \begin{tabular}{|l|} 
P19881 \\
\end{tabular} & & & 0.78 & 0.25 & 0.31 & 0.11 & 0.47 & T 198 & 1 & 0.93 \\
\hline Rcn2 & Q12044 & & & 1.39 & 0.30 & 0.92 & 0.08 & 0.47 & T 105 & 1 & 0.89 \\
\hline Akr1 & P39010 & & & 0.42 & 0.21 & -0.04 & 0.16 & 0.46 & S 51 & 1 & 1.00 \\
\hline Bcp1 & Q06338 & & & 0.76 & 0.22 & 0.30 & 0.10 & 0.46 & T 205 & 1 & 1.00 \\
\hline Sla2 & P33338 & & & 0.76 & 0.33 & 0.30 & 0.05 & 0.46 & T 294 & 2 & 0.87 \\
\hline Ren2 & Q12044 & & & 1.38 & 0.14 & 0.92 & 0.08 & 0.46 & S 102 & 1 & 0.80 \\
\hline Erb1 & Q04660 & & & 0.55 & 0.32 & 0.09 & 0.06 & 0.46 & S 418 & 1 & 1.00 \\
\hline Pho13 & P19881 & & & 0.76 & 0.23 & 0.31 & 0.11 & 0.45 & S 197 & 1 & 0.94 \\
\hline $\begin{array}{l}\text { Rps0a; } \\
\text { Rps0b }\end{array}$ & $\begin{array}{l}\text { P32905; } \\
\text { P46654 }\end{array}$ & & & 0.32 & 0.23 & -0.13 & 0.10 & 0.45 & $T$ 6;6 & 1 & 0.60 \\
\hline Rpg1 & P38249 & & & 0.59 & 0.13 & 0.14 & 0.04 & 0.44 & S 508 & 1 & 1.00 \\
\hline Nop12 & Q08208 & & & 0.49 & 0.24 & 0.05 & 0.13 & 0.44 & S 70 & 1 & 0.97 \\
\hline Gly1 & P37303 & & & 1.27 & 0.30 & 0.85 & 0.09 & 0.42 & S 367 & 1 & 0.79 \\
\hline Gly1 & \begin{tabular}{|l|} 
P37303 \\
\end{tabular} & & & 1.27 & 0.21 & 0.85 & 0.09 & 0.42 & S 369 & 1 & 1.00 \\
\hline Spa2 & P23201 & & & 0.42 & 0.24 & 0.01 & 0.12 & 0.42 & S 1080 & 1 & 1.00 \\
\hline Sec3 & \begin{tabular}{|l|} 
P33332 \\
\end{tabular} & & & 0.19 & 0.12 & -0.22 & 0.11 & 0.41 & S 254 & 1 & 0.95 \\
\hline Ski2 & P35207 & & & 0.87 & 0.09 & 0.46 & 0.13 & 0.41 & $S 2$ & 1 & 1.00 \\
\hline Tif5 & P38431 & & & 0.45 & 0.12 & 0.05 & 0.12 & 0.40 & S 184 & 1 & 0.70 \\
\hline Leo1 & P38439 & & & 0.33 & 0.15 & -0.08 & 0.12 & 0.40 & S 132 & 1 & 1.00 \\
\hline Shm2 & P37291 & & & 0.93 & 0.17 & 0.54 & 0.10 & 0.39 & S 26 & 1 & 1.00 \\
\hline Guk1 & P15454 & & & 0.12 & 0.34 & -0.28 & 0.05 & 0.39 & S 149 & 1 & 1.00 \\
\hline Met6 & P05694 & & & 0.25 & 0.25 & -0.14 & 0.06 & 0.39 & $S 675$ & 1 & 1.00 \\
\hline Crp1 & P38845 & & & 0.62 & 0.07 & 0.23 & 0.13 & 0.38 & S 153 & 1 & 0.66 \\
\hline Gcd6 & P32501 & & & 0.38 & 0.12 & 0.00 & 0.16 & 0.38 & S 538 & 1 & 1.00 \\
\hline Mkt1 & P40850 & & & 0.37 & 0.18 & -0.01 & 0.08 & 0.38 & S 362 & 1 & 0.97 \\
\hline Tif35 & Q04067 & & & 0.52 & 0.33 & 0.14 & 0.08 & 0.38 & S 2 & 1 & 1.00 \\
\hline $\begin{array}{l}\text { Rpl13a; } \\
\text { Rpl13b }\end{array}$ & $\begin{array}{l}\text { Q12690; } \\
\text { P40212 }\end{array}$ & & & 0.26 & 0.32 & -0.12 & 0.06 & 0.38 & T 15 & 1 & 1.00 \\
\hline Ent2 & \begin{tabular}{|l|} 
Q05785 \\
\end{tabular} & & & 0.63 & 0.15 & 0.25 & 0.28 & 0.37 & S 167 & 1 & 1.00 \\
\hline Leu1 & P07264 & & & 0.58 & 0.15 & 0.21 & 0.05 & 0.37 & S 488 & 2 & 1.00 \\
\hline Sup35 & P05453 & & & 0.61 & 0.10 & 0.24 & 0.07 & 0.36 & T 570 & 1 & 0.89 \\
\hline Not3 & P06102 & & & 0.27 & 0.19 & -0.09 & 0.14 & 0.36 & S 322 & 1 & 0.79 \\
\hline Srp14 & P38985 & & & 0.38 & 0.06 & 0.02 & 0.12 & 0.36 & S 8 & 1 & 1.00 \\
\hline Vps13 & Q07878 & & & 0.45 & 0.28 & 0.10 & 0.10 & 0.35 & S 1731 & 1 & 0.99 \\
\hline Shp1 & P34223 & & & 0.36 & 0.35 & 0.01 & 0.15 & 0.35 & S 322 & 1 & 0.92 \\
\hline Kap123 & P40069 & & & 0.25 & 0.04 & -0.11 & 0.02 & 0.35 & S 646 & 1 & 1.00 \\
\hline Sub2 & Q07478 & & & 0.11 & 0.24 & -0.24 & 0.06 & 0.35 & $S 2$ & 2 & 1.00 \\
\hline Spa2 & \begin{tabular}{|l|} 
P23201 \\
\end{tabular} & & & 0.35 & 0.42 & 0.01 & 0.12 & 0.35 & S 961 & 1 & 1.00 \\
\hline Apl5 & Q08951 & & & 0.20 & 0.12 & -0.15 & 0.16 & 0.35 & S 888 & 1 & 1.00 \\
\hline Rfa2 & P26754 & & & 0.43 & 0.17 & 0.08 & 0.15 & 0.35 & S 122 & 1 & 1.00 \\
\hline Mak5 & P38112 & & & 0.28 & 0.20 & -0.07 & 0.14 & 0.35 & S 678 & 1 & 1.00 \\
\hline Pdr5 & P33302 & & & 0.31 & 0.13 & -0.04 & 0.23 & 0.35 & S 840 & 1 & 0.87 \\
\hline Ser1 & P33330 & & & 0.86 & 0.26 & 0.52 & 0.09 & 0.35 & T 20 & 1 & 1.00 \\
\hline Smc4 & Q12267 & & & 0.24 & 0.19 & -0.10 & 0.13 & 0.34 & S 128 & 1 & 1.00 \\
\hline Kin28 & P06242 & & & 0.27 & 0.19 & -0.06 & 0.19 & 0.33 & T 162 & 1 & 0.98 \\
\hline Acc1 & Q00955 & & & -0.22 & 0.14 & -0.55 & 0.09 & 0.33 & $S 10$ & 1 & 1.00 \\
\hline
\end{tabular}


Supplementary Table 9. Continued 2.

\begin{tabular}{|c|c|c|c|c|c|c|c|c|c|c|c|}
\hline proteins & $\begin{array}{l}\text { protein } \\
\text { IDs }\end{array}$ & $\begin{array}{c}\text { asc1-/ASC1 } \\
\text { phospho-peptides } \\
\begin{array}{llllllllll}1 & 2 & 3 & 4 & 5 & 6 & 7 & 8 & 13\end{array}\end{array}$ & \begin{tabular}{|ccccccccc}
\multicolumn{2}{|c}{ asc 1 -/ASC1 } \\
proteins
\end{tabular} & $\begin{array}{l}\text { phi } \\
\text { pept } \\
\varnothing\end{array}$ & $\begin{array}{l}\text { os- } \\
\text { ides } \\
\text { SD }\end{array}$ & prot & SD & $\begin{array}{l}\text { diff. } \\
\text { phos- } \\
\text { prot }\end{array}$ & position & 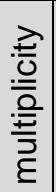 & $\begin{array}{l}\dot{0} \\
\dot{0} \\
\dot{0} \\
\underline{0}\end{array}$ \\
\hline Ssz1 & P38788 & & & 0.50 & 0.21 & 0.17 & 0.04 & 0.32 & S 480 & 1 & 1.00 \\
\hline IIs1 & P09436 & & & 0.37 & 0.14 & 0.05 & 0.04 & 0.32 & $S 4$ & 1 & 0.93 \\
\hline Prt1 & P06103 & & & 0.42 & 0.19 & 0.10 & 0.03 & 0.32 & S 61 & 1 & 1.00 \\
\hline Cdc37 & P06101 & & & 0.50 & 0.21 & 0.18 & 0.12 & 0.32 & S 17 & 2 & 1.00 \\
\hline Cdc37 & P06101 & & & 0.50 & 0.21 & 0.18 & 0.12 & 0.32 & S 14 & 2 & 1.00 \\
\hline Sec16 & \begin{tabular}{|l|l|} 
P48415 \\
\end{tabular} & & & 0.28 & 0.13 & -0.03 & 0.11 & 0.31 & T 808 & 1 & 0.77 \\
\hline Leu1 & P07264 & & & 0.52 & 0.25 & 0.21 & 0.05 & 0.31 & S 495 & 2 & 1.00 \\
\hline Pdc1 & P06169 & & & 0.13 & 0.31 & -0.17 & 0.02 & 0.30 & S 196 & 1 & 0.90 \\
\hline Nop12 & Q08208 & & & 0.35 & 0.09 & 0.05 & 0.13 & 0.30 & S 184 & 1 & 1.00 \\
\hline Vps13 & Q07878 & & & 0.40 & 0.14 & 0.10 & 0.10 & 0.30 & S 1715 & 1 & 1.00 \\
\hline Rpn1 & P38764 & & & 0.46 & 0.13 & 0.16 & 0.04 & 0.30 & S 647 & 1 & 0.81 \\
\hline Tan1 & P53072 & & & 0.52 & 0.19 & 0.22 & 0.21 & 0.30 & S 72 & 1 & 1.00 \\
\hline $\begin{array}{l}\text { Myo3; } \\
\text { Myo5 }\end{array}$ & $\begin{array}{l}\text { P36006; } \\
\text { Q04439 }\end{array}$ & & & 0.27 & 0.10 & -0.03 & 0.26 & 0.30 & S 777 & 1 & 1.00 \\
\hline Isw1 & P38144 & & & 0.26 & 0.08 & -0.04 & 0.14 & 0.29 & S 175 & 1 & 0.99 \\
\hline Sec16 & P48415 & & & 0.26 & 0.17 & -0.03 & 0.11 & 0.29 & S 607 & 1 & 1.00 \\
\hline Sup35 & P05453 & & & 0.53 & 0.20 & 0.24 & 0.07 & 0.29 & S 571 & 1 & 0.92 \\
\hline Spn1 & \begin{tabular}{|l|} 
Q06505 \\
\end{tabular} & & & 0.02 & 0.13 & -0.27 & 0.13 & 0.28 & S 89 & 1 & 1.00 \\
\hline Sec7 & P11075 & & & 0.12 & 0.02 & -0.16 & 0.09 & 0.28 & Y 1221 & 1 & 0.78 \\
\hline Sub2 & Q07478 & & & 0.04 & 0.24 & -0.24 & 0.06 & 0.28 & S 13 & 2 & 1.00 \\
\hline Nop12 & Q08208 & & & 0.32 & 0.18 & 0.05 & 0.13 & 0.28 & T 181 & 1 & 0.95 \\
\hline Vtc2 & P43585 & & & 0.57 & 0.14 & 0.30 & 0.19 & 0.27 & S 657 & 1 & 1.00 \\
\hline $\begin{array}{l}\text { Rps0a; } \\
\text { Rps0b }\end{array}$ & $\begin{array}{l}\text { P32905; } \\
\text { P46654 }\end{array}$ & & & 0.13 & 0.14 & -0.13 & 0.10 & 0.27 & S 2;2 & 1 & 0.99 \\
\hline Ser33 & P40510 & & & -0.37 & 0.29 & -0.10 & 0.12 & -0.27 & S 29 & 2 & 0.96 \\
\hline Met5 & P47169 & & & -0.34 & 0.27 & -0.06 & 0.04 & -0.28 & S 903 & 1 & 1.00 \\
\hline Gpd2 & P41911 & & & -0.40 & 0.20 & -0.12 & 0.13 & -0.28 & S 75 & 1 & 1.00 \\
\hline Ser33 & P40510 & & & -0.39 & 0.12 & -0.10 & 0.12 & -0.29 & T 31 & 2 & 0.97 \\
\hline Get2 & P40056 & & & 0.01 & 0.16 & 0.31 & 0.14 & -0.30 & T 55 & 1 & 0.64 \\
\hline Ser33 & \begin{tabular}{|l|} 
P40510 \\
\end{tabular} & & & -0.39 & 0.26 & -0.10 & 0.12 & -0.30 & S 22 & 2 & 0.99 \\
\hline Rpn13 & 013563 & & & -0.02 & 0.13 & 0.28 & 0.11 & -0.30 & S 135 & 1 & 1.00 \\
\hline Gpm1 & P00950 & & & -0.63 & 0.29 & -0.33 & 0.04 & $\mid-0.31$ & S 127 & 1 & 0.76 \\
\hline Vps13 & Q07878 & & & -0.21 & 0.13 & 0.10 & 0.10 & -0.31 & S 1362 & 1 & 0.90 \\
\hline Qri1 & P43123 & & & 0.04 & 0.17 & 0.36 & 0.11 & -0.32 & $S 218$ & 1 & 1.00 \\
\hline $\begin{array}{l}\text { Tdh1; } \\
\text { Tdh2; } \\
\text { Tdh3 }\end{array}$ & $\begin{array}{l}\text { P00360; } \\
\text { P00358; } \\
\text { P00359 }\end{array}$ & & & -0.26 & 0.09 & 0.06 & 0.29 & -0.32 & T 209 & 1 & 0.82 \\
\hline Chd1 & \begin{tabular}{|l} 
P32657 \\
\end{tabular} & & & -0.43 & 0.28 & -0.10 & 0.18 & -0.32 & S 1336 & 1 & 0.85 \\
\hline Spa2 & P23201 & & & -0.32 & 0.17 & 0.01 & 0.12 & $\mid-0.33$ & S 254 & 1 & 1.00 \\
\hline Fen1 & P25358 & & & -0.34 & 0.17 & -0.01 & 0.08 & $\mid-0.33$ & S 338 & 1 & 1.00 \\
\hline Gea2 & P39993 & & & -0.74 & 0.17 & -0.41 & 0.14 & -0.33 & $S 315$ & 1 & 0.97 \\
\hline $\begin{array}{l}\text { Hxt1; } \\
\text { Hxt3; } \\
\text { Hxt9; } \\
\text { Hxt11 }\end{array}$ & $\begin{array}{l}\text { P32465; } \\
\text { P32466; } \\
\text { P40885; } \\
\text { P54862 }\end{array}$ & & & $-0.81 \mid$ & 0.17 & -0.46 & 0.10 & -0.35 & S 3 & 1 & 0.99 \\
\hline Thr4 & \begin{tabular}{|l} 
P16120 \\
\end{tabular} & & & -0.14 & 0.27 & 0.21 & 0.02 & -0.35 & $S 411$ & 2 & 0.81 \\
\hline Thr4 & P16120 & & & -0.14 & 0.27 & 0.21 & 0.02 & $\mid-0.35$ & S 410 & 2 & 0.81 \\
\hline Bcy1 & \begin{tabular}{|l|} 
P07278 \\
\end{tabular} & & & -0.31 & 0.30 & 0.04 & 0.10 & -0.35 & S 145 & 1 & 0.92 \\
\hline
\end{tabular}


Supplementary Table 9. Continued 3.

\begin{tabular}{|c|c|c|c|c|c|c|c|c|c|c|c|}
\hline proteins & $\begin{array}{l}\text { protein } \\
\text { IDs }\end{array}$ & 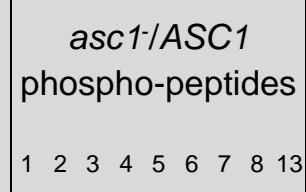 & \begin{tabular}{|ccccccccc}
\multicolumn{2}{c}{$\begin{array}{c}\text { asc } 1 / A S C 1 \\
\text { proteins }\end{array}$} \\
12 & 3 & 4 & 5 & 6 & 7 & 8 & 13
\end{tabular} \mid & $\begin{array}{l}\text { pho } \\
\text { pepti } \\
\varnothing\end{array}$ & ides & prot & SD & $\begin{array}{l}\text { diff. } \\
\text { phos- } \\
\text { prot }\end{array}$ & position & 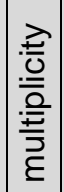 & 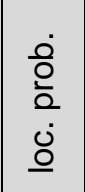 \\
\hline Fen1 & P25358 & & & -0.37 & 0.20 & -0.01 & 0.08 & $\mid-0.36$ & T 334 & 1 & 1.00 \\
\hline Sfk1 & P35735 & & & 0.00 & 0.25 & 0.36 & 0.13 & -0.36 & $T 124$ & 1 & 0.94 \\
\hline Prt1 & P06103 & & & -0.26 & 0.07 & 0.10 & 0.03 & $\mid-0.37$ & S 187 & 1 & 0.68 \\
\hline $\begin{array}{l}\text { Eno1; } \\
\text { Eno2 }\end{array}$ & $\begin{array}{l}\text { P00924; } \\
\text { P00925 }\end{array}$ & & & -0.09 & 0.16 & 0.28 & 0.11 & $\mid-0.37$ & S 10 & 1 & 1.00 \\
\hline Vtc2 & \begin{tabular}{|l|} 
P43585 \\
\end{tabular} & & & -0.08 & 0.06 & 0.30 & 0.19 & -0.38 & S 193 & 2 & 0.85 \\
\hline Met6 & P05694 & & & -0.52 & 0.16 & -0.14 & 0.06 & $\mid-0.38$ & $S 446$ & 1 & 0.99 \\
\hline Aro4 & \begin{tabular}{|l|} 
P32449 \\
\end{tabular} & & & -0.24 & 0.23 & 0.15 & 0.11 & -0.39 & S 4 & 1 & 0.98 \\
\hline Ett1 & Q08421 & & & -0.38 & 0.26 & 0.02 & 0.18 & -0.40 & S 41 & 1 & 0.98 \\
\hline Ypr091c & Q06833 & & & -0.89 & 0.14 & -0.49 & 0.24 & -0.40 & S 669 & 1 & 1.00 \\
\hline Ser33 & P40510 & & & -0.50 & 0.24 & -0.10 & 0.12 & -0.40 & S 2 & 1 & 1.00 \\
\hline Psk1 & P31374 & & & -0.48 & 0.23 & -0.07 & 0.14 & $\mid-0.41$ & S 1020 & 1 & 0.99 \\
\hline Vas1 & P07806 & & & -0.38 & 0.40 & 0.03 & 0.02 & -0.41 & S 294 & 1 & 1.00 \\
\hline Spc110 & P32380 & & & -0.36 & 0.14 & 0.05 & 0.20 & -0.41 & S 80 & 1 & 1.00 \\
\hline Gpd2 & P41911 & & & -0.55 & 0.01 & -0.12 & 0.13 & -0.43 & S 72 & 2 & 1.00 \\
\hline Hom3 & P10869 & & & -0.27 & 0.34 & 0.16 & 0.07 & $\mid-0.43$ & T 333 & 1 & 0.99 \\
\hline Aro8 & P53090 & & & 0.24 & 0.54 & 0.67 & 0.04 & -0.43 & $S 14$ & 1 & 0.53 \\
\hline Sui3 & P09064 & & & -0.27 & 0.13 & 0.17 & 0.12 & -0.44 & T 116 & 2 & 1.00 \\
\hline Sec31 & P38968 & & & -0.40 & 0.27 & 0.04 & 0.07 & -0.44 & S 999 & 1 & 1.00 \\
\hline Reb1 & P21538 & & & -0.49 & 0.26 & -0.04 & 0.26 & -0.45 & S 355 & 1 & 0.81 \\
\hline $\mathrm{Ncl} 1$ & P38205 & & & -0.69 & 0.70 & -0.22 & 0.06 & $\mid-0.47$ & S 424 & 1 & 0.98 \\
\hline Pkh3 & Q03306 & & & -0.41 & 0.09 & 0.06 & 0.05 & -0.47 & S 684 & 1 & 0.81 \\
\hline Vtc2 & P43585 & & & -0.19 & 0.07 & 0.30 & 0.19 & -0.49 & S 196 & 2 & 1.00 \\
\hline Pbp1 & P53297 & & & -0.49 & 0.23 & 0.00 & 0.13 & -0.49 & S 106 & 1 & 0.99 \\
\hline $\begin{array}{l}\text { Rpl7a; } \\
\text { Rpl7b }\end{array}$ & $\begin{array}{l}\text { P05737; } \\
\text { Q12213 }\end{array}$ & & & -0.56 & 0.25 & -0.07 & 0.03 & -0.49 & T 8;8 & 1 & 1.00 \\
\hline Cys3 & P31373 & & & 0.11 & 0.32 & 0.61 & 0.06 & -0.50 & S 40 & 1 & 0.97 \\
\hline Ty1 $b^{1}$ & P0C2I7 ${ }^{1}$ & & & 0.61 & 0.24 & 1.11 & 0.21 & -0.50 & $T 583^{1}$ & 1 & 0.56 \\
\hline Mlp1 & Q02455 & & & -0.69 & 0.22 & -0.18 & 0.18 & -0.51 & T 337 & 1 & 1.00 \\
\hline Vip1 & \begin{tabular}{|l|} 
Q06685 \\
\end{tabular} & & & -0.28 & 0.18 & 0.23 & 0.21 & -0.51 & S 31 & 1 & 0.98 \\
\hline Hom6 & P31116 & & & -0.35 & 0.21 & 0.17 & 0.02 & -0.52 & $S 240$ & 1 & 0.74 \\
\hline Sui3 & P09064 & & & -0.35 & 0.47 & 0.17 & 0.12 & -0.52 & S 112 & 2 & 0.96 \\
\hline Gpd2 & P41911 & & & -0.65 & 0.25 & -0.12 & 0.13 & -0.53 & S 75 & 2 & 1.00 \\
\hline Esc1 & Q03661 & & & -0.66 & 0.33 & -0.13 & 0.12 & $\mid-0.53$ & S 532 & 1 & 1.00 \\
\hline $\begin{array}{l}\text { Rps1a; } \\
\text { Rps1b }\end{array}$ & $\begin{array}{l}\text { P33442; } \\
\text { P23248 }\end{array}$ & & & -0.49 & 0.26 & 0.04 & 0.06 & -0.53 & S 236 & 1 & 1.00 \\
\hline $\begin{array}{l}\text { Rpl7a; } \\
\text { Rpl7b }\end{array}$ & $\begin{array}{l}\text { P05737; } \\
\text { Q12213 }\end{array}$ & & & -0.61 & 0.05 & -0.07 & 0.03 & $\mid-0.54$ & T 8;8 & 2 & 1.00 \\
\hline $\begin{array}{l}\text { Rpl7a; } \\
\text { Rpl7b }\end{array}$ & \begin{tabular}{|l} 
P05737; \\
Q12213
\end{tabular} & & & -0.61 & 0.05 & -0.07 & 0.03 & $\mid-0.54$ & S 11 & 2 & 1.00 \\
\hline Pdr1 & P12383 & & & -0.71 & 0.33 & -0.17 & 0.08 & -0.54 & S 942 & 1 & 0.81 \\
\hline Cdc3 & P32457 & & & -0.31 & 0.40 & 0.24 & 0.08 & $\mid-0.54$ & S 77 & 1 & 0.93 \\
\hline $\mathrm{Ncl} 1$ & P38205 & & & -0.77 & 0.21 & -0.22 & 0.06 & -0.55 & S 423 & 1 & 0.90 \\
\hline Cho1 & P08456 & & & -0.41 & 0.14 & 0.14 & 0.18 & -0.55 & S 50 & 2 & 0.95 \\
\hline Cho1 & P08456 & & & -0.41 & 0.21 & 0.14 & 0.18 & -0.55 & S 47 & 2 & 1.00 \\
\hline Cho1 & P08456 & & & -0.41 & 0.22 & 0.14 & 0.18 & -0.55 & S 46 & 2 & 1.00 \\
\hline Pbp1 & \begin{tabular}{|l|} 
P53297 \\
\end{tabular} & & & -0.56 & 0.12 & 0.00 & 0.13 & -0.55 & $S 104$ & 1 & 0.99 \\
\hline
\end{tabular}


Supplementary Table 9. Continued 4.

\begin{tabular}{|c|c|c|c|c|c|c|c|c|c|c|c|}
\hline proteins & $\begin{array}{l}\text { protein } \\
\text { IDs }\end{array}$ & 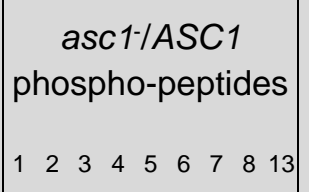 & \begin{tabular}{cccccccc}
\multicolumn{4}{c}{$\begin{array}{c}\text { asc1-/ASC1 } \\
\text { proteins }\end{array}$} \\
123 & 4 & 5 & 6 & 7 & 8 & 13
\end{tabular} \mid & $\begin{array}{l}\text { pho } \\
\text { pepti } \\
\varnothing\end{array}$ & ides- & prot & SD & $\begin{array}{l}\text { diff. } \\
\text { phos- } \\
\text { prot }\end{array}$ & position & 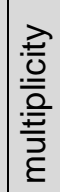 & $\begin{array}{l}\text { 음 } \\
\text { 음 } \\
\text { 음 }\end{array}$ \\
\hline Pfy1 & P07274 & & & -0.56 & 0.22 & 0.00 & 0.09 & -0.56 & S 2 & 1 & 1.00 \\
\hline Ent2 & Q05785 & & & -0.31 & 0.13 & 0.25 & 0.28 & -0.56 & S 173 & 1 & 0.88 \\
\hline Zeo1 & Q08245 & & & -0.73 & 0.36 & -0.16 & 0.09 & -0.56 & T 49 & 1 & 1.00 \\
\hline Apa1 & P16550 & & & -0.53 & 0.19 & 0.03 & 0.07 & -0.56 & T 60 & 1 & 1.00 \\
\hline Gpm1 & P00950 & & & -0.90 & 0.34 & -0.33 & 0.04 & -0.57 & S 128 & 1 & 0.80 \\
\hline Acc1 & Q00955 & & & -1.14 & 0.27 & -0.55 & 0.09 & -0.59 & S 1157 & 1 & 1.00 \\
\hline Hom3 & P10869 & & & -0.44 & 0.21 & 0.16 & 0.07 & -0.59 & S 332 & 1 & 0.94 \\
\hline Tsa1 & P34760 & & & -1.23 & 0.03 & -0.64 & 0.04 & -0.60 & T 174 & 1 & 1.00 \\
\hline Sod1 & P00445 & & & -0.81 & 0.15 & -0.19 & 0.09 & -0.62 & S 39 & 1 & 1.00 \\
\hline Ura2 & P07259 & & & -1.20 & 0.28 & -0.57 & 0.06 & -0.62 & T 1859 & 1 & 0.77 \\
\hline Pup2 & P32379 & & & -0.34 & 0.20 & 0.31 & 0.04 & -0.65 & S 56 & 1 & 0.89 \\
\hline Prr1 & P28708 & & & -0.51 & 0.22 & 0.14 & 0.18 & -0.65 & S 132 & 1 & 1.00 \\
\hline $\begin{array}{l}\text { Rps19a; } \\
\text { Rps19b }\end{array}$ & $\begin{array}{l}\text { P07280; } \\
\text { P07281 }\end{array}$ & & & -0.71 & 0.36 & -0.06 & 0.05 & -0.65 & S 117 & 1 & 1.00 \\
\hline Ssd1 & \begin{tabular}{|l|} 
P24276 \\
\end{tabular} & & & -0.71 & 0.12 & -0.06 & 0.14 & -0.65 & S 231 & 1 & 0.96 \\
\hline Trm2 & P33753 & & & -0.62 & 0.17 & 0.04 & 0.13 & -0.66 & 598 & 1 & 0.95 \\
\hline Rad16 & P31244 & & & -0.67 & 0.22 & -0.01 & 0.23 & -0.66 & S 25 & 1 & 1.00 \\
\hline Sec21 & \begin{tabular}{|l|} 
P32074 \\
\end{tabular} & & & -0.82 & 0.27 & -0.15 & 0.07 & -0.67 & T 638 & 1 & 1.00 \\
\hline Trm2 & P33753 & & & -0.63 & 0.11 & 0.04 & 0.13 & -0.68 & S 92 & 1 & 0.95 \\
\hline Rsc2 & Q06488 & & & -1.02 & 0.25 & -0.34 & 0.22 & -0.68 & S 682 & 1 & 1.00 \\
\hline Abp1 & P15891 & & & 0.08 & 0.52 & 0.78 & 0.16 & -0.70 & S 167 & 1 & 0.94 \\
\hline Ent3 & P47160 & & & -0.43 & 0.34 & 0.27 & 0.13 & -0.70 & $S 2$ & 1 & 1.00 \\
\hline Rad16 & P31244 & & & -0.74 & 0.47 & -0.01 & 0.23 & -0.73 & $S 78$ & 1 & 0.99 \\
\hline Vps1 & P21576 & & & -0.48 & 0.20 & 0.26 & 0.07 & -0.73 & S 599 & 1 & 1.00 \\
\hline Rps7b & P48164 & & & -0.47 & 0.41 & 0.27 & 0.05 & -0.74 & S 31 & 1 & 0.87 \\
\hline Trm2 & P33753 & & & -0.69 & 0.02 & 0.04 & 0.13 & -0.74 & T 96 & 1 & 0.87 \\
\hline Trm2 & P33753 & & & -0.70 & 0.29 & 0.04 & 0.13 & -0.74 & S 93 & 1 & 0.97 \\
\hline Gpd2 & P41911 & & & -0.89 & 0.19 & -0.12 & 0.13 & -0.77 & S 70 & 2 & 0.97 \\
\hline Orm2 & Q06144 & & & -0.37 & 0.18 & 0.41 & 0.14 & -0.77 & S 9 & 1 & 1.00 \\
\hline Pup2 & P32379 & & & -0.47 & 0.19 & 0.31 & 0.04 & -0.78 & T 55 & 1 & 0.96 \\
\hline Cdc60 & P26637 & & & -0.45 & 0.34 & 0.33 & 0.05 & -0.78 & T 142 & 1 & 1.00 \\
\hline Rps7b & P48164 & & & -0.52 & 0.20 & 0.27 & 0.05 & -0.78 & S 30 & 1 & 0.90 \\
\hline Rad9 & P14737 & & & -0.37 & 0.00 & 0.42 & 0.07 & -0.79 & S 494 & 1 & 0.81 \\
\hline Sui3 & P09064 & & & -0.63 & 0.30 & 0.17 & 0.12 & -0.80 & T 116 & 1 & 1.00 \\
\hline Sum1 & P46676 & & & -0.68 & 0.00 & 0.12 & 0.17 & -0.80 & S 736 & 1 & 0.71 \\
\hline Sui3 & P09064 & & & -0.63 & 0.10 & 0.17 & 0.12 & -0.80 & S 118 & 1 & 0.69 \\
\hline Yhr020w & P38708 & & & -0.73 & 0.16 & 0.09 & 0.06 & -0.82 & $T 38$ & 1 & 0.50 \\
\hline Yhr020w & P38708 & & & -0.73 & 0.16 & 0.09 & 0.06 & -0.82 & S 36 & 1 & 0.50 \\
\hline Zeo1 & Q08245 & & & -0.99 & 0.41 & -0.16 & 0.09 & -0.83 & T 49 & 2 & 1.00 \\
\hline Zeo1 & Q08245 & & & -0.99 & 0.41 & -0.16 & 0.09 & -0.83 & S 40 & 2 & 1.00 \\
\hline Gcs1 & P35197 & & & -0.55 & 0.32 & 0.29 & 0.09 & -0.85 & S 157 & 1 & 0.81 \\
\hline Ura2 & P07259 & & & -1.43 & 0.18 & -0.57 & 0.06 & -0.86 & S 1857 & 1 & 0.98 \\
\hline Ty $1 b^{2}$ & P0C2I7 ${ }^{2}$ & & & 0.25 & 0.22 & 1.11 & 0.21 & -0.86 & $S 445^{2}$ & 1 & 0.67 \\
\hline Def1 & P35732 & & & -0.96 & 0.49 & 0.00 & 0.19 & -0.95 & T 258 & 1 & 0.90 \\
\hline $\begin{array}{l}\text { Tdh1; } \\
\text { Tdh2; } \\
\text { Tdh3 }\end{array}$ & $\begin{array}{l}\text { P00360; } \\
\text { P00358; } \\
\text { P00359 }\end{array}$ & & & -0.94 & 0.21 & 0.06 & 0.29 & -1.01 & S 201 & 1 & 1.00 \\
\hline Gvp36 & P40531 & & & -1.06 & 0.48 & -0.04 & 0.07 & -1.03 & $S 2$ & 1 & 1.00 \\
\hline
\end{tabular}


Supplementary Table 9. Continued 5.

\begin{tabular}{|c|c|c|c|c|c|c|c|c|c|c|c|}
\hline proteins & $\begin{array}{l}\text { protein } \\
\text { IDs }\end{array}$ & 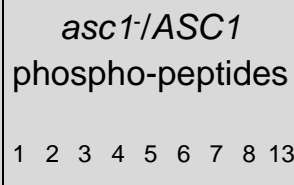 & 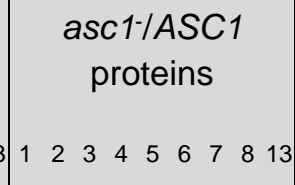 & $\begin{array}{l}\text { phi } \\
\text { pept } \\
\varnothing\end{array}$ & SD & prot & SD & $\begin{array}{l}\text { diff. } \\
\text { phos- } \\
\text { prot }\end{array}$ & position & 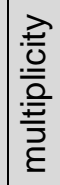 & $\begin{array}{l}\text { 음 } \\
\text { 음 } \\
\text { 음 }\end{array}$ \\
\hline Abp1 & P15891 & & & -0.25 & 0.26 & 0.78 & 0.16 & -1.03 & S 169 & 1 & 1.00 \\
\hline Hsp42 & Q12329 & & & -0.37 & 0.19 & 0.69 & 0.35 & -1.06 & S 223 & 1 & 0.99 \\
\hline Def1 & P35732 & & & -1.08 & 0.10 & 0.00 & 0.19 & -1.08 & S 260 & 1 & 1.00 \\
\hline Tif4632 & P39936 & & & -0.97 & 0.27 & 0.21 & 0.12 & -1.18 & T 196 & 1 & 1.00 \\
\hline Bre5 & P53741 & & & -1.34 & 0.45 & -0.14 & 0.24 & -1.20 & S 282 & 1 & 1.00 \\
\hline Grx2 & P17695 & & & -1.05 & 0.45 & 0.25 & 0.31 & -1.30 & S 94 & 1 & 0.94 \\
\hline Ctr9 & P89105 & & & -1.15 & 0.11 & 0.16 & 0.07 & -1.31 & S 1017 & 1 & 1.00 \\
\hline Puf3 & Q07807 & & & -1.03 & 0.20 & 0.31 & 0.11 & -1.34 & S 86 & 1 & 1.00 \\
\hline Ugp1 & P32861 & & & -1.26 & 0.63 & 0.16 & 0.15 & -1.42 & Y 13 & 1 & 0.55 \\
\hline Grx2 & P17695 & & & -1.17 & 0.43 & 0.25 & 0.31 & -1.42 & S 91 & 1 & 1.00 \\
\hline Cdc28 & P00546 & & & -0.76 & 0.59 & 0.70 & 0.14 & -1.46 & Y 19 & 1 & 0.98 \\
\hline Abp1 & P15891 & & & -0.76 & 0.54 & 0.78 & 0.16 & 1.53 & T 181 & 2 & 1.00 \\
\hline Abp1 & P15891 & & & -0.76 & 0.54 & 0.78 & 0.16 & 1.53 & S 183 & 2 & 1.00 \\
\hline Egd1 & Q02642 & & & -3.48 & 0.07 & -0.12 & 0.12 & -3.36 & T 151 & 1 & 1.00 \\
\hline \multicolumn{12}{|c|}{ phospho-peptides with no proteome value } \\
\hline Edc1 & P53080 & & & 2.15 & 0.11 & $\mathrm{NaN}$ & $\mathrm{NaN}$ & $\mathrm{NaN}$ & S 82 & 1 & 0.99 \\
\hline Ren1 & P36054 & & & 0.91 & 0.21 & $\mathrm{NaN}$ & $\mathrm{NaN}$ & $\mathrm{NaN}$ & S 117 & 2 & 1.00 \\
\hline Ren1 & P36054 & & & 0.91 & 0.21 & $\mathrm{NaN}$ & $\mathrm{NaN}$ & $\mathrm{NaN}$ & S 113 & 2 & 1.00 \\
\hline Nte1 & Q04958 & & & 0.83 & 0.10 & $\mathrm{NaN}$ & $\mathrm{NaN}$ & $\mathrm{NaN}$ & S 634 & 1 & 0.99 \\
\hline $\mathrm{Ty}_{2 \mathrm{a}^{3}}$ & Q99303 $^{3}$ & & & 0.81 & 0.48 & $\mathrm{NaN}$ & $\mathrm{NaN}$ & $\mathrm{NaN}$ & $S 424$ & 1 & 0.99 \\
\hline Aim3 & P38266 & & & 0.79 & 0.21 & $\mathrm{NaN}$ & $\mathrm{NaN}$ & $\mathrm{NaN}$ & S 843 & 1 & 0.93 \\
\hline Nnk1 & P36003 & & & 0.70 & 0.30 & $\mathrm{NaN}$ & $\mathrm{NaN}$ & $\mathrm{NaN}$ & Y 739 & 1 & 1.00 \\
\hline Pgm3 & Q03262 & & & 0.69 & 0.25 & $\mathrm{NaN}$ & $\mathrm{NaN}$ & $\mathrm{NaN}$ & $T 156$ & 1 & 0.66 \\
\hline Dig2 & Q03373 & & & 0.64 & 0.27 & $\mathrm{NaN}$ & $\mathrm{NaN}$ & $\mathrm{NaN}$ & T 83 & 1 & 0.76 \\
\hline Pgm3 & \begin{tabular}{|l|} 
Q03262 \\
\end{tabular} & & & 0.64 & 0.27 & $\mathrm{NaN}$ & $\mathrm{NaN}$ & $\mathrm{NaN}$ & S 158 & 1 & 0.97 \\
\hline Rrp36 & Q12481 & & & 0.63 & 0.34 & $\mathrm{NaN}$ & $\mathrm{NaN}$ & $\mathrm{NaN}$ & S 14 & 1 & 1.00 \\
\hline Dig2 & Q03373 & & & 0.62 & 0.19 & $\mathrm{NaN}$ & $\mathrm{NaN}$ & $\mathrm{NaN}$ & T 82 & 1 & 0.94 \\
\hline Stb1 & P42845 & & & 0.60 & 0.36 & $\mathrm{NaN}$ & $\mathrm{NaN}$ & $\mathrm{NaN}$ & T 99 & 1 & 1.00 \\
\hline Stb1 & P42845 & & & 0.58 & 0.36 & $\mathrm{NaN}$ & $\mathrm{NaN}$ & $\mathrm{NaN}$ & S 72 & 1 & 1.00 \\
\hline Ppq1 & P32945 & & & 0.58 & 0.20 & $\mathrm{NaN}$ & $\mathrm{NaN}$ & $\mathrm{NaN}$ & S 208 & 1 & 0.89 \\
\hline Ysc84 & P32793 & & & 0.58 & 0.01 & $\mathrm{NaN}$ & $\mathrm{NaN}$ & $\mathrm{NaN}$ & S 301 & 1 & 1.00 \\
\hline Gde1 & Q02979 & & & 0.56 & 0.09 & $\mathrm{NaN}$ & $\mathrm{NaN}$ & $\mathrm{NaN}$ & S 254 & 1 & 0.94 \\
\hline Air2 & Q12476 & & & 0.52 & 0.17 & $\mathrm{NaN}$ & $\mathrm{NaN}$ & $\mathrm{NaN}$ & S 49 & 1 & 1.00 \\
\hline Den1 & Q12395 & & & 0.52 & 0.08 & $\mathrm{NaN}$ & $\mathrm{NaN}$ & $\mathrm{NaN}$ & S 12 & 1 & 1.00 \\
\hline Ren1 & \begin{tabular}{|l|} 
P36054 \\
\end{tabular} & & & 0.47 & 0.13 & $\mathrm{NaN}$ & $\mathrm{NaN}$ & $\mathrm{NaN}$ & S 117 & 1 & 1.00 \\
\hline She3 & P38272 & & & 0.46 & 0.19 & $\mathrm{NaN}$ & $\mathrm{NaN}$ & $\mathrm{NaN}$ & S 361 & 1 & 1.00 \\
\hline Air2 & Q12476 & & & 0.43 & 0.13 & $\mathrm{NaN}$ & $\mathrm{NaN}$ & $\mathrm{NaN}$ & T 16 & 1 & 0.99 \\
\hline Bud21 & Q08492 & & & 0.43 & 0.14 & $\mathrm{NaN}$ & $\mathrm{NaN}$ & $\mathrm{NaN}$ & S 144 & 1 & 1.00 \\
\hline Pol1 & P13382 & & & 0.42 & 0.23 & $\mathrm{NaN}$ & $\mathrm{NaN}$ & $\mathrm{NaN}$ & S 170 & 1 & 0.95 \\
\hline Kha1 & P40309 & & & 0.40 & 0.12 & $\mathrm{NaN}$ & $\mathrm{NaN}$ & $\mathrm{NaN}$ & S 557 & 1 & 1.00 \\
\hline Pik1 & P39104 & & & 0.38 & 0.15 & $\mathrm{NaN}$ & $\mathrm{NaN}$ & $\mathrm{NaN}$ & S 236 & 1 & 0.96 \\
\hline Fip1 & P45976 & & & 0.37 & 0.22 & $\mathrm{NaN}$ & $\mathrm{NaN}$ & $\mathrm{NaN}$ & S 15 & 1 & 1.00 \\
\hline Ent1 & Q12518 & & & 0.36 & 0.26 & $\mathrm{NaN}$ & $\mathrm{NaN}$ & $\mathrm{NaN}$ & S 327 & 1 & 0.96 \\
\hline She3 & \begin{tabular}{|l|} 
P38272 \\
\end{tabular} & & & 0.35 & 0.10 & $\mathrm{NaN}$ & $\mathrm{NaN}$ & $\mathrm{NaN}$ & S 394 & 1 & 0.73 \\
\hline Rho4 & Q00246 & & & 0.30 & 0.29 & $\mathrm{NaN}$ & $\mathrm{NaN}$ & $\mathrm{NaN}$ & S 58 & 1 & 1.00 \\
\hline Sng1 & P46950 & & & 0.30 & 0.12 & $\mathrm{NaN}$ & $\mathrm{NaN}$ & $\mathrm{NaN}$ & T 91 & 1 & 1.00 \\
\hline Vid27 & P40157 & & & 0.29 & 0.16 & $\mathrm{NaN}$ & $\mathrm{NaN}$ & $\mathrm{NaN}$ & S 222 & 1 & 1.00 \\
\hline
\end{tabular}


Supplementary Table 9. Continued 6.

\begin{tabular}{|c|c|c|c|c|c|c|c|c|c|c|c|}
\hline proteins & $\begin{array}{l}\text { protein } \\
\text { IDs }\end{array}$ & 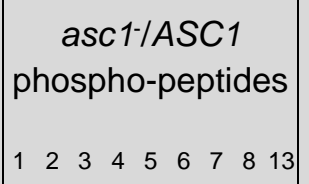 & 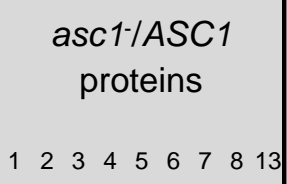 & $\begin{array}{l}\text { pho } \\
\text { pepti } \\
\varnothing\end{array}$ & & prote & SD & $\begin{array}{l}\text { diff. } \\
\text { phos- } \\
\text { prot }\end{array}$ & position & 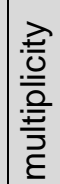 & $\begin{array}{l}\text { 을 } \\
\text { 흥 } \\
\text { 으 }\end{array}$ \\
\hline Ypi1 & P43587 & & & 0.28 & 0.10 & $\mathrm{NaN}$ & $\mathrm{NaN}$ & $\mathrm{NaN}$ & S 131 & 1 & 0.73 \\
\hline Bbp1 & Q12365 & & & -0.32 & 0.22 & $\mathrm{NaN}$ & $\mathrm{NaN}$ & $\mathrm{NaN}$ & T 7 & 1 & 1.00 \\
\hline Taf3 & Q12297 & & & -0.32 & 0.15 & $\mathrm{NaN}$ & $\mathrm{NaN}$ & $\mathrm{NaN}$ & S 346 & 1 & 0.76 \\
\hline Ecm21 & P38167 & & & -0.47 & 0.13 & $\mathrm{NaN}$ & $\mathrm{NaN}$ & $\mathrm{NaN}$ & S 140 & 1 & 1.00 \\
\hline Ume6 & P39001 & & & -0.50 & 0.19 & $\mathrm{NaN}$ & $\mathrm{NaN}$ & $\mathrm{NaN}$ & S 645 & 1 & 1.00 \\
\hline Mdv1 & P47025 & & & -0.50 & 0.23 & $\mathrm{NaN}$ & $\mathrm{NaN}$ & $\mathrm{NaN}$ & S 376 & 1 & 1.00 \\
\hline Yel043w & P32618 & & & -0.55 & 0.27 & $\mathrm{NaN}$ & $\mathrm{NaN}$ & $\mathrm{NaN}$ & S 802 & 1 & 1.00 \\
\hline Yap1 & P19880 & & & -0.60 & 0.32 & $\mathrm{NaN}$ & $\mathrm{NaN}$ & $\mathrm{NaN}$ & S 14 & 1 & 1.00 \\
\hline Bni1 & P41832 & & & -0.62 & 0.22 & $\mathrm{NaN}$ & $\mathrm{NaN}$ & $\mathrm{NaN}$ & S 1889 & 1 & 0.99 \\
\hline Sch9 & P11792 & & & -0.64 & 0.12 & $\mathrm{NaN}$ & $\mathrm{NaN}$ & $\mathrm{NaN}$ & S 726 & 1 & 1.00 \\
\hline Ycr023c & P25351 & & & -0.72 & 0.16 & $\mathrm{NaN}$ & $\mathrm{NaN}$ & $\mathrm{NaN}$ & S 313 & 1 & 1.00 \\
\hline Par32 & Q12515 & & & -0.75 & 0.49 & $\mathrm{NaN}$ & $\mathrm{NaN}$ & $\mathrm{NaN}$ & S 39 & 1 & 1.00 \\
\hline Gat1 & P43574 & & & -0.82 & 0.27 & $\mathrm{NaN}$ & $\mathrm{NaN}$ & $\mathrm{NaN}$ & S 262 & 1 & 0.98 \\
\hline Vid27 & P40157 & & & -0.94 & 0.30 & $\mathrm{NaN}$ & $\mathrm{NaN}$ & $\mathrm{NaN}$ & T 220 & 2 & 1.00 \\
\hline Vid27 & P40157 & & & -0.94 & 0.30 & $\mathrm{NaN}$ & $\mathrm{NaN}$ & $\mathrm{NaN}$ & S 222 & 2 & 1.00 \\
\hline Meh1 & Q02205 & & & -0.95 & 0.31 & $\mathrm{NaN}$ & $\mathrm{NaN}$ & $\mathrm{NaN}$ & S 146 & 1 & 0.99 \\
\hline Vtc3 & Q02725 & & & -0.99 & 0.28 & $\mathrm{NaN}$ & $\mathrm{NaN}$ & $\mathrm{NaN}$ & S 198 & 1 & 1.00 \\
\hline Rho5 & \begin{tabular}{|l|} 
P53879 \\
\end{tabular} & & & -1.03 & 0.26 & $\mathrm{NaN}$ & $\mathrm{NaN}$ & $\mathrm{NaN}$ & S 223 & 1 & 1.00 \\
\hline Mnr2 & P35724 & & & -1.05 & 0.17 & $\mathrm{NaN}$ & $\mathrm{NaN}$ & $\mathrm{NaN}$ & T 177 & 1 & 0.99 \\
\hline Nrg2 & P38082 & & & -1.08 & 0.61 & $\mathrm{NaN}$ & $\mathrm{NaN}$ & $\mathrm{NaN}$ & S 100 & 1 & 0.77 \\
\hline Kns1 & P32350 & & & -1.10 & 0.51 & $\mathrm{NaN}$ & $\mathrm{NaN}$ & $\mathrm{NaN}$ & T 562 & 1 & 1.00 \\
\hline Mnr2 & P35724 & & & -1.14 & 0.21 & $\mathrm{NaN}$ & $\mathrm{NaN}$ & $\mathrm{NaN}$ & S 152 & 1 & 1.00 \\
\hline Nrg2 & P38082 & & & -1.6 & 0.41 & $\mathrm{NaN}$ & $\mathrm{NaN}$ & $\mathrm{NaN}$ & $T 99$ & 1 & 0.95 \\
\hline Mep2 & P41948 & & & -1.67 & 0.13 & $\mathrm{NaN}$ & $\mathrm{NaN}$ & $\mathrm{NaN}$ & $S 460$ & 1 & 0.73 \\
\hline Mep2 & P41948 & & & -1.76 & 0.21 & $\mathrm{NaN}$ & $\mathrm{NaN}$ & $\mathrm{NaN}$ & T 459 & 1 & 0.63 \\
\hline Tpo1 & Q07824 & & & -1.71 & 0.51 & $\mathrm{NaN}$ & $\mathrm{NaN}$ & $\mathrm{NaN}$ & S 72 & 1 & 1.00 \\
\hline \multicolumn{12}{|c|}{ phospho-peptides with one or two proteome value(s) } \\
\hline Dbf2 & P22204 & & & 0.51 & 0.12 & 0.21 & & 0.30 & S 374 & 1 & 1.00 \\
\hline Kin82 & P25341 & & & 0.37 & 0.10 & 0.09 & & 0.28 & T 499 & 1 & 0.76 \\
\hline Dbf20 & P32328 & & & -0.12 & 0.06 & 0.22 & & -0.34 & S 366 & 1 & 1.00 \\
\hline Pct1 & P13259 & & & -0.23 & 0.22 & 0.15 & 0.62 & -0.38 & S 346 & 1 & 1.00 \\
\hline Fhl1 & P39521 & & & -0.52 & 0.19 & -0.12 & & -0.40 & S 264 & 1 & 1.00 \\
\hline Ssn2 & P38931 & & & -0.51 & 0.23 & -0.01 & & -0.50 & S 375 & 1 & 1.00 \\
\hline Fhl1 & P39521 & & & -0.65 & 0.15 & -0.12 & & -0.53 & S 44 & 1 & 0.97 \\
\hline Glc8 & P41818 & & & -0.21 & 0.10 & 0.35 & & -0.57 & S 12 & 1 & 1.00 \\
\hline Cue4 & Q04201 & & & -0.65 & 0.41 & -0.01 & & -0.64 & S 48 & 1 & 1.00 \\
\hline Yjl070c & P40361 & & & -0.48 & 0.05 & 0.17 & & $\mid-0.65$ & S 43 & 1 & 1.00 \\
\hline Yjl070c & P40361 & & & -0.50 & 0.09 & 0.17 & & -0.67 & S 41 & 1 & 0.99 \\
\hline Kin2 & P13186 & & & -0.49 & 0.14 & 0.38 & & -0.87 & S 549 & 1 & 1.00 \\
\hline
\end{tabular}

${ }^{1}$ Ty1b-Lr4;Ty1b-Pr1;Ty1b-Gr2;Ty1b-Pr2;Ty1b-Er1;Ty1b-MI2;Ty1b-OI;Ty1b-Jr1;Ty1b-A;Ty1b-Ml1;Ty1bPI;Ty1b-Lr2

P0C2I7;P0C2J0;Q03612;Q03434;Q12273;P47098;013527; 583; 1142; 1143

${ }^{2}$ Ty1b-Lr4;Ty1b-Pr1;Ty1b-Gr2;Ty1b-Pr2;Ty1b-Er1;Ty1b-MI2;Ty1b-OI;Ty1b-Jr1;Ty1b-A;Ty1b-Ml1;Ty1b-

PI;Ty1b-Lr2

P0C2I7;P0C2J0;Q03612;Q03434;Q12273;P47098;O13527; 445; 1004; 1005

${ }^{3}$ Ty2a-Dr3;Ty2a-C;Ty2a-Or1;Ty2a-Lr2;Ty2a-Gr2;Ty2a-F

Q99303;P25383;Q12439;P0C2J6;P0CX62;P0CX61 
Supplementary Table 10. SILAC-based phospho-proteome data for the comparison of the

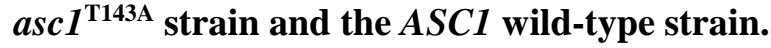

The table lists the proteins that fulfilled the criteria described in Supplementary Table 3. The replicates are numbered 1-3 and 4, 8, and 12 according to Figure 21. $\left(\mathrm{A}=\operatorname{ascl} 1^{\mathrm{T} 143 \mathrm{~A}} / A S C l ;\right.$ Aux $=$ $A S C 1^{\text {Aux }} / A S C 1 ; \varnothing=$ mean of phospho-peptide (PP) and protein (Prot) $\log _{2}$ SILAC-ratios for A and Aux; A Ø PP - Prot = mean of PP ratios minus mean of Prot ratios for A; Aux $\varnothing$ PP - Prot = mean of

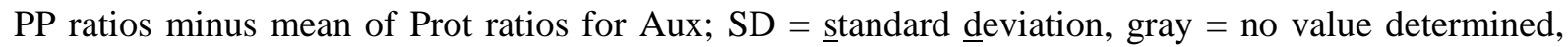
position $=$ phosphorylated amino acid residue, multiplicity $=$ number of phosphorylations within the identified phospho-peptide; loc. prob. = localization probability

\begin{tabular}{|c|c|c|c|c|c|c|c|c|c|c|c|c|c|c|c|}
\hline proteins & $\begin{array}{c}\text { protein } \\
\text { IDs }\end{array}$ & $\begin{array}{ccc} & \begin{array}{c}\text { P } \\
\text { PP }\end{array} \\
1 & 2 & 3\end{array}$ & 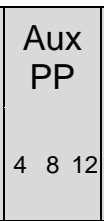 & $\begin{array}{c}\text { A } \\
\text { Prot } \\
1123\end{array}$ & 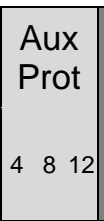 & $\begin{array}{c}\text { A } \\
\mathrm{PP} \\
\varnothing \\
\mathrm{SD}\end{array}$ & $\begin{array}{c}\text { Aux } \\
\text { PP } \\
\varnothing \\
\text { SD }\end{array}$ & $\begin{array}{c}\text { A } \\
\text { Prot } \\
\varnothing \\
\text { SD }\end{array}$ & $\begin{array}{c}\text { Aux } \\
\text { Prot } \\
\varnothing \\
\text { SD }\end{array}$ & \begin{tabular}{|c|}
$1:$ \\
A \\
$\varnothing$ \\
PP- \\
Prot
\end{tabular} & $\begin{array}{c}2: \\
\text { Aux } \\
\varnothing \\
\text { PP- } \\
\text { Prot }\end{array}$ & $1-2$ & 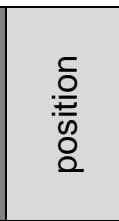 & 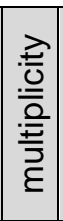 & 은 \\
\hline Nth1 & 23 & & & & & & & & & 0.29 & $-0.05 \mid$ & 0.34 & S 60 & 1 & 1.00 \\
\hline Ent3 & 7160 & & & & & & & $\begin{array}{l}-0.07 \\
\pm 0.06\end{array}$ & $\begin{array}{r}-0.03 \\
\pm 0.08\end{array}$ & -0.12 & 0.17 & -0.30 & S 2 & 1 & .0 \\
\hline Met17 & P06106 & & & & & & $\begin{array}{c}0.02 \\
\pm 0.01\end{array}$ & $\begin{array}{c}0.03 \\
\pm 0.05\end{array}$ & $\begin{array}{r}-0.02 \\
\pm 0.05\end{array}$ & $-0.27 \mid$ & 0.03 & -0.30 & 34 & 1 & 00 \\
\hline $\begin{array}{l}\text { Rps1a; } \\
\text { Rps1b }\end{array}$ & $\begin{array}{l}\text { P334L } \\
\text { P232 }\end{array}$ & & & & & $\begin{array}{l}-0.22 \\
\pm 0.06\end{array}$ & $\begin{array}{c}0.20 \\
\pm 0.10\end{array}$ & $\begin{array}{l}-0.05 \\
\pm 0.01\end{array}$ & $\begin{array}{l}-0.01 \\
\pm 0.06\end{array}$ & $|-0.17|$ & 0.21 & -0.38 & S 236 & 1 & 1.00 \\
\hline Saf1 & $\mathrm{P}$ & & & & & & $\begin{array}{c}0.44 \\
\pm 0.02\end{array}$ & $\begin{array}{l}-0.09 \\
\pm 0.01\end{array}$ & & 0.14 & 0.56 & -0.42 & S 16 & 1 & 1.00 \\
\hline Sec31 & 38968 & & & & & & & $\begin{array}{r}0.06 \\
\pm 0.02 \\
\end{array}$ & $\begin{array}{r}-0.02 \\
\pm 0.11 \\
\end{array}$ & -0.32 & 0.27 & -0.59 & S 980 & 1 & 1.0 \\
\hline
\end{tabular}

Supplementary Table 11. SILAC-based phospho-proteome data for the comparison of the

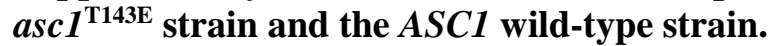

The table lists the proteins that fulfilled the criteria described in Supplementary Table 3. The replicates are numbered 5-7 and 4, 8, and 12 according to Figure 21. $\left(\mathrm{E}=a s c 1^{\mathrm{T} 143 \mathrm{E}} / A S C 1\right.$; Aux $=A S C 1^{\mathrm{Aux}} / A S C 1$; $\varnothing=$ mean of phospho-peptide (PP) and protein (Prot) $\log _{2}$ SILAC-ratios for E and Aux; E Ø PP - Prot $=$ mean of PP ratios minus mean of Prot ratios for E; Aux $\emptyset \mathrm{PP}-$ Prot $=$ mean of PP ratios minus mean of Prot ratios for Aux; SD = standard deviation, gray $=$ no value determined, position $=$ phosphorylated amino acid residue, multiplicity $=$ number of phosphorylations within the identified phospho-peptide; loc. prob. = localization probability

\begin{tabular}{|c|c|c|c|c|c|c|c|c|c|c|c|c|c|c|c|}
\hline proteins & $\begin{array}{l}\text { protein } \\
\text { IDs }\end{array}$ & $\begin{array}{c}E \\
P P \\
567\end{array}$ & $\begin{array}{l}\text { Aux } \\
\text { PP } \\
4812\end{array}$ & $\begin{array}{c}E \\
\text { Prot } \\
567\end{array}$ & $\begin{array}{l}\text { Aux } \\
\text { Prot } \\
48812\end{array}$ & $\begin{array}{c}\mathrm{E} \\
\mathrm{PP} \\
\varnothing \\
\mathrm{SD}\end{array}$ & $\begin{array}{c}\text { Aux } \\
\text { PP } \\
\varnothing \\
\text { SD }\end{array}$ & $\begin{array}{c}E \\
\text { Prot } \\
\varnothing \\
\text { SD }\end{array}$ & $\begin{array}{c}\text { Aux } \\
\text { Prot } \\
\varnothing \\
\text { SD }\end{array}$ & $\begin{array}{c}1: \\
\mathrm{E} \\
\varnothing \\
\text { PP- } \\
\text { Prot }\end{array}$ & $\begin{array}{c}2: \\
\text { Aux } \\
\varnothing \\
\text { PP- } \\
\text { Prot }\end{array}$ & $1-2$ & $\begin{array}{l}\text { 을 } \\
\frac{\bar{t}}{00} \\
\stackrel{2}{2}\end{array}$ & 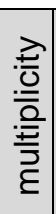 & $\begin{array}{l}\text { 응 } \\
\text { 응 } \\
\text { 으 }\end{array}$ \\
\hline Sbp1 & P10080 & & & & & $\begin{array}{c}1.40 \\
\pm 0.10\end{array}$ & $\begin{array}{c}0.29 \\
\pm 0.25\end{array}$ & $\begin{array}{l}-0.02 \\
\pm 0.04\end{array}$ & $\begin{array}{l}-0.02 \\
\pm 0.07\end{array}$ & 1.41 & 0.32 & 1.09 & Т 91 & 1 & 1.00 \\
\hline Tif1 & P10081 & & & & & $\begin{array}{r}-0.25 \\
\pm 0.06\end{array}$ & $\begin{array}{c}0.26 \\
\pm 0.16\end{array}$ & $\begin{array}{c}-0.01 \\
\pm 0.02\end{array}$ & $\begin{array}{c}0.03 \\
\pm 0.02\end{array}$ & 0.24 & 0.24 & -0.48 & 2 & 1 & 1.00 \\
\hline $\begin{array}{l}\text { Bmh1; } \\
\text { Bmh2 }\end{array}$ & $\begin{array}{l}\text { P29311; } \\
\text { P34730 }\end{array}$ & & & & & $\begin{array}{r}-0.20 \\
\pm 0.02 \\
\end{array}$ & $\begin{array}{c}0.21 \\
\pm 0.06 \\
\end{array}$ & $\begin{array}{c}0.12 \\
\pm 0.05 \\
\end{array}$ & $\begin{array}{c}0.01 \\
\pm 0.10 \\
\end{array}$ & -0.32 & 0.20 & -0.52 & S 104 & 1 & 0.95 \\
\hline
\end{tabular}


Supplementary Table 12. Cellular processes affected by Asc1p-dependent phosphorylation.

Proteins containing Asc1p-sensitive phospho-sites were assigned to different cellular processes. Protein names highlighted in green indicate that the proteins contain phospho-sites that are upregulated in the Asc1p mutant strains, accordingly, red indicates a down-regulation.

\begin{tabular}{|c|c|c|c|c|c|c|c|}
\hline 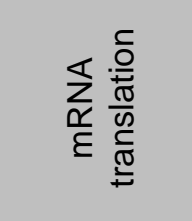 & 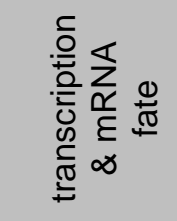 & 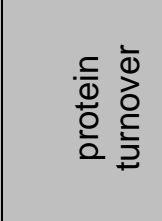 & 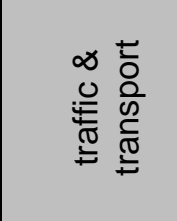 & 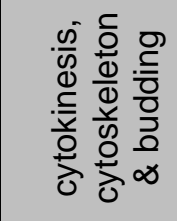 & 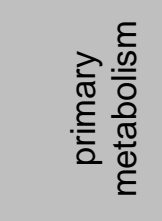 & 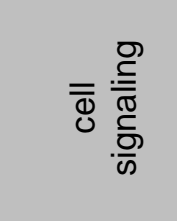 & 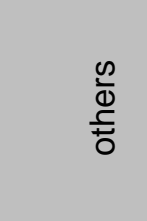 \\
\hline $\begin{array}{l}\text { Bud21 } \\
\text { Erb1 } \\
\text { Gcd6 } \\
\text { Ils1 } \\
\text { Kap123 } \\
\text { Kri1 } \\
\text { Mak5 } \\
\text { Mkt1 } \\
\text { Nop12 } \\
\text { Rlp7 } \\
\text { Rpg1 } \\
\text { Rpl12a/b } \\
\text { Rpl13a/b } \\
\text { Rps0a/b } \\
\text { Rrp36 } \\
\text { Sbp1** } \\
\text { Srp14 } \\
\text { Ssz1 } \\
\text { Sup35 } \\
\text { Tan1 } \\
\text { Tif35 } \\
\text { Tif5 } \\
\text { Yef3 } \\
\text { Tif1 } \\
\text { Prt1 } \\
\text { Cdc60 } \\
\text { Egd1 } \\
\text { Ett1 } \\
\text { Ncl1 } \\
\text { Pbp1 } \\
\text { Puf3 } \\
\text { Rpl7a/b } \\
\text { Rps19a/b } \\
\text { Rps1a/b* } \\
\text { Rps7b } \\
\text { Ssd1 } \\
\text { Sui3 } \\
\text { Tif4632 } \\
\text { Trm2 } \\
\text { Tsa1 } \\
\text { Vas1 } \\
\text { Yhr020w }\end{array}$ & $\begin{array}{l}\text { Air2 } \\
\text { Dig2 } \\
\text { Edc1 } \\
\text { Fip1 } \\
\text { Isw1 } \\
\text { Kin28 } \\
\text { Leo1 } \\
\text { Not3 } \\
\text { Rpo21 } \\
\text { Ski2 } \\
\text { Spn1 } \\
\text { Stb1 } \\
\text { Sub2 } \\
\text { Chd1 } \\
\text { Ctr9 } \\
\text { Fhl1 } \\
\text { Gat1 } \\
\text { Nrg2 } \\
\text { Pdr1 } \\
\text { Puf3 } \\
\text { Reb1 } \\
\text { Rsc2 } \\
\text { Ssn2 } \\
\text { Sum1 } \\
\text { Taf3 } \\
\text { Ume6 } \\
\text { Yap1 }\end{array}$ & $\begin{array}{l}\text { Cdc37 } \\
\text { Dcn1 } \\
\text { Pre8 } \\
\text { Rpn1 } \\
\text { Rpn7 } \\
\text { Shp1 } \\
\text { Vid27 } \\
\text { Bre5 } \\
\text { Cue4 } \\
\text { Def1 } \\
\text { Hsp42 } \\
\text { Pup2 } \\
\text { Rpn13 } \\
\text { Saf1** }\end{array}$ & $\begin{array}{l}\text { Akr1 } \\
\text { Apl5 } \\
\text { Bcp1 } \\
\text { Ent1 } \\
\text { Hrb1 } \\
\text { Imh11 } \\
\text { Kap123 } \\
\text { Kha1 } \\
\text { Myo3/5 } \\
\text { Pdr5 } \\
\text { Sec16 } \\
\text { Sec3 } \\
\text { Sec7 } \\
\text { She3 } \\
\text { Sla2 } \\
\text { Ysc84 } \\
\text { Ent2 } \\
\text { Gea2 } \\
\text { Vps13} \\
\text { Vtc2 } \\
\text { Bmh1/2** } \\
\text { Bre5 } \\
\text { Ecm21 } \\
\text { Ent3* } \\
\text { Esc1 } \\
\text { Gcs1 } \\
\text { Get2 } \\
\text { Gvp36 } \\
\text { Hxt1/3 } \\
\text { Kin2 } \\
\text { Mep2 } \\
\text { Mlp1 } \\
\text { Mnr2 } \\
\text { Sec21 } \\
\text { Sec31* } \\
\text { Tpo1 } \\
\text { Vps1 } \\
\text { Vtc3 }\end{array}$ & $\begin{array}{l}\text { Acm1 } \\
\text { Aim3 } \\
\text { Cmd1 } \\
\text { Ent1 } \\
\text { Myo3/5 } \\
\text { Pin4 } \\
\text { Rho4 } \\
\text { Sla2 } \\
\text { Smi1 } \\
\text { Ste20 } \\
\text { Ysc84 } \\
\text { Ent2 } \\
\text { Gea2 } \\
\text { Spa2 } \\
\text { Abp1 } \\
\text { Bbp1 } \\
\text { Bni1 } \\
\text { Cdc3 } \\
\text { Cdc28 } \\
\text { Ent3* } \\
\text { Gcs1 } \\
\text { Gvp36 } \\
\text { Hsp42 } \\
\text { Pfy1 } \\
\text { Spc110 } \\
\text { Ssd1 } \\
\text { Vip1 } \\
\text { Vps1 }\end{array}$ & $\begin{array}{l}\text { Csr1 } \\
\text { Gde1 } \\
\text { Gly1 } \\
\text { Leu1 } \\
\text { Nth1** } \\
\text { Pda1 } \\
\text { Pdc1 } \\
\text { Pdr16 } \\
\text { Pgm3 } \\
\text { Ser1 } \\
\text { Shm2 } \\
\text { Tpi1 } \\
\text { Acc1º } \\
\text { Met6 } \\
\text { Apa1 } \\
\text { Aro4 } \\
\text { Aro8 } \\
\text { Cho1 } \\
\text { Cys3 } \\
\text { Eno1/2 } \\
\text { Elo2 } \\
\text { Gpd2 } \\
\text { Gpm1 } \\
\text { Hom3 } \\
\text { Hom6 } \\
\text { Met17** } \\
\text { Met5 } \\
\text { Pct1 } \\
\text { Ser33 } \\
\text { Tdh1/2/3 } \\
\text { Thr4 } \\
\text { Ugp1 } \\
\text { Ura2 }\end{array}$ & $\begin{array}{l}\text { Akr1 } \\
\text { Cdc37 } \\
\text { Cmd1 } \\
\text { Dbf2 } \\
\text { Dig2 } \\
\text { Isc1 } \\
\text { Kin28 } \\
\text { Kin82 } \\
\text { Nnk1 } \\
\text { Nte1 } \\
\text { Pho13 } \\
\text { Pik1 } \\
\text { Ppq1 } \\
\text { Rcn1 } \\
\text { Rcn2 } \\
\text { Rho4 } \\
\text { Sec3 } \\
\text { Shp1 } \\
\text { Ste20 } \\
\text { Yak1 } \\
\text { Ypi1 } \\
\text { Spa2 } \\
\text { Bcy1 } \\
\text { Bmh1/2** } \\
\text { Bni1 } \\
\text { Cdc28 } \\
\text { Dbf20 } \\
\text { Glc8 } \\
\text { Kin2 } \\
\text { Kns1 } \\
\text { Meh1 } \\
\text { Orm2 } \\
\text { Pkh3 } \\
\text { Prr1 } \\
\text { Psk1 } \\
\text { Rad9 } \\
\text { Rho5 } \\
\text { Sch9 } \\
\text { Sfk1 } \\
\text { Vip1 } \\
\text { Yel043w } \\
\text { Ypr091c } \\
\text { Zeo1 }\end{array}$ & $\begin{array}{l}\text { Crp1 } \\
\text { Fyv8 } \\
\text { Guk1 } \\
\text { Iml2 } \\
\text { Pol1 } \\
\text { Rfa2 } \\
\text { Smc4 } \\
\text { Sng1 } \\
\text { Ty2a } \\
\text { Grx2 } \\
\text { Mdv1 } \\
\text { Par32 } \\
\text { Qri1 } \\
\text { Rad16 } \\
\text { Sod1 } \\
\text { Ty1b }++ \\
\text { Ycr023c } \\
\text { Yjl070c }\end{array}$ \\
\hline
\end{tabular}

also contains an up-regulated Asc1p-sensitive phospho-site

* regulation also found in either the asc $1^{\mathrm{T} 143 \mathrm{~A}}$ or the asc $1^{\mathrm{T} 143 \mathrm{E}}$ mutant strain

** regulation exclusively found in either the $\operatorname{asc} 1^{\top 143 \mathrm{~A}}$ or the $\operatorname{asc} 1^{\mathrm{T} 143 \mathrm{E}}$ mutant strain

$\downarrow$ regulated in the opposite direction in the asc $1^{\top 143 \mathrm{E}}$ strain compared to the asc $1^{\text {s }}$ strain

+ Ty2a-Dr3;Ty2a-C;Ty2a-Or1;Ty2a-Lr2;Ty2a-Gr2;Ty2a-F

++ Ty1b-Lr4;Ty1b-Pr1;Ty1b-Gr2;Ty1b-Pr2;Ty1b-Er1;Ty1b-Ml2;Ty1b-OI;Ty1b-Jr1;Ty1b-A;Ty1b-I1;Ty1b-

PI;Ty1b-Lr2 
Supplementary Table 13. Overview of data evaluation of SILAC-based Asc1p-Strep and Asc1DEp-Strep enrichment experiments with Perseus.

\begin{tabular}{|c|c|c|c|}
\hline No. & Command & \multicolumn{2}{|l|}{ Description } \\
\hline 1 & $\begin{array}{l}\text { Generic } \\
\text { matrix upload }\end{array}$ & \multicolumn{2}{|l|}{$\begin{array}{l}\text { proteinGroups.txt } \\
\text { normalized ratios etc. }\end{array}$} \\
\hline $\begin{array}{l}2.1 \\
2.2 \\
2.3\end{array}$ & $\begin{array}{l}\text { Filter rows } \\
\text { based on } \\
\text { categorical } \\
\text { column }\end{array}$ & \multicolumn{2}{|c|}{$\begin{array}{l}\text { Remove rows with }+ \text { in reverse column } \\
\text { Remove rows with }+ \text { in potential contaminant column } \\
\text { Remove rows with }+ \text { in only identified by site column }\end{array}$} \\
\hline 3 & Transform & \multicolumn{2}{|c|}{ Inverse ratios $(1 / x)$ when the control is not in the denominator } \\
\hline 4 & Transform & \multicolumn{2}{|l|}{$\log _{2}(x)$} \\
\hline 5 & Normalization & \multicolumn{2}{|l|}{ Subtract column median of ratios } \\
\hline 6 & $\begin{array}{l}\text { Combine } \\
\text { expression } \\
\text { columns }\end{array}$ & \multicolumn{2}{|c|}{$\begin{array}{l}\text { Eluate ratios minus respective proteome ratios } \\
\rightarrow \text { proteome-corrected eluate ratios }\end{array}$} \\
\hline $\begin{array}{l}7 \\
7.1 / 4 \\
7.2 / 5 \\
7.3 / 6 \\
7.7 \\
7.8\end{array}$ & $\begin{array}{l}\text { Categorical } \\
\text { annotation } \\
\text { rows }\end{array}$ & \multicolumn{2}{|c|}{$\begin{array}{l}\text { Group for Asc1p-Strep/control ratios and for Asc1DEp-Strep/control ratios: } \\
\text { - proteome-corrected eluate ratios } \\
\text { - eluate ratios } \\
\text { - proteome ratios } \\
\text { Group for two-sample } t \text {-test: } \\
\text { - eluate and proteome Asc1p-Strep/control ratios } \\
\text { - eluate and proteome Asc1DEp-Strep/control ratios }\end{array}$} \\
\hline $\begin{array}{l}8 \\
8.1 \\
8.2\end{array}$ & $\begin{array}{l}\text { Two-samples } \\
\text { test }\end{array}$ & \multicolumn{2}{|c|}{$\begin{array}{l}\text { Two-sample } t \text {-test }(p \text {-value }<0.05 \text { and } p \text {-value }<0.1) \\
\text { eluate versus proteome Asc1p-Strep/control ratios } \\
\text { eluate versus proteome Asc1DEp-Strep/control ratios }\end{array}$} \\
\hline $\begin{array}{l}9 \\
9.1 \\
9.2\end{array}$ & $\begin{array}{l}\text { One-sample } \\
\text { test }\end{array}$ & \multicolumn{2}{|c|}{$\begin{array}{l}\text { One-sample } t \text {-test }(p \text {-value }<0.05 \text { and } p \text {-value }<0.1) \\
\text { eluate ratios Asc1p-Strep } \\
\text { eluate ratios Asc1DEp-Strep }\end{array}$} \\
\hline & & $\begin{array}{l}\text { proteins with } 0-1 \text { valid proteome } \\
\text { ratio }\end{array}$ & $\begin{array}{l}\text { proteins with 2-3 valid proteome } \\
\text { ratios }\end{array}$ \\
\hline 10 & $\begin{array}{l}\text { Filter rows } \\
\text { based on } \\
\text { valid values }\end{array}$ & $\begin{array}{l}2 \text { proteome-corrected eluate ratios, } \\
\text { add categorical column }\end{array}$ & $\begin{array}{l}2 \text { proteome-corrected eluate ratios } \\
\geq 0.26\end{array}$ \\
\hline 11 & $\begin{array}{l}\text { Filter rows } \\
\text { based on } \\
\text { categorical } \\
\text { column }\end{array}$ & $\begin{array}{l}\text { Remove rows with keep from } \\
\text { previous step }\end{array}$ & - \\
\hline 12 & $\begin{array}{l}\text { Filter rows } \\
\text { based on } \\
\text { valid values }\end{array}$ & \multicolumn{2}{|c|}{1 proteome-corrected eluate ratios $<0.26$, add categorical column } \\
\hline 13 & $\begin{array}{l}\text { Filter rows } \\
\text { based on } \\
\text { categorical } \\
\text { column }\end{array}$ & \multicolumn{2}{|c|}{ Remove rows with keep from previous step } \\
\hline 14 & $\begin{array}{l}\text { Filter rows } \\
\text { based on } \\
\text { valid values }\end{array}$ & 2 eluate ratios $\geq 0.26$ & \\
\hline 15 & $\begin{array}{l}\text { Filter rows } \\
\text { based on } \\
\text { valid values }\end{array}$ & $\begin{array}{l}1 \text { eluate ratio }<0.26 \\
\text { add categorical column }\end{array}$ & \\
\hline 16 & $\begin{array}{l}\text { Filter rows } \\
\text { based on } \\
\text { categorical } \\
\text { column }\end{array}$ & $\begin{array}{l}\text { Remove rows with keep from } \\
\text { previous step }\end{array}$ & \\
\hline 17 & $\begin{array}{l}\text { Select rows } \\
\text { manually and } \\
\text { remove } \\
\text { selected rows }\end{array}$ & \multicolumn{2}{|c|}{$\begin{array}{l}\text { Check remaining candidates manually. Example for removed candidate: } \\
\text { Two eluate ratios of a protein that could not be proteome-corrected were } \\
\geq 0.26 \text {. For the third replicate, the protein was not detected in the eluate } \\
\text { fraction but with a ratio } \geq 0.26 \text { in the proteome indicating unspecific } \\
\text { enrichment of the protein in the other samples. }\end{array}$} \\
\hline
\end{tabular}


Supplementary Table 14. Expanded view for the Asc1p-dependent changes in the translatome. For the transcripts of each of the six samples the normalized readcounts are provided. The mean of these readcounts was calculated as well as the $\log _{2}$ ratio of these values (ascl/ASCl). The logarithmized ratios are colored according to the scale below the table. (FDR $=$ false discovery rate)

\begin{tabular}{|c|c|c|c|c|c|c|c|c|c|}
\hline \multirow{2}{*}{ gene } & \multirow{2}{*}{$\begin{array}{l}\text { asc1-1 } \\
\text { ASC1 }\end{array}$} & \multicolumn{3}{|c|}{$\begin{array}{c}\text { normalized } \\
\text { readcounts asc1. }\end{array}$} & \multicolumn{3}{|c|}{$\begin{array}{c}\text { normalized } \\
\text { readcounts } A S C 1 \\
\end{array}$} & \multirow{2}{*}{ likelihood } & \multirow{2}{*}{ FDR } \\
\hline & & 1 & 2 & 3 & 1 & 2 & 3 & & \\
\hline $\cos 8$ & 2.68 & 48 & 40 & 47 & 9 & 8 & 4 & 0.9965 & 1.7E-03 \\
\hline$Z P S 1$ & 2.35 & 494 & 801 & 783 & 174 & 111 & 124 & 0.9973 & $1.3 \mathrm{E}-03$ \\
\hline PRM5 & 1.84 & 268 & 214 & 260 & 78 & 53 & 76 & 0.9981 & 7.7E-04 \\
\hline HBN1 & 1.69 & 290 & 294 & 302 & 74 & 101 & 99 & 0.9995 & 1.4E-04 \\
\hline GSY1 & 1.65 & 286 & 267 & 310 & 98 & 99 & 78 & 0.9994 & 2.2E-04 \\
\hline HSP42 & 1.61 & 226 & 360 & 329 & 89 & 110 & 100 & 0.9916 & 3.5E-03 \\
\hline HSP26 & 1.48 & 67 & 59 & 78 & 31 & 22 & 20 & 0.9160 & $3.2 \mathrm{E}-02$ \\
\hline YCL021W-A & 1.45 & 138 & 181 & 189 & 62 & 57 & 67 & 0.9909 & $4.0 \mathrm{E}-03$ \\
\hline GAL3 & 1.38 & 110 & 140 & 167 & 54 & 60 & 46 & 0.9595 & $1.3 \mathrm{E}-02$ \\
\hline YLL053C & 1.36 & 494 & 411 & 543 & 212 & 201 & 153 & 0.9834 & 5.5E-03 \\
\hline ZRT1 & 1.35 & 4059 & 5193 & 5339 & 2244 & 1731 & 1744 & 0.9397 & $1.8 \mathrm{E}-02$ \\
\hline YKL070W & 1.34 & 78 & 110 & 124 & 40 & 41 & 42 & 0.9268 & $2.8 \mathrm{E}-02$ \\
\hline FET4 & 1.32 & 388 & 355 & 388 & 186 & 130 & 137 & 0.9935 & $2.6 \mathrm{E}-03$ \\
\hline$V T I 1$ & 1.27 & 275 & 181 & 236 & 110 & 87 & 89 & 0.9608 & $1.2 \mathrm{E}-02$ \\
\hline$A H A 1$ & 1.26 & 1459 & 1381 & 1438 & 678 & 556 & 548 & 0.9973 & $1.1 \mathrm{E}-03$ \\
\hline$A Q Y 2$ & 1.22 & 509 & 425 & 431 & 237 & 211 & 136 & 0.9322 & 2.4E-02 \\
\hline FKS3 & 1.22 & 134 & 150 & 162 & 63 & 67 & 62 & 0.9886 & $4.5 \mathrm{E}-03$ \\
\hline$S T I 1$ & 1.17 & 1427 & 1395 & 1514 & 646 & 608 & 670 & 0.9983 & $6.0 \mathrm{E}-04$ \\
\hline AGA1 & 1.16 & 209 & 294 & 208 & 94 & 107 & 117 & 0.9495 & $1.5 \mathrm{E}-02$ \\
\hline GAL80 & 1.15 & 444 & 382 & 586 & 211 & 215 & 209 & 0.9826 & $5.9 \mathrm{E}-03$ \\
\hline GSY2 & 1.12 & 428 & 427 & 450 & 205 & 189 & 206 & 0.9986 & 4.2E-04 \\
\hline NDJ1 & 1.07 & 160 & 213 & 251 & 95 & 95 & 107 & 0.9298 & $2.5 \mathrm{E}-02$ \\
\hline URA10 & 1.05 & 169 & 137 & 148 & 83 & 60 & 76 & 0.9182 & 3.1E-02 \\
\hline$H X K 1$ & 1.05 & 391 & 346 & 394 & 229 & 164 & 155 & 0.9409 & 1.7E-02 \\
\hline GRX3 & 1.02 & 847 & 882 & 856 & 385 & 416 & 477 & 0.9943 & $2.2 \mathrm{E}-03$ \\
\hline SMA2 & 1.01 & 228 & 297 & 285 & 126 & 122 & 153 & 0.9343 & 2.2E-02 \\
\hline DCS1 & 1.01 & 254 & 238 & 242 & 127 & 123 & 115 & 0.9921 & 3.3E-03 \\
\hline YGR161W-C & 1.00 & 335 & 337 & 341 & 169 & 172 & 167 & 0.9970 & $1.5 \mathrm{E}-03$ \\
\hline YHB1 & 0.95 & 9465 & 9433 & 8816 & 5197 & 4475 & 4671 & 0.9749 & 8.0E-03 \\
\hline$U B C 1$ & 0.91 & 674 & 818 & 776 & 356 & 416 & 436 & 0.9280 & 2.6E-02 \\
\hline LST8 & 0.91 & 429 & 415 & 525 & 267 & 221 & 243 & 0.9278 & $2.7 \mathrm{E}-02$ \\
\hline ALG13 & 0.90 & 281 & 280 & 285 & 149 & 162 & 142 & 0.9891 & $4.2 \mathrm{E}-03$ \\
\hline HSP78 & 0.86 & 462 & 415 & 420 & 266 & 215 & 234 & 0.9564 & 1.4E-02 \\
\hline APE1 & 0.84 & 435 & 401 & 409 & 237 & 203 & 256 & 0.9644 & $1.2 \mathrm{E}-02$ \\
\hline YMR315W & 0.84 & 942 & 820 & 931 & 508 & 451 & 548 & 0.9340 & 2.3E-02 \\
\hline FMP41 & 0.82 & 507 & 471 & 478 & 286 & 309 & 227 & 0.9207 & $2.8 \mathrm{E}-02$ \\
\hline GLO2 & 0.80 & 578 & 444 & 527 & 292 & 309 & 286 & 0.9352 & $2.2 \mathrm{E}-02$ \\
\hline GLK1 & 0.78 & 2089 & 2031 & 2049 & 1308 & 1137 & 1145 & 0.9392 & $1.9 \mathrm{E}-02$ \\
\hline TWF1 & 0.77 & 356 & 400 & 412 & 232 & 206 & 246 & 0.9140 & 3.2E-02 \\
\hline
\end{tabular}


Supplementary Table 14. Continued.

\begin{tabular}{|c|c|c|c|c|c|c|c|c|c|}
\hline \multirow{2}{*}{ gene } & \multirow{2}{*}{$\begin{array}{l}\text { asc1-1 } \\
\text { ASC1 }\end{array}$} & \multicolumn{3}{|c|}{$\begin{array}{c}\text { normalized } \\
\text { readcounts asc1- }\end{array}$} & \multicolumn{3}{|c|}{$\begin{array}{c}\text { normalized } \\
\text { readcounts } A S C 1\end{array}$} & \multirow{2}{*}{ likelihood } & \multirow{2}{*}{ FDR } \\
\hline & & 1 & 2 & 3 & 1 & 2 & 3 & & \\
\hline$M X R 1$ & 0.76 & 719 & 627 & 730 & 427 & 367 & 428 & 0.9046 & $3.4 \mathrm{E}-02$ \\
\hline SFG1 & 0.76 & 446 & 449 & 448 & 290 & 214 & 287 & 0.9011 & $3.5 \mathrm{E}-02$ \\
\hline ARC18 & 0.76 & 335 & 367 & 367 & 208 & 185 & 238 & 0.9075 & 3.3E-02 \\
\hline YKL151C & 0.76 & 522 & 478 & 541 & 314 & 291 & 306 & 0.9710 & $8.6 \mathrm{E}-03$ \\
\hline EXG2 & 0.71 & 386 & 371 & 378 & 232 & 252 & 208 & 0.9366 & $2.0 \mathrm{E}-02$ \\
\hline YDR327W & 0.70 & 1080 & 1115 & 1213 & 669 & 723 & 710 & 0.9187 & 3.0E-02 \\
\hline DFR1 & -0.66 & 249 & 275 & 279 & 418 & 412 & 440 & 0.9357 & $2.1 \mathrm{E}-02$ \\
\hline HTB1 & -0.68 & 604 & 705 & 684 & 1062 & 1057 & 1071 & 0.9396 & $1.8 \mathrm{E}-02$ \\
\hline FAA2 & -0.75 & 116 & 136 & 136 & 214 & 224 & 215 & 0.9290 & $2.5 \mathrm{E}-02$ \\
\hline $\mathrm{HO}$ & -0.75 & 443 & 449 & 487 & 763 & 745 & 813 & 0.9699 & $1.0 \mathrm{E}-02$ \\
\hline TDA1 & -0.91 & 112 & 122 & 109 & 199 & 214 & 230 & 0.9708 & $9.1 \mathrm{E}-03$ \\
\hline YHR177W & -0.93 & 71 & 63 & 81 & 125 & 142 & 144 & 0.9188 & 2.9E-02 \\
\hline URA4 & -1.02 & 625 & 609 & 658 & 1173 & 1321 & 1351 & 0.9922 & $3.1 \mathrm{E}-03$ \\
\hline AAC3 & -1.02 & 82 & 66 & 87 & 164 & 167 & 147 & 0.9702 & 9.7E-03 \\
\hline SNO1 & -1.04 & 161 & 114 & 139 & 298 & 272 & 283 & 0.9755 & $7.5 \mathrm{E}-03$ \\
\hline ARN1 & -1.06 & 267 & 278 & 246 & 674 & 495 & 482 & 0.9594 & 1.4E-02 \\
\hline ALD6 & -1.06 & 1507 & 1567 & 1758 & 3052 & 3510 & 3527 & 0.9682 & $1.1 \mathrm{E}-02$ \\
\hline YJL213W & -1.16 & 137 & 132 & 139 & 368 & 272 & 271 & 0.9800 & 7.0E-03 \\
\hline BAP3 & -1.23 & 51 & 43 & 53 & 121 & 119 & 105 & 0.9802 & $6.7 \mathrm{E}-03$ \\
\hline YIL 165C & -1.25 & 101 & 98 & 118 & 249 & 268 & 236 & 0.9970 & $1.4 \mathrm{E}-03$ \\
\hline$A Q R 1$ & -1.29 & 466 & 411 & 395 & 1232 & 968 & 904 & 0.9823 & $6.2 \mathrm{E}-03$ \\
\hline ECM13 & -1.36 & 126 & 121 & 125 & 367 & 308 & 277 & 0.9960 & $2.0 \mathrm{E}-03$ \\
\hline SNZ1 & -1.47 & 987 & 698 & 1224 & 2697 & 2602 & 2733 & 0.9933 & $2.8 \mathrm{E}-03$ \\
\hline RIB4 & -1.49 & 616 & 620 & 636 & 1362 & 1894 & 2011 & 0.9976 & 9.5E-04 \\
\hline TPO1 & -1.64 & 307 & 403 & 370 & 971 & 1297 & 1091 & 0.9961 & $1.8 \mathrm{E}-03$ \\
\hline YGR035C & -1.64 & 95 & 71 & 93 & 334 & 246 & 228 & 0.9909 & 3.7E-03 \\
\hline$Y M R 141 W-A$ & -1.67 & 18 & 18 & 10 & 49 & 48 & 49 & 0.9491 & 1.6E-02 \\
\hline FMP48 & -1.71 & 20 & 19 & 19 & 63 & 60 & 67 & 0.9877 & $4.8 \mathrm{E}-03$ \\
\hline TPO4 & -1.74 & 715 & 1388 & 1142 & 3217 & 4068 & 3577 & 0.9650 & 1.1E-02 \\
\hline TIS11 & -1.86 & 39 & 33 & 38 & 182 & 102 & 115 & 0.9857 & $5.1 \mathrm{E}-03$ \\
\hline URA3 & -1.87 & 440 & 341 & 391 & 1855 & 1003 & 1421 & 0.9936 & $2.4 \mathrm{E}-03$ \\
\hline URA1 & -2.14 & 833 & 1025 & 993 & 4083 & 4112 & 4328 & 1.0000 & $2.5 \mathrm{E}-05$ \\
\hline EEB1 & -2.56 & 245 & 264 & 238 & 1717 & 1361 & 1329 & 1.0000 & 8.6E-06 \\
\hline$M D H 2$ & -2.64 & 69 & 103 & 102 & 543 & 556 & 605 & 1.0000 & $2.0 \mathrm{E}-05$ \\
\hline
\end{tabular}

$\begin{array}{lllllllllll}\leq-2.5 & -2.0 & -1.5 & -1.0 & -0.5 & 0 & 0.5 & 1.0 & 1.5 & 2.0 & \geq 2.5\end{array}$ 
Supplementary Table 15. Mitochondrial proteins down-regulated in their abundance in the asc $1^{-}$ strain.

\begin{tabular}{|c|c|c|c|}
\hline proteins & protein IDs & description & $\begin{array}{l}\text { median } \log _{2} \\
\text { asc1-/ASC1 } \\
\text { ratio }\end{array}$ \\
\hline \multicolumn{4}{|c|}{ mitochondrial translation } \\
\hline $\operatorname{lmg} 1$ & P25626 & 54S ribosomal protein IMG1. mitochondrial & -0.50 \\
\hline Img2 & P25642 & 54S ribosomal protein IMG2. mitochondrial & -0.53 \\
\hline Mam33 & P40513 & Mitochondrial acidic protein MAM33 & -0.61 \\
\hline Mnp1 & P53163 & $54 S$ ribosomal protein L12. mitochondrial & -0.57 \\
\hline Mrp1 & P10662 & 37S ribosomal protein MRP1. mitochondrial & -0.71 \\
\hline Mrp13 & P12686 & 37S ribosomal protein MRP13. mitochondrial & -0.63 \\
\hline Mrpl15 & P36523 & 54 S ribosomal protein L15. mitochondrial & -0.48 \\
\hline Mrpl19 & P53875 & 54 S ribosomal protein L19. mitochondrial & -0.52 \\
\hline Mrpl3 & P36516 & 54S ribosomal protein L3. mitochondrial & -0.54 \\
\hline Mrpl33 & P20084 & 54S ribosomal protein L33. mitochondrial & -0.48 \\
\hline Mrpl38 & P35996 & 54 S ribosomal protein L38. mitochondrial & -0.30 \\
\hline Mrpl49 & P40858 & 54 S ribosomal protein L49. mitochondrial & -0.55 \\
\hline Mrpl51 & Q06090 & $54 S$ ribosomal protein L51. mitochondrial & -0.53 \\
\hline Mrps8 & Q03799 & 37S ribosomal protein S8. mitochondrial & -0.38 \\
\hline Mss51 & P32335 & Protein MSS51. mitochondrial & -0.46 \\
\hline Rsm10 & Q03201 & 37S ribosomal protein S10. mitochondrial & -0.49 \\
\hline Rsm7 & P47150 & 37S ribosomal protein S7. mitochondrial & -0.52 \\
\hline Tuf1 & P02992 & Elongation factor Tu. mitochondrial & -0.62 \\
\hline Var1 & P02381 & Ribosomal protein VAR1. mitochondrial & -0.87 \\
\hline \multicolumn{4}{|c|}{ mitochondrial protein import } \\
\hline Mia40 & P36046 & $\begin{array}{l}\text { Mitochondrial intermembrane space import and } \\
\text { assembly protein } 40\end{array}$ & -0.62 \\
\hline Tim10 & P87108 & $\begin{array}{l}\text { Mitochondrial import inner membrane translocase } \\
\text { subunit TIM10 }\end{array}$ & -0.68 \\
\hline Tim12 & P32830 & $\begin{array}{l}\text { Mitochondrial import inner membrane translocase } \\
\text { subunit TIM12 }\end{array}$ & -0.31 \\
\hline Tim44 & Q01852 & $\begin{array}{l}\text { Mitochondrial import inner membrane translocase } \\
\text { subunit TIM44 }\end{array}$ & -0.28 \\
\hline Tim50 & Q02776 & $\begin{array}{l}\text { Mitochondrial import inner membrane translocase } \\
\text { subunit TIM50 }\end{array}$ & -0.28 \\
\hline Tim8 & P57744 & $\begin{array}{l}\text { Mitochondrial import inner membrane translocase } \\
\text { subunit TIM8 }\end{array}$ & -0.30 \\
\hline Tim9 & 074700 & $\begin{array}{l}\text { Mitochondrial import inner membrane translocase } \\
\text { subunit TIM9 }\end{array}$ & -0.66 \\
\hline Tom20 & P35180 & Mitochondrial import receptor subunit TOM20 & -0.26 \\
\hline Tom22 & P49334 & Mitochondrial import receptor subunit TOM22 & -0.42 \\
\hline Tom40 & P23644 & Mitochondrial import receptor subunit TOM40 & -0.47 \\
\hline Tom7 & P53507 & Mitochondrial import receptor subunit TOM7 & -0.42 \\
\hline Tom70 & P07213 & Mitochondrial import receptor subunit TOM70 & -0.58 \\
\hline
\end{tabular}


Supplementary Table 15 continued 1.

\begin{tabular}{|c|c|c|c|}
\hline proteins & protein IDs & description & $\begin{array}{l}\text { median } \log _{2} \\
\text { asc1-/ASC1 } \\
\text { ratio }\end{array}$ \\
\hline \multicolumn{4}{|c|}{ further mitochondrial transport processes } \\
\hline Aac1 & P04710 & ADP, ATP carrier protein 1 & -0.42 \\
\hline Ctp1 & P38152 & Tricarboxylate transport protein & -0.97 \\
\hline Dic1 & Q06143 & Mitochondrial dicarboxylate transporter & -0.80 \\
\hline Ggc1 & P38988 & $\begin{array}{l}\text { Putative mitochondrial carrier protein } \\
\text { YHM1/SHM1 }\end{array}$ & -0.67 \\
\hline Mir1 & P23641 & Mitochondrial phosphate carrier protein & -0.57 \\
\hline Om14 & P38325 & Mitochondrial outer membrane protein OM14 & -0.45 \\
\hline Pet9 & P18239 & ADP, ATP carrier protein 2 & -0.40 \\
\hline Por1 & P04840 & Mitochondrial outer membrane protein porin 1 & -0.47 \\
\hline \multicolumn{4}{|c|}{ respiration } \\
\hline Atp1 & P07251 & ATP synthase subunit $\alpha$, mitochondrial & -0.52 \\
\hline Atp2 & P00830 & ATP synthase subunit $\beta$, mitochondrial & -0.49 \\
\hline Atp3 & P38077 & ATP synthase subunit $\gamma$, mitochondrial & -0.38 \\
\hline Atp4 & P05626 & ATP synthase subunit 4 , mitochondrial & -0.32 \\
\hline Atp5 & P09457 & ATP synthase subunit 5 , mitochondrial & -0.43 \\
\hline Atp7 & P30902 & ATP synthase subunit $d$, mitochondrial & -0.33 \\
\hline Atp15 & P21306 & ATP synthase subunit $\varepsilon$, mitochondrial & -0.41 \\
\hline Atp16 & Q12165 & ATP synthase subunit $\delta$, mitochondrial & -0.32 \\
\hline Atp17 & Q06405 & ATP synthase subunit $f$, mitochondrial & -0.28 \\
\hline Atp18 & P81450 & ATP synthase subunit $\mathrm{J}$, mitochondrial & -0.34 \\
\hline Cox4 & P04037 & Cytochrome c oxidase subunit 4 , mitochondrial & -0.39 \\
\hline Cox6 & P00427 & Cytochrome c oxidase subunit 6 , mitochondrial & -0.26 \\
\hline Nde1 & P40215;Q07500 & $\begin{array}{l}\text { External NADH-ubiquinone oxidoreductase 2, } \\
\text { mitochondrial }\end{array}$ & -0.42 \\
\hline Sdh1 & Q00711;P47052 & $\begin{array}{l}\text { Succinate dehydrogenase [ubiquinone] } \\
\text { flavoprotein subunit } 2 \text {, mitochondrial }\end{array}$ & -0.57 \\
\hline Sdh2 & P21801 & $\begin{array}{l}\text { Succinate dehydrogenase [ubiquinone] iron- } \\
\text { sulfur subunit, mitochondrial }\end{array}$ & -0.32 \\
\hline Tim11 & P81449 & ATP synthase subunit e, mitochondrial & -0.31 \\
\hline \multicolumn{4}{|c|}{ tricarboxylic acid cycle } \\
\hline Aco2 & P39533 & Probable aconitate hydratase 2 & -0.55 \\
\hline Kgd1 & P20967 & $\begin{array}{l}\text { 2-oxoglutarate dehydrogenase E1 component, } \\
\text { mitochondrial }\end{array}$ & -0.47 \\
\hline Lsc2 & P53312 & $\begin{array}{l}\text { Succinyl-CoA ligase [ADP-forming] subunit } \beta \text {, } \\
\text { mitochondrial }\end{array}$ & -0.30 \\
\hline \multicolumn{4}{|c|}{ assembly of respiratory chain complexes } \\
\hline Cbp3 & P21560 & Protein CBP3, mitochondrial & -0.28 \\
\hline Cbp4 & P37267 & Assembly factor CBP4 & -0.30 \\
\hline Coa1 & P40452 & Cytochrome oxidase assembly protein 1 & -0.29 \\
\hline Cox15 & P40086 & Cytochrome c oxidase assembly protein COX15 & -0.32 \\
\hline Yta12 & P40341 & $\begin{array}{l}\text { Mitochondrial respiratory chain complexes } \\
\text { assembly protein RCA1 }\end{array}$ & -0.39 \\
\hline
\end{tabular}


Supplementary Table 15 continued 2.

\begin{tabular}{|c|c|c|c|}
\hline proteins & protein IDs & description & $\begin{array}{l}\text { median } \log _{2} \\
\text { asc1-/ASC1 } \\
\text { ratio }\end{array}$ \\
\hline \multicolumn{4}{|c|}{ mitochondrial contact site and cristae organizing system (MICOS) complex } \\
\hline Mic10 & Q96VH5 & UPF0327 protein YCL057C-A & -0.31 \\
\hline Mic12 & P38341 & Uncharacterized protein YBR262C & -0.40 \\
\hline Mic19 & P43594 & UPF0726 protein YFR011C & -0.41 \\
\hline Mic60 & P36112 & Uncharacterized protein YKR016W & -0.31 \\
\hline \multicolumn{4}{|c|}{ glycine decarboxylase complex } \\
\hline Gcv1 & P48015 & Aminomethyltransferase, mitochondrial & -0.47 \\
\hline Gcv2 & P49095 & $\begin{array}{l}\text { Glycine dehydrogenase [decarboxylating], } \\
\text { mitochondrial }\end{array}$ & -0.75 \\
\hline Gcv3 & P39726 & Glycine cleavage system $\mathrm{H}$ protein, mitochondrial & -0.62 \\
\hline \multicolumn{4}{|c|}{ protein folding and degradation } \\
\hline Hsp10 & P38910 & $10 \mathrm{kDa}$ heat shock protein, mitochondrial & -0.40 \\
\hline Phb1 & P40961 & Prohibitin-1 & -0.27 \\
\hline Pim1 & P36775 & Lon protease homolog, mitochondrial & -0.59 \\
\hline \multicolumn{4}{|c|}{ proteins with described mitochondria-associated functions } \\
\hline Adh3 & P07246 & Alcohol dehydrogenase 3, mitochondrial & -0.38 \\
\hline Adk1 & P07170 & Adenylate kinase 1 & -0.70 \\
\hline Arg5,6 & Q01217 & Protein ARG5,6, mitochondrial & -0.34 \\
\hline Arg8 & P18544 & Acetylornithine aminotransferase, mitochondrial & -0.36 \\
\hline Arh1 & P48360 & $\begin{array}{l}\text { Probable NADPH:adrenodoxin oxidoreductase, } \\
\text { mitochondrial }\end{array}$ & -0.26 \\
\hline Dld1 & P32891 & $\begin{array}{l}\text { D-lactate dehydrogenase [cytochrome] } 1, \\
\text { mitochondrial }\end{array}$ & -0.49 \\
\hline Dld2 & P46681 & $\begin{array}{l}\text { D-lactate dehydrogenase [cytochrome] } 2 \text {, } \\
\text { mitochondrial }\end{array}$ & -0.26 \\
\hline Erv1 & P27882 & $\begin{array}{l}\text { Mitochondrial FAD-linked sulfhydryl oxidase } \\
\text { ERV1 }\end{array}$ & -0.54 \\
\hline Hem1 & P09950 & 5-aminolevulinate synthase, mitochondrial & -0.70 \\
\hline Isc1 & P40015 & Inositol phosphosphingolipids phospholipase C & -0.63 \\
\hline Mae1 & P36013 & NAD-dependent malic enzyme, mitochondrial & -0.48 \\
\hline Mmf1 & P40185 & Protein MMF1, mitochondrial & -0.38 \\
\hline Mss116 & P15424 & $\begin{array}{l}\text { ATP-dependent RNA helicase MSS116, } \\
\text { mitochondrial }\end{array}$ & -0.47 \\
\hline Num1 & Q00402 & Nuclear migration protein NUM1 & -0.30 \\
\hline Prx1 & P34227 & Mitochondrial peroxiredoxin PRX1 & -0.45 \\
\hline Sod2 & P00447 & Superoxide dismutase [Mn], mitochondrial & -0.52 \\
\hline Uth1 & P36135 & Protein UTH1 & -0.76 \\
\hline Yhm2 & Q04013 & Mitochondrial DNA replication protein YHM2 & -0.80 \\
\hline
\end{tabular}


Supplementary Table 15 continued 3.

\begin{tabular}{|l|l|l|c|}
\hline proteins & protein IDs & description & $\begin{array}{l}\text { median } \log _{2} \\
\text { asc1-/ASC1 } \\
\text { ratio }\end{array}$ \\
\hline \multicolumn{2}{|l|}{ further proteins found to be associated with mitochondria } & -0.54 \\
\hline Aim36 & Q03798 & Uncharacterized protein YMR157C & -0.39 \\
Aim9 & P40053 & Uncharacterized protein YER080W & -1.07 \\
Cpr1 & P14832 & Peptidyl-prolyl cis-trans isomerase & -0.33 \\
Gpm1 & P00950 & Phosphoglycerate mutase 1 & -0.33 \\
Imo32 & P53219 & Uncharacterized abhydrolase domain-containing & -1.44 \\
Msc1 & protein YGR031W & -0.54 \\
Ncp1 & P16603 & Meiotic sister chromatid recombination protein 1 & -0.49 \\
Pep4 & P07267 & NADP-cytochrome P450 reductase & -0.28 \\
Pth2 & P34222 & Saccharopepsin & -0.48 \\
Tdh3 & P00359 & Glyceraldehyde-3-phosphate dehydrogenase 3 & -0.37 \\
Tma19 & P35691 & Translationally-controlled tumor protein homolog & -0.28 \\
Tpi1 & P00942 & Triosephosphate isomerase & -28 \\
\hline
\end{tabular}

Supplementary Table 16. Proteasomal proteins up-regulated in their abundance in the asc1strain.

\begin{tabular}{|l|l|l|l|}
\hline proteins & protein IDs & description & $\begin{array}{l}\text { median log } \\
\text { asc1-/ASC1 } \\
\text { ratio }\end{array}$ \\
\hline 20S core particle & Proteasome subunit $\beta$ type-5 & 0.42 \\
\hline Pre2 & P30656 & Proteasome subunit $\beta$ type-1 & 0.29 \\
Pre3 & P38624 & Proteasome subunit $\beta$ type-7 & 0.34 \\
Pre4 & P30657 & Proteasome subunit $\alpha$ type-6 & 0.39 \\
Pre5 & P40302 & Proteasome subunit $\alpha$ type-4 & 0.32 \\
Pre6 & P40303 & Proteasome subunit $\beta$ type-6 & 0.48 \\
Pre7 & P23724 & Proteasome subunit $\alpha$ type-2 & 0.28 \\
Pre8 & P23639 & Proteasome subunit $\alpha$ type-3 & 0.30 \\
Pre9 & P23638 & Probable proteasome subunit $\alpha$ type-7 & 0.33 \\
Pre10 & P21242 & Proteasome subunit $\beta$ type-2 & 0.30 \\
Pup1 & P25043 & Proteasome subunit $\alpha$ type-5 & 0.31 \\
Pup2 & P2379 & Proteasome subunit $\beta$ type-3 & 0.33 \\
Pup3 & P25451 & Proteasome subunit $\alpha$ type-1 & 0.41 \\
Scl1 & P21243 & 26S proteasome regulatory subunit RPN13 & 0.28 \\
\hline 19S regulatory particle & 26S proteasome regulatory subunit RPN5 & 0.27 \\
\hline Rpn13 & O13563 & Q12250
\end{tabular}


Abbreviations

aa

$=$ amino acid

$\mathrm{AC}$

$=$ adenylyl cyclase

AMP

$=$ adenosine monophosphate

Aux

= auxotrophy

3-AT

= 3-amino-1,2,4-triazole

BCA

$=$ bicinchoninic acid

bp

$=$ base pair

BSA

= bovine serum albumin

cAMP

= cyclic adenosine monophosphate

cDNA

= complementary DNA

CID

$=$ collision-induced dissociation

C-terminal/-terminus $=$ carboxyl-terminus

d

$=$ days

DE

$=\mathrm{R} 38 \mathrm{D} \mathrm{K} 40 \mathrm{E}$

DETA-NONOate

$=(Z)-1-[N-(2-$ Aminoethyl $)-N-(2$-ammonioethyl $)$ amino $]$ diazen-1-ium

1,2-diolate)

DNA = deoxyribonucleic acid

dNTP = deoxynucleoside triphosphate

DTT = dithiotreitol

ECL = enhanced chemoluminescence

EDTA = ethylenediaminetetraacetic acid

EGTA = ethylene glycol tetraacetic acid

et al. $\quad=$ et alii

FASP = filter-aided sample preparation

FDR = false discovery rate

FT $\quad=$ fourier transform

$\mathrm{G}_{1} / \mathrm{G}_{2} \quad$ = gap phase $1 / 2$

GDP = guanosine diphosphate

glc $\quad=$ glucose

GPCR = G-protein-coupled receptor

GTP = guanosine triphosphate

$\mathrm{h}$

$=\operatorname{hour}(\mathrm{s})$ 


\begin{tabular}{|c|c|}
\hline $\mathrm{HCC}$ & $=$ hepatocellular carcinoma \\
\hline $\mathrm{HCD}$ & $=$ higher energy collisional dissociation \\
\hline HEK293 & $=$ human embryonic kidney 293 \\
\hline HeLa & $=$ Henrietta Lacks \\
\hline Huh7 & $=$ human hepatocellular carcinoma \\
\hline ID & $=$ identifier \\
\hline IRES & $=$ internal ribosomal entry site \\
\hline LB & $=$ lysogeny broth \\
\hline $\mathrm{LC}$ & $=$ liquid chromatography \\
\hline M & $=$ mitosis \\
\hline MAPK & $=$ mitogen activated protein kinase \\
\hline $\min$ & $=\operatorname{minute}(\mathrm{s})$ \\
\hline miRISC & $=$ miRNA-induced silencing complex \\
\hline miRNA & $=$ micro RNA \\
\hline mRNA & $=$ messenger RNA \\
\hline mRNP & $=$ ribonucleoprotein containing mRNA \\
\hline MS & $=$ mass spectrometry \\
\hline MSA & $=$ multistage activation \\
\hline MV & $=$ minimal with vitamins \\
\hline N/A & $=$ not applicable \\
\hline $\mathrm{NaN}$ & $=$ not $\mathrm{a}$ number \\
\hline N-terminal/-terminus & $=$ amino-terminus \\
\hline OD & $=$ optical density \\
\hline ORF & $=$ open reading frame \\
\hline PACAP & $=$ pituitary adenylyl cyclase-activating polypeptide \\
\hline PAGE & $=$ polyacrylamide gel electrophoresis \\
\hline P-bodies & $=$ processing bodies \\
\hline PBS & $=$ phosphate buffered saline \\
\hline PCR & $=$ polymerase chain reaction \\
\hline PEG & $=$ polyethylene glycol \\
\hline PKA & $=$ protein kinase $\mathrm{A}$ \\
\hline PKC & $=$ protein kinase $\mathrm{C}$ \\
\hline PMSF & $=$ phenylmethane sulfonyl fluoride \\
\hline PTM & $=$ post-translational modification \\
\hline
\end{tabular}




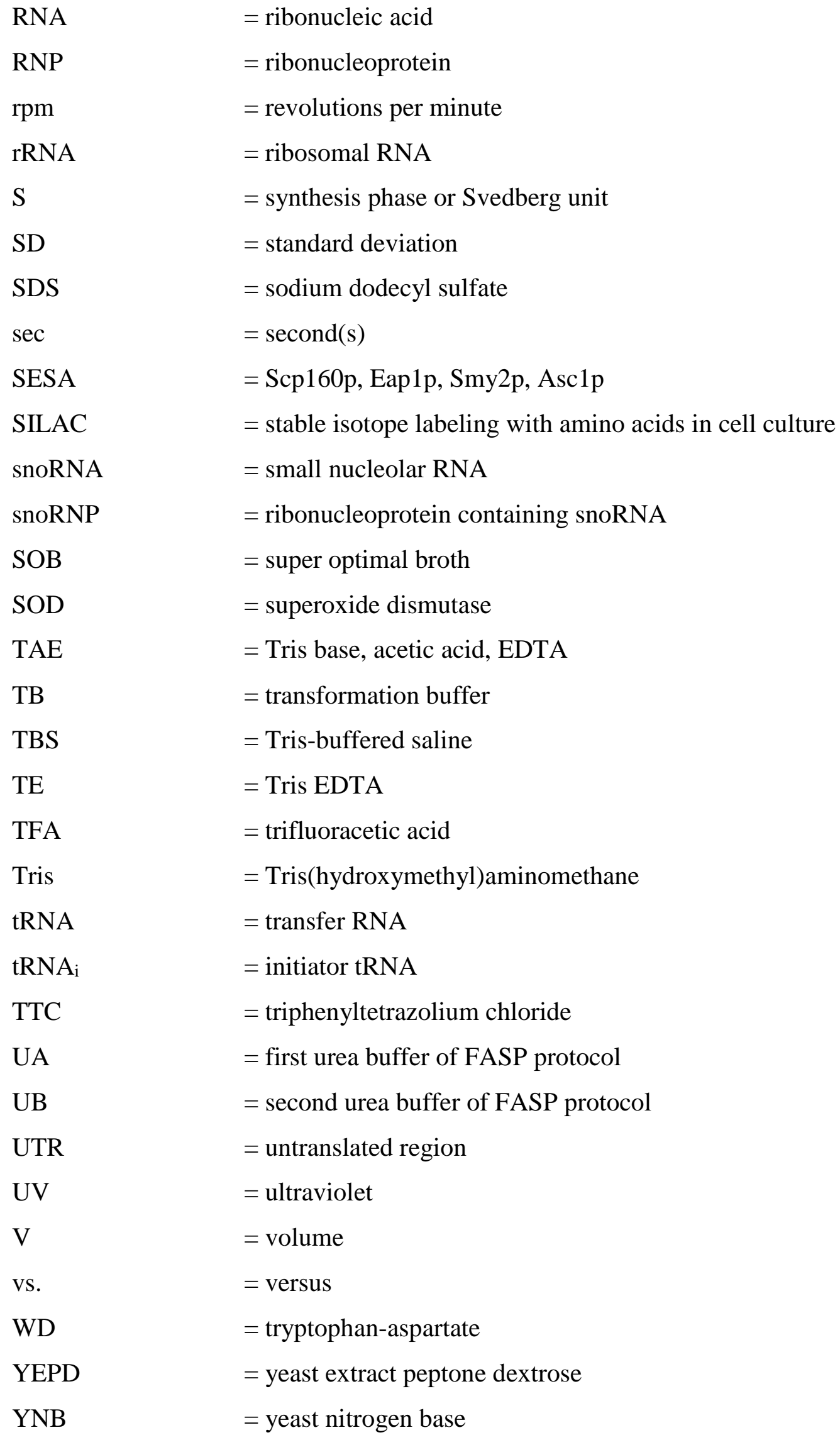

